\title{
Experiences of care continuity and recovery for people at the interface of primary and secondary mental health care in Wales: a thematic discourse analytic approach
}

\section{By}

Nicholas Weaver

Submitted to Swansea University in fulfilment of the requirements for the Degree of Doctor of Philosophy

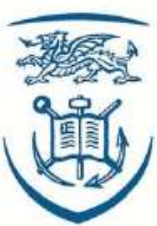

Swansea University

Prifysgol Abertawe 
This work has not previously been accepted in substance for any degree and is not being concurrently submitted in candidature for any degree.

(candidate)

(date)

This thesis is the result of my own investigations, except where otherwise stated; other sources are acknowledged by footnotes giving explicit references and a bibliography is appended.

(candidate)

(date)

I give consent for the thesis, if accepted, to be made available online in the University's Open Access Repository and for inter-library loan, and for the title and summary to be made available to outside organisations.

(candidate)

(date) 


\begin{abstract}
This study explores talk about experiences of care continuity and recovery for people with serious mental health issues at the interface of primary and secondary mental healthcare in Wales. The approach adopted was qualitative, employing a methodology of thematic discourse analysis of talk generated by in-depth, semi-structured interviews. The study was conducted in two phases, the first round involving $(n=10)$ service users who had transited from secondary to primary care, and the second round involving $(n=16)$ workers and practitioners involved in supporting service users at the primary-secondary interface.
\end{abstract}

The main findings were first that participants' expectations were misaligned with transforming service structures and interfaces. Second, participants constructed competing versions of recovery in their talk. Third, analysis of talk revealed experiences of care discontinuities in multiple areas, concentrated particularly at the primary level. On this basis, it is argued that a proliferation of competing recovery versions, and misaligned expectations of transforming services, are closely allied with escalating service system complexity and fragmentation, with detrimental implications for care continuity and coordination.

The role of neoliberalist, policy-based recovery versions such as the Mental Health (Wales) Measure 2010, is highlighted in colonising the recovery concept. The social theory of system versus lifeworld is used to provide a theoretical context for understanding recovery colonisation, potential distortion of the concept, and how this may be an additional factor for systemic complexity and fragmentation. In order to contain escalating service complexity, a balance needs to be struck between top-down, colonised implementations of recovery and bottom-up recovery versions rooted in the individual lived experience of the service user.

The notion of the public sphere is also employed as a basis for study implications, to articulate a context for ideal speech situations where there is free function of communicative action at the intersubjective level of the lifeworld. Peer support groups, recovery colleges and online forums may serve as instances of a public sphere within which fruitful opportunities for education and construction of recovery approaches may be reclaimed. User / survivor activism may also have a role in securing this vital context for recovery construction. 


\section{Acknowledgements}

The process of writing and completing this $\mathrm{PhD}$ thesis has been a significant undertaking and a landmark event in my own life. I first want to acknowledge that without the good work of so many providing support for those with mental health and related issues, I simply would not have had the opportunity to engage with the field which forms the context for this work. I also want to thank those who have tutored, lectured and encouraged me during the undergraduate course for mental health nursing at Swansea University, enabling me to enter the field. In particular I would like to thank Dr Julia Terry and Hywel Thomas, who later provided initial advice and contact points for me to commence my journey along the $\mathrm{PhD}$ path.

I am indebted to all of my participants who enthusiastically and reliably gave up their time to invest their own perspectives into this work. Without this, my research would have been nothing since there would have been no data on which it could be based!

Thank you so much to all three of my supervisors, Professor Michael Coffey, Dr Jeanette Hewitt and Professor Deb Fitzsimmons. You have guided me throughout various stages of the project, as I endeavoured to enter the field of academia, and research my particular focus on mental health issues and recovery. It has been a transformative journey and, as I have said before, I am now able to access learning that I would not have been able to previously. A particular thank you to my main supervisor, Michael, who never stopped believing in my potential to complete this and other projects, and has supported me throughout.

I am also indebted to the support that has been provided by my funders, RCBC Wales, especially in relation to the regular Community of Scholars events which were ideal for developing my potential as an advanced clinician. University support from the PGR administrators, Katie Retallick and Maria Davis has also been of the highest quality and has consistently been a great help.

A variety of supportive friends and counsellors should also be thanked without whom I would also not have been able to complete the long journey towards completion. These include Rhys, Mark, Fred, Joe and Ivan, but there are many others.

But my most heartfelt debt of gratitude is directed towards those closest to me. My wife Zoe's support was crucial so very often, particularly with respect to the two children we have had whilst doing this $\mathrm{PhD}$, Bridget and James, whose infantile support I would also like to acknowledge. From the start I think she has known the sacrifice she would need to make, and she has done so admirably. I would also like to thank God for restoring me to the point of this undertaking, sustaining me continually throughout the journey, and without whom I am nothing. Finally, my many thanks go to my Dad whose support is obvious and gratefully received, and of course my mother-in-law, Diana, who looked after both babies so many times in my stead, thereby allowing me to focus on my work! 


\section{Contents}

\section{Chapter 1: Introduction}

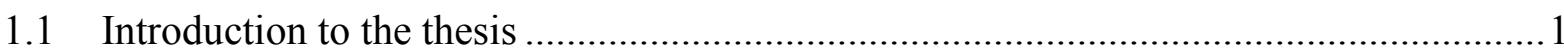

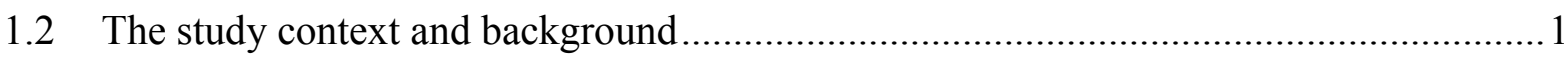

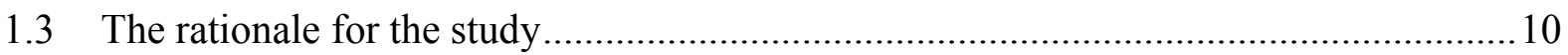

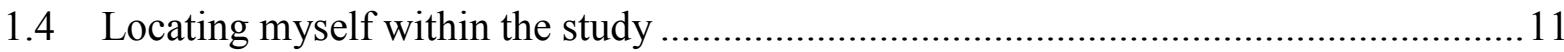

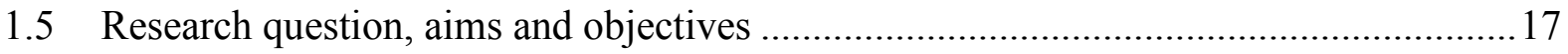

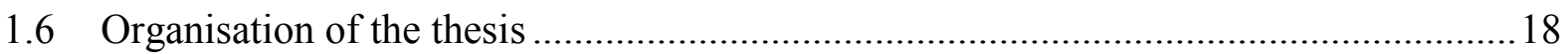

Chapter 2: Reviewing the evidence for care continuity and complexity in the provision of recovery-focused mental healthcare

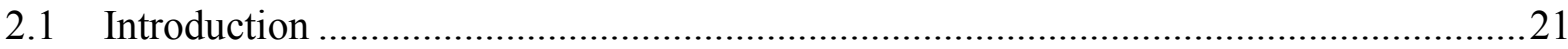

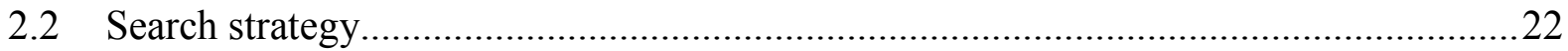

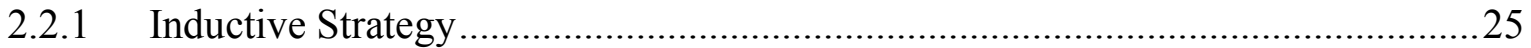

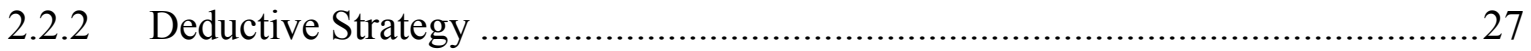

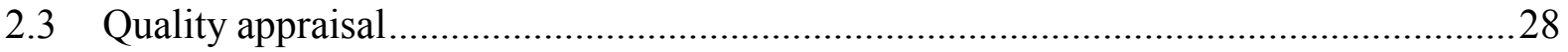

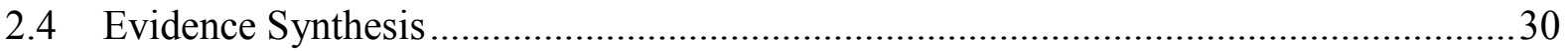

2.5 Complexity theory, mental health services and the impact of policy............................ 31

2.5.1 Complexity theory and mental healthcare systems ............................................ 31

2.5.2 The impact of policy on mental health systems complexity ................................. 34

2.6 Recovery as the focus of contemporary mental health policy..................................... 36

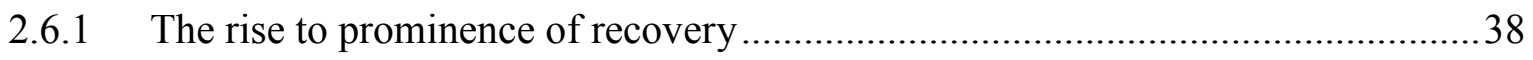

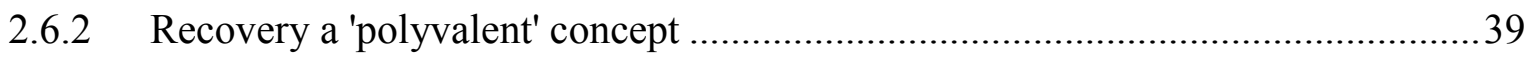

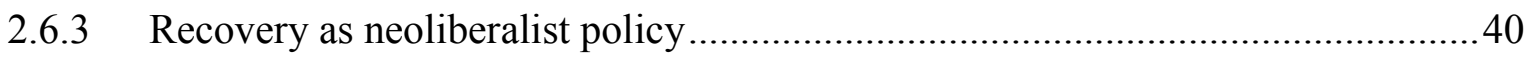

2.7 Care continuity as a multi-dimensional concept ..................................................... 42

2.7.1 Theoretical introduction to care continuity as a multi-dimensional concept......... 45

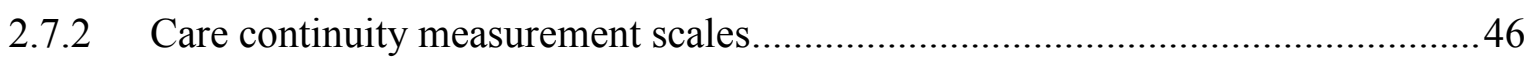

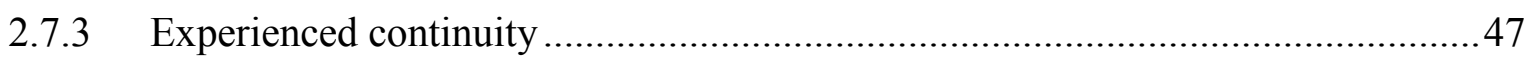

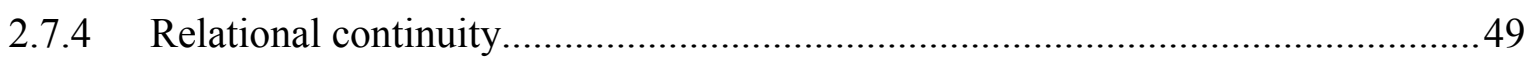

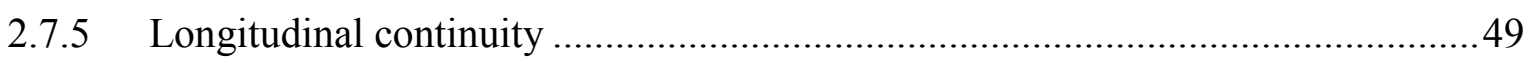




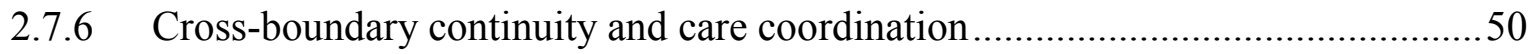

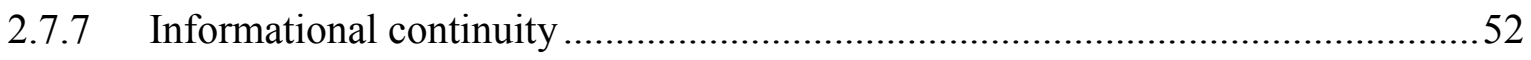

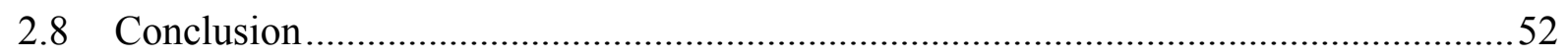

\section{Chapter 3: Methodology and methods}

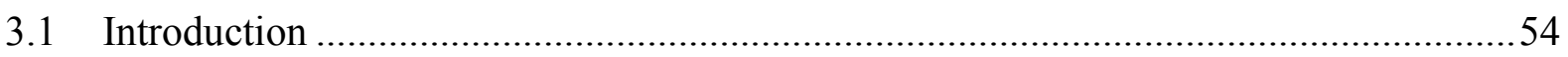

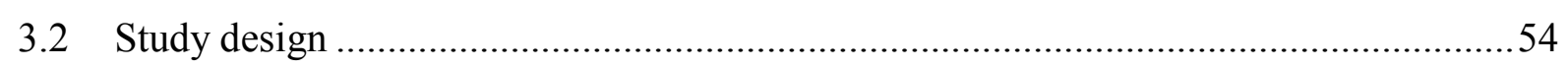

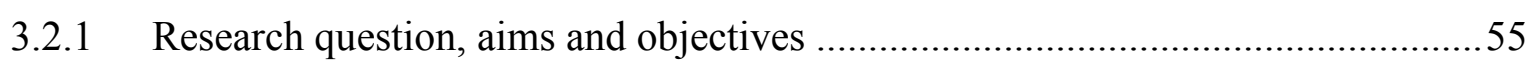

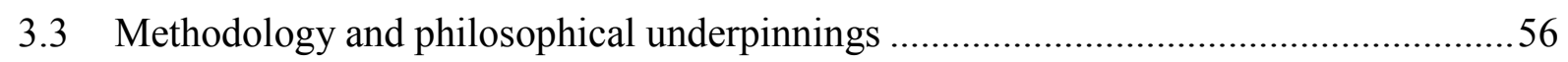

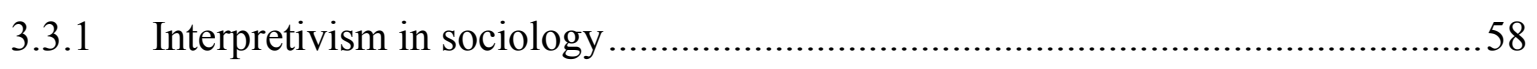

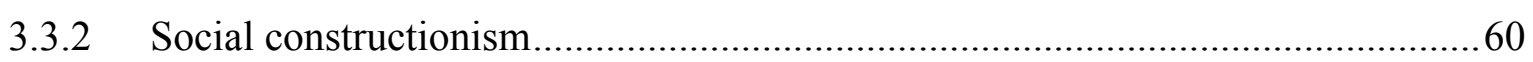

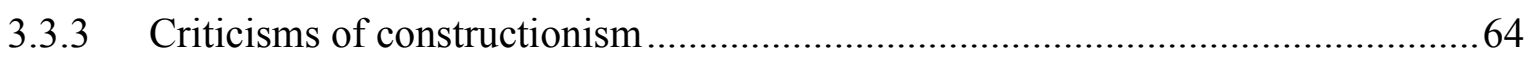

3.3.4 Constructionism and critical realism - a reconciliation and solution to the problem

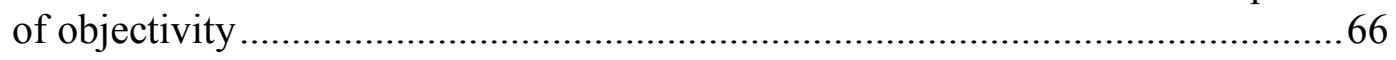

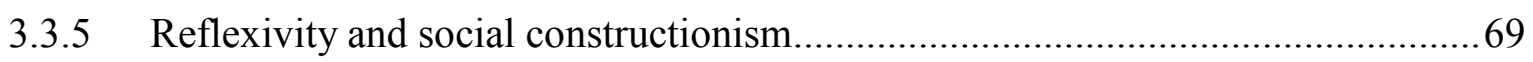

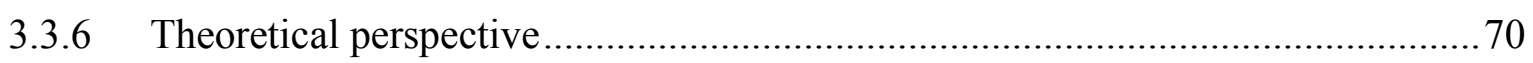

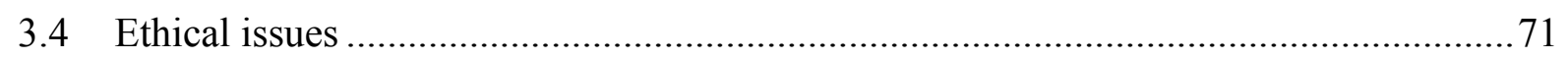

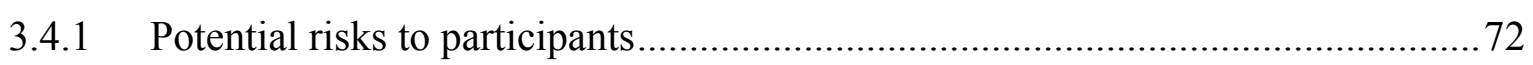

3.4.2 Ensuring confidentiality and anonymity........................................................ 72

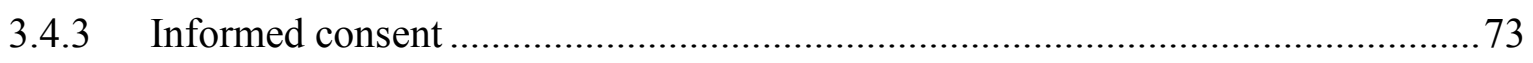

3.4.4 Briefing and debriefing of participants............................................................. 74

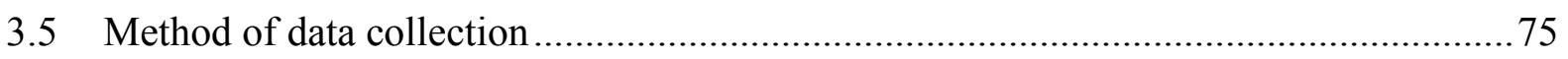

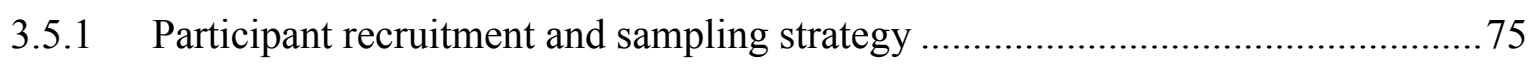

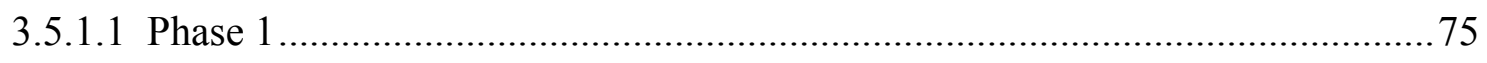

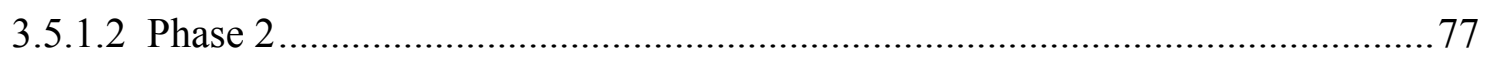

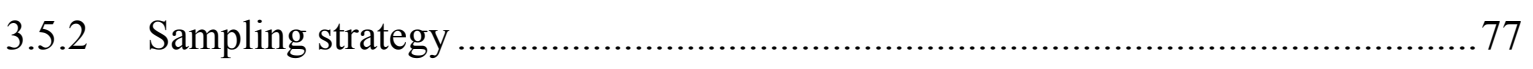

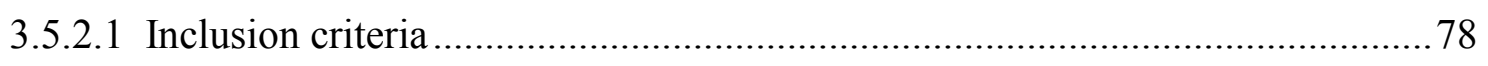

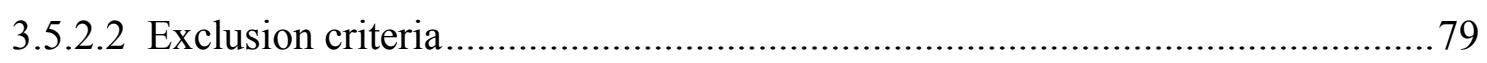

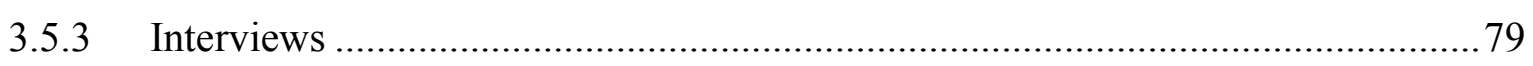

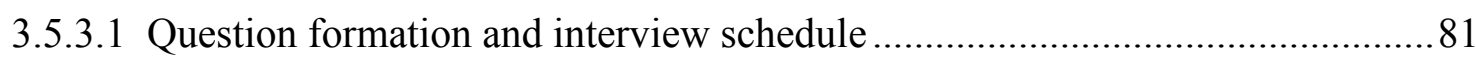

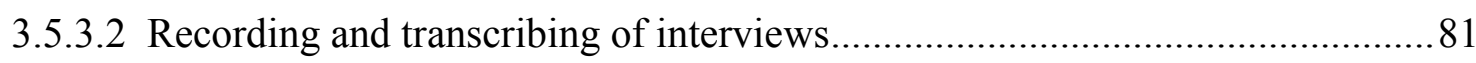

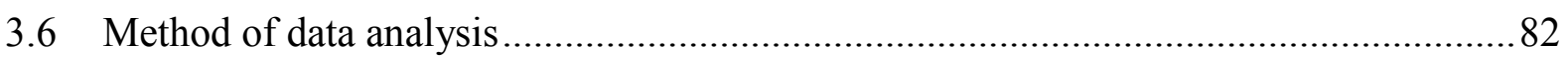




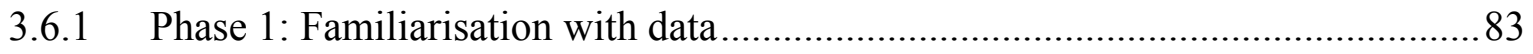

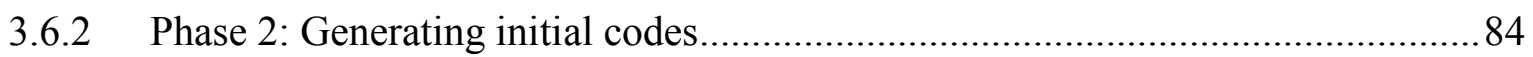

3.6.3 Phases 3 - 6: Identifying and reporting themes ............................................... 86

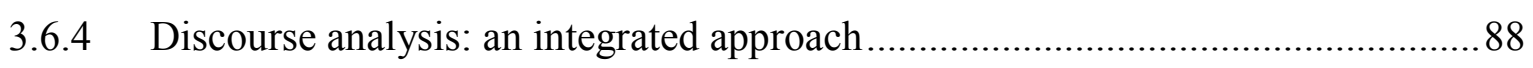

3.7 Credibility of qualitative findings: quality, rigour and validity ................................. 92

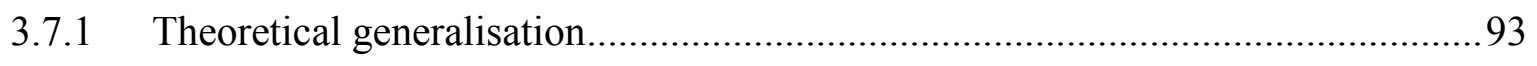

3.7.1.1 Theoretical generalisation and grounded theory ......................................... 94

3.7.1.2 The relationship between discourse and the social world ..............................96

3.7.1.3 Controversy surrounding theoretical generalisation in qualitative research .....99

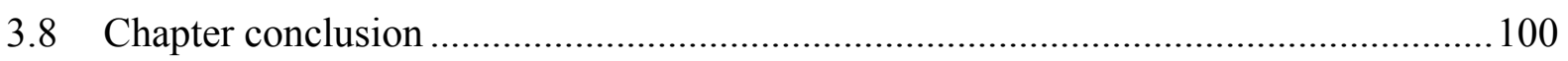

Chapter 4: Misaligned expectations in negotiating service structures and interfaces: a complex systems effect

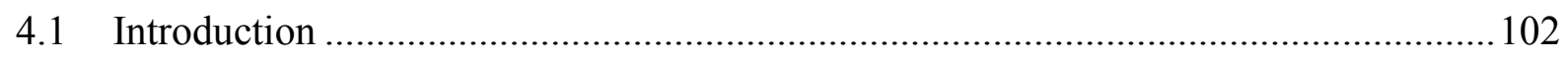

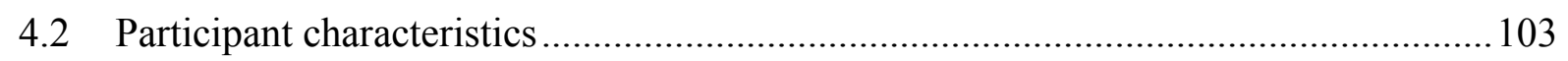

4.3 Construction of the theme: "Misaligned expectations in negotiating transforming

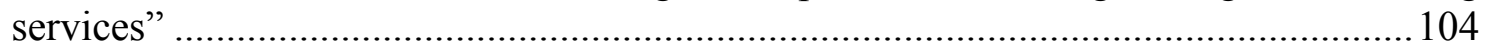

4.3.1 Discharge to primary care across the service system interface .......................... 108

4.3.1.1 "There's not a plan for their future... you don't often see a strapline if things go wrong": Negotiating discharge plan documentation ..................................... 109

4.3.1.2 "All I got told was they were going to discharge me": Being discharged against

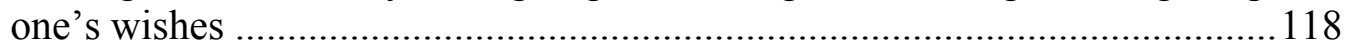

4.3.2 Referral to secondary care across the service system interface.......................... 125

4.3.2.1 "My biggest problem is that I don't have a CPN": Perception of secondary service

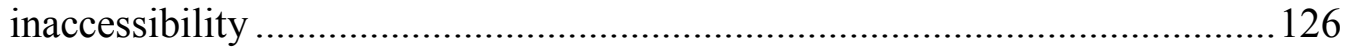

4.3.2.2 "They didn't want to take me back because they said to me that they thought it would be a setback for me": Service boundaries or over-expectant self-referral

4.4 Conclusion

140

Chapter 5: Competing versions of recovery constructed in participants' talk about service experiences

5.1 Introduction 142

5.2 Construction of the theme 'Competing versions of recovery' 143 
5.2.1 Recovery approaches and constructions 143

5.2.2 "It's a bit ambiguous that word, isn't it?" Recovery, a polyvalent concept generating competing versions 145

5.2.3 "Recovery for me would be where I feel well enough to work productively": Competing versions of recovery inhibiting experienced continuity.... 156

5.3 Third sector recovery care and support 161

5.3.1 "It's like children, both parents have got to be stable, and that's the core thing": Recovery versions in third sector care 163

5.4 Third sector collaboration with primary care 168

5.4.1 "I suppose that's helping people towards recovery isn't it?" Oppositionality between third sector recovery versions and primary care . 173

5.4.2 "I think there's a very limited understanding of that specific definition but the ethos of the definition is definitely there": Nascent versions of primary care recovery competing with third sector recovery versions 178

5.5 Conclusion 181

\section{Chapter 6: Experienced discontinuities within primary mental healthcare}

6.1 Introduction 183

6.2 Experiences of practices promoting continuities of primary care

6.2.1 "The only doctor I now see is my GP, and he's been very good": Ostensive continuities masking discontinuities of care 186

6.3 Experiences of practices promoting discontinuities of primary care 193

6.3.1 "Well, they pass the buck then, don't they?" Expectations of the GP role limiting care continuity ..... 195

6.3.2 "They don't have enough insight into mental health. They're not trained psychiatrists": Care discontinuities related to limitations in GP mental health expertise 201

6.3.3 "We want people to be self-empowered and do self-management, but for many people taking away the CPN is very scary": Two competing versions of recovery at the primary and secondary levels 204

6.4 Self-management recovery within primary care .209

6.4.1 "I think there's some good examples and there's some not so good examples": Experienced continuities and discontinuities for self-managed care at the primary level.

6.4.2 "You need to see a doctor, and sometimes you can be desperate": Lived experience of discontinuities within self-managed primary care .214 
6.5 Conclusion .217

Chapter 7: Recovery and complexity in mental health services in Wales: a postpsychiatric and Habermasian perspective

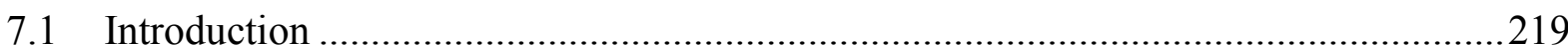

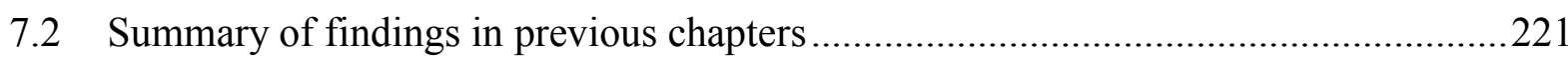

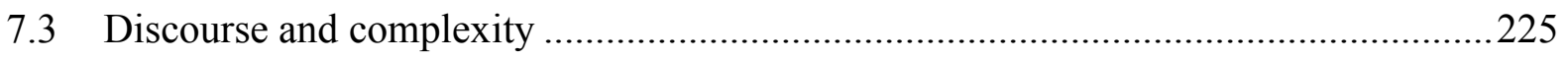

7.4 Discourse complexity and misaligned expectations ...........................................228

7.5 Recovery as a floating signifier generating systemic complexity ..............................233

7.6 Diverse, self-oriented recovery versions contributing to a complex service landscape .236

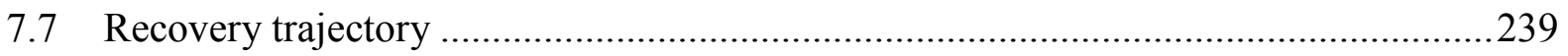

7.8 A postpsychiatric perspective on fragmented mental health services .........................244

7.9 Systemic neoliberalist recovery versions versus lifeworld recovery versions .............248

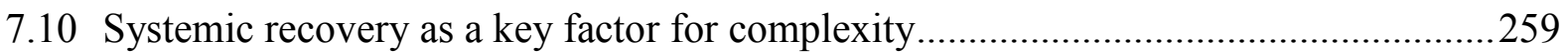

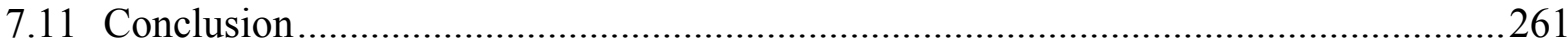

\section{Chapter 8: Study conclusion and implications}

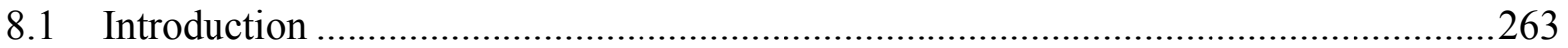

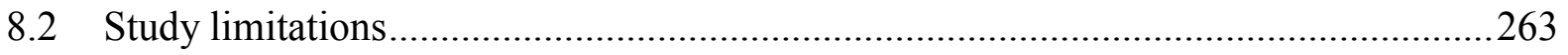

8.3 Theoretical solutions: Ideal speech situations and the public sphere .........................266

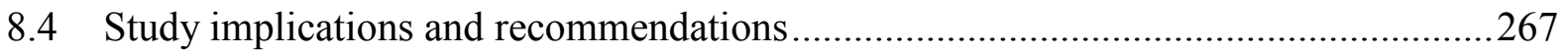

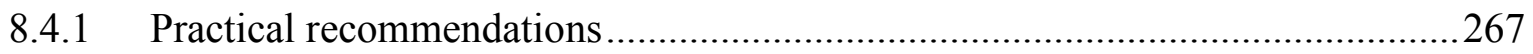

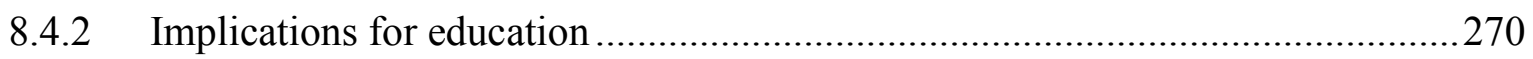

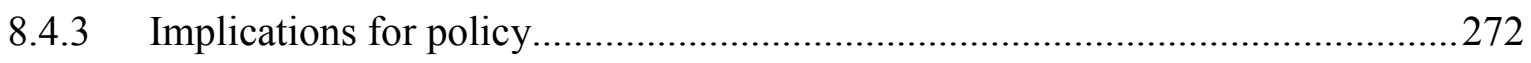

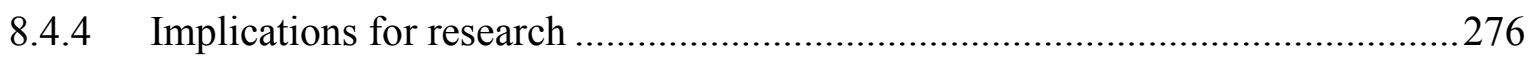

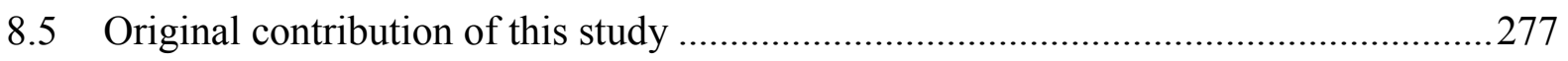

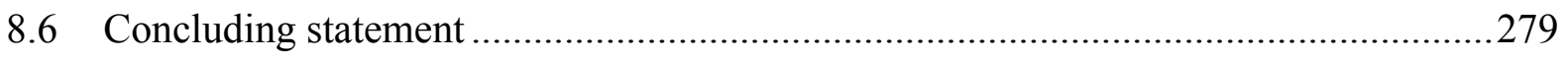

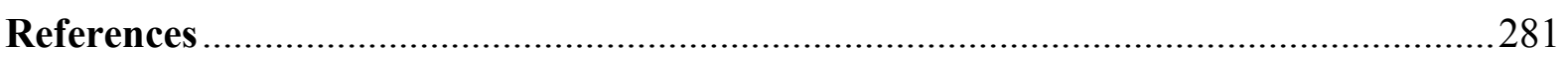




\section{Appendices}

Appendix 1: Interview schedule - Phase 1 - Service users

Appendix 2: Interview schedule - Phase 2 - Service professionals

Appendix 3: Participant information sheet

Appendix 4: Consent form

Appendix 5: Recruitment poster

.321

Appendix 6: Recruitment leaflet

Appendix 7: Participant characteristics.

Appendix 8: Transcribing conventions and symbols

Appendix 9: Inductive search terms and Boolean search strings. .326

Appendix 10: Figure 16: Example of individual participant concept map: Tricia

Appendix 11: Table 6: Summary of findings of studies included in care continuity review 


\section{List of figures}

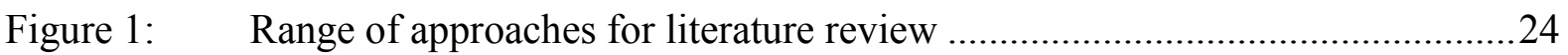

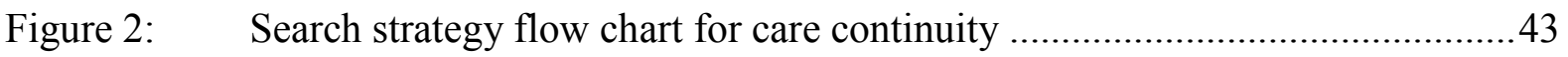

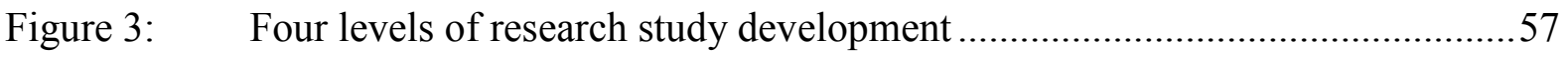

Figure 4: The role of discourse in the constitution of the world....................................96

Figure 5: Discharge to primary care across the service system interface ...................... 106

Figure 6: Referral to secondary care across the service system interface ....................107

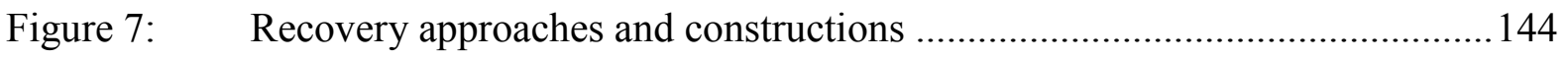

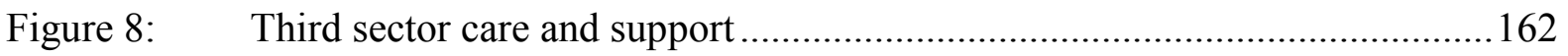

Figure 9: $\quad$ Mental health whole system: a tiered model for adults............................... 170

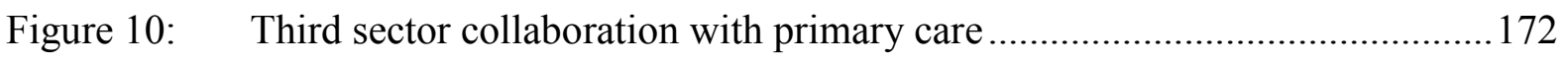

Figure 11: Experiences of practices promoting continuities of primary care .................. 184

Figure 12: Experiences of practices promoting discontinuities of primary care ............. 193

Figure 13: Self-management recovery within primary care .....................................210

Figure 14: Complex service systems effects in Wales .............................................229

Figure 15: Competing recovery versions generating system complexity and service

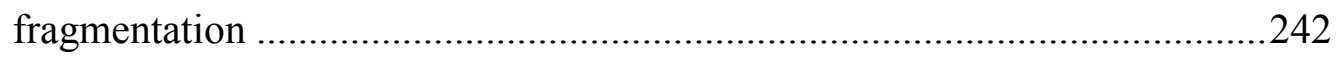

Figure 16: Example of individual participant concept map: Tricia ................................329 


\section{List of tables}

Table 1: $\quad$ Universal quality assessment tool .29

Table 2: $\quad$ Fundamental differences between quantitative and qualitative research strategies .63

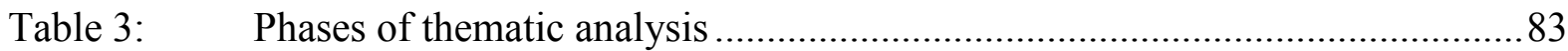

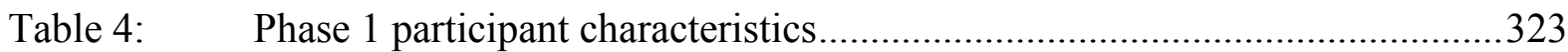

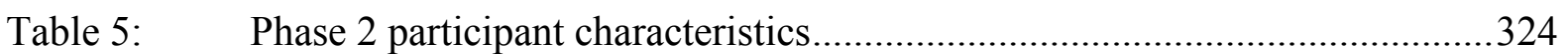

Table 6: $\quad$ Summary of findings of studies included in care continuity review ...............330 


\section{Glossary of terms}

\begin{tabular}{|c|c|}
\hline Care coordinator & $\begin{array}{l}\text { A care coordinator is a mental health professional (such as a mental } \\
\text { health nurse, a social worker, occupational therapist and other } \\
\text { appropriate professions) who in Wales is responsible for co-producing a } \\
\text { Care and Treatment Plan with the service user. }\end{array}$ \\
\hline Care trajectory & $\begin{array}{l}\text { The concept of care trajectory refers to the unfolding process of the } \\
\text { service user's health and illness experiences related to the impact of } \\
\text { surrounding health and social care work and organisations. }\end{array}$ \\
\hline CMHT & $\begin{array}{l}\text { The Community Mental Health Team exists to provide mental health care } \\
\text { for people with SMI (serious mental illness) within the community and } \\
\text { outside of the hospital setting. They are staffed by a range of mental } \\
\text { health occupational professionals and groups, including a psychiatrist, } \\
\text { mental health nurses and social workers. }\end{array}$ \\
\hline CPA & $\begin{array}{l}\text { The Care Programme Approach is the system for care planning and care } \\
\text { coordination introduced in England in } 1991 \text { and in } 2004 \text { in Wales. This } \\
\text { has been replaced by the CTP in Wales which was made mandatory under } \\
\text { the Mental Health (Wales) Measure } 2010 \text {. }\end{array}$ \\
\hline CPN & $\begin{array}{l}\text { Community Psychiatric Nurse, more recently known as Community } \\
\text { Mental Health Nurse (CMHN), is the term used for registered nurses who } \\
\text { provide care for people with mental health problems in the community, } \\
\text { usually as part of the multi-disciplinary CMHT. }\end{array}$ \\
\hline CTP & $\begin{array}{l}\text { The Care and Treatment Plan is a national template in Wales for a care } \\
\text { plan which under the Mental Health (Wales) Measure } 2010 \text { is a } \\
\text { mandatory requirement for coordination and planning of care for people } \\
\text { within secondary services. The template is divided up into eight areas } \\
\text { which are accommodation; finances; spiritual/cultural/social activities; } \\
\text { work and occupation; medical and other treatments including }\end{array}$ \\
\hline
\end{tabular}




\begin{tabular}{|c|c|}
\hline & $\begin{array}{l}\text { psychological therapies; parenting and caring relationships; education } \\
\text { and training; and personal care and physical wellbeing. }\end{array}$ \\
\hline GP & $\begin{array}{l}\text { A General Practitioner is a qualified medical practitioner working in } \\
\text { primary care for both physical and mental illness. }\end{array}$ \\
\hline LPMHSS & $\begin{array}{l}\text { Local Primary Mental Health Support Services are delivered jointly by } \\
\text { health boards and local authorities in partnership with a number of GP } \\
\text { practices in Wales. They support and carry out various enhanced mental } \\
\text { health practices at the level of primary care, as well as providing support } \\
\text { and assistance for mental health services at the primary level. }\end{array}$ \\
\hline $\begin{array}{l}\text { Mental Health } \\
\text { (Wales) } \\
\text { Measure } 2010\end{array}$ & $\begin{array}{l}\text { This piece of legislation was implemented by the Welsh Government in } \\
2012 \text { to support the recovery and care coordination of people with mental } \\
\text { health problems in Wales. It is divided into four main parts. Part } 1 \text { is } \\
\text { intended to improve local primary mental health services. Part } 2 \\
\text { implements the mandatory requirement that people who are treated in } \\
\text { secondary services must have a Care and Treatment Plan. Part } 3 \\
\text { introduces a 'safety net' to counter people falling into a 'gap' between } \\
\text { primary and secondary levels by providing a means for people to self- } \\
\text { refer back to secondary services within three years of discharge. Part } 4 \\
\text { provides for an expanded statutory scheme of mental health advocacy, } \\
\text { including those in hospital informally. }\end{array}$ \\
\hline MDT & $\begin{array}{l}\text { A multidisciplinary team combines health and social care professionals } \\
\text { to collaboratively produce complex care. }\end{array}$ \\
\hline Primary care & $\begin{array}{l}\text { Primary care services are the first point of contact in the healthcare } \\
\text { system including general practice, community pharmacy and local } \\
\text { primary mental health support services (LPMHSS). Traditionally, the } \\
\text { role of primary mental health care has been that of gatekeeping for } \\
\text { specialist secondary mental health services. }\end{array}$ \\
\hline PROM & $\begin{array}{l}\text { A Patient Reported Outcome Measure is a measurement tool which seeks } \\
\text { to ascertain patients' views of their symptoms, functional status and }\end{array}$ \\
\hline
\end{tabular}




\begin{tabular}{|c|c|}
\hline & $\begin{array}{l}\text { quality of life in various domains. They may be divided into two types: } \\
\text { disease specific and generic. They are validated through procedures of } \\
\text { psychometric testing. }\end{array}$ \\
\hline Recovery & $\begin{array}{l}\text { Recovery in mental health is a polyvalent term referring to a constellation } \\
\text { of approaches, ideas and values surrounding mental health care and } \\
\text { wellbeing which is not necessarily preoccupied with achieving cure. } \\
\text { Approaches within recovery may include empowerment and self- } \\
\text { management, a sense of personal journey, establishing hope and new } \\
\text { meaning for life, and social inclusion. }\end{array}$ \\
\hline Secondary care & $\begin{array}{l}\text { Secondary mental health services provide specialist support for people } \\
\text { with complex mental health issues either through hospital or the } \\
\text { community mental health team }(\mathrm{CMHT}) \text {. }\end{array}$ \\
\hline Service user & $\begin{array}{l}\text { A person who is a patient, client or user of health and / or social services, } \\
\text { receiving primary or secondary mental healthcare. }\end{array}$ \\
\hline SMI & $\begin{array}{l}\text { Serious mental illness may be defined as a mental health problem } \\
\text { resulting in serious functional impairment which substantially interferes } \\
\text { with major life activities. It may be distinguished from common mental } \\
\text { illness, where interference with life activities is less severe, though this } \\
\text { point of distinction may be contentious or difficult to specify. }\end{array}$ \\
\hline Third sector & $\begin{array}{l}\text { The third sector or voluntary sector is an umbrella term that covers a } \\
\text { range of different organisations that occupy the 'third' sector after the } \\
\text { public and private sectors. These organisations are non-profit-making } \\
\text { and motivated by charitable concerns or a desire to create social impact } \\
\text { such as in areas of mental health support and recovery. The profile of the } \\
\text { third sector has recently and controversially been raised by the focus of } \\
\text { political visions such as the 'Big Society' based on core themes such as } \\
\text { empowering communities and promoting a culture of volunteering. }\end{array}$ \\
\hline
\end{tabular}




\section{Chapter 1: Introduction}

\subsection{Introduction to the thesis}

This study is about experiences of healthcare and recovery for people with serious mental health issues (SMI) who have recently been discharged from specialist, secondary services to primary care in Wales. Recent service structure transformations in Wales have led to greater movement in both directions between specialist, secondary services and primary care. These transitions can cause disruptions to care trajectories, hinder recovery, and impede opportunities for collaboration between individuals, professionals and service sectors. However, there is little research about the experiences and perspectives of service users and workers in Wales seeking to negotiate these shifting service structures and interfaces. This study aims to investigate these perspectives and generate new knowledge about the impact of service changes on individuals' lives and paths of recovery.

In this introductory chapter, I first outline the study context and background which leads onto the rationale for undertaking this inquiry. Since my chosen research approach is that of qualitative inquiry ${ }^{1}$, I will describe how I 'locate myself' as a reflexive researcher within the study. I present study research question, aims and objectives after these sections since I view them as having emerged logically from consideration of study context and rationale. I conclude this chapter with the final section setting out the structure and organisation of the thesis.

\subsection{The study context and background}

Mental illness is common, with one in four people in the world affected by mental or neurological disorders at some point in their lives (WHO, 2001b). For instance, at a global level, depression is estimated to affect 350 million people (WHO, 2012), and is predicted to be the second most common cause of disability after ischaemic heart disease by 2020 (WHO, 1999). Mental health and illness are shaped to a great extent by social and economic determinants, as well as by health and social inequalities occurring across the lifespan (WHO,

\footnotetext{
${ }^{1}$ In section 3.3 I will describe my methodological rationale for undertaking a qualitative approach for this research inquiry.
} 
2014). At the same time, mental health issues are complex, affecting many areas of individuals' lives, as well as their status within a wider social context (Bracken et al., 2012; Crawford et al., 2004; Rogers and Pilgrim, 2014). Conditions are often chronic, and greatly affect relationships, social inclusion, housing, education and social capital (Amering and Schmolke, 2009; Keetharuth et al., 2018; Putnam, 1995; Tew et al., 2011). In the economic sphere, illness may adversely affect employment and economic activity, with detrimental knock-on effects for the wider economy. Mental illness is costing the UK more than $£ 94 \mathrm{bn}$ each year according to the OECD (2018), based on costs of treatment, social support and lost employment. More locally, in Wales, the cost of mental illness in monetary terms has been estimated at $£ 7.2$ billion in 2007/08 (Friedli and Parsonage, 2009).

Despite this, the treatment gap for mental health problems is enormous in all countries of the world (WHO, 2008). There is a significant gap between the prevalence of mental illness and the availability of treatment and care (WHO, 2008). In the UK, long-term deficits in financial investment into mental health services have been heightened by the impact of austerity with funding cuts of $8 \%$ in real terms since 2010 (McNicoll, 2015). In Wales, the need for fiscal stringency and more frugal economic planning is supported by prudent health care principles based on reducing inappropriate variation through evidence based approaches, focusing resources on individuals with the greatest need, reducing iatrogenic harm (Illich, 1993), and facilitating co-production (Aylward et al., 2013; see also http://www.prudenthealthcare.org.uk/principles/). With a certain degree of incongruence, this culture of fiscal stringency has been promoted under the rhetoric of prioritising mental health as both a policy and public health concern (Health and Social Care Act, 2012; HM Government, 2011; Welsh Government, 2012b). This disparity between rhetoric and action has occurred against a backdrop of successive programmes of service transformation in recent service history including Wales (Welsh Government, 2010b; 2010c), a legacy which some view as having had an overall detrimental effect on mental healthcare delivery (Braslow, 2013; Gilburt et al., 2014; Gilburt, 2015; Hannigan, 2013; Hannigan and Coffey, 2011). These programmes have involved the shift from institutional to community-based care starting in the $1960 \mathrm{~s}$, culminating in England with the introduction of the Care Programme Approach in 1991 (Gilburt et al., 2014; NHS and Community Care Act, 1990). This was introduced in the light of service failures and tragic consequences, such as the killing of Jonathon Zito (Coid, 1994; 
Court, 1994; Ritchie et al., 1994; Simpson et al., 2003; Simpson, 2005). Further policy initiatives for reformulating service approaches have centred upon supplementing standard community mental health teams (CMHTs) with newer services providing more specialised support. These have included crisis resolution and home treatment (CRHT), assertive outreach, and early intervention for people with psychosis (DoH, 1999; DoH, 2001b; Hannigan, 2013). More recently, an influential approach that has emerged from the mental health service / user survivor movement has been 'recovery' (Glasby and Tew, 2015). Originating with service users in the 1980s and 1990s (Anthony, 1993; Deegan, 1988; Deegan, 1993), the concept of recovery has grown into one of the most important paradigms ${ }^{2}$ and prominent rhetorics within modern mental health services (Edgley et al., 2012; Morrow, 2013; Pilgrim and McCranie, 2013; Woods et al., 2019). It may now be considered to be the contemporary paradigm for mental health services and policy implementation (Braslow, 2013; Edgley et al., 2012; Hannigan et al., 2018), and occupies the forefront of many policy agendas in a variety of countries around the world (Bonney and Stickley, 2008; Braslow, 2013; Morrow, 2013; Pilgrim and McCranie, 2013; Welsh Government, 2014c).

At a broad policy-level, these successive transformative programmes have attempted to identify and resolve key areas of system failure (Hannigan and Coffey, 2011). Actions have aimed at repeated reformulation or re-casting of the problem of mental healthcare policy

\footnotetext{
${ }^{2}$ In this thesis I use the concept of 'paradigm' on a number of levels. A paradigm may be defined as a set of basic beliefs or a worldview that determines, for its holder, the nature of the world and the individual's range of possible relationships to that world and its parts (Guba and Lincoln, 1994).

In classing recovery as a paradigm, I understand it to be a particular set of beliefs constituting a worldview for looking at mental illness and healthcare as it occurs amongst individuals who make up society. This paradigm therefore operates at a sociological or cultural level (Edgley et al., 2012; Morrow, 2013).

Later in this chapter, I describe perspectivism as a paradigm operating at a philosophical level since this describes at the most basic level the epistemological relationship between the researcher and the world for this study. The multi-perspectivist view in healthcare described by Heaton and associates (2012) and Sweeney and colleagues (2016), which I employ in my study design (see section 3.2), is one version of this perspectivism.

In Chapter three, I refer to social constructionism as a paradigm. This paradigm operates at the level of methodological inquiry for this study, as described by Guba and Lincoln (1994): "Inquiry paradigms define for inquirers what it is they are about, and what falls within and outside the limits of legitimate inquiry" (p.108). I therefore employ the concept of paradigm at three levels in this thesis: the philosophical level, the methodological level and the sociological level.
} 
through successive implementation of top-down standards, new service frameworks and clinical guidelines (Gilburt et al., 2014; Hannigan and Coffey, 2011). Gilburt and associates (2014) note that, as a consequence of these initiatives, traditional dividing lines between various sectors of these services have fragmented, implying that health care continuity is likely to be disrupted. Fragmented arrangements are one legacy of successive changes to mental health systems and hinder integrated treatment provision (Gilburt et al, 2014). Care continuity, which may be considered to be a benchmark for healthcare systems appraisal (Burns et al., 2009; Schultz and McDonald, 2014; Sweeney et al., 2016; Weaver et al., 2017), is therefore a key framework within which service provision and fragmentation may be appraised.

The number of policy initiatives has been multiplied by the opportunity for divergent healthcare approaches and frameworks afforded by devolved administrations in the UK, (DoH, 1999; DoH, 2007; Scottish Executive, 2006; Welsh Government, 2005; 2011b; 2012b). In Wales, the Welsh Government has chosen to use legislation as the primary driver for improvement in mental health delivery, recovery-based services and care continuity between various service sectors (Glasby and Tew, 2015). The context for this legislation has been a broader crossgovernmental mental health and wellbeing strategy for Wales called 'Together for Mental Health' (Welsh Government, 2012b). This sets out a new partnership with the public, aimed at promoting better mental health and wellbeing, increasing service user and carer involvement, tackling stigma and discrimination, and an underlying commitment to recovery-oriented services (Welsh Government, 2012b).

In this strategic context, the Mental Health (Wales) Measure 2010 has tidied up pre-existing legislation, focusing on augmented primary mental health delivery, and enhanced care coordination and care planning at the level of specialist, secondary services. The Measure has placed Wales in a unique position within the UK, since it seeks to implement primary mental healthcare delivery, underpinned by recovery values, at a policy-level (Hannigan et al., 2018; Welsh Government, 2011a; 2013; 2014c). This is designed to provide a legal framework to improve mental health services in Wales through strengthening the role of primary care within mental healthcare (Welsh Government, 2011a; 2015). In addition to this, at the secondary level, 
care planning is carried out through mandatory use of a national template in the form of the Care and Treatment Plan (CTP) (Hannigan et al., 2018; Welsh Government, 2015).

The Welsh Measure is divided up into four main parts. Part 1 of the Measure is intended to improve local primary mental health services by introducing enhanced practices such as making available comprehensive mental health assessments, short term treatments such as brief Cognitive Behavioural Therapy (CBT), and greater support for referral to secondary mental health care (Welsh Government, 2011a). Part 1 also attempts to facilitate within primary care a greater provision of information about treatment available to allow a higher level of signposting to other services (Welsh Government, 2011b). The Measure seeks to establish Local Primary Mental Health Support Services (LPMHSS), delivered jointly by health boards and local authorities in partnership with a number of GP practices (Glasby and Tew, 2015). These support and carry out many of the above enhanced practices, as well as providing specialist support and assistance for mental health services at the primary level (Welsh Government, 2014c).

Part 2 of the Measure implements the mandatory requirement that people with SMI who are treated in secondary services must have a Care and Treatment Plan (CTP) and a carecoordinator (Hannigan et al., 2018; Welsh Government, 2010b; 2011a). This initiative seeks to provide all individuals receiving secondary mental health services with an holistic CTP underpinned by recovery-values (Welsh Government, 2010a; 2014c; 2015). I argue that this has been an indirect factor contributing to the shift towards primary mental healthcare in Welsh services, due to its effect of an increased level of discharges from secondary services. The mandatory requirement for patients at the secondary level to have a CTP and care coordinator means that people held on psychiatrists' outpatient lists now need to be care coordinated. However, the idea that psychiatrists become care coordinators was challenged and ultimately not fulfilled by the Royal College of Psychiatrists in Wales, since this role was seen not to fit the medical model governing their practice (RCPsych, 2014). At the same time, community mental health teams did not want to take these extra patients onto their caseloads since they were already under significant workload pressures (ORS, 2014). The care coordinator can be one of a limited number of trained mental health professionals, predominantly Community 
Psychiatric Nurses (CPNs) (Welsh Government, 2012a), and this has proved to place a significant burden on CMHTs in Wales to accommodate the work needed, in comparison for instance to the CPA approach in England. There is also evidence that formalising care planning through legislation and the introduction of increased scrutiny has led professional staff to be unwilling to take on the role of care coordinator (ORS, 2014). The overall result of these factors was that a number of people with SMI, who had previously been retained within secondary services, were discharged to primary care (Gofal, 2014; 2015), whilst a more select group of people with SMI would continue to be retained at the secondary level, for whom this level of care was deemed appropriate. This can be considered to correspond to the prudent healthcare principle of focusing resources on individuals with the greatest need (Aylward et al., 2013). Although it was one of the stated aims of the Measure that "discharge from specialist care (such as secondary mental health services) is regarded as a key outcome of the recovery model within mental health" (Welsh Government, 2010a, p.42; 2013, p.11; 2014c, p.15), the process I have described has led to the number of discharges being greater than anticipated. Emerging evidence is illustrative of this process and its impact, which is considered to be an unforeseen consequence of implementation of the Mental Health (Wales) Measure 2010 (Gofal, 2014; ORS, 2014; Welsh Government, 2014c).

Part 3 of the Measure introduces a "safety net" (Welsh Government, 2014c, p.18) to counter people falling into a 'gap' between primary and secondary levels by providing a means for people to self-refer back to secondary services within three years of discharge (Welsh Government, 2014b; 2015; 2016). This is intended to be a key provision for people who have been discharged to primary care since it should allow them to access specialist care quickly and directly if their mental health deteriorates.

Part 4 of the Measure, which I did not deem to be relevant to the focus of this study, provides for an expanded statutory scheme of mental health advocacy, including those in hospital informally (Welsh Government 2011a).

The Mental Health (Wales) Measure can be considered to be underpinned by recovery values in two ways. Part 1 of the Measure emphasises self-management and independence though 
increased treatment within primary care, along with reduced dependency upon secondary services (Welsh Government, 2010a; 2013; 2014c). Part 2 of the Measure emphasises selfmanagement in collaboration with the care coordinator and an holistic approach under the eight areas of the $\mathrm{CTP}^{3}$ (Welsh Government, 2014c; 2015; 2016). However, it is important to emphasise that recovery is a wider phenomenon than any particular policy implementation such as the Mental Health (Wales) Measure. Policy may be an important context and even a catalyst for trends towards recovery-values, but should be considered to be an attribute of the paradigm rather than constitutive of it. Therefore, this study is not about the impact of the Mental Health (Wales) Measure 2010 but has a broader focus upon the experiences and perspectives of service users and workers in Wales as they negotiate transforming services within the contemporary mental healthcare paradigm of recovery (Braslow, 2013; Edgley et al., 2012; Hannigan et al., 2018). However, the policy is an important context and background for this research focus, which is why I have provided details here.

Illustrating this point, recovery-implementation in the form of increased self-management after discharge to primary care, which features under Part 1 of the Measure, is a much wider, global phenomenon (WHO, 2008). This is the case whether it is captured in the form of legislation, or is the result of cultural trends towards augmented primary mental healthcare delivery (WHO, 2008). Traditionally, service models for people with serious mental illness have been dominated by secondary care services (Gilburt et al., 2014; WHO, 2008). The role of primary mental health care has been that of gatekeeping for specialist secondary mental health services (Currid et al., 2012). More recently, it has been argued that primary care is now sufficiently mature as a discipline to take on a lead role in mental health service delivery (Currid et al., 2012; Green and Thoroughgood, 1998; Lester et al., 2004; WHO, 2008). An increased emphasis on primary mental healthcare delivery may also be related to a recovery approach aimed at reducing specialist service dependency and service provision (Lester and Gask, 2006; Ramanuj et al., 2015). Another key driver behind this transition is the notion that primary care

\footnotetext{
${ }^{3}$ These eight areas are accommodation; finances; spiritual/cultural/social activities; work and occupation; medical and other treatments including psychological therapies; parenting and caring relationships; education and training; and personal care and physical wellbeing (Hafal, 2012; NHS Wales, 2018).
} 
is more cost-effective than secondary or hospitalised care (Ramanuj et al., 2015; van Dijk et al., 2014).

Despite these mitigating points, there are a number of challenges facing the vision of augmented primary mental health services (Durbin et al., 2016). Like many areas of healthcare in the NHS, the burdens already placed upon GPs and primary care workers are considerable, involving significant time and workload pressures leading to occupational stress (Wilkinson, 2015). Given the complexity of mental health treatment, practitioners are often reluctant to take on the new level of responsibility engendered by an increased emphasis on mental health (Freeman et al., 2002; Gofal, 2014; WaMH in PC, 2015). Many perceive expanding their involvement in the field as being like opening 'Pandora's box' due to the complex nature of this kind of healthcare (Fleury et al., 2012; Lester, et al., 2004; WaMH in PC, 2015).

A recent study in south London highlighted difficulties facing increased primary care for people with SMI (Ramanuj et al., 2015). This study showed that $58.2 \%$ of people discharged from secondary to primary care were referred back to secondary care within two years of their original discharge (Ramanuj et al., 2015). Of these individuals, 60.3\% were experiencing a crisis in their mental health condition at the time of referral. The high proportion of people referred back to secondary care within two years, many of whom were at a point of crisis, suggests that there may be inadequacies in mental health care at the primary level. Ideally treatment should be delivered as an early intervention prior to the person reaching the point of crisis, since this can often improve prognosis and may prevent future need for hospital admission (Rhodes and Giles, 2014). The study by Ramanuj and colleagues (2015) appears to indicate that early intervention strategies are not being implemented adequately at the primary level. In addition to problems with service delivery within primary care, shortfalls may also be due to interruptions in care continuity at the point of transition across the interface between secondary and primary levels.

The difficulties encountered in the study by Ramanuj and associates (2015) may have greater applicability to the Welsh context, given that there is a legislative driver at play in the form of the Mental Health (Wales) Measure 2010. Indeed, a recent report from a special interest 
working group of the Royal College of GPs in Wales highlighted 72.7\% of primary care staff as describing mental health as 'difficult' or 'very difficult' to manage (WaMH in PC, 2015). One investigation into primary mental health delivery by a third sector organisation identified a 'stubborn' 20-25\% of GPs who are regarded by service users as only 'slightly' or 'not at all' understanding and empathetic concerning mental health issues (Gofal, 2014, p.3). In Wales, preliminary studies into the impact of the Measure have identified care continuity issues associated with service transformation (ORS, 2014). Concerns have been raised in relation to people who are caught between primary and secondary services, whose conditions are considered too severe for primary care services but not severe enough for secondary services, and who consequently have not been able to access treatment at either level. This experience has been described by service users with terms like 'bouncing' between sectors (ORS, 2014, p.4, p.35) and as being "passed from pillar to post" (Gofal, 2013, p.31).

It has been suggested that a fundamental factor behind these difficulties is that primary care practitioners do not have the right set of skills or level of expertise to cope with people with SMI who have moved from specialist services (Thielke et al., 2007). Important activities associated with the care of people with SMI, such as case management and follow-up may be lacking at the primary level. Additionally, primary care is focused on the management of acute diseases and maintenance of public health, which does not match treatment of mental illness often rooted in complex sociological factors (Thielke et al., 2007; WHO, 2014). These issues are potentially a cause for concern when considering current trends and policy programmes in Wales towards augmented primary mental healthcare as a form of recovery implementation.

The history of mental health services in the UK has been described as a series of transformations intended to implement beneficial changes to services, workforce and infrastructure (Gilburt et al., 2014; Gilburt, 2015). Though these transformations have often engendered financial-savings, they have often come at the cost of patient care (Gilburt, 2015). A key question therefore is whether the shift to primary mental healthcare under the auspices of recovery will lead to improvement in services or whether this is the latest in the succession of cost-saving transformations which may paradoxically result in poorer quality care and treatment. 


\subsection{The rationale for the study}

Summing up key points from the previous section on the study context, there are a number of issues facing the care and treatment of people with SMI, who have been discharged to primary care in Wales (Lester et al., 2004; Ramanuj et al., 2015; Thielke et al., 2007). There is evidence that the Mental Health (Wales) Measure 2010 may have stimulated a greater than expected level of discharges to primary care (Gofal, 2014; ORS, 2014; Welsh Government, 2014c), and that services may be poorly prepared for these individuals with SMI (Gofal, 2013; Gofal, 2014; Gofal, 2015; ORS, 2014). These issues are potentially associated with disruptions to care continuity and care coordination, and a negative impact on individuals' experiences of recovery (Gilburt et al., 2014). There is an absence too of first-hand accounts of individuals experiencing discharge from secondary mental health care to primary care and therefore little evidence of the day to day realities of what this involves.

These legitimate concerns can be seen to provide good grounds for undertaking an investigation into the care continuity and recovery experiences of people with a history of SMI, who have been discharged to primary care in Wales. This is particularly the case given the lack of independent scholarly research into the condition of contemporary Welsh mental health services, and the associated impact of recent devolved policy (Gofal, 2013). There is currently very little academic research and literature on the Mental Health (Wales) Measure and its implementation, which is an important context and background for the research focus. Currently, only non-academic surveys and reports have been published examining in detail the implementation of the Measure, either by the Welsh Government (2014b; 2015; 2016), on behalf of the Welsh Government (ORS, 2014), or by independent organisations (Gofal, 2013; 2014; 2015; WaMH in PC, 2015). There can therefore be considered to be a gap in scientifically valid knowledge concerning the Mental Health (Wales) Measure 2010 and its impact on experiences of recovery and care continuity at the interface of primary and secondary care (Gofal, 2013). There can also be considered to be a gap in knowledge in general concerning perspectives of service users and workers in Wales seeking to negotiate shifting service structures and interfaces, with the Measure as an important background for this. This is an important gap in knowledge which this study seeks to investigate. Therefore, this research project aims to investigate patient and worker perspectives, particularly after discharge to 
primary care, and generate new knowledge about the impact of service changes on individuals' lives and paths of recovery.

\subsection{Locating myself within the study}

As well as considering the social and policy background for the study, the researcher should also be considered to be an important context for the research inquiry. For this reason, the researcher should always locate him/herself within the study (Etherington, 2004; Riessman, 1993). Study findings will be affected by the cultural biases, values and perspectives brought by the researcher to the inquiry (Guba and Lincoln, 1994). In this way, the researcher is seen as an active agent producing meaning and knowledge about the world in the process of coconstructing it with participants (Denzin and Lincoln, 1994; Hewitt, 2007).

Therefore, the researcher must practice reflexivity. Reflexivity may be understood as an ongoing process whereby researchers critically self-examine through self-conscious reflection on their own role in contributing to the data collected, and the shaping of analysis (Finlay, 2002; Hewitt, 2007; May and Perry, 2013). The researcher should therefore identify themselves as an important context for the research inquiry, with a particular position and perspective in relation to research phenomena. Both perspective and positionality can only properly be understood by reflecting on the researcher's cultural biases, values and interpretations which emerge from his or her social, political and historical context (Bryman, 2016; Guba and Lincoln, 1994). As such, all knowledge is a reflection of the researcher's location in a cultural, political and social context as this is brought to bear on the study focus.

The notion of reflexivity is contested as being subjective, ambiguous and woolly (Finlay, 2002; Lynch, 2000). Certainly, it can be considered a difficult concept to understand and to practice. Advocates of reflexivity warn against a kind of introspective navel-gazing, in which excessive self-analysis is carried out at the expense of focusing on research participants (Finlay, 2002; Hewitt, 2007). In order to tackle these difficulties, I adopted four key questions provided by Kim Etherington (2004) which the researcher should ask him/herself as they undertake their study: 
- "How has my personal history led to my interest in this topic?

- What are my presuppositions about knowledge in this field?

- How am I positioned in relation to this knowledge?

- How does my gender/social class/ethnicity/culture influence my positioning in relation to this topic/my informants?"

(Etherington, 2004, p.16)

I will now deal with each of these questions in turn, starting with a brief account of my background which led to my personal interest in undertaking this project. I also provide some initial considerations of how this might affect my perspective on research phenomena, which addresses to a certain extent the second, third and fourth questions provided by Etherington (2004). I started training as a mental health nurse in 2011, coming from a background of working as a support worker in a third sector organisation which provides residential treatment for people with addiction problems. During this time, I encountered at first hand the great difficulties people can experience in trying to overcome life-controlling problems, which can extend over a protracted period of time and potentially end in fatalities. Most of the residents had had some kind of (often traumatic) encounter with mental health services, since dualdiagnosis (APA, 2000) was commonplace, meaning that they had often been admitted voluntarily or involuntarily to acute or secure units during psychotic episodes. Additionally, I considered that the various addiction and dependency issues that clients presented with should be considered to be mental health problems in their own right, a view which is supported by contemporary conceptions and taxonomies of mental illness (APA, 2013; WHO, 2014). More common mental health problems such as depression and anxiety were also frequent supporting co-morbidities for alcohol and drug dependencies.

This work led to the development of my interest in the concept of 'recovery' which was used as a generic term by clients to describe the various approaches and pathways which they undertook in tackling their mental health and addiction issues (Senker and Green, 2016). I also saw the crucial role of continuous, good quality care in supporting recovery. Hiatuses or 
impasses occurring within care trajectories potentially had disastrous results. For instance, individuals leaving the rehabilitative programme prematurely or in an unplanned way would often lead to full-blown relapses and potentially fatalities due to overdose. These experiences crystallised my interest in recovery, an approach that was often ill-defined but grasped by clients as something fundamentally important for their progression out of addiction and towards a better state of mental health and wellbeing (Anthony, 1993; Deegan, 1988; Senker and Green, 2016). This was also the origin of my comprehension of the concept of care continuity and the relationship it has with recovery. I would later develop a much fuller understanding of care continuity in my published systematic review (Weaver et al., 2017), which I have applied within this study.

These various features of my background led both to my interest in the project and also potentially to certain presuppositions in relation to it. Many of the individuals I supported had had negative or inconsequential experiences of medical and psychiatric sector support, which had led them to seek alternative help through third sector organisations, such as the one I worked for. The third sector provides support for individuals potentially as an alternative to services, and is therefore one source of a critical view of services which may be seen by clients and workers alike as having failed them in some way. I considered that this prior experience working in the third sector might have engendered in me a certain collusion with service users' and third sector worker participants' complaints about services.

This critical view of services was compounded by my experiences after graduating when I worked as a nurse on an acute psychiatric ward. Here, pressures on services were considerable which led to depleted opportunities for sustained, one-to-one high-quality care for patients and for care planning to be properly accomplished. It appeared to me that services were pinned down in a state of perpetual 'firefighting' (McNicoll, 2013), exacerbated by the unnecessary burden of excessive nursing paperwork which did not necessarily contribute in a real way to effective, collaborative care planning and provision (Coffey, et al., 2017b; McKeown et al., 2017; Simpson et al., 2016b). This scenario was overlaid with a prevalent and jaded ethos that acute care should be as short as possible since care should be community-focused. What this amounted to in practice was that staff at all levels would try to discharge patients as soon as 
they were admitted meaning that they would effectively receive minimalistic treatment or even no treatment at all. This was at a time which might otherwise be an opportunity for crucial intervention in an acute setting, having reached a point of crisis out in the community. 'Revolving-door' patients (Freeman et al., 2002) were therefore commonplace as were other more negative outcomes from the patient's point of view.

I reflected that the combination of these presuppositional factors could create a bias which might lead to me colluding with participants' accounts of poor service experiences, during data collection and analysis, especially if their accounts were adroitly constructed to engender sympathy and agreement. This outlook would also potentially be compounded by a negative view of services propagated by the media, and a more general level of cynicism, pessimism and stigmatisation about mental healthcare which is widespread in society (Bowen, 2019; Bowen and Lovell, 2013; Campbell, 2017; Chen and Lawrie, 2017).

Reflective consideration of these, and other, potential biases and presuppositions meant that they could be incorporated into the epistemological ${ }^{4}$ content of the study. By doing this, I believe a more balanced approach to apprehending research phenomena was achieved, which was true to the hermeneutical and perspectival nature of qualitative inquiry. This involved me standing apart from negative accounts to a certain degree, yet at the same time being clear about my own interpretative contribution towards knowledge-creation, based on reflection about my background, and the various views and perspectives which I brought with me to the research process.

I have described the foundation of the reflexive process in this section. However, reflection should be carried out as an ongoing activity throughout the course of the research project (Coffey and Atkinson, 1996). I therefore continued to practice reflexivity by keeping a reflective diary and field notes throughout the project. This reflexive practice was continued

\footnotetext{
4 'Epistemology' may be defined as the 'knowability' of the social world, and the relationship between the knower and what is known (Epstein, 2011; Ritchie et al., 2013). Epistemology may be contrasted with 'Ontology' which concerns the nature or composition of the world; literally its state of being (Epstein, 2011; Guba and Lincoln, 1994; Scruton, 1994).
} 
throughout the stages of data analysis during which I made memos concerning reflexive realisations. The more important considerations emerging from this process of reflection, and how they interacted with data collection and analysis, are discussed at points in subsequent chapters (see for instance section 4.3.2.2 and analysis of Katy's talk).

At this point I would like to add that my $\mathrm{PhD}$ experience was something of a philosophical journey from an initial position approximating to naïve realism (Willig, 2013), to a more sophisticated, interpretative epistemological outlook. This is related in a general way to Etherington's (2004) third question of reflexivity concerning how I was positioned in relation to new knowledge. Clearly, my understanding of my position in relation to knowledge shifted in a fundamental way during the course of the project. My original position of naïve realism and a related positivism may have been partly due to my having been trained as a biomedical nurse within a biopsychiatric worldview. One of the challenges of undertaking this $\mathrm{PhD}$ project was coming to terms with the epistemological character of qualitative inquiry, something which I struggled with at first. I was helped greatly in this journey by study of some of the great interpretivist thinkers such as Wilhelm Dilthey (1976) and Martin Heidegger (1967), with the aid of supportive texts which enabled me to access their thought (Bishop, 2007; Delanty, 2005; Manicas, 2006; Mulhall, 1996). This endeavour helped me to get to the roots of the 'interpretive turn' which had occurred in philosophy and social theory during the $20^{\text {th }}$ century and connect with this on a personal and intellectual level. This enabled me ultimately to be able to fully embrace the epistemological outlook of qualitative research for my study.

In section 3.3, I write about this 'interpretive turn' more extensively in relation to social science and research methodology. This culminates in my adoption of social constructionism based on the underlying principle of 'intentionality' for this project (see section 3.3.4). My personal journey was however the underpinning which enabled me to embrace and apply this methodological position, and was also the basis for my adoption of the fundamental philosophical paradigm of perspectivism as the basis for this inquiry. A paradigm may be defined as a set of basic beliefs or a worldview that defines, for its holder, the nature of the world and the individual's range of possible relationships to that world and its parts (Guba and Lincoln, 1994). I viewed the philosophical paradigm of perspectivism as the underlying 
philosophical or categorical ${ }^{5}$ paradigm for this thesis, because it describes at the most basic level the epistemological relationship between the researcher and the world, which I chose to be a perspectivist or interpretivist viewpoint.

I found that this perspectivist paradigm should also be applied to the relationship between participants and the world as well as to the relationship between the researcher and the world. This accords with the principle of the 'double hermeneutic' which highlights the dual interpretative capacity of both researcher and participant in social inquiry (Giddens, 1984). I drew on the work of Sweeney and associates (2016) who adopt such a perspectivist paradigm in the context of healthcare and care continuity in mental health. A related understanding is the 'new subjective medicine' provided by Sullivan (2003). Here, patient subjectivity is explicitly incorporated into assessments of health and care, so that it is asserted that the most complete and meaningful assessments of health are based on the patient's perspective (Sullivan, 2003). Patients and not the expert clinician are the definitive perceivers of their health state and treatment requirements (Asadi-Lari et al., 2004). I adopted a perspectivist paradigm applied in this way to the context of healthcare as a key philosophical and epistemological underpinning for this study.

Finally, in relation to the fourth reflexive question provided by Etherington (2004), I considered the influence of the feminist perspective on the notion of reflexivity. Here, the abstract masculinity inherent in the authoritative viewpoint of positivism is contrasted with the connectedness and continuities of women exemplified through the ethics of empathy and care (Fine, 2002; May and Perry, 2013). The latter view relates to a more reflexive and interpretivist approach to knowledge and understanding which I ultimately embraced for this project in the form of the perspectivist paradigm. The feminist influence on this interpretivist position may go some of the way to explaining why, as a man, I struggled at first to move from a naïve realist, positivist epistemological stance to a more perspectivist view.

\footnotetext{
${ }^{5}$ Here, I employ the term 'categorical' in the philosophical sense originally employed by Kant (1998 [1781]) to describe an indispensable, a priori framework within which knowledge and understanding must operate (Kenny, 2006).
} 


\subsection{Research question, aims and objectives}

The over-arching research question for this study may therefore be clarified on the basis of the background and context, including that of the researcher, provided in the previous sections:

"What are the experiences of care continuity and recovery for people at the interface of primary and secondary mental health care in Wales?"

The overall aim of this study therefore is to provide new evidence on the organisation and experience of care continuity and recovery at the interface between primary and secondary care in Wales.

Therefore, the study aims and objectives are:

- To produce an evidence synthesis of care continuity and recovery literature relevant to persons with a history of serious mental illness, related to movement across the primary and secondary mental health interface.

- To investigate how individuals experience the organisation and delivery of care continuity and recovery while moving across the interface between primary and secondary sectors as a result of current service transformations and trends in Wales, considering the policy-context of Mental Health (Wales) Measure 2010.

- To investigate these experiences of health and care using a qualitative research approach within a perspectivist epistemological paradigm.

\subsection{Organisation of the thesis}

In this first chapter, I have introduced my study, provided a rationale for the inquiry, and set out my research aims and objectives. I have also described the study context and described the groundwork for 'locating myself' reflexively within the thesis. In Chapter two, I provide further background for the study by review and appraisal of literature relevant to the key concepts of the research inquiry. This points towards gaps in knowledge which are appropriate areas for 
investigation within this study. Review of relevant subject areas also provides a theoretical context for qualitative data analysis. As well as this, I develop an argument related to the subject areas of the review, which forms a rationale for the overall focus of the research inquiry. This argument focuses on mental health services in Wales impacted by policy, understood though complex systems theory, then on recovery as the key focus of policy, and finally on care continuity as a key element in provision of recovery-focused care.

In Chapter three, I build on the background provided in Chapters one and two, by describing the methodology and methods I used to achieve the aim and objectives of the study. Here, I build upon the underlying perspectivist epistemological paradigm to form an interpretivist methodology for sociological inquiry into recovery and care continuity experiences. This involves a reconciliation of social constructionism and critical realism as a way of combining an interpretivist outlook with a notion of objectivity. I also indicate that my study is divided into two phases of data collection. The first phase involves semi-structured interviews with service user participants who have been discharged from secondary services within the past three years. I considered this experience to be relevant to the implementation of the Mental Health (Wales) Measure 2010, since three years is the timescale for the option of self-referral back to secondary care under Part 3 of the Measure (Welsh Government, 2014b; 2015; 2016). The second phase involves interviews with workers and professionals involved and supporting service users fulfilling the inclusion criteria of the first phase of data collection. I then describe details of my method in terms of data collection, data analysis and research ethics. I describe my method of thematic discourse analysis (Braun and Clarke, 2006; Braun et al., 2014; Singer and Hunter, 1999). I also discuss evaluative criteria concerning quality, rigour and credibility of qualitative findings. This leads me to specify theoretical generalisation (Mitchell, 1983; Seale, 1999) as the basis for new knowledge to be generated by this study.

In Chapter four, which is my first findings chapter, I employ complexity theory, introduced in Chapter two in relation to mental health services and the impact of policy, to provide a context for analysis of the talk of participants. This analysis leads to construction of the theme 'Misaligned expectations in negotiating transforming services'. I argue that service structure changes, in particular influenced by implementation of recovery-based services by the Mental 
Health (Wales) Measure 2010, have led to the complex systems effect of misaligned expectations in negotiating the primary-secondary interface. This complex systems effect or 'wave of consequence' (Hannigan and Coffey, 2011) is most manifest in discursive practices, positionings and constructed meanings identified in participants' talk through discourse analysis. This provides initial qualitative support for the argument that the introduction of the Mental Health (Wales) Measure 2010 has served to exacerbate existing service complexity and fragmentation.

In Chapter five, I build upon this picture of service fragmentation and complexity by identifying the theme, 'Competing versions of recovery'. Competing versions of recovery in talk and policy are conceived through distinct instances of colonisation of the concept (Amering and Schmolke, 2009; Barker, 2003; Mind, 2008; Szasz, 1974a; Szasz, 1974b). I also introduce the concept of the 'floating signifier' from the discourse theory of Laclau and Mouffe (1985) which I apply to the concept of recovery.

In Chapter six, I focus on care continuity as the key element in provision of recovery focused services, revealing experiences of care discontinuities which are concentrated at the primary level and its boundary with secondary mental healthcare. I argue that qualitative analysis of these experiences expressed through participants' talk generates evidence of complexity and fragmentation in recovery-oriented primary mental health services in Wales.

In Chapter seven, which is the discussion chapter, I draw together the different threads of my analysis to provide a theoretical explanation of findings. I employ a poststructuralist, complexity theory of discourse to provide a fuller explanation of why complexity is most manifest in discursive practices, positionings and constructed meanings of participants forming misaligned expectations about transforming services. I also develop Laclau and Mouffe's (1985) notion of the floating signifier, applied to recovery in Chapter five, to explain how individuals and groups seek to appropriate the meaning of recovery to suit their interests and agendas. This may lead to a proliferation of competing versions of recovery, which has the potential to contribute to escalating complexity, service fragmentation and discontinuities in mental healthcare. I also apply Jürgen Habermas' theory of colonisation of the lifeworld to 
expand upon the notion of competing recovery versions. This informs an understanding of the Mental Health (Wales) Measure 2010 as a neoliberalist colonisation of recovery, which may be a further factor for service complexity, and may have a distortive effect on the capacity for individuals to construct recovery versions.

I present conclusions of my thesis in Chapter eight, which begins with review of key findings of the study and study limitations. I follow this with a further exploration of Habermasian theories of the ideal speech situation and public sphere (1970a; 1970b; 1991), which are proposed as theoretical solutions to problems of systemic colonisation and form the basis for study recommendations. I then describe the original contribution of the thesis and then finally provide a concluding statement about the challenges facing recovery-implementation. 


\section{Chapter 2: Reviewing the evidence for care continuity and complexity in the provision of recovery-focused mental healthcare}

\subsection{Introduction}

In Chapter one, I set out the initial background and context for my research study, including 'locating myself' reflexively within this study and thesis. This involved a discussion of policy and service context, my own interest in the project, and a reflexive consideration of my orientation and perspective. My aim in this second chapter is fourfold. Firstly I intend to provide further background for the study by review and appraisal of literature relevant to the key concepts of the research inquiry. A survey of the literature indicates gaps in knowledge which point towards appropriate areas for investigation within this study (Bryman, 2016). Secondly, since my research is conceived as a piece of exploratory, qualitative research, examination of relevant subject-areas and theory within the literature is vital to provide a theoretical context for data analysis. It is therefore necessary for me to familiarise myself with relevant literature and theory in order to develop theoretical sensitivity and a theoretical perspective or 'lens' through which I will be able to carry out qualitative analysis of data (Creswell and Plano Clark, 2011; Crotty, 1998; Rivas, 2011; Seale, 2011; Silverman, 2011). Thirdly, the findings of the literature review are intended to provide guidance for key decisions about research design and methodology. In particular, question formation for semi-structured interviews, including prompts and probes, were informed by the evidence synthesis of the literature review (Connell et al., 2014; see Appendices 1 and 2). Finally, the argument which I develop within this literature review forms a rationale underpinning the focus of the overall research inquiry seeking to develop new knowledge about the experiences of people negotiating healthcare systems interfaces. This argument is developed by initially focusing on a review of mental health services impacted by policy, an understanding of which is provided by complex systems theory (section 2.5). Following this, I focus on recovery as the key focus of policy (section 2.6). Lastly, I examine a multi-dimensional conception of care continuity as a key element in the provision of recovery-focused healthcare (section 2.7).

The subject areas I have focused on within the literature review are guided by the research question which articulates the focus of my inquiry. From the research question, "What are the 
experiences of care continuity and recovery for people at the interface of primary and secondary mental health care in Wales?" I drew three main subject areas or domains of inquiry for my literature review. The first of these areas concerned mental health services in Wales and their policy context, which includes the Mental Health (Wales) Measure 2010, and how this may be understood in terms of complex systems theory (Cilliers, 1998; Pickel, 2011; Plsek and Greenhalgh, 2001; Walby, 2007). The other two areas of inquiry for the literature review, which I abstracted from the research question, were recovery and care continuity. These are the theoretical dimensions within which I sought to understand people's experiences of Welsh mental health services. Recovery, which is the second subject area in this literature review, is the focus of contemporary mental health policy since it can be seen as the current, dominant paradigm shaping mental health policy and services (Braslow, 2013; Edgley et al., 2012; Hannigan et al., 2018; Lal, 2010; Morrow, 2013). The recovery paradigm (Bonney and Stickley, 2008; Edgley et al., 2012; Lal, 2010) is successor to previous organising principles of mental health, such as care in the community (Braslow, 2013; Coid, 1994) and state-funded institutions and hospitals (Gilburt et al., 2014). Contemporary mental health policy is now predominantly conceived as recovery-based, and the Mental Health (Wales) Measure can be seen as one example of such a policy-conception (Bonney and Stickley, 2008; Braslow, 2013; Morrow, 2013; Pilgrim and McCranie, 2013; Welsh Government, 2010a; 2013; 2014c). For the third and final area of inquiry in this review, I cover care continuity in the context of mental health services. Good care continuity is widely considered to be a central goal of contemporary mental healthcare (Sweeney et al., 2012). This qualitative inquiry therefore examines care continuity as the key element in provision of complex, recovery-focused healthcare services, as impacted by policy.

In the next section, I describe the search strategy I used to investigate literature and theory in each of the three subject areas.

\subsection{Search strategy}

My choice of search strategy was influenced by my decision to use a scoping study approach for the literature review. A scoping study approach can be used to provide an overview of the literature in multiple subject-areas, and aims to rapidly map key concepts underpinning several 
points of inquiry (Mays et al., 2001; Wiering et al., 2016). The search strategy associated with a scoping study review is often employed when the amount of literature is very large and consequently there are feasibility issues with performing a full-scale systematic review (Davis et al., 2009). Since I decided to investigate three major subject areas, I anticipated that the amount of potential literature to be surveyed would be considerable, and that this would present feasibility issues for my $\mathrm{PhD}$. A scoping study approach therefore seemed to me to be the most sensible way to undertake a literature review with a wide coverage of multiple subject areas.

A scoping study approach can be used to perform an exploratory literature review on multiple fronts to identify key pieces of literature relevant to the research interest (Arksey and O'Malley, 2005). It therefore offers a significant degree of systematisation without being a comprehensively systematic approach. A scoping review is achieved by combining inductive approaches based on database search engines with deductive approaches such as backward chaining from reference lists, and forward citing using online reference gateways such as 'Google Scholar'.

Scoping studies occupy a midway point within a spectrum between the polarities of a narrative review synthesis employing a deductive search strategy, and a tabular, systematic review employing an inductive search strategy (Davis et al., 2009). The following figure represents this spectrum: 


\section{Figure 1: Range of approaches for literature review}

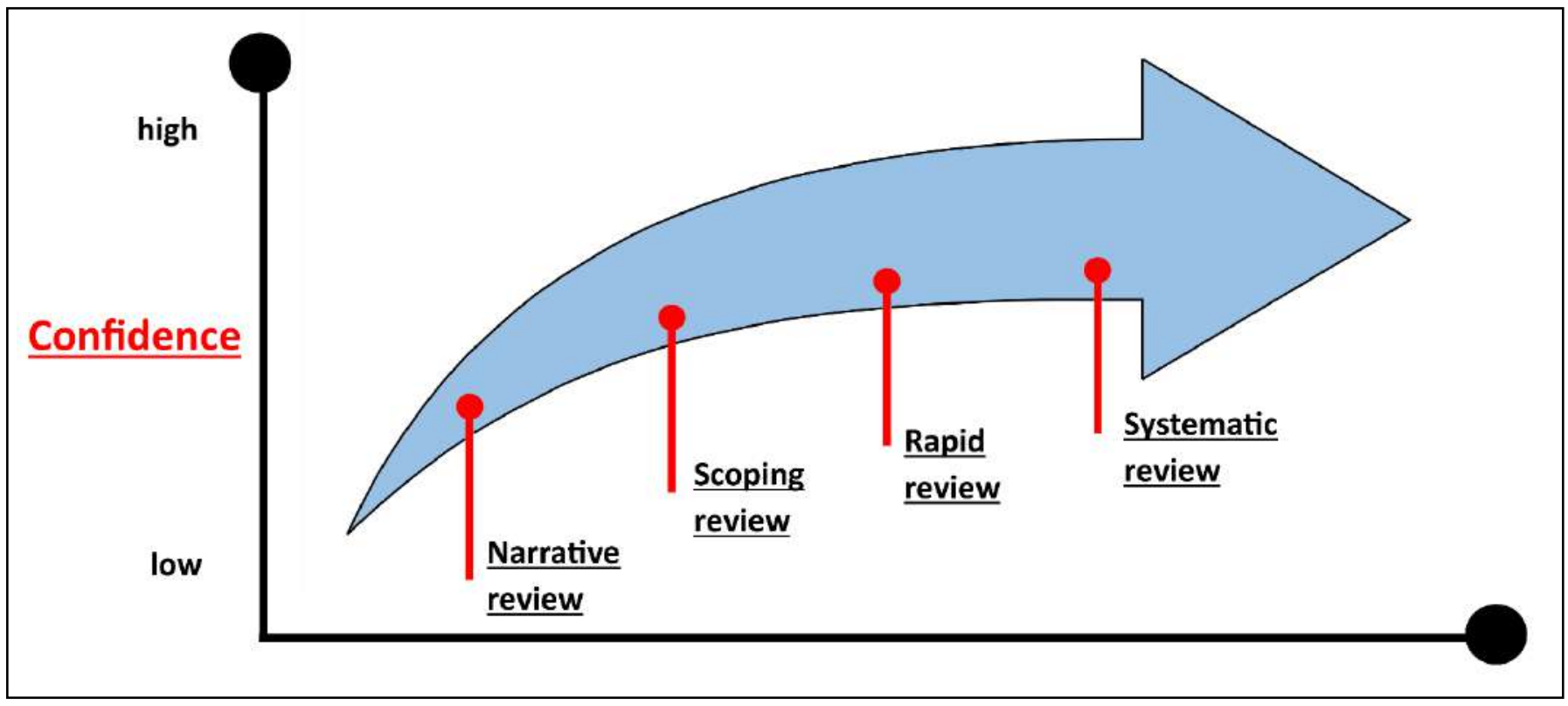

(HLWIKI International, 2016)

In Figure 1, the spectrum ranges between one polarity of highest evidential confidence where a full systematic review is undertaken, and the other polarity of lowest evidential confidence involving a less systematic approach to searching and a narrative synthesis with no tabular accompaniment (Grant and Booth, 2009). The scoping study approach which I employed achieves a compromise between confidence in evidence on the one hand, and scale of literature searching and reviewing on the other, and is therefore placed in the middle of this spectrum, as shown in the above diagram.

My specific scoping review approach was adapted from the methodology described by Arksey and O'Malley (2005). This approach is made up of five stages:

1. Identify the initial research question and determine which aspects of the question are most important to facilitate an understanding to form the basis for inquiry. These aspects are then the subject areas of inquiry for the review (this stage was described in the introduction of this chapter, section 2.1). 
2. Identify relevant studies in each of the study areas in a comprehensive fashion. This was done through a combination of inductive and deductive approaches to literature searching (see following sections 2.2.1 and 2.2.2).

3. Select studies using methods similar to a systematic review but with a more flexible approach involving iterative updating of search strategies and terms as knowledge of the field progresses. I carried out both inductive and deductive searches in this iterative and incremental fashion over the course of the duration of the entire project.

4. 'Charting' of data by synthesising and interpreting publication information through sifting and sorting material according to key issues and themes (Ritchie and Spencer, 1994). See section 2.4 for implementation of this stage.

5. Collate and summarise results using thematic analysis. The level of thematic synthesis does not approach the level of rigour found in a systematic review and may be considered to be closer to the standard of a narrative review (Arksey and O'Malley, 2005). See sections 2.4 to 2.7 where I carry out this stage.

My scoping review also fulfilled the requirements of the 'PRISMA-ScR' checklist for scoping reviews (Tricco et al., 2018). I now describe both inductive and deductive search strategies I employed to identify literature within each of the subject domains.

\subsubsection{Inductive Strategy}

For the inductive search strategy, I used key terms which I inputted into the search engines of bibliographic databases. Terms were generated by the use of a thesaurus and through study of subject-related terminology in initial sets of literature. I employed an iterative approach, where a list of keyword terms were developed and refined as I continued to search the literature throughout the course of the project. I performed inductive searches in the subject domains at the beginning of my project but also kept them up to date with current literature by repeating these searches of bibliographic databases throughout the duration of my project.

I performed a full inductive search in the third area of inquiry, 'Care continuity as a multidimensional concept', described in section 2.7. I considered that this subject area needed to be 
investigated with a high level of systemisation offered by an inductive approach, although this was then supplemented with a deductive search strategy (see section 2.2.2). The reason for this was that care continuity was the key dimension in this study within which I sought to appraise recovery and healthcare experiences at systems interfaces in Welsh services. I published a full systematic review of literature concerning this concept in the context of mental healthcare (Weaver et al., 2017). The review of literature on care continuity presented in section 2.7 is closely related to this publication, though it is more up to date and provides some additional detail on care continuity, including review of a new article by Biringer and associates (2017).

Terms used for inductive searches of bibliographic databases are provided in each of the following sections covering the three areas of inquiry. These terms were combined into Boolean search strings which are provided in Appendix 9. As well as using key terms and Boolean search strings in the various subject areas, I combined these with MeSH (Medical Subject Headings) terms to enhance and focus my searches within the field of mental healthcare and services (Richter and Austin, 2012).

When I performed an inductive search, I entered terms in the form of Boolean strings into the ProQuest portal at https://search-proquest-com.openathens-proxy.swan.ac.uk/. Bibliographic databases accessed through the ProQuest portal were ASSIA, Medline, the British Nursing Database and ProQuest. These databases gave a wide coverage of clinical and sociological publications appropriate for this inquiry. A final search was carried out for the date range 2005 to $21^{\text {st }}$ May 2019, temporally encompassing all previous inductive searches I had conducted at various points throughout the course of the project. I decided on a time-period of 14 years for inductive searches to ensure a wide coverage of recent and older articles. The following additional parameters were also set within the ProQuest interface:

- Peer reviewed journals only

- Exclude duplicate articles

Articles returned were reviewed and discarded if they were not deemed to be relevant to the subject area of inquiry or if they did not fulfil the following inclusion and exclusion criteria: 


\section{Inclusion criteria}

- Articles in the English language only

- Ages 18 to 64 years of age inclusive (this delimits the focus to adult mental health)

- All mental health diagnostic groups and presenting problems either classed as serious mental illness (SMI) or contact with secondary services

- Scholarly articles taken from academic journals

- Published books

- Conference proceedings

\section{$\underline{\text { Exclusion criteria }}$}

- Organic disorders related to mental illness

These inclusion and exclusion criteria were used to delimit the quantity of returned articles without unintentionally eliminating relevant publications. The rationale for these criteria was that literature should be confined to adult mental healthcare discussed in high quality, academic publications written in English. ${ }^{6}$

\subsubsection{Deductive Strategy}

I supplemented inductive searches by also using a deductive strategy for the literature search. It has been noted that inductive search strategies relying on search engines of databases may miss relevant articles (Evans, 2002; Montori, et al., 2004). For this reason, it is recommended that a variety of deductive strategies for searching the literature be used in combination with inductive approaches, such as hand searching through reference lists, author searching, and

\footnotetext{
${ }^{6}$ I have provided this detail here and in Appendix 9 on the inductive search strategy for care continuity so that the reader will be able to replicate my search and returned articles exactly. This should demonstrate to the reader the rigour and scientific validity with which I carried out this systematic part of the review.
} 
searching forward citations within Google Scholar (Aveyard, 2007). I used all of these techniques for my scoping review search strategy to broaden the body of literature beyond those merely identified by the inductive search strategy. This also allowed me to include a greater variety of types of literature within this review, including journal articles, conference proceedings, grey literature and published books. Greenhalgh and Peacock (2005) describe a process called 'snowball sampling', which I employed, whereby the search strategy evolves adaptively and iteratively in response to reflection upon new knowledge generated by the literature review. I also identified seminal and influential papers where these were located in reference lists of multiple publications, utilising an approach described in O'Connor's 'Network Theory' (1992). This included some publications published prior to 2005, thus broadening my search beyond the limits of the inductive strategy. Finally, I made continued use of university library search engines and the physical library resource for identifying relevant published books, which were not necessarily listed within electronic bibliographical databases.

\subsection{Quality appraisal}

Throughout the process of researching relevant literature, I was attentive to issues of publication quality and validity. This perspective was important for me to determine the credibility of findings, claims and theoretical assertions made within studies. In the course of writing my paper on concepts, models and scales of care continuity (Weaver et al., 2017), I adapted CASP quality appraisal tools for literature reviews, qualitative studies, quantitative studies and cohort studies (CASP UK: Critical Appraisal Skills Programme, 2013; see https://casp-uk.net/) to create a combined, universal quality assessment tool. I then used this as a single tool to appraise the quality of various types of published articles. The tool is shown below in Table 1. 


\section{Table 1: Universal quality assessment tool}

\begin{tabular}{|l|l|}
\hline Category & Score: Weak, \\
\hline Loderate, Strong \\
\hline Citerature review $\&$ appropriate aims, objectives, research design, methodology & \\
\hline Ethics & \\
\hline Clear data collection \& analysis & \\
\hline Good recruitment strategy & \\
\hline Rigour of analysis (qualitative only) & \\
\hline Quality of literature search (literature review only) & \\
\hline Quality of literature synthesis (literature review only) & \\
\hline Quality appraisal for literature (literature review only) & \\
\hline Selection and sample bias elimination; blinding (quantitative only) & \\
\hline Confounding factors identified? (quantitative only) & \\
\hline Result precision (quantitative only) & \\
\hline Generalisability (sample size, limitations) & \\
\hline $\begin{array}{l}\text { Psychometric validation (measurement scale only) } \rightarrow \text { based on } \\
\text { COSMIN checklist }\end{array}$ & \\
\hline Follow up long enough \& low attrition (cohort / longitudinal only) & \\
\hline Discussion \& conclusion quality & \\
\hline Clear statement of findings & \\
\hline Impact, recommendations and further research & \\
\hline
\end{tabular}

I gave an overall quality rating to articles using an approach based on a procedure adapted from the 'Effective Public Health Practice Project' (Thomas et al., 2004). I describe this as follows. Each field applicable to the type of study was rated on a three-point scale of strong, moderate or weak quality. Where the field was not applicable to the type of study, I would leave the field blank. Articles with no more than one weak rating and at least a third strong ratings were considered strong. Articles with less than a third strong ratings and no more than one weak were considered moderate. Finally, articles with two or more weak ratings were considered weak.

Using this method, I was able to provide an evaluation of all articles systematically searched for within the subject area of care continuity. I confined comprehensive use of the tool to this 
subject domain because of the feasibility issues which would have ensued if it had been used systematically in all subject domains of the review. In the other subject areas, I instead used my universal quality assessment tool as a general guide for screening and appraisal.

It should be noted that the assessment tool contains one field related to psychometric validation. This field is used where the study describes psychometric validation of measurement scales for care continuity. Appraisal of the methodological quality of psychometric validation is based on the COSMIN checklist ('Consensus-based Standards for the selection of health status Measurement Instruments'; Mokkink et al., 2012; Uijen et al., 2012). This represents a consensus evaluation for the methodological quality of psychometric validation applied to measurement tools. I used this consensus evaluation as the basis for appraisal within the field related to psychometric validation where this was applicable.

\subsection{Evidence Synthesis}

I used a thematic synthesis approach employing the presentational style of a narrative review (Barnett-Page and Thomas, 2009; Lucas et al., 2007), in accordance with the fourth and fifth stages of Arksey and O'Malley's (2005) scoping study approach. This enabled me to identify various themes contextually within each subject domain. This approach involved reading and re-reading publications identified by the search strategy so that I gained a full understanding of argumentation and study design. Use of the universal quality appraisal tool adapted from CASP frameworks assisted me in this activity. Topics and relevant sections of information were labelled and coded using the 'Adobe Acrobat' comments tool. This was then combined with extensive use of the 'Endnote' application to organise and navigate papers and the notes contained within them. I then used concept mapping software, called 'Compendium' (http://compendium.open.ac.uk/), to organise these topics distributed throughout multiple publications as the basis for constructing themes. These themes provide the underlying structure for the narrative review which I present in sections 2.5 to 2.7.

I now present findings of the literature review, starting with the subject area, 'Complexity theory, mental health services and the impact of policy'. 


\subsection{Complexity theory, mental health services and the impact of policy}

In this section, I describe my investigations of literature about modern healthcare systems, the effects of recent policy trends, and a theoretical understanding of these phenomena grounded in complexity theory. The following search terms were used for inductive searches of the subject domain covering complex systems in mental health care and services:

\{systems theory; complex systems; complexity theory; wicked problem; waves of consequences; cybernetics; autopoiesis; social systems

Some specific terms such as 'wicked problem' and 'wave of consequence' were sourced in publications which I had come across prior to conducting the review, linking contemporary mental healthcare to complex systems theory (Hannigan and Coffey, 2011; Hannigan, 2013). Other more generic terms were found in introductory text books and chapters on complex systems theory (Byrne, 1998; Cillers, 1998; Hooker, 2011; Pickel, 2011). My approach of thematic evidence synthesis in this subject area led to the generation of two themes:

- Complexity theory and mental healthcare systems

- The impact of policy on mental health systems complexity

I commence with a review of literature on complexity theory and how it may apply to modern mental healthcare systems. This is then followed by a review of literature discussing how successive policy programmes have impacted services in such a way as to escalate this complexity. Note that the studies covered in this subject area under review are predominantly discussion papers and not research studies. Consequently, there is little information on study design, methodology or sampling in this section.

\subsubsection{Complexity theory and mental healthcare systems}

Three types of early systems theory in social science may be identified in reviewed literature, which form the background for complex systems theory (Pickel, 2011). The first type is a historicist conception of social systems such as Marx's historical materialism (Croce, 1914), 
Comte's positivism and Spencer's evolutionary historicism (Harrington, 2005). The second type concerns structuralist-functionalist conceptions of social systems such as Levi-Strauss's structuralism (Hodge and Kress, 1988), Durkheim's social realism (1982 [1938]), and Talcott Parson's system theories (1951a; 1961). The third type are action-orientated theories such as neoclassical economics (Pickel, 2011). However, within sociology, early systems theory has been widely discredited for its linear and reductionist nature, and been replaced by complex systems theories (Walby, 2007). Complex systems theory has been developed into various forms of social theories and comprehensive philosophical frameworks for describing the world and society (Forrester, 1968; Luhman, 1995; 2012a; 2012b; Maturana and Varela, 1980; Walby, 2007; Wiener, 1948). The most all-encompassing of these is the social systems theory of Niklas Luhmann (2012a; 2012b) who views complexity as the central theoretical concern in the social scientific endeavour of late modernity (Holub, 1991). His systemic theory incorporates other comprehensive complex systems theories such as cybernetics (Forrester, 1968; Wiener, 1948) and autopoiesis (Maturana and Varela, 1980; von Foerster, 1962; von Foerster and Zopf, 1981). Cybernetics systems theory is employed by Luhmann to model complexity, after the influence of Parsons (1951a; 1961). In his structural-functionalism, Talcott Parson's employs a cybernetic systems model which tends towards stability and equilibrium (Craib, 1992). Equilibrium is maintained through feedback loops based on the 'AGIL' paradigm ${ }^{7}$ distributed throughout the entire system (McClelland and Thomas, 2006; Parsons, 1951a). Luhmann employs cybernetics in a different way to Parsons to model a system which is complex and may tend towards disequilibrium. Additional dimensions of reflexivity and differentiation are introduced into his systems theory (Holub, 1991; Luhmann, 1995, Osterberg, 2000). These factors have the potential to preserve or even increase complexity though positive feedback loops (Cilliers, 1998, Holub, 1991; Segre, 2014). A complex cybernetic systems model articulated in this way by Luhmann therefore exemplifies the ontological nature of complex systems theory.

Complex systems theory has had a wide application for modern healthcare systems Greenhalgh et al., 2010; Hannigan, 2013; Hannigan and Coffey, 2011; Plsek and Greenhalgh, 2001). In

\footnotetext{
${ }^{7}$ Parson's theory stipulates that every system must satisfy four functional prerequisites for its continued survival. It must adapt (A) to its external environment, attain goals (G), achieve systemic integration (I) and reproduce latent cultural patterns (L) (Crossley, 2005; Parsons, 1951a).
} 
their analysis of modern healthcare services as complex systems, Plsek and Greenhalgh (2001) assert that the limitations of reductionist thinking and the 'clockwork universe' metaphor can no longer capture the niceties of complex adaptive systems such as healthcare services. Complex systems are inherently non-linear and unpredictable, self-organising or autopoietic, which is seen as a more accurate description of the behaviour of modern healthcare systems (Greenhalgh et al., 2010; Plsek and Greenhalgh, 2001).

Hannigan and Coffey (2011) describe modern mental healthcare services also as a complex system and employ the concept of a 'wicked' problem (Rittel and Webber, 1973) to provide a way of understanding current challenges facing services, which they describe as follows:

"We are calling them 'wicked' not because these properties are themselves ethically deplorable. We use the term 'wicked' in a meaning akin to that of 'malignant' (in contrast to 'benign') or 'vicious' (like a circle) or 'tricky' (like a leprechaun) or 'aggressive' (like a lion, in contrast to the docility of a lamb)."

(Rittel and Webber, 1973, p.160)

With wicked problems, problems and solutions are inextricably linked so that there is an inherent systemic circularity (Hannigan and Coffey, 2011). Here, the key dynamics generating a phenomenon are themselves a function of the conditions emerging from the same phenomenon (Hooker, 2011). The result of this is that the effect of any activity can feed back onto itself, potentially creating a positive feedback loop. The discovery of positive feedback loops in complex systems theory (Walby, 2007) challenged the notion within traditional systems theory that a system would always return to a state of equilibrium through the operation of negative feedback loops (Durkheim, 1982 [1938]; Parsons, 1951a). Given the possibility of positive feedback loops, relatively small changes in mental health service systems may generate "waves of consequences" (Rittel and Webber, 1973, p.163) which are both unpredictable and far outweigh the initial factors, thus moving the system far from a state of equilibrium (Cilliers, 1998; Plsek and Greenhalgh, 2001). The key features of complex systems, such as positive feedback loops, non-linearity, autopoiesis and wicked problems, have therefore been highlighted as characteristic of contemporary mental health services. An underlying perspective of this thesis is therefore an understanding of mental health services as a complex system, exhibiting many of these features. 


\subsubsection{The impact of policy on mental health systems complexity}

Pressman and Wildavsky (1973) argue that relatively small failures of cooperation between different elements can multiply in such a way as to engender positive feedback loops, which are characteristic of complex systems. This is particularly the case when governments attempt to enforce change through successive top-down approaches, which has increasingly occured in the recent history of UK mental health services (Chapman, 2004; Gilburt et al., 2014; Gilburt, 2015; Glasby and Tew, 2015). Consequently, service developments based on central control and top-down approaches such as the Mental Health (Wales) Measure 2010 may produce unexpected and even counter-productive results in accordance with the predictions of complex systems theory (Hannigan, 2013; Plsek and Greenhalgh, 2001; Pressman and Wildavsky, 1973; RCPsych, 2014). Such an effect was described in section 1.2 where increased care planning rigour under Part 2 of the Measure has given rise to a higher level of discharges to primary care than was anticipated (Gofal, 2014; 2015; ORS, 2014). The process described in section 1.2 can be seen as an unforeseen consequence of the Mental Health (Wales) Measure 2010. This may be understood as a complex systems effect, or more precisely, as a 'wave of consequence' (Hannigan and Coffey, 2011; Rittel and Webber, 1973), which has emanated from the original 'wicked problem' inherent in the challenge of delivering recovery-based services.

A key factor in this and similar processes of escalating complexity is the shifting of professional roles and responsibilities for the psychiatrist and CMHTs. The tensions this shift creates in professional groups has been highlighted by Hannigan and Coffey (2011), occurring in the context of a constantly changing and increasingly fragmented mental health system (Gilburt et al., 2014; Gilburt, 2015; RCPsych, 2014). Micro-level phenomena such as human agency, work and responsibilities may interact with macro-level phenomena such as policies to produce unexpected and uncontrollable outcomes in the manner of a complex system (Greenhalgh and Plsek, 2010; Hannigan, 2013).

Major policy changes such as the Mental Health (Wales) Measure 2010 arguably give rise to increased complexity in an already complex mental health system. This could be due to difficulties in implementation (RCPsych, 2014), or an unintended consequence of increasing barriers between sectors of mental health services (WaMH in PC, 2015). Additionally, as 
policy-makers attempt to enforce change through a "torrent of top-down actions" (Hannigan and Coffey, 2011, p.220), this is likely to provoke resistance from vested interests and generate further unintended consequences (Glasby and Tew, 2015; Rogers, 2003). Hannigan and Coffey (2011) note:

"Particularly complex divisions of work have evolved, with services being provided by an array of statutory and non-statutory workers, lay carers and others, situated in both community and institutional settings. Roles and responsibilities have shifted, sometimes in response to policy change and organisational pressures, and from time to time disputes over professional boundaries have broken out. Distinctively, in the mental health field disagreement over theory and practice continues, in ways which cross-cut occupational demarcations."

(Hannigan and Coffey, 2011, p.222)

The current shift towards an emphasis on primary care taking the lead in mental health provision as opposed to its more traditional gatekeeper role, and the difficulties with providing care coordinators for those on psychiatrists' lists (see section 1.2) may be seen as an example of a shift in professional roles, which can be problematic for key groups within mental health services (RCPsych, 2014). This may in turn generate discontinuities of care, increased complexity at systems interfaces and other unpredictable effects which are typical of complex systems (RCPsych, 2014).

Belling and colleagues (2011) examined the impact which blurring of professional roles and jurisdictional claims has had upon care continuity and coordination. They conducted a qualitative study involving semi-structured interviews within a random sample of health and social care professionals $(n=113)$, combined with the use of a structured questionnaire. In so doing, they found that confusion over professional role boundaries, lack of support for training and role development, and workforce levels and workloads are all implicated as factors behind discontinuities of care, and disruption of care coordination (Belling et al., 2011). In a similar way, as service provision has become ever more complex, transformation of traditional care pathways engendered by the Measure (RCPsych, 2014) may have the effect of disrupting patterns of care continuity and care coordination within contemporary Welsh services.

In sum, an increasingly complex and fragmented mental health system in the UK and Wales responding to top-down legislation attempting to solve 'wicked problems' may lead to 'waves 
of consequences' in terms of individual roles and responsibilities, negotiation of service interfaces, and overall systemic complexity. In the light of this perspective, policies such as the Mental Health (Wales) Measure 2010 may be seen to have had the effect of escalating complexity in mental health services in Wales.

In the next section, I focus my review upon recovery as the key focus of policy. Having reviewed literature to identify the role of policy in escalating complexity in mental health services, I now examine the focus of contemporary policy on recovery approaches.

\subsection{Recovery as the focus of contemporary mental health policy}

This section covers my review of the recovery concept and its implementation as policy within the contemporary mental health field. I will focus first on the recovery concept and its history, and then on its implementation in the form of policy.

One of the immediate issues I encountered when studying recovery was the great variety of ideas, theories and approaches concerning the concept. As an illustration, an inductive search of recovery, using the terms presented below, with the same parameters and databases as the search on care continuity yielded $n=24,635$ articles!

To focus the search for my review, I looked first at literature reviews and book publications attempting to provide a comprehensive assessment of recovery models, theories and concepts. Bonney and Stickley (2008), in their review of over 170 publications, identify six dominant themes within various recovery theories and approaches, which are social inclusion, establishing service users' identity, the influence of recovery theories on services, empowerment, hope, and risk management. Leamy and colleagues (2011) establish a conceptual framework for recovery on the basis of a systemic review of $n=97$ papers and a modified narrative synthesis approach (Jabareen, 2009). This conceptual framework is made up of the components of social connectedness, hope and optimism about the future, establishing identity, finding meaning in life, and empowerment. Pilgrim and McCranie (2013), in their published book providing a critical sociological account of recovery, propose four different shades of meaning for recovery: personal journey, a critique of services, therapeutic optimism 
and a social model of disability. The latter meaning in Pilgrim and McCranie's (2013) survey finds analogy between approaches to recovery and the social model of disability where there is a focus on strengths rather than deficits in individuals (Barry and Yuill, 2016; Nettleton, 2013). A recently formulated health measurement scale, 'Recovering Quality of Life' or 'ReQoL' (Keetharuth et al., 2018) uses rigorous qualitative and statistical procedures to create a Patient Reported Outcome Measure (PROM) (Black, 2013) for measurement of mental health recovery in seven domains: wellbeing and 'illbeing', physical health, daily activity, hope and hopelessness, self-perception, autonomy, and relationships (Connell et al., 2012).

Reflecting on the literature in this initial survey, it became clear to me that there was a lack of consensus about the nature of recovery, or even agreement about the specific core features which could be attributed to the concept. I considered that this diversity might be a potential factor behind the proliferation of versions of recovery. Additionally, the recovery concept is controversial in some circles, due its contested nature and difficulties in clarifying the precise nature of its underpinning values (Bonney and Stickley; Glasby and Tew, 2015; Mind, 2008; see also https://recoveryinthebin.org/). I therefore decided to focus on the contested nature of the concept in my review, in order to develop a critical account of recovery.

I employed the following terms in various inductive searches conducted over the course of the project. These terms were entered into search engines using various combinations:

\{recovery; empowerment; recovery star; WRAP; wellness and recovery action plan; wellness recovery action plan; tidal model; social inclusion; personal journey; critique of services\}

The terms were generated from initial textbook literature on recovery which I read during the early stages of this project (Amering and Schmolke, 2009; Pilgrim and McCranie, 2013; Repper and Perkins, 2003; Slade, 2009). I developed an additional set of terms to refine the search to focus on the contested nature of the concept, and related issues of appropriation of the concept (Barker, 2003; Mind, 2008; Pilgrim, 2008):

\{concept; model; theory; colonisation; tokenisation; co-opt\} 
Full Boolean search strings based on these terms may be found in Appendix 9. Thematic synthesis then generated the following three themes:

- The rise to prominence of recovery

- Recovery, a 'polyvalent' concept

- Recovery as a neoliberalist policy

I now provide a narrative account within each of these three themes.

\subsubsection{The rise to prominence of recovery}

Originating with service users in the 1980s and 1990s (Anthony, 1993; Deegan, 1988; Deegan, 1993), and in the USA (Bonney and Stickley, 2008; Braslow, 2013), the concept of recovery has grown into one of the most important paradigms and prominent rhetorics within modern mental health services (Pilgrim and McCranie, 2013; Woods et al., 2019). Recovery continues to be highly influential in contemporary mental health services at both national and international levels. It occupies the forefront of policy agendas (Bonney and Stickley, 2008; Braslow, 2013; Morrow, 2013; Pilgrim and McCranie, 2013; Welsh Government, 2014c), and is now considered to be the contemporary paradigm for mental health services and policy implementation (Braslow, 2013; Edgley et al., 2012; Hannigan et al., 2018; Lal, 2010). The World Health Organisation report on primary mental healthcare integration places a selfmanagement recovery approach at the foundation of the WHO service pyramid, on which all care is based (WHO, 2008). Policy implementation of recovery-based services has become increasingly prevalent in a variety of countries including the USA, Canada, New Zealand and the UK (Gilburt, 2013; SAMHSA, 2004). Additionally, the pre-eminent role of the care programme approach (CPA) in England and the use of the Care and Treatment Plan (CTP) for care coordination and continuity in Wales is now underpinned by a commitment to a recoveryethos (Hall et al., 2013; Hannigan et al., 2018; Simpson et al., 2016b; Welsh Government, 2014c; 2015; 2016). 


\subsubsection{Recovery a 'polyvalent' concept}

Despite the prominence of a recovery paradigm in contemporary mental health services, it has been called a 'polyvalent' concept (Pilgrim, 2008), highlighting the difficulties and lack of consensus surrounding its meaning, application and implementation (Davidson et al., 2005; Lal, 2010; Leamy et al., 2011). In their review of the literature, Bonney and Stickley (2008) note that there is no universal definition of recovery and that it has a multitude of meanings, being "an idea, a movement, a philosophy, a set of values, a paradigm, policy and a doctrine for change" (p.140). Conceptualising recovery defies experts in the mental health field and has split opinion on the matter (Bonney and Stickley, 2008; Lal, 2010). Some see it as a simplistic and vague concept, and a "co-opted, near-toothless gospel of hope" (Hopper, 2007, p.877). On the other hand, others see it as a revolutionary, emancipatory paradigm within modern mental healthcare (Anthony, 1993; Bonney and Stickley, 2008; Deegan, 1988; Edgley et al., 2012; Repper and Perkins, 2003). This has led to the view that a lack of clarity about the meaning of recovery serves as a rallying point for different groups with different agendas (Pilgrim and McCranie, 2013). These groups have adopted different shades of meaning for recovery in order to serve the purposes of their disparate intentions, determined by differing contexts and circumstances (Davidson et al., 2005; Jacobson and Greenley, 2001). Despite this, it has been commented that the diversity of meanings appropriated for recovery may be seen as a source of strength for the concept, rather than there being a need to provide a unitary definition for the concept (Hopper, 2007; 2008; Mind, 2008).

A consequence of the contested nature of the recovery concept is the potential for different people to assume that they are talking about the same thing when they employ the term (Hopper, 2007; 2008; Pilgrim and McCranie, 2013). Recovery has the potential to mean different things to different individuals (Davidson et al., 2005; Simpson et al., 2016a), or even all things to all people (Edgley et al., 2012). For example, the mental health user / survivor movement emphasises self-management skills and strengths over a deficits model grounded in mental health pathology, in line with the social model of disability understanding explained by Pigrim and McCranie (2013). Under this conception, recovery is understood as "living with and growing beyond" (Repper and Perkins, 2003, p.45) mental illness, and an emancipation from coercive and iatrogenic services (Edgley et al., 2012; O'Keeffe et al., 2018; Pilgrim 2008; 
Pilgrim and McCranie, 2013; Repper and Perkins, 2003; Tew et al., 2011). Conversely, policy implementations of recovery emphasise reduction in service dependency and service provision (Ramanuj et al., 2015), which is viewed by some as a veiled attempt to ration and dilute services (Dickerson, 2006; Jacobson and Greenley 2001; McKeown et al., 2017; Mind, 2008; Robertson, 2016; Slade et al., 2014). Consequent to this last interpretation of recovery, some activists, such as 'Recovery in the Bin', have rejected the concept altogether (Harper and McKeown, 2018; see https://recoveryinthebin.org/). According to this group, recovery is a fundamentally flawed notion, whose imprecise meaning renders it too easily co-opted by a neoliberalist agenda. They view this neoliberalist co-option as a cover for coercion, victim blaming and removal of services under a drive towards austerity. The alternative term 'unrecovered' is adopted by this group to express their dissension towards neoliberalist recovery (Harper and McKeown, 2018). This latter view is one instance of the broad observation about the colonised status of recovery models (Amering and Schmolke, 2009; Barker, 2003; Mind, 2008). This view, that a polyvalent recovery concept is vulnerable to invasion by other theories and agendas, is an application of the original notion of 'psychiatric colonisation' introduced by Thomas Szasz (1974a; 1974b) within the radical antipsychiatry critique.

\subsubsection{Recovery as neoliberalist policy}

A neoliberalist consensus on recovery has developed to the point that it has become enshrined in mental health policy frameworks (Department of Health and Human Sciences, 2003; DoH, 2009; SAMHSA, 2004; Gilburt, 2013). In recent years, recovery has become the dominant ideology and organising principle of mental health services (Braslow, 2013; Pilgrim and McCranie 2013; Woods 2019). In a way which has mirrored neoliberal thinking on welfare reform and poverty, recovery has been seen as representing a way to liberate people from dependency on systems of care (Braslow, 2013). Whilst this neoliberal association is seen by groups such as 'Recovery in the Bin' as indigenous to a dubious concept which should be abandoned, others see recovery as essentially benign, but having been appropriated by neoliberalist politics in a way which distorts its true meaning (Edgley, 2012; Perkins and Repper, 2018; Rose, 2014; Thomas, 2016). More recently, this distorted version of recovery 
has been labelled 'neorecovery' by the 'Recovery in the Bin' group (Recovery in the Bin, 2019).

The Mental Health (Wales) Measure 2010 may be regarded as one such instance of policy because of its emphasis on self-sufficiency within the context of primary care (Lester and Gask 2006; Pilgrim and McCranie, 2013; Ramanuj et al., 2015). An ethos of self-management-based recovery, governed by prudent healthcare principles (Aylward et al., 2013), is embodied in a drive under the Measure to create services which maximise independence and promote empowerment and choice (Welsh Government, 2015). Consequently, "discharge from specialist care (such as secondary mental health services) is regarded as a key outcome of the recovery model within mental health" (Welsh Government, 2010a, p.42; 2013, p.11; 2014c, p.15). The Measure emphasises self-management and independence though increased treatment within primary care, alongside reduced dependency upon secondary services. Arguably, it achieves not only the goals of a particular brand of recovery model, but also accomplishes the purposes of financially motivated agendas stemming from implicit neoliberalist ideologies governing the actions of policy-makers (Braslow, 2013; Morrow, 2013; Woods et al., 2019). These agendas include cost-cutting and a drive towards achieving greater efficiency, which may be identified within prudent healthcare principles (Aylward et al., 2013). The Measure can therefore be situated within the neoliberal consensus of certain policy-makers on recovery, and can be seen as an instance of this kind of colonised recovery version (Department of Health and Human Sciences, 2003; DoH, 2009; SAMHSA, 2004).

However, this and other similar types of policy-implemented recovery, driven by financial pressures and focused on a return to primary care, have been criticised as a misinterpretation or even a distortion of the recovery model (Lester and Gask, 2006; Ramanuj et al., 2015; Recovery in the Bin, 2019; Slade et al., 2014; Woods et al., 2019). It is argued that such versions of recovery are heavily influenced by the introduction or colonisation of external agendas like policy objectives to cut welfare, and employment schemes to get people back to work such as 'Pathways to Work' (Becker et al., 2010; Braslow, 2014; Mind, 2008; Morrow, 2013). This conception could feed into the radical critique of the 'Recovery in the Bin' movement, which in this respect is applicable to the Mental Health (Wales) Measure 2010. 
I now move on to my review of care continuity, which is conceived as the key element in the provision of recovery-focused, complex healthcare systems, impacted by policy. Care continuity was conceived as a key element given the study focus on movement of people between different parts of the mental healthcare system in Wales. Care continuity therefore stands as a concern very logically related to this inquiry, and indeed it is essential that it is examined by review as a basis for this qualitative study.

\subsection{Care continuity as a multi-dimensional concept}

A full, systematic review of concepts, models and scales of care continuity is presented in my published journal article (Weaver et al., 2017). This section partly draws on this work. I carried out an additional search of literature to update the review prior to submitting this thesis. This more recent search yielded an additional paper to those reviewed in my previously publication, a qualitative study of various aspects care continuity by Biringer and associates (2017). This study was of particular interest for this review since it relates a multi-dimensional appraisal of care continuity experiences to recovery. This connection is also made by other studies in this review (Poremski et al., 2016; Sweeney et al., 2012; Uijen et al., 2014), supporting my argument in this review that care continuity is a key element in delivery and experiences of recovery-focused services.

To perform the part of the review, I used the following terms for the literature search:

\{care continuity; continuity of care; care coordination; case management; care planning\}

These terms were based on those used in my published systematic review on care continuity (Weaver et al., 2017). A Boolean search sting was constructed out of these terms and is shown 
in Appendix 9. My updated search yielded one additional paper by Biringer and colleagues (2017), which had been published after my original systematic review.

Figure 2 below shows a flow diagram of the inductive search for this part of the literature review on care continuity.

Figure 2: Search strategy flow chart for care continuity

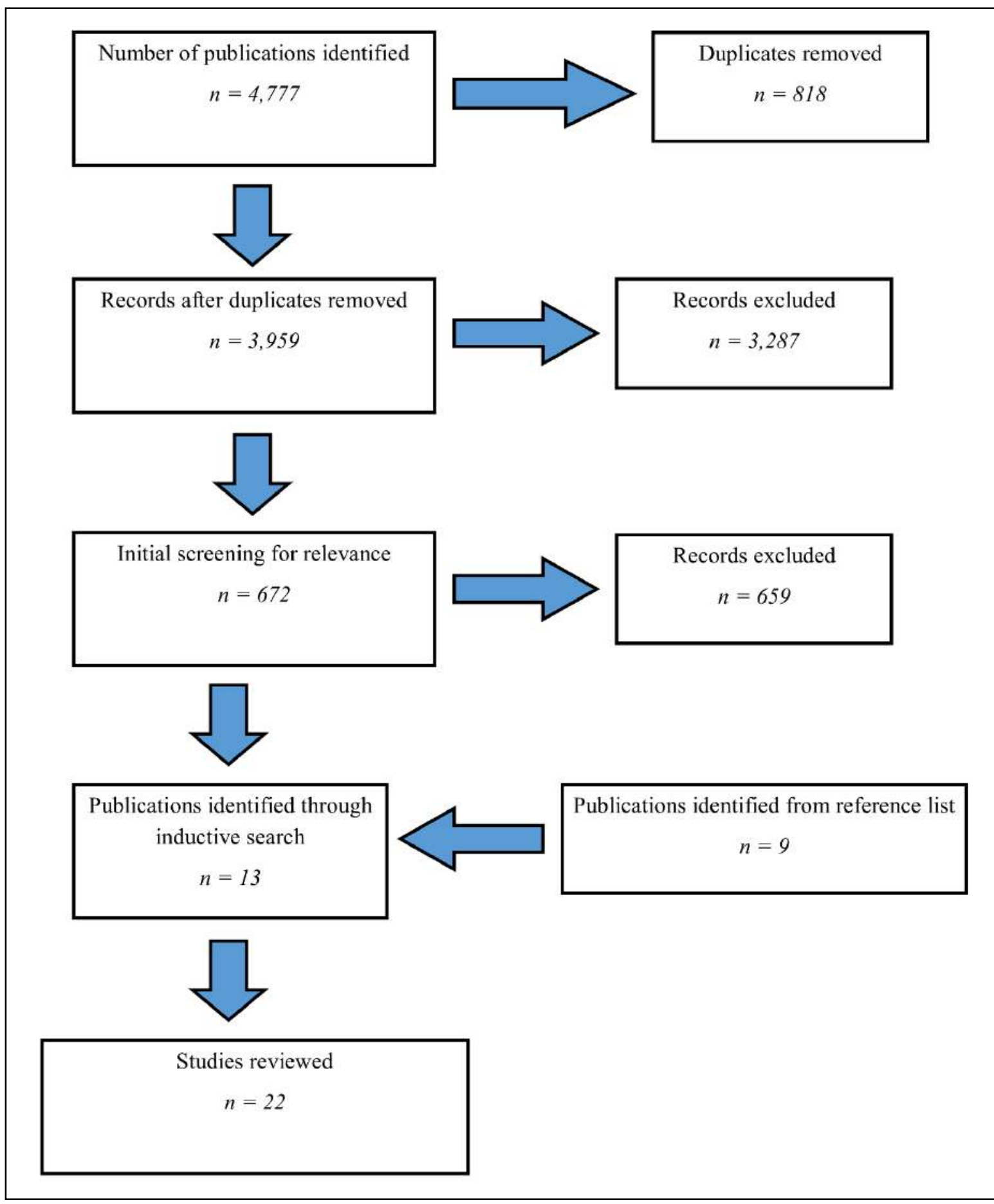


It can be seen in this figure that initially $n=4,777$ (on 21/05/2019) articles were returned by the search on care continuity and care coordination papers, before duplicate removal. After duplicate removal, $n=3,959$ articles remained. These were then manually screened for relevance to a theoretical understanding of care continuity so that $n=672$ remained for the subject domain. Articles about care continuity were screened a second time in terms of whether or not they involved a multi-dimensional conception of care continuity, after which $n=13$ articles remained. The rationale for using this focus on multi-dimensional concepts of care continuity to refine the search strategy was that, in the light of the contested nature of this fundamental concept in healthcare, it has been increasingly conceived as a multidimensional or 'multi-axial' concept (Biringer et al., 2017; Freeman 2000; 2002; Joyce et al., 2004; Puntis et al., 2014). Consequently, unidimensional conceptualisations of care continuity are now considered to be inadequate (Joyce et al., 2004). A major support for a multi-dimensional conceptualisation has been its confirmation by factor analysis by Burns and colleagues (2009). In my systematic review (Weaver et al., 2017), I concluded that articles supporting a multidimensional framework for care continuity provide the most relevant understanding of the concept. I therefore undertook a review of literature of care continuity relevant to and providing further support for this understanding, to form the basis for my qualitative inquiry.

My inductive search strategy was supplemented with a deductive approach (see section 2.2.2) to identify further papers relevant to a multi-dimensional theoretical conception of care continuity. A further $n=9$ papers were identified in this way bringing the final tally of papers for this section of the review to $n=22$. My thematic synthesis approach produced the following seven themes:

- Theoretical introduction to care continuity as a multi-dimensional concept

- Care continuity measurement scales

- Experienced continuity

- Relational continuity

- Longitudinal continuity 
- Cross-boundary continuity and care coordination

- Informational continuity

The first of these themes is an introduction to the theoretical, multi-dimensional concept of care continuity. I follow this with a theme concerning mental health care continuity measurement scales which provide psychometric support for a theoretical understanding of care continuity as a multi-dimensional concept. This is followed by five themes concerning key dimensions of care continuity identified in the reviewed literature. I will now provide narrative review under each of these themes. A table summary of the review is provided in the appendices (Table 6 in Appendix 11).

\subsubsection{Theoretical introduction to care continuity as a multi-dimensional concept}

Continuity of care is touted as a cornerstone of modern health care and considered to be critical to achieving effective treatment (Biringer et al., 2017; Burns et al., 2009; Puntis et al., 2014; Sweeney et al., 2012). It is widely considered to be a central goal of contemporary mental health care (Sweeney et al., 2012), and has displaced previous central concepts of care such as dependency and deinstitutionalisation (Burns, et al., 2009; Ware et al., 2003). Continuity of care has been defined as "the long-term delivery of care that is coordinated among services and is appropriate to a patient's current needs" (Puntis et al., 2014, p.1).

A multi-dimensional construct for care continuity was first fully described by Freeman and colleagues using a multi-axial model $(2000 ; 2002)$. This large project, involving systematic review, four field studies and a Delphi-study, specified six generic dimensions and a further two mental health related dimensions for continuity of care. The two dimensions of longitudinal and cross-boundary continuity are core structural features of this multidimensional model (Ware, et al., 2003). Longitudinal continuity refers to continuous and uninterrupted care over time, whereas cross-boundary or cross-sectional continuity refers to the importance of there being links between services, professionals and components of care (Freeman, 2002). This tally of dimensional features has been added to by subsequent researchers to include areas such as care transitioning, staff availability and crisis transitioning (Burns et al., 2009; Freeman, 2002; Schultz and McDonald, 2014; Uijen et al., 2012). Each 
dimensional concept has also been refined and enriched as various models and measures of care continuity have been developed by researchers within the field (see for instance: Adair et al., 2003; Belling et al., 2011; Crawford et al., 2004; Rose et al., 2009; Ware et al., 2003). A major contribution to this theoretical understanding has been made in the development of measurement scales of care continuity, which I review in the next section.

\subsubsection{Care continuity measurement scales}

I reviewed studies relevant to measurement scales which are structured according to a multidimensional understanding of the care continuity concept. Although I make no direct use of measurement scales in my inquiry, studies associated with development of scales were included in this (and my previously published) review as key contributors to the multi-dimensional conception of care continuity. Since these were developed with standards of psychometric rigour and quantitative testing (Streiner et al., 2015), reviewed scales provide strong scientific support for the idea of care continuity as a multi-dimensional concept, which is one of the key conceptual bases for this inquiry.

The principal scales for care continuity identified by the literature search are 'CONTINU-UM' ('CONTINUity of care-User Measure'), 'CONNECT' (note this is a name and not an acronym) and the Alberta Continuity of Services Scale-Mental Health (ACSS-MH) (Adair et al., 2005; Durbin et al., 2004; Joyce et al., 2004, 2010; Rose et al., 2009; Ware et al., 2003). Out of these three scales, studies testing psychometric properties of the 'ACSS-MH' received the best ratings according to the 'COSMIN' checklist for methodological quality (Adair et al., 2005; Durbin et al., 2004; Joyce et al., 2010; Uijen et al., 2012). The methodological quality of the development of the ACSS-MH scale is associated with an extensive and complex factor structure, described in a number of associated studies (Adair et al., 2005; Durbin et al., 2004; Joyce et al., 2010). This fed into the field for psychometric methodological quality in the assessment tool with the consequence that none of these studies received a weak overall rating, whilst the studies by Joyce and associates (2004; 2010), examining individual dimensions of the scale, received a strong rating. Studies associated with the ACSS-MH scale therefore provide further support for a multi-dimensional care continuity concept, since they describe 
precise statistical modelling of care continuity and receive a strong or moderate overall quality appraisal rating.

The scales 'CONNECT' and 'CONTINU-UM', on the other hand, are each associated with only one study for psychometric validation (Rose et al., 2009; Ware et al., 2003), which achieved a moderate score in the field based on the COSMIN checklist, contributing to their overall moderate rating. Taken together with the studies surrounding the ACSS-MH however, they provide further support for a multi-dimensional understanding of care continuity. The multi-dimensional framework of continuity is then the structural understanding within which I conduct the rest of this review and examine and appraise care and recovery experiences for this qualitative inquiry. I now commence review of key dimensions within this multi-dimensional construct.

\subsubsection{Experienced continuity}

Experienced continuity is defined as care perceived by the person as continuous, connected and coordinated such that no detrimental gaps in provision have occurred (Bachrach, 1981; Freeman et al., 2002). This dimension of continuity emphasises the service user's perspective on whether or not care and treatment is experienced as a continuous and seamless whole (Biringer et al., 2017). Therefore, it may be understood in terms of the overall conceptual paradigm for my inquiry based on the 'new subjective medicine' (Sullivan, 2003), and the related 'perspectivist paradigm' (Heaton et al., 2012; Sweeney et al., 2016) (see section 1.4). A number of studies $(n=5)$ denote experienced continuity as a central component within their analyses of the concept (Biringer et al., 2017; Burns et al., 2009; Freeman et al., 2002; Poremski et al., 2016; Rose et al., 2009; Sweeney et al., 2016; Ware et al., 2003).

Rose and associates (2009) developed a measure of care continuity, 'CONTINU-UM' which places particular emphasis on the service user's perspective from which basis it is entirely validated. 'CONTINU-UM' was one scale produced by the ECHO Study (Experiences of Continuity of Care and Health and Social Outcomes; Burns et al., 2007), along with 'CONTINUES' (note again that this is a name and not an acronym; Burns et al., 2013), a scale for care continuity validated from the care supporter's perspective. However, this study by 
Rose and colleagues (2009), and others related to the ECHO Study which I have reviewed, received a moderate score according to my universal quality assessment tool. Primarily this was because of a moderate rating given for the methodological qualities of psychometric validation used in producing and validating these measures. This was due to poor content validity and a lack of structural validation and hypothesis testing in psychometric testing (see Uijen, 2012). Consequently, this set of studies do not provide decisive support for the argument that experienced continuity is a central component of the overall care continuity concept.

A more robust support for experienced continuity is provided by the set of papers and studies surrounding the ACSS-MH (Adair et al., 2003; Adair et al., 2005; Durbin et al., 2004; Jensen et al., 2014; Joyce et al., 2004; 2010). Studies by Joyce and colleagues $(2004 ; 2010)$ associated with this scale received a strong rating with my quality appraisal tool, primarily due to the methodological rigour of psychometric validation contributing to a strong rating within the relevant field. They also did not receive more than one weak rating due to their having good methodological rigour and analysis, a clear statement of findings, an informative discussion and comprehensive consideration of study impact and implications.

Other studies in my review emphasising experienced care continuity, though not developing measurement scales, also received a strong overall rating according to my use of the universal quality assessment tool (Freeman et al., 2002; Sweeney et al., 2016). Similarly to the studies associated with the ACSS-MH measure, this was due to them having good methodological rigour, statement of findings and discussion.

On the basis of my appraisal of these publications, I can conclude that there is good support for the dimension of experienced care continuity as a key component of the multi-dimensional concept. Additionally, the more recent studies reviewed here have argued strongly for the importance of focusing on the views and experiences of service users as the right grounding for developing multi-dimensional concepts, models and measures of care continuity (Biringer et al., 2017; Rose et al., 2009; Sweeney et al., 2016). I will argue therefore in this thesis that experienced care continuity is a key dimension in which to appraise and understand 
participants' talk about care and recovery, echoing the perspectivist paradigm on healthcare introduced in section 1.4.

\subsubsection{Relational continuity}

The relational or therapeutic dimension of care continuity is defined as the establishment of a therapeutic relationship with one or more professionals and the service user (Belling et al., 2011; Burns et al., 2009). This concept has a high prevalence in the literature as is shown in Table 6 in Appendix 11, where it occurs in $n=13$ studies. A study by Burns and associates (2009) used a scale called the 'STAR' or 'Scale to Assess Therapeutic Relationships in Community Mental Health Care' (McGuire-Snieckus et al., 2007) to measure the contribution of the therapeutic relationship to care continuity. Factor analysis demonstrated a statistically significant role for the relational dimension within the overall concept of care continuity. Further support for this dimension is provided by other studies (Adair et al., 2003; Joyce et al., 2004; 2010; Ware et al., 2004). Relational continuity arguably plays a key role within the care continuity framework since it governs the quality of the various points of contacts between service users and professionals, and not just their quantity or frequency. The review by Haggerty and associates (2003), which provides the conceptual basis for qualitative analysis in the study by Poremski and colleagues (2016), notes that relational continuity bridges past, present and future care. It is particularly valued in primary and mental healthcare where, despite there being little expectation of establishing multiple relationships with clinicians, it is important to provide a consistent core of supportive professionals, which gives patients a sense of predictability and coherence about their treatment. The value of having a designated care coordinator who establishes a written and agreed care plan with the service user is highlighted in certain studies as a potential way in which relational continuity may be achieved, in addition to this being an important factor for cross-boundary continuity (Burns et al., 2009; Catty et al., 2011; Ware et al., 2003; see section 2.7.6).

\subsubsection{Longitudinal continuity}

The dimension of longitudinal continuity, referred to in some studies as temporal continuity (Sweeney et al., 2016), is a key dimension within the model provided by Freeman and colleagues $(2000 ; 2002)$. Longitudinal continuity is defined as having care delivered by as few 
professionals as possible, in a timely fashion and with minimal gaps in treatment (Biringer et al., 2017; Burns et al., 2009; Ware et al., 2003). A number of studies emphasise this dimension of care continuity (Adair et al., 2003; Biringer et al., 2017; Gulliford et al., 2006; Ware et al., 2004). A qualitative study by Belling and associates (2011), which focuses on continuity through the clinician's perspective, concluded that longitudinal continuity is primarily facilitated by workforce stability, whilst being hindered by poor staffing levels, high caseloads and burdensome administrative duties. Burns and colleagues (2009) operationalised longitudinal continuity as part of a factor analysis confirmation of the original multi-axial scale formulated by Freeman and associates (2000; 2002). Both these publications (Belling et al., 2011; Burns et al., 2009) were given a strong rating within my quality appraisal tool, supporting the validity of this care continuity dimension. In the case of the qualitative study by Belling and associates (2011), the only limitation and weak score in quality appraisal was due to the limited geographical location of sampling, which restricted generalisability. The strengths of this study however were a large sample size for a qualitative study $(n=113)$, a randomised selection procedure for participants in proportionate groups for each relevant profession, and a high response rate to recruitment. The study by Burns and colleagues (2009), seeking to operationalise a multi-axial model of continuity for quantitative data collection, had strengths related to the methodological rigour for psychometric validation of the scale and the quality of the introductory literature review. Due to the strengths of these two studies in particular, I found in my review strong support for the dimension of longitudinal or temporal continuity. In this thesis therefore, I will argue that longitudinal continuity plays an important role in seeking to understand recovery experiences of participants' negotiating Welsh mental healthcare systems interfaces.

\subsubsection{Cross-boundary continuity and care coordination}

Cross-boundary continuity is defined as effective coordination of care between professionals and services involving good management of links between services, professionals and components of care (Adair et al., 2005; Sweeney et al., 2016; Uijen et al., 2014; Ware et al., 2003). The literature surrounding the ECHO project identifies cross-boundary, or using their equivalent term, 'cross-sectional', continuity as one of the most important components of the care continuity construct (Belling et al., 2011; Burns et al., 2007; Burns et al., 2009; Sweeney 
et al., 2015). A major finding of a later study within the ECHO project (Sweeney et al., 2016) is that cross-sectional or cross-boundary continuity plays an integrative role within a multidimensional understanding of care continuity, contributing to good continuity in other dimensions. For example, the presence of good links between services and professionals, entailing good cross-boundary continuity, is an important factor for avoiding gaps in treatment, which could lead to longitudinal or relational discontinuities (Burns et al., 2009; Poremski et al., 2016; Ware et al., 2003). These instances of connection between separate dimensions of the multi-dimensional care continuity concept are indicative of the integrated and interconnected nature of care continuity dimensions. To an extent, certain dimensions depend upon or are causally affected by others within the care continuity framework. The factor analysis of Freeman's multi-axial model performed by Burns and associates (2009) is also indicative of the central role of cross-boundary continuity within this integrated structure.

It is important also to note that cross-boundary care continuity is closely related to the concepts of care planning (Hannigan et al., 2018) and care coordination (Burns et al., 2009; Schultz and McDonald, 2014; Sweeney et al., 2012). Indeed, good care continuity is one of the principal aims of care coordination (Burns et al., 2009; Schultz and McDonald, 2014; Sweeney et al., 2012). Care coordination, which seeks to provide better care continuity (Schultz and McDonald, 2014; Sweeney et al., 2012), may be defined as the responsibility of a professional to coordinate and plan a person's mental health care (Simpson et al., 2016a). Both care coordination and care planning may be measured within the cross-boundary continuity dimension since they both entail working collaboratively with the individual, and linking different services and professionals in order to provide a comprehensive, person-centred care plan (Belling et al., 2011; Gulliford et al., 2006; Schultz and McDonald, 2015). Care coordination is a pertinent issue for recovery-based, primary mental health care implementations such as the Mental Health (Wales) Measure 2010, since effective care coordination may not be implemented at the primary level, or it may be disrupted as patients transit from the secondary level of care (Durbin, et al., 2004; Ramanuj et al., 2015; Thielke et al., 2007).

The literature surrounding the recent COCAPP study (Simpson et al., 2015; Simpson et al., 2016a; Simpson et al, 2016b) provides robust and extensive evidence for the importance of 
care coordination and care planning within a positive care and treatment experience. However, these studies find significant levels of variation in the quality of care planning and care coordination across England and Wales (Simpson et al., 2016a; Simpson et al., 2016b). Therefore, despite strong support for the prominent role of cross-boundary continuity and care coordination within a multi-dimensional concept of care continuity, according to this review, the COCAPP studies indicate there may be a gap in understanding within this dimension of the experiences of people negotiating complex mental health services.

\subsubsection{Informational continuity}

Informational continuity may be defined as good communication between services, professionals and service users, and consistency in care plans so that providers have appropriate information about patients and resources (Joyce et al., 2004; Rose et al., 2009; Weaver et al., 2017). Informational continuity is not included in the literature surrounding the development of the ACSS-MH because here the dimension is understood to be limited to information transfer between professionals and services only, which will not be directly experienced by the service user (Durbin et al., 2004). It may however be experienced indirectly within other dimensions of care continuity relevant to the service user. For example, poor longitudinal care continuity experienced by the service user may be indicative of underlying issues concerning informational discontinuity. Additionally, the negative experience of having to repeat treatment history at each new point of care should provide sufficient grounds to infer the presence of poor informational continuity between service providers. For these reasons, the dimension of informational continuity is considered to be relevant to the understanding of care continuity produced by this review and undertaken in this inquiry.

\subsection{Conclusion}

In this chapter, I have described the background for my study inquiry based on review and appraisal of literature in the three key subject areas of complex mental health service systems and related policy, recovery as the key focus of policy, and care continuity as the key element in recovery-based services. I have also described both inductive and deductive strategies employed in my scoping review approach, my method of thematic evidence synthesis 
informing a narrative review, and the use of my quality assessment tool in appraising the evidence.

In the first part of the review, I identified literature describing how mental health services, including in Wales, may be understood in terms of complex systems. The impact of policy, for instance, the Mental Health (Wales) Measure 2010, was considered to be an exacerbating effect on existing service complexity. The overall picture of services was therefore generated of an increasingly complex and fragmented mental health system, partly contributed to by a succession of transformative policy programmes.

In section 2.6, I reviewed literature concerning recovery as the key focus of policy. The history and contested nature of the concept was described in relevant literature. This then led me to consider policy which seeks to implement recovery-based services. I considered the argument within some literature that such recovery-based policy programmes are influenced by a neoliberalist agenda.

In section 2.7 , I focused my review on care continuity in view of it being a conceptual perspective through which healthcare and recovery experiences may be examined. I concluded that this conceptual perspective should be multi-dimensional and detailed some of the key components of this framework.

In covering these three areas, and providing a logical link between them, this review provides a background of understanding based on existing literature relevant to this study. This inquiry may therefore be conceived with the intention to develop new knowledge about care continuity experiences of recovery-oriented healthcare in the context of complex mental health systems, which are impacted by transformative, recovery-focused policy implementations. In the next chapter, I build on the background for the inquiry provided in this and the previous chapter, by describing the methodology and methods I used to achieve the aim and objectives of the study. 


\section{Chapter 3: Methodology and methods}

\subsection{Introduction}

In this chapter I describe the methodology and methods used to achieve the aim and objectives of the study. I make a distinction between methodology and method in this chapter based on the understanding that method refers to the practical considerations of the study, whilst methodology concerns the theoretical and philosophical underpinnings underlying the research inquiry (Miller and Brewer, 2003). I therefore explain the research design in terms of the chosen methodological position which forms the basis for the specific methods I employ.

The structure of this chapter is as follows. Firstly, I reiterate the study aims and objectives and then go on to describe the two-phase study design. This is followed by a discussion of the precise nature of the research inquiry into healthcare in Welsh mental health services. I then discuss background and rationale for the study inquiry. An explanation for the philosophical and paradigmatic underpinnings of a qualitative, constructionist methodology is then provided. This forms the basis for my chosen method of qualitative, thematic analysis of in-depth semistructured interviews (Braun and Clarke, 2006; Bryman, 2016). I describe details of this method in terms of data collection (interviews, recruitment and sampling strategy), and the process of data analysis (coding and development of themes). Ethical issues related to the study are also discussed. Following this, I describe my method of thematic discourse analysis (Braun and Clarke, 2006; Braun et al., 2014; Singer and Hunter, 1999). I also discuss evaluative criteria concerning quality, rigour and credibility of qualitative findings. This leads me to specify theoretical generalisation (Mitchell, 1983; Seale, 1999) as the basis for new knowledge to be generated by this piece of research.

\subsection{Study design}

The research design is properly understood as having its roots in an appropriate philosophical foundation (Creswell and Plano Clarke, 2011). In this chapter, I set out philosophical assumptions based on the underlying paradigm of perspectivism introduced in Chapter one to shape the process of research and the conduct of the research inquiry (Creswell and Plano 
Clarke, 2011). This theoretical underpinning, in combination with the literature review, forms the conceptual background to the research project.

The study involved two phases:

- Phase 1 involved semi-structured interviews of service users with a of history serious mental illness (SMI) transiting between secondary and primary mental health care levels in West Wales. These individuals were recruited through a variety of third sector organisations and day centres at various locations in West Wales.

- Phase 2 involved semi-structured interviews of managers, workers, practitioners and volunteers in third sector organisations with experience of care and support of service users at the interface of primary and secondary mental health care levels in West Wales.

Conducting the research in two phases enabled me to develop my work in the context of a multiple perspectives paradigm (Heaton et al., 2012; Rose et al., 2006; Sweeney et al., 2016). This accords with the perspectivist paradigm (Heaton et al., 2012; Sweeney et al., 2016) and new subjective medicine (Sullivan, 2003) described in section 1.4, which forms the conceptual underpinning of this project. A multiple-perspectives paradigm (Rose et al., 2006; Sweeney et al., 2016) is an emerging paradigm in healthcare which seeks to co-construct continuity of care through the interaction between service users' and professionals' perspectives (Heaton et al., 2012). The two-phase nature of the research design seeks to achieve this multiple-perspectives interaction.

\subsubsection{Research question, aims and objectives}

In Chapter one, I set out the aim of this study and I summarise it here with regard to the overarching research question:

"What are the experiences of care continuity and recovery for people at the interface of primary and secondary mental health care in Wales?" 
The overall aim of this study therefore is to provide new evidence on the organisation and experience of care continuity and recovery at the interface between primary and secondary care in Wales.

The study aims and objectives are:

- To produce an evidence synthesis of care continuity and recovery literature relevant to persons with a history of serious mental illness, related to movement across the primary and secondary mental health interface. This was provided in the literature review in the previous chapter, in addition to the study context and background provided in section 1.2

- To investigate how individuals experience the organisation and delivery of care continuity and recovery while moving across the interface between primary and secondary sectors as a result of current service transformations and trends in Wales, considering the policy-context of Mental Health (Wales) Measure 2010.

- To investigate these experiences of health and care using a qualitative research approach within a perspectivist epistemological paradigm.

A fundamental principle of study design is that there should be a credible link between the methodology and the nature of the research inquiry being undertaken (Bryman, 2016; Silverman, 2014). In the next section therefore, I will describe philosophical underpinnings of the methodology, which I deem to be appropriate for this inquiry into care continuity and recovery experiences for people transiting between primary and secondary mental healthcare levels in Wales.

\subsection{Methodology and philosophical underpinnings}

In this section I use a framework for methodological study design provided by Creswell and Plano Clark (2011), based on Crotty (1998), to describe the philosophical underpinning of constructionism for this research. Building on the perspectivist philosophical foundation I laid out in Chapter one, I argue for an interpretivist approach to underpin this qualitative inquiry, 
as opposed to a naïve realist approach based on positivism (Ritchie et al., 2013). Primarily, I advocate constructionism as an appropriate methodological approach to conduct an interpretivist inquiry into people's care continuity and recovery experiences. Following on from this, I discuss criticisms of constructionism and how these issues may be resolved by reconciling critical realism with constructionism.

A useful framework for understanding how philosophical assumptions may underpin the design of a research inquiry is provided by Crotty (1998). Crotty proposes that there are four main levels for conceptualising the philosophical underpinnings of a research study: the epistemological or philosophical level, the theoretical level, the methodological level and the level at which specific methods are employed (Crotty, 1998). Creswell and Plano Clark (2011) adapt Crotty's framework into a tiered model of four levels, using Thomas Kuhn's (2012) concept of a 'paradigm' to describe the fundamental philosophical level or viewpoint which underpins the theoretical and methodological levels of the research inquiry (Creswell and Plano Clark, 2011; Guba and Lincoln, 1998). Creswell and Plano Clark's (2011) model is provided below in Figure 3.

\section{Figure 3: Four levels of research study development}

\section{Paradigm worldview}

(beliefs, e.g., epistemology, ontology)

\section{Theoretical lens}

(e.g., feminist, racial, social science theories)

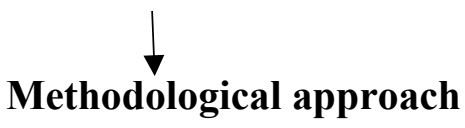

(e.g., ethnography, experiment, mixed methods)

\section{Methods of data collection}

(e.g., interviews, checklists, instruments)

(Creswell and Plano Clark, 2011, p.39; adapted from Crotty, 1998) 
It can be seen from this tiered model that the four successive levels are founded upon each other. The foundational, paradigmatic level forms the basis for the theoretical level, which in turn forms the basis for methodological approach. Finally, the level of the practical aspects of method which are employed in conducting the research project is based upon the three previous levels. This chapter is structured according to Creswell and Plano Clark's (2011) tiered model. Firstly, I discuss the paradigmatic foundation and the theoretical perspective within which this piece of research is conceived. Building on this, I discuss methodology in terms of the various options available for the researcher to undertake an inquiry. These options include broad approaches such as qualitative, quantitative or mixed methods research, and more specific approaches within these broad methodological traditions, such as grounded theory, discourse theory, ethnography or thematic analysis (Braun and Clarke, 2006; Bryman, 2016). I discuss my rationale for decisions I make about which of these options and approaches I adopt. Then, after discussing ethical issues (section 3.4), I discuss my method referring to the specific methods of data collection and analysis I employed as a researcher based on the established methodology (sections 3.5 and 3.6). Also relevant to my method are issues of credibility and validity, which are discussed in section 3.7. In the following series of sub-sections, I discuss the first two levels of Creswell and Plano Clark's (2011) framework for methodological study design, concerning paradigmatic foundation and theoretical perspective.

\subsubsection{Interpretivism in sociology}

Interpretivist approaches within sociology were developed in the nineteenth and twentieth centuries in opposition to the once-dominant philosophy of positivism (Schwandt, 2000). Positivism has traditionally been associated with natural science inquiry into the physical world (Bishop, 2007; Harrington, 2005), and has had a major influence on the early development of social science inquiry (Ritchie et al., 2013). Indeed, Auguste Comte, who was one of the founders of social science, and originator of the term 'sociology', popularised the term 'positivism' or 'positive science' to describe his view that social phenomena could be studied on the same empirical basis as the objects of natural science (Bishop, 2007; Crotty, 1998; Delanty, 2005; Harrington, 2005). One of the early critics of positivism in social science was the late nineteenth century thinker, Wilhelm Dilthey (1976). Dilthey's thought was a reaction against positivism in sociology, promoting the view that this scientific approach was 
inappropriate for the study of human beings who are not inanimate objects but have the capacity for meaning and intentionality (Dilthey, 1976). Dilthey was influenced by the school of neoKantians and their critical philosophical position that 'things-in-themselves' are unknowable since epistemology should be confined to phenomena or 'things-as-experienced', referring to a dynamic realm of interaction between subject and object (Manicas, 2006; Protevi, 2005). This notion of epistemology as constituted by the interactive plane between subject and object was later taken up by phenomenological philosophers such as Heidegger and Merleau-Ponty (Heidegger, 1967; Merleau-Ponty, 2013 [1962]; Mulhall, 1996). This led to their radical rejection of the Cartesian dichotomy between subject and object.

Wilhelm Dilthey's critique transposed the critical philosophical interpretivism of the neoKantians to the realm of social theory and was an early version of what has been called the 'interpretive turn' in sociology, which countered the then-dominant positivist position in social science (Delanty, 2005). Interpretive or hermeneutical sociology was developed further by a various twentieth century social theorists, including Max Weber, Peter Winch and Anthony Giddens (Bishop, 2007). The interpretivist position in social theory asserts that positivistic, natural scientific explanations fail to capture the meanings, intentions and interactions which accompany social phenomena (Harrington, 2005). According to this view, a positivist approach to developing knowledge may inadequately describe and even distort the nature of human behaviour, since it strips away important contextual and intentional information (Taylor, 1993).

Max Weber also emphasised the interpretative dimension within social theory. He introduced the concept of 'Verstehen' to describe the interpretative, intentional and empathetic context of human action (Manicas, 2006). He contrasted this with 'Erklaren' which refers to causative explanation in accordance with the positivist understanding. In modern sociology, writers such as Peter Winch (1990) have added to interpretivist sociology by arguing that a proper understanding of human action requires an account of their motives and intentions, manifested in language and behaviour, which goes beyond a mere causal explanation of human beings (Bishop, 2007). According to Winch (1990), human activity consists in cooperative activities guided by intersubjective meanings and values rather than by causal laws (Bishop, 2007). 
Anthony Giddens also drew on the interpretivist view within sociology, introducing the concept of the 'double hermeneutic' to refer to the interpretive capacity of both the research inquirer and research subject (Giddens, 1984). Giddens' concept of the double hermeneutic points towards a clear distinction between natural science and social science inquiry. Unlike in natural science, "social scientists are actors engaged in an intelligent, social activity of studying intelligent, social activities of fellow human beings. Put another way, the interpretive nature of social inquiry is a two-way street" (Bishop, 2007, p.53). One of the consequences of this is that social scientists will not only bring their cultural biases, values and interpretations to scientific inquiry but they will also be influenced by the cultural biases, values and interpretations of the subjects they are studying.

John Stuart Mill has argued that the only difference between natural science and social science is that of complexity, and that the social sciences may therefore be understood in a similar way to the natural sciences (Bishop, 2007). However, considering the 'interpretative turn' in sociology, and the thinking of the many social scientists and philosophers which has contributed to this, it seems far more insightful to consider that natural science and social science are qualitatively different from one another. This qualitative distinction appears to centre on the interpretive capacity of human beings, a feature not possessed by inanimate objects of natural science. A methodological approach which is suited to natural science, such as positivism, and which does not properly account for this interpretive dimension, at best limits the scope of scientific inquiry and at worst distorts the important issues of social and political life (Bernstein, 1976). On the basis of this consideration of the 'interpretative turn' in sociology, I concluded that an interpretivist (or hermeneutic) understanding of human beings would be the appropriate paradigmatic worldview or philosophical underpinning within which to conceive and comprehend the methodological basis for this research study. This builds on the underlying perspectival paradigm which I introduced in section 1.4.

\subsubsection{Social constructionism}

Social constructionism is identified by Schwandt (2000) as a major interpretivist approach which may be adopted as a methodological paradigm for sociological research inquiry. It is opposed to positivism since it rejects an ontological commitment to a singular, objective reality 
in favour of embracing multiple, localised realities (Creswell and Plano Clark, 2011; Guba and Lincoln, 1994). Indeed, the rise of constructionism as a methodological approach can be seen as a response to several criticisms of positivism (see Guba and Lincoln, 1994), and its subsequent fall from dominance within the natural and social sciences (Houston, 2001; Kuhn, 2012; Rorty, 1980). The central position of social constructionism is that the social world is manufactured through human interpretation, interaction and language (Berger and Luckman, 1991; Miller and Brewer, 2003). Society therefore is not a domain which pre-exists human interpretation and the generation of meaning but is rather produced by human interpretation, interaction and language (Berger and Luckman, 1991; Houston, 2001). Vivien Burr (2003) summarises this position of social constructionism:

"It is through the daily interactions between people in the course of social life that versions of knowledge become fabricated. Therefore, social interaction of all kinds, and particularly language, is of great interest to social constructionists. The goings-on between people in the course of their everyday lives are seen as the practices during which our shared versions of knowledge are constructed."

(Burr, 2003, p.4, my emphasis added)

I have emphasised Burr's (2003) claim that language is the key medium for social interaction and interpretative action. According to constructionism, language is a precondition for thought and reality, rather than a consequence of it, since the concepts and categories of language are necessary pre-requisites for people to make sense of the world (Burr, 2003). It is language that makes thoughts and concepts possible and not the other way around, since language provides a means of structuring the way in which the world is experienced and understood (Andrews, 2012). Martin Heidegger, who pioneered modern interpretivism in the field of philosophy, famously wrote: "Words, like the chisel of the carver, can create what never existed before rather than simply describe what already exists. As a man speaks, not only is the thing which he is declaring coming into existence, but also the man himself' (quoted in Walker, 2006, p.34). In other words, language has a fundamental role in constructing both the world and the individual who apprehends it. We are world- and self-interpreting beings whose being and context is constituted by language (Schwandt, 2000). This theoretical position is the basis for discursive psychology, an approach within discourse analysis which focuses on how people use language to create and negotiate representations of the world and simultaneously construct 
their own identities in talk (Antaki et al., 2003; Jørgensen and Phillips, 2002; Potter and Wetherell, 1987).

Ian Hacking writes about teenage pregnancy as one example of how social phenomena are constructed through interpretative activity and language (Hacking, 2003). Although women have been conceiving under the age of 18 for thousands of years, teenage pregnancy as a twentieth century social phenomenon is something which has come into being as a result of human interaction and meaning-making behaviour. In a similar manner, many of the key concepts in my research inquiry, such as 'recovery' and 'care continuity' may be identified as social constructs.

Constructionist (and interpretivist) philosophies have had a major influence on qualitative research methodologies such as grounded theory, discourse analysis and narrative research (Braun and Clarke, 2006; Corbin and Strauss, 2008; Riessman, 2008). Clearly, the constructionist view that language is a precondition for thought and apprehension of reality has major implications for the nature of qualitative analysis of language within interview transcripts. Discourse analysis, in particular, is an approach to qualitative analysis which focuses on the constructionist role of language in human life and interaction. Consequently, it is one of the most widely used qualitative approaches employed in research conceived under a constructionist paradigm (Jørgensen and Phillips, 2002).

It should be noted that the broader methodological category of qualitative research is generally associated with interpretivist and constructionist paradigms (Guba and Lincoln, 1994; Schwandt, 2006). This is in contrast with the general association which can be made between quantitative research and a positivist outlook. Bryman's (2016) summary table of the epistemological and ontological characteristics of quantitative and qualitative research strategies illustrates these contrasting characteristics of qualitative and quantitative research: 
Table 2: Fundamental differences between quantitative and qualitative research strategies

\begin{tabular}{|l|l|l|}
\hline & Quantitative & Qualitative \\
\hline $\begin{array}{l}\text { Principal orientation to the } \\
\text { role of theory in relation to } \\
\text { research }\end{array}$ & Deductive; testing of theory & $\begin{array}{l}\text { Inductive, generation of } \\
\text { theory }\end{array}$ \\
\hline Epistemological orientation & $\begin{array}{l}\text { Natural science model, in } \\
\text { particular positivism }\end{array}$ & Interpretivism \\
\hline Ontological orientation & Objectivism & Constructionism \\
\hline
\end{tabular}

(Bryman, 2016, p.32)

Table 2 indicates that quantitative research has an epistemological orientation towards positivism and an ontological orientation towards objectivism. Qualitative research on the other hand has an epistemological orientation towards interpretivism and an ontological orientation towards constructionism. Quantitative work tends to be more deductive, where a hypothesis is subjected to rational, empirical testing, whilst qualitative research builds up inductively from the data so as to provide more general inferences and produce new theory. It should be recognised however that the association made by Bryman (2016) between quantitative or qualitative methodologies and positivist or interpretivist epistemological paradigms respectively should best be understood as an orientation and not as a necessary relationship. Indeed, quantitative studies may involve a degree of interpretation and qualitative studies may incorporate positivism and quantification (Crotty, 1998; Seale, 2011; Tesch, 1990). However, the tendency for qualitative research to be more interpretivist and quantitative research to be more positivist is an important guideline for research design being clarified in this chapter.

On the basis of the theory described above, and reflexive considerations of my own interpretative contributions as a researcher to this study (see section 1.4), I came to the conclusion that, at both the paradigmatic and methodological levels of Creswell and Plano Clark's (2011) framework, it was most appropriate for me to embrace an interpretive, constructionist philosophical underpinning for a qualitative methodology as the basis for my inquiry into participant experiences of mental healthcare in Wales. A qualitative approach is suited to exploratory research into an area which has not been studied to a great degree, upon 
which basis exploratory theory and inductive inferences may be developed (Bryman, 2016; Creswell, 2013; Ritchie et al., 2013). Since this is the first academic study of care continuity and recovery experiences for people at the interface of primary and secondary mental health care in Wales, I concluded that a qualitative, exploratory approach was most appropriate for my research. Additionally, a qualitative approach is the method of choice for what I was seeking to carry out, namely, accessing people's experiences via their accounts of encounters in systems of care. Qualitative inquiry within a constructionist paradigm is a widely used approach for this purpose since it captures the hermeneutic meaning and context of social phenomena experienced by individuals, and may facilitate an in-depth understanding of their subjective viewpoints (Denzin and Lincoln, 1994). Because social constructionism emphasises the role of language and other interpretative activities as a precondition for structuring our social worlds, it is ideally suited to identify deep, underlying structures and practices which determine the complexities of everyday life, as well as how people understand and experience their social worlds. Consequently, I concluded I would be able develop a more satisfactory and informative sociological account of mental healthcare phenomena on the basis of a qualitative, constructionist methodology with an interpretivist paradigm and philosophical underpinning. I therefore chose this approach to be the basis of my methodology.

\subsubsection{Criticisms of constructionism}

I now focus on some of the criticisms of constructionism as a methodological and philosophical position. The consideration of criticisms I present here leads me to broaden my constructionist approach by incorporating into it aspects of critical realism. The emphasis on perspectivism and interpretivism within constructionism can lead to the countenance of unlimited multiple realities and an associated relativism (Bishop, 2007; Guba and Lincoln, 1994). This is particularly the case with radical and postmodernist versions of constructionism (Delanty and Strydom, 2003; Luhmann, 1995) where multiple constructed accounts can each claim equal legitimacy and there is no reason to prefer one version over another (Andrews, 2012; Guba and Lincoln 1994). Richard Rorty is one expositor of the relativism of the radical constructionist position and asserts that there is "no criterion that we have not created in the course of creating a practice, no standard of rationality that is not an appeal to such a criterion, no rigorous argumentation that is not obedience to our own conventions" (Rorty, 1982, p.xlii). 
In the ethical sphere, such radical interpretivism and relativism may be viewed in a positive light, since it makes provision for a hermeneutic understanding of human beings, and does not strip away contextual and interpretative information, which I have argued is vital aspect of understanding social individuals and human nature (Taylor, 1993). Radical constructionists such as Norman Denzin and Kenneth Gergen have extended this ethical stance into a politicised agenda of democratisation, emancipation and social progress (Denzin, 2016; Denzin and Giardina, 2008; Gergen, 1994). This is coupled with a critique of positivism as reductionist and even tyrannical since the constraints of this paradigm affords little or no freedom for alternative views to those established as objectively and unassailably true (Denzin, 2016; Denzin and Giardina, 2008).

However, the counter-argument to all this is that an abandonment of objectivity which constructionism might entail may lead to an absence of substantive or normative foundations, and consequently a nihilistic philosophy in which all epistemological or ethical discussion is rendered pointless (Bishop, 2007). Schwandt (2000) argues as follows:

"Absent any criteria for deciding across various frameworks which is the better (the more just, more democratic, and so on) practice and if there is no epistemic gain or loss resulting from this comparison, why would we bother to engage in the conversation?"

(Schwandt, 2000, p.200)

Constructionists counter that their position is not irrational or relativistic because there is an inherent stability of values and viewpoints created by the requirements of intelligibility shared in a particular community (Rorty, 1985). However, a notion of truth based purely upon intersubjectivity is still vulnerable to a gradual erosion of values and introduction of instability over a period of time. Without any sort of objective anchoring, a radical constructionist position is vulnerable to drift across the entire ethical spectrum, and also, ultimately to a complete epistemological fluidity, two scenarios which I decided were not acceptable as implications of my work. Indeed, as I carried out this healthcare research project, my moral and epistemological stance developed into one increasingly grounded in the authenticity of the lived experience of recovering service users (Deegan, 1998; Middleton, 2007; Repper and Perkins, 2003). 


\subsubsection{Constructionism and critical realism - a reconciliation and solution to the problem of objectivity}

Despite these issues, closer examination of the constructionist paradigm reveals that it is not necessarily opposed to incorporating any element of objectivity whatsoever. Constructionism should be distinguished from the philosophical view of idealism, whose epistemological position is one of solipsism, according to which reality is in its entirety a creation of the mind (Delanty, 2005; Delanty and Strydom, 2003; Guba and Lincoln 1994; Kenny, 2006). Crotty (1998) criticises a great deal of qualitative research as being aligned to this idealist distortion of constructionism rather than to a more accurate understanding in which there does exist a reality beyond human perception. In this vein, Crotty (1998) describes much qualitative research as being guilty of a "rampant subjectivism" in moving from "a study of phenomena as the immediate objects of experience into a study of experiencing individuals" (p.48). This is a subtle distinction between different approaches to conducting qualitative research about people's experiences, but in my view, a crucial one. According to Crotty (1998), constructionism properly conceived should be "a study of phenomena as the immediate objects of experience" (p.48, my emphasis added). In other words, Crotty asserts there should be a sense of phenomena pointing towards an objective reality, within a qualitative and constructionist epistemological conception. This is a description of intentionality, which is an important notion within existentialist phenomenology (Cooper, 1999), and a philosophical position which Crotty uses to describe constructionism as properly conceived in his view. According to the principle of intentionality, all mental states are consciousness 'of' or 'about' something (Lyotard, 1991; Scruton, 1994). Intentionality therefore indicates a fundamental and necessary link between subjectivity and objectivity. A sharp distinction between subjectivity and objectivity is in fact a false dichotomy, according to this view. As Crotty (1998) puts it, "intentionality is a radical interdependence of subject and the world" (p.45). Therefore, the meaning and concepts with which we apprehend the world are not created within the boundaries of subjectivity, in the solipsistic sense of idealism (Delanty, 2005; Kenny, 2006; Rorty, 1980). Rather, human beings construct meaning out of the world and its objects to which subjectivity is bound by the principle of intentionality. Constructionism does not deny the existence of an external reality but holds that the empirical world is known (only) through the interpretative role of constructions. The world and its objects are real, but they are only known in a meaningful way via the categories with which we understand and construct the world 
(Jørgensen and Phillips, 2002). Therefore, it is not reality itself which is socially constructed but the categories or theories about reality which are constructed (Brown, 1995; Rogers and Pilgrim, 2014).

For the purposes of my own research project, I felt more comfortable with this existential or intentional version of constructionism, clarified by Crotty (1998). It seemed to me to be a more philosophically accurate version of constructionism insulated from some of the difficulties of relativism and solipsism associated with an idealist conception of constructionism. This also fitted in with the perspectivist paradigm I laid out in section 1.4. To clarify this philosophical position further for my research, I investigated contextualist conceptions of constructionism in my methodological preparation. Contextualist constructionism acknowledges the ways in which the broader social context and the limitations provided by a notion of objectivity and reality may impinge upon constructed meanings (Braun and Clarke, 2006). According to Willig (1999), who provides a contextualist position, relativism may be avoided in social constructionist research by incorporating the epistemological viewpoint of critical realism (Bhaskar, 1978).

In essence, critical realism can be understood as a critique of naïve realism's conflation of the epistemological and ontological levels of appearance and reality; this conflation is referred to as the 'epistemic fallacy' (Danermark et al., 2001; Rogers and Pilgrim, 2014). In naïve realism, reality is 'flat' and one-dimensional, whereas in critical realism it has a 'deep' structure of three levels consisting of experienced events; the actual level, comprising all events whether experienced or not; and at the deepest level, intransitive structures such as biochemical, economic or social structures (Danermark et al., 2001; Houston, 2001; Ritchie et al., 2013; Willig, 1999). Willig's (1999) contextualist constructionism incorporates critical realism which provides an objective context delimiting the range of possible constructions. According to this view, constructions are "historically and culturally variable ways of making different kinds of sense of the phenomena and events generated by intransitive structures" (Willig, 1999, p.45). Intransitive structures are the deepest layer of critical realist ontology corresponding to underlying objective features of the world. The objective reality of intransitive structures is understood within critical realism to be transcendent and therefore above or beyond the range of normal or physical human experience. Consequently, intransitive structures cannot be 
known directly, but can only be imperfectly and probabilistically known by human beings as constructions. Intransitive structures do not directly determine social phenomena or our constructed understanding of them. Rather they generate tendencies or potentialities which may be realised to a certain extent by a particular construction (Houston, 2001; Willig 1999). Willig's (1999) contextualist constructionism allows us then to posit that these probabilistic ways of knowing are constructed theories about reality.

Combining constructionism with critical realism in this way provides a notion of objectivity which provides some safeguarding against rampant subjectivism and unbridled relativism. Crucially, this combination means that different constructed accounts may be more or less valid, since critical realism embraces the idea that there may be different degrees of validity about knowledge and perception (Danermark et al., 2001; Houston 2001). This had an important effect upon the data analysis stage of my research since I felt able to preserve a notion that a particular construction or interpretation had a greater or lesser correspondence to reality or was more or less rational. In this way, some ideas and concepts discussed in the course of interviews were considered by me to be more valid because they exhibited a greater degree of correspondence to reality, or were more or less reasonable. This reality and rationality could not be perfectly known, according to the tenets of critical realism, but could be imperfectly apprehended by reference to evidence presented in supporting literature and associated theory, as well as by reference to the accounts of other participants and my own clinical experience. It was in this manner that I conducted thematic discourse analysis of qualitative interviews framed within this epistemological understanding of a contextualist, critical realist version of constructionism.

Summing up, it is the position of this thesis therefore, along with writers such as Delanty (2005), Rogers and Pilgrim (2014) and Beck (1996), that the divide between constructionism and critical realism is in fact a false dichotomy and that these paradigmatic positions may be combined to create a hybrid paradigm (Denzin and Lincoln, 2017). Both these positions can be understood to view reality as having an independent though transcendent existence, with knowledge about it being generated through interpretation. By combining these two paradigms, it is intended that a middle-ground may be reached between the objectivity of naïve realism and the perspectivism of radical constructionism. In achieving this, the potential relativism of 
radical constructionism may be avoided whilst at the same time conserving the insights which are offered by understanding knowledge to be generated through interpretation.

\subsubsection{Reflexivity and social constructionism}

Reflexivity in research arises naturally out of such a constructionist paradigm since this emphasises the researcher's perspective on phenomena and therefore the researcher's positioned view of the world. This methodological reflexivity ties in with the more personal account of reflexivity concerning how I am located within the study in section 1.4. Here, I described how the researcher's perspective and positionality can only be properly understood by reflecting on the researcher's cultural biases, values and interpretations which emerge from his social, political and historical context (Bryman, 2016; Guba and Lincoln, 1994). Additionally, the researcher is seen as an active agent producing meaning and knowledge about the world in the process of co-constructing it (Denzin and Lincoln, 1994). Reflexivity, understood in this way, may be closely associated with the methodological reflexivity of a research inquiry based on social constructionism. In support of this point, Delanty and Strydom (2003) write:

"It is increasingly being more and more recognised that our knowledge of social reality is a construction of social science in the sense that social scientific knowledge is a reflexive knowledge which constitutes its object."

(Delanty and Strydom, 2003, p.139, my emphasis added)

Consequently, constructed knowledge and meaning will be properly understood by reflecting on what the researcher and participants bring to the meaning-making process of encountering reality. The practical implications of this are that reflection should be carried out throughout the course of this research project, conceived within the paradigm of constructionism, which is what I endeavoured to do (Coffey and Atkinson, 1996). Additionally, my developing a sense of theoretical sensitivity was conducted in the spirit of reflection since my cultural biases, values and interpretations affected my interpretation and application of theories I deemed to be relevant to analysis. In this way, my engagement with theories providing a context for analysis was to an extent a personal and therefore a reflexive undertaking (Rivas, 2011). 


\subsubsection{Theoretical perspective}

I have established in this sub-section my methodological stance and philosophical underpinning for this research project on the basis of the arguments provided above. My epistemological, philosophical and paradigmatic underpinning is that of interpretivism which I argue is most appropriate for this piece of sociological healthcare research. I have also articulated my choice of constructionism as the particular version of interpretivism I adopt, to which is added a context of critical realism (Willig, 1999). These positions correspond to the foundational level of Creswell and Plano Clarke's (2011) four-level tiered model. I have also described my reasons for conceiving this study as a qualitative, exploratory research inquiry, corresponding to the methodological level of Creswell and Plano Clarke's (2011) model. I develop the details of this methodology further in subsequent sections of this chapter where I will describe my intention to perform thematic analysis (Braun and Clarke, 2011) incorporating discourse analysis (Edwards and Potter, 1992; Fairclough, 2003; Gee, 2010). At this stage however, I want to briefly focus on the second level of Creswell and Plano Clarke's (2011) model which refers to the theoretical lens or perspective through which research phenomena are understood. Within a qualitative, constructionist inquiry, examination of relevant subjectareas and theory is important for the researcher to develop theoretical sensitivity to provide a context for data analysis (Mitchell, 1983; Rivas, 2011; Seale, 2011; Silverman, 2011). The literature review undertaken in the previous chapter was carried out with this purpose in mind, providing a basis for relevant subject areas and theoretical perspectives to provide a context for both data collection and analysis. The previous chapter can therefore be seen as fulfilling the requirements of the second level of Creswell and Plano Clarke's (2011) model. In performing my literature review, I also developed an argument connecting subject areas which would form an underlying rationale for the focus of qualitative analysis. This argument can be summarised as stating that mental health services impacted by policy may be understood through complex systems theory, that recovery is the key focus of policy, and that care continuity is the key element in recovery-based services. The argument developed in the literature review therefore can be considered to form the overarching theoretical lens or perspective with which I understand and analyse data. In addition, whilst the foundation for the theoretical lens is established in the literature review, appropriate theory is developed further from this basis in combination with data analysis provided in subsequent findings chapters. 
In the next section, I describe the ethical issues I considered when designing and conducting this research study. In sections 3.5 and 3.6 following this, I will describe my method of data collection and analysis, which is based upon the philosophical and methodological underpinning I have described so far in this chapter.

\subsection{Ethical issues}

This research project was conducted according to ethical standards for all medical and healthrelated research, set out by the Declaration of Helsinki (WHO, 2001a). An application for ethical permission to conduct research was made to the Swansea University College of Human and Health Sciences Research Ethics Committee. This application was successful and permissions were granted by this committee. Special ethical considerations were made concerning the participation of vulnerable adults in this research study, where there may be issues with impairment of capacity. I focused on ensuring that participants had the capacity to provide informed consent for the research I was conducting, in direct consultation with staff who were attending the various third sector settings where I recruited participants. All participants were given detailed written information on the study and asked to sign a consent form indicating their willingness to participate.

Evidence gathered was protected by anonymisation and other strategies which obstruct the identification of participants, in order to ensure confidentiality. Information in either a physical or electronic format was stored in a secure manner. It was made clear to potential participants that participation was voluntary and that withdrawal was possible at any time before data was anonymised. Participants were informed however that there are limits to confidentiality, where for example there is revelation of harm to self or others, or illegal activity.

The researcher underwent a DBS check with Swansea University at commencement of PhD, which is held by the Human Resources Department. This was necessary to ensure protection of vulnerable adults. 


\subsubsection{Potential risks to participants}

This research study was conducted according to ethical standards for all medical and healthrelated research as outlined in the Economic and Social Research Council (ESRC) Framework for Research Ethics (ESRC, 2015). The BSA Statement of Ethical Practice states that researchers must "consider carefully the possibility that the research experience may be a disturbing one" for participants, and that the researcher should attempt to minimise or alleviate any potential distress to participants (BSA, 2002, p.4). Special precautions must also be taken for participants who are vulnerable persons (Beauchamp and Childress, 2013). Achieving research objectives was therefore considered to be of a lower priority than concerns about the wellbeing and safety of participants.

I considered the possibility that sensitive issues or experiences may be raised by participants during the course of semi-structured interviews which may lead to some emotional distress (Richards and Schwartz, 2002). A couple of times this did occur and it aided me greatly in handling these situations that I have clinical experience of dealing with individuals with SMI, and am a fully qualified mental health nurse registered with the Nursing and Midwifery Council (NMC). Thus, I have developed professional skills through training and clinical experience which were employed in order to take an empathetic approach, de-escalate any potentially upsetting situations, and guide the interview carefully so that it did not become therapeutically disruptive for the participant. In one case, the interview had to be ended because the participant recalled traumatic experiences which had not been properly resolved through therapy and support. This interview was brought to a close in a timely manner and I provided appropriate support in liaison with third sector staff. I also took care to ensure that a power imbalance in the research relationship due my professional status as a mental health nurse did not give rise to a sense of obligation, pressurisation, coercion or any form of manipulation (Hewitt, 2007).

\subsubsection{Ensuring confidentiality and anonymity}

Information gathered through qualitative research was protected by anonymisation which protected the identification of participants, in order to protect their privacy (WHO, 2001a). The identity of research participants was not recognisable in research reports, presentations or publications. I also ensured that workers for the transcription service I hired, 
www.UKTranscription.co.uk, signed a confidentiality form, although I also used code numbers and not names to introduce participants in audio recordings (see section 3.5.3.2 regarding transcription).

Information collected was kept confidential in a variety of ways. I was the only person who could access personal information that could identify participants. Number codes were recorded at the beginning of interviews and used as identifiers on transcripts. In this way, interview audio recordings and interview transcripts did not include participants' names. These number codes were placed by the names of participants on completed consent forms (a copy of the consent form is provided in Appendix 4). Completed consent forms, the list of names and contact details for participants and the appointment checklist were kept in the same secure location on University premises. Digital audio recordings of interviews were also kept in this secure location. Electronic copies of transcripts were kept in this secure location as well. Data collected for this research study will be retained securely for five years from the end of this study and then destroyed. Digital audio files of the interview recordings and interview transcripts (word documents) were stored in encrypted format on the university's secure server which is password protected.

\subsubsection{Informed consent}

Potential participants were deemed to have capacity to understand the nature of the research discussed in the participant information sheet, as well as the consequences and risks of participating (ESRC, 2015). This was a prerequisite for participants to give informed consent. A person has capacity if they have a general understanding of decisions they need to make and why they need to make them, which in this case was the decision to participate in research interviews. The participant must be able to retain and weigh up the information relevant to the decision and must be able to communicate their decision adequately (Mental Capacity Act, 2005). I was aware of the possibility that the capacity of a person with a mental health problem may fluctuate if the person's mental health declines. I used my professional skills and clinical experience to assess capacity at the time of the interview, and to be continue to be sensitive to changes in participants' status of providing voluntary informed consent. However, at no times 
were there any issues concerning mental capacity, which underlines the point that a person with a mental illness does not necessarily lack capacity (Scottish Government, 2001).

Those expressing an interest in taking part in the research project were asked to confirm their participation by signing and dating the consent form to demonstrate their agreement to participate in the study.

\subsubsection{Briefing and debriefing of participants}

All potential participants received a participant information sheet before being invited to be participate in the study. This described the main briefing points for participation in the interviews (a copy is provided in Appendix 3). The nature and key features of the study were discussed with the service user participant immediately prior to the interview as well as a preprepared prologue being read out emphasising confidentiality and voluntariness of participation (see prologue in Interview schedule - Phase 1 in Appendix 1). At the beginning of the interview, it was clearly stated to participants that the researcher had a duty of care to the participant and that if she / he became upset, the interview may need to be stopped.

Debriefing was a critical part of the research process. Debriefing was conducted at the end of the interview and involved a conversation with the participant. This discussion covered how the participant was feeling at the close of the interview, whether they required any emotional support, and whether they felt that they were happy to end the interview. Emotional support was provided after completion of the research interview so that this would not interfere with the integrity of the research by way of therapeutic misconception (Hewitt, 2007). The researcher sought to remain sensitive to evidence of distress or discomfort on behalf of the participant. If any issues or concerns became apparent during the debriefing process, this was communicated to available staff immediately.

Having described ethical issues concerning this piece of research, I now move onto describe specific methods I undertook for data collection and data analysis in the following two sections. 


\subsection{Method of data collection}

The study involved two phases. Phase 1 involved semi-structured interviews of service users with a history of SMI transiting between primary and secondary mental health care levels in West Wales. Phase 2 involved semi-structured interviews of managers, workers and volunteers in third sector organisations with experience of care and support of service users at the interface of primary and secondary mental healthcare levels in West Wales. Collecting data in two stages from two separate types of participants, service users and workers, allowed me to collect data generated from two main perspectives. This accorded with the perspectivist paradigm (Heaton et al., 2012; Rose et al., 2006; Sweeney et al., 2016) introduced in Chapter one, and the related interpretivist paradigm for social research of individuals, which I have elaborated in this chapter. This then is one example of how I based design features of method upon methodology and paradigmatic underpinning, in line with the tiered structure of Creswell and Plano Clarke's (2011) model.

\subsubsection{Participant recruitment and sampling strategy}

\subsubsection{Phase 1}

Male and female participants $(n=16)$, who had experienced mental health issues, were recruited through projects and networks which exist within the geographical area of West Wales for the purposes of engaging in semi-structured interviews. Inclusion criteria necessitated that participants had been discharged from secondary adult mental health services to primary care within the past three years. This meant that the research focus was on investigating people who had transited across the interface of secondary and primary care levels in Wales.

Participants for qualitative interviews in the first phase of research were recruited through a third sector umbrella organisation, West Wales Action for Mental Health (WWAMH; see www.wwamh.org.uk/), who facilitated access to a range of participating organisations providing day centre and support services to people with mental health difficulties. I met with a mental health development worker representing this organisation to discuss working with them for the purposes of this research project. It was agreed in this meeting that, in principle and subject to satisfactory ethics clearance, they would be happy to grant me access and 
permission to recruit participants through their various networks and projects. Participants, who had a broad variation of mental health issues, were recruited from a wide geographical area through these projects and networks. This was done by my visiting various centres and speaking about my research. Interested individuals were then assessed according to inclusion and exclusion criteria and the parameters of the sampling strategy.

A recruitment leaflet (see Appendix 6) was distributed throughout projects and networks associated with WWAMH. A poster (see Appendix 5) was also displayed in these areas. WWAMH offered the facility to distribute the leaflets and posters on the researcher's behalf and placed them in associated areas and organisations. Criteria for inclusion in the study, such as the need to have been discharged from secondary care within the last three years, were described on the leaflet and poster so that interested persons could be self-selecting. Contact details of the researcher were also provided on the poster and leaflet.

Interested persons were able to make further enquiries by contacting me or by speaking to staff working in WWAMH. Further information was then provided in the form of a participation information sheet (see Appendix 3). On the participant information sheet, it was requested that potential participants try to take up to fourteen days to consider whether or not they wish to be involved in this study. The amount of time they had taken to think about whether or not to participate was discussed once the interested person contacted me about being interviewed.

Consent forms were provided with the participant information sheet. On attending the interview, the participants returned the completed, signed and dated consent form to me. I was then able to check understanding and consent in person with the potential participant before commencing the interview. It was also stated in the participant information sheet that potential participants should not return consent forms to staff, as this would breach their anonymity. I instructed staff not to receive completed consent forms from any individuals.

A list of names and contact details of participants, consent forms, and appointment checklists were kept in a secure location (details about how these items will be securely stored has been provided in section 3.4 on ethical issues). These persons were then contacted and arrangements 
were made for them to attend an interview at a time which was convenient for them either at the main meeting room of WWAMH head office or at the location of the particular third sector organisation they attended. These details were then placed on an appointment checklist kept in the same secure location.

\subsubsection{Phase 2}

For Phase 2 of data collection, male and female participants $(n=16)$ were selected and recruited through my dissemination of the details of my project to third sector managers and at third sector staff meetings. In all cases, initial contacts had been made with staff through the first phase of my data collection, which involved regular journeys to locations in order to recruit participants. Professionals, clinicians and volunteers were recruited who had had experiences supporting people at the interface of primary and secondary mental healthcare in Wales. Managers were also recruited who had a range of experiences and oversight of the processes and systems whereby people transit between primary and secondary care services, and the effect this can have on care continuity and recovery. Three General Practitioners were recruited in this phase to be interviewed about their experiences of primary mental healthcare delivery for people with SMI who have been discharged from secondary services.

\subsubsection{Sampling strategy}

The sampling strategy was that of a purposive maximum variation approach to ensure heterogeneity of the sample. Purposive sampling is a form of non-probability sample in which participants are selected in a strategic way so that they are representative of the people who are the focus of the study (Bryman, 2016). Sample size tends to be much smaller for qualitative research compared to quantitative research since findings are developed inductively and validity is not based on deductive generalisations developed out of observations of a statistically representative section of the population (Bryman, 2016; Seale, 2011). Additionally, the time-consuming nature of in-depth qualitative analysis creates practical limitations on the quantity of data which may be used and properly analysed in qualitative research. The recommended number of interviews for qualitative research is between 12 and 60, with 30 being the mean (Adler and Adler, 2012). The combined number of interviews for Phases 1 and 2 was 32 interviews. I ensured that the number of participants was the same for both phases to 
provide a balance between service user and professional views, as I did not want to create an imbalance between the contributions of the two perspectives constituting the multi-perspectival epistemological paradigm of the research study (Rose, 2006; Sweeney, 2016). I particularly wanted to avoid having a greater number of participants who were professionals or volunteers in Phase 2 since service user views are often underrepresented in studies (Heaton 2012).

For Phase 1, individuals of both genders were selected with various mental health conditions and ages. A 'snowball' sampling strategy (Noy, 2008) was employed when the opportunity presented itself, in which word of mouth about potential participants at venues led to further recruitment. This occurred for instance in the case of finding and selecting GP participants. It appears that after the initial interview, a positive report was relayed amongst medical colleagues about me as a researcher undertaking an interesting project. Additional interested participants then contacted me whom I was able to recruit.

\subsubsection{Inclusion criteria}

Inclusion criteria did not specify that participants must have a specific type of mental health diagnosis since psychiatric diagnosis can be imprecise, may change over time for an individual person, and may be difficult to confirm (Hyman, 2010; Rogers and Pilgrim, 2014). It is not therefore seen as a helpful or reliable indicator for my inclusion criteria. Since there is a very close association between being treated in secondary mental health services and a diagnosis of SMI, having received this kind of treatment was considered a practical, valid and reliable inclusion criterion for selecting people with SMI.

\section{Summary of inclusion criteria for Phase 1:}

- Participants will have been discharged from secondary adult mental health services to primary care within the past three years.

- Participants must be between the age of 18 and 65 in order that the target population falls within the category of adult mental health. 


\section{Summary of inclusion criteria for Phase 2:}

- Participants will be workers in third sector organisations providing support for people with SMI including those who have been discharged from secondary services.

- Participants will be volunteers for third sector organisations who are also peer support workers.

- Participants will also include General Practitioners.

\subsubsection{Exclusion criteria}

Exclusion criteria for both phases applied to people identified as being unwell and unable to participate. There were no further exclusion criteria relating to type or duration of mental health problem since this exploratory study was aiming for maximum variation and heterogeneity in the characteristics of persons making up the sample.

\section{Summary of exclusion criteria for Phases 1 and 2:}

- People who are currently in acute mental health crisis for whom there are doubts about their capacity to give informed consent. Since capacity may fluctuate, this may occur at the time of interview.

\subsubsection{Interviews}

According to the methodological paradigm of constructionism, the subject matter of the interview is collaboratively produced by both interviewer and participant (Gubrium and Holstein, 2004; Silverman, 2014). The interviewee is not a passive vessel waiting to be tapped, to whom the researcher 'gives voice' so that he or she may provide an account of events (Fine, 2002; Gubrium and Holstein, 2004). Within social constructionism, the social world is manufactured through human interpretation, interaction and language which constitute the interview. The interviewer also plays an important role in the interactive production of meaning. Since the interviewer exercises a degree of control over the flow and direction of the interview, he or she plays an active role in producing the meanings which are constructed 
during the course of the interview (Rapley, 2004). Consequently, the researcher must practice reflexivity (Coffey and Atkinson, 1996; Delanty and Strydom, 2003).

This constructionist understanding of interviewing should be qualified by the aspect of realism I have introduced in adopting a contextualist constructionism. In relation to interviews, this version of constructionism designates that they will connect with an element of objective reality and that different accounts may therefore entail varying degrees of validity. Hammersley (2008) supports this view as follows: "The fact that interview accounts are always constructions [does not] mean that they cannot be accurate representations; any more than the fact that research reports are constructions rules out their claim to represent the world" (p.10). However, at the same time, I do not consider that interviews allow for a direct accessing of facts or events in the manner of a naïve realist epistemology. People cannot always be relied upon to report information accurately, and discrepancies between objective measures and subjective reports have been well documented (Kitzinger, 2004). Therefore, I considered the content of interviews more essentially to be 'speech acts' than representations, providing an indirect representation of experienced events and facts, influenced by specific agendas in performing the speech act, and coloured by the unique perspective, cultural and social background which the participant possesses (Atkinson et al., 2003; Austin, 1975; Coffey and Atkinson, 1996; Goffman, 1959; Lyman and Scott, 1970; Searle, 1979). I considered this to be consistent with my methodological underpinning of contextualist constructionism incorporating critical realism. It also ties in with the specific approach of discourse analysis I employed, which I will introduce in section 3.6.4.

I chose to conduct semi-structured interviews as opposed to unstructured or structured interviews, as they are particularly suited to the exploration of experiences and opinions of participants regarding complex and sensitive issues which may form the basis of meaningful constructions (Barribal, 1994). Prompts and probes were used to guide a free-flowing interview where the participants took the lead in describing experiences related to the research focus. This was intended to emphasise the experiential aspect of participants' accounts and encourage the creative and interactive stimulus for constructed meanings. 


\subsubsection{Question formation and interview schedule}

Question formation for semi-structured interviews, including prompts and probes, was based on issues generated by the evidence synthesis of the literature review covering care continuity, recovery and primary mental healthcare implementation in Wales. A series of open questions were developed with the intention that they would stimulate an informative and free-flowing discussion remaining relevant to the research focus. The interview schedule for the second phase was largely based on the first schedule, although wording was altered since participants were no longer talking about their own experiences of care continuity. I also drafted versions of both interview schedules for my supervisors to comment on so that their expertise could contribute to developing the wording and sequencing of questions, and add a level of face validity to the design of the inquiry. Copies of both interview schedules are provided in Appendices 1 and 2.

\subsubsection{Recording and transcribing of interviews}

I used digital audio recording equipment to record interviews. These were later transcribed in full, partly by me ( $n=8$ transcripts in Phase 1 and $n=7$ transcripts in Phase 2) and partly by a commercial interview transcribing service, www.uktranscription.com, whose services I hired ( $n=8$ transcripts in Phase 1 and $n=9$ transcripts in Phase 2). I describe the method of transcription more fully in section 3.6 on data analysis. Although, transcription might be regarded as data collection and not data analysis (Antaki et al., 2003), I aligned myself with Braun and Clarke's (2006) understanding which places the transcribing process in the first phase of analysis. The view that transcription is a preliminary stage of analysis is based on the notion that it involves some interpretation on behalf of the transcriber and initial familiarisation with the data (Bird, 2005; Lapadat and Lindsey, 1999; Riessman, 1993). Within the constructionist paradigm therefore, I regarded transcription as the beginning of the meaningmaking process (Burr, 2003; Jørgensen and Phillips, 2002) in which activities of understanding and interpretation have already begun to occur. Since I did not transcribe some of the interviews, I compensated for this omission of meaning-making activity by reading and rereading these transcripts an additional number of times after they were sent to me. 


\subsection{Method of data analysis}

The approach I adopted for analysis of interview transcripts was thematic analysis (Boyatzis, 1998; Braun and Clarke, 2006; Braun et al., 2014; Rivas, 2011) incorporating discourse analysis (Edwards and Potter, 1992; Fairclough, 2003; Gee, 2004; Jørgensen and Phillips, 2002). The combination of these two approaches is called 'thematic discourse analysis' by Singer and Hunter (1999). I employed the six-phase approach to thematic analysis described by Braun and Clarke (2006) incorporating discourse analysis into the final phases to form my approach of thematic discourse analysis. In this section, I shall first describe the phases of Braun and Clarke's (2006) approach to thematic analysis, then detail how I introduced discourse analysis at the end of this process in order to generate final themes for the findings chapters.

Thematic analysis is a flexible and accessible method for analysing and identifying patterns or themes within qualitative data that is not necessarily committed to a particular theoretical or epistemological position (Braun and Clarke, 2006). However, it may be situated within a theoretical position such as the contextualist constructionism and discourse theory which I employ. Thematic analysis is not seen as a specific method in its own right by some researchers, but rather as a process which consistently occurs across different qualitative analytical approaches (Boyatzis, 1998; Ryan and Bernard, 2000). Braun and Clarke (2006) have however pioneered the view that thematic analysis can be a method employed in its own right. Although I agree with this innovation, and adopted Braun and Clarke's (2006) approach, I concluded it would add rigour to ally it with a major analytic tradition, which I chose to be discourse analysis. I felt this added rigour would contribute greater credibility to findings and generated themes. I also considered thematic analysis to be an appropriate methodological approach for the research design and focus because of its flexibility and accessibility, its ability to generate unanticipated insights, and its capacity to cover a large data-set with a rich thematic description (Braun and Clarke, 2006; Ryan and Bernard, 2003).

Braun and Clarke (2006; see also Braun et al., 2014) divide the process of thematic analysis into six phases which are summarised in Table 3. I followed each of these phases as I carried out my own thematic analysis of interviews. The following sections describing the procedure 
of my data analysis follows the structure of these phases. Discourse analytical approaches were introduced in the final phases, five and six.

\section{Table 3: Phases of thematic analysis}

\begin{tabular}{|l|l|}
\hline Phase & Description of the process \\
\hline $\begin{array}{l}\text { 1. Familiarising yourself } \\
\text { with your data }\end{array}$ & $\begin{array}{l}\text { Transcribing data, reading and re-reading the data, noting } \\
\text { down initial ideas. }\end{array}$ \\
\hline 2. Generating initial codes & $\begin{array}{l}\text { Coding interesting features of the data in a systematic fashion } \\
\text { across the entire data set, collating data relevant to each code. }\end{array}$ \\
\hline 3. Searching for themes & $\begin{array}{l}\text { Collating codes into potential themes, gathering all data } \\
\text { relevant to each potential theme. }\end{array}$ \\
\hline 4. Reviewing themes & $\begin{array}{l}\text { Checking if the themes work in relation to the coded extracts } \\
\text { (Level 1) and the entire data set (Level 2), generating a } \\
\text { thematic 'map' of the analysis. }\end{array}$ \\
\hline 5. Defining and naming \\
themes & $\begin{array}{l}\text { Ongoing analysis to refine the specifics of each theme, and } \\
\text { the overall story the analysis tells, generating clear } \\
\text { definitions and names for each theme. }\end{array}$ \\
\hline 6. Producing the report & $\begin{array}{l}\text { The final opportunity for analysis. Selection of vivid, } \\
\text { compelling extract examples, final analysis of selected } \\
\text { extracts, relating back of the analysis to the research question } \\
\text { and literature, producing a scholarly report of the analysis. }\end{array}$ \\
\hline
\end{tabular}

(Braun \& Clarke, 2006, p.86)

\subsubsection{Phase 1: Familiarisation with data}

As I described in section 3.5.3.2, transcription was an initial part of the process of familiarising myself with the data. This was the first point at which I encountered my entire data set in a meaningful way. I shall therefore commence this section with a summary of my method of transcription.

Audio recordings of semi-structured interviews were transcribed partly by me and partly by a transcribing service (www.UKTranscription.co.uk). Transcribing conventions provided by Silverman (2014) were used (see Appendix 8), and to a greater extent than would normally be required by thematic analysis alone because of my incorporation of discourse analysis into this 
approach. I used a transcribing service due to the volume of data needing to be transcribed and my desire to code and analyse the data at as early a stage as possible. To compensate for the loss of the initial opportunity to familiarise myself with data by transcribing these recordings myself, I read and re-read these transcripts an additional number of times after they were sent to me. I also checked these transcripts for accuracy by listening to stretches of the audio recordings in combination with reading them. During the initial transcription or checking of transcriptions phase, I wrote notes and memos on my initial thoughts in response to the data. This further contributed to my approach in which transcribing was an important initial stage of the interpretative process within qualitative analysis.

I continued to read and re-read transcripts after they had been transcribed in order to immerse myself fully in the data (Braun and Clarke, 2006; Braun et al., 2014; Boyatzis, 1998). During this stage of familiarisation with and immersion in the data set, I continued to write memos and theoretical notes about my ideas and reflections as they occurred to me (Ryan and Bernard, 2000). These memos were a continuation of the field notes and reflexive diary which I had kept whilst collecting data and conducting interviews. Writing memos and theoretical notes was also the beginning of my generating codes and concepts about the data.

\subsubsection{Phase 2: Generating initial codes}

I considered coding to be a central part of the analytic process since coding prompts the researcher to make judgments about the meanings of portions of text (Coffey and Atkinson, 1996; Ryan and Bernard, 2000). In view of this, I endeavoured to conduct my coding according the contextual constructionist paradigm underlying this work, where codes could initiate the process of identifying intransitive structures of the social world underlying constructions (see sections 3.3.4 and 3.3.6). The distinction between sociologically constructed codes and surface level, in vivo codes made by Strauss (1987), was helpful in this respect, and informed the code making process. I also attempted to combine inductive and deductive approaches in different stages of coding in order to discern this 'deeper' sociological structure (Rivas, 2011). Practically speaking, this meant that I attempted to code everything that was meaningful in a general sense (in vivo codes), and chunks of text that appeared already to be meaningful in relation to the theory and healthcare research that I was undertaking (sociologically constructed 
codes). In this way I initiated the process of developing constructions which would generate new knowledge for the research study.

Coding was carried out in three distinct stages. In the first stage of initial or preliminary coding I developed an initial semantic sketch of the data set whereby I tagged significant portions of the text using the comments facility of the Microsoft Office Word application. I saw this stage of preliminary coding as an extension of the development of memos and theoretical ideas I carried out in the transcription and initial reading and re-reading stages of data analysis. I continued this process of initial coding throughout the data set to ensure that all data had received this initial analytic treatment.

Having completed this initial stage of coding built upon original memos and theoretical ideas, I proceeded to a more inductive and systematic stage of 'open coding' (Corbin and Strauss, 2008; Glaser and Strauss, 1967). In this stage I ensured that each part of the data was treated in the same way, and coded whether or not the code was relevant to my research question (Rivas, 2011). Coding systematically in this manner was important since this contributes greatly to rigour of the analytical approach and consequently to the validity of themes and findings based on the analysis (Coffey and Atkinson, 1996).

The third stage of coding was 'intentional' or 'selective' coding. Implementing this stage of coding follows the progression from general to intentional coding described by Coffey and Atkinson (1996). In this stage, I identified and coded sections of text which were meaningful in relation to my research inquiry into care continuity and health.

It should be noted that I did not use conventional software such as NVivo or MAXQDA to perform coding. Initially, I did start to use NVivo but found this application to be fairly cumbersome and unwieldy for my purposes in coding the data set as a preparatory stage for indepth analysis. Ultimately, I decided upon an approach which combined manual coding of transcripts with the use of concept mapping software (http://compendium.open.ac.uk/). Combining manual and electronic approaches in this way enabled me to organise my manual codes with the efficiency and scope of a computer application, but with the benefits and 
flexibility which is afforded by a manual approach to coding. It also provided the basis for easily collating codes into concepts, since I was using a piece of software specifically designed for concept mapping to do the coding in the first place. This put me in good stead for my approach to the later phases of Braun and Clarke's (2006) method, which was based on generating concept maps and then subjecting data extracts identified in these taxonomies to discourse analysis (see next two sections).

\subsubsection{Phases 3 - 6: Identifying and reporting themes}

Having generated initial codes across the 32 transcripts, I then proceeded through the final four phases of Braun and Clarke's (2006) method of thematic analysis. Braun and Clarke (2006) provide guidance for these phases involving collating codes into candidate themes at Phase 3 , generating a thematic map at Phase 4, refining and defining final themes at Phase 5, and at Phase 6, producing a final report containing vivid, compelling extracts with a theoretical application of relevant literature (Braun and Clarke, 2006; Braun et al., 2014). I followed this guidance generally speaking but my actual method was quite precise in terms of how I carried out the objectives of these phases, because I employed the specific approaches of concept mapping and discourse analysis within these phases. Phase 3 therefore in my methodology was the process of collating the many codes distributed throughout the transcripts into concepts made up of similar codes. For instance, I collated $(n=23)$ codes distributed amongst $(n=5)$ participants for the concept 'Lack of mental health training' (see Figure 12 below) referring to the perception talked about in different ways in participants' talk that GPs were not sufficiently trained to provide mental health treatment. I continued to collate codes in this way until I had built a large, initial concept map for the entire data set, corresponding roughly to Braun and Clarke's (2006) Phase 4. The key difference in my approach was that I did not generate a thematic map of candidate themes at these two phases. Rather, I generated a concept map as an inductive approach constituting a preparatory stage for generation of themes.

Concept maps were developed first for individual participants and then these were combined together to assemble a large concept map for the entire data set. An example of an individual participant concept map is provided in Appendix 10. I therefore combined within-case and across-case conceptual analysis (Ayres et al., 2003) in a systematic and inductive manner which 
involved simply merging the concept maps of individual participants into a single large concept map for all participants. I then worked my way through this concept map for the entire data set, refining this taxonomy, removing duplication and combining concepts together where this seemed appropriate. The end result of this, which I considered to correspond roughly to Phases 4 and 5 of Braun and Clarke's (2006) approach, was the generation of refined concept maps representing the entire data set. Categories were designated as the main concepts within each concept map. I provide figures of all categories and associated concept maps throughout Chapters four to six in the context of a description of my analysis and findings.

Generation of themes was then carried out on the basis of discourse analysis of key data extracts located in the data set, whose selection was guided by the conceptual analysis presented in the refined concept maps. Crucially, I used the concept maps as a guide to help me navigate through the large data set and accurately identify key passages of talk suitable for discourse analysis, which were relevant to the concept being considered or argument being developed. Themes were then generated both by consideration of the contextual information for data extracts provided by the concept maps, and also by detailed, in-depth qualitative analysis of participants' talk which was carried out through discourse analysis.

It was clear to me that inductive conceptual analysis which produced the concept map for the entire data set should not be sufficient for me to perform thematic analysis. Antaki and colleagues (2003) have indicated that merely doing an inductive, conceptual analysis or other type of summarisation of data does not amount to true qualitative analysis of people's talk. They class this error as under-analysis through summary. However, the extensive process of concept mapping did play an important preparatory role for me to perform discourse analysis which was the consummating process enabling me to generate themes. It allowed me to connect with the entire data set and also acted as a tool for identifying and locating relevant passages of talk which were most suitable for discourse analysis. It was also clearly the case that I could not perform detailed discourse analysis of the entire data set. This would neither be feasible, nor would it be thematic discourse analysis in the sense that it is referred to by Singer and Hunter (1999) or Braun and Clarke (2006). My approach to thematic discourse analysis therefore combined extensive and comprehensive concept mapping with discourse analysis to generate themes. This approach provided an analytical context for discourse analysis of data 
extracts. This combined approach to qualitative analysis offered both comprehensiveness and fidelity, ensuring the highest quality methodological approach contributing to theme construction.

Roughly speaking, the final stage of discourse analysis, building on top of conceptual analysis, can be located within Phases 5 and 6 of Braun and Clarke's (2006) method, where themes are identified and named with a theoretical application of relevant literature. This stage is detailed throughout the three findings chapters from Chapter four to Chapter six. The writing up activity in Phase 6 was actually an important part of analysis for me. I found that I could only truly carry out discourse analysis and subsequent thematic analysis through writing about it, and conveying my thoughts, insights and arguments about the ways in which participants' talk was functioning. Analysis was then developed as an iterative process drawing on feedback from my supervisor as I composed a series of drafts describing my developing thematic discourse analysis. Writing was therefore a key part of analysis, and this process of representation was a key part of the process of constructing themes. As Coffey and Atkinson (1996) put it, "We reconstruct culture through our own acts of representation" (p.137), meaning that representation, which for me culminated in writing about my discourse analysis, is a key way in which knowledge and themes are constructed. In the next section, I describe the approach I used to perform discourse analysis and how this led to generation of themes in the context of conceptual analysis.

\subsubsection{Discourse analysis: an integrated approach}

Discourse analysis focuses on the key role that language plays in the social construction of meaning (Burr, 2003). This accords with the constructionist paradigm underpinning my work, in which language is a precondition for thought and reality, rather than arising as a consequence of thought and reality (Burr, 2003). For this reason, discourse analysis is one of the most widely used approaches within constructionism (Jørgensen and Phillips, 2002). This contributed to my decision to employ discourse analysis in my method of qualitative analysis.

Discourse analysis is a heterogeneous association of theoretical perspectives and approaches within qualitative, sociological analysis of language in the form of 'discourse' - i.e. talk and 
text (Burr, 2003; Edwards and Potter, 1992; Silverman, 2014). These approaches include a variety of methods such as critical discourse analysis, conversation analysis and discursive psychology (Antaki et al., 2003; Edwards and Potter; 1992). I chose to employ an approach combining critical discourse analysis (Fairclough, 1992b; 2003; 2013) and discursive psychology (Antaki and Widdicombe, 2008; Edwards and Potter, 1992; Wiggins, 2017) with some influence from the approach of Laclau and Mouffe (1985). My method was therefore integrative, utilising the distinct but complementary resources available in these separate approaches. This was not an eclectic approach involving a mishmash of disparate approaches since I considered the theoretical relations between them and how these may be aligned to form an integrative approach, which I now outline (Jørgensen and Philips, 2002).

Combining these three different approaches to discourse analysis allowed me to make use of their individual analytical strengths whilst compensating for their weaknesses. For example, critical discourse analysis provides a theoretically strong explanation for the position of a discourse within a wider context of discourses and society, and how this is related to power relations between individuals and groups (Fairclough, 1992a; 1995; 2003). Despite this strength, critical discourse analysis is weak in accounting for human agency, which may be considered to be a side-product of its tendency towards a post-structuralist emphasis on linguistic structuration, and its indebtedness to Foucauldian analysis (Burr, 2003; Foucault, 1972; Willig, 2013). Critical discourse analysis also lacks the empirical concepts available in discursive psychology to describe the linguistic tools used by human agents to construct discourses, perform 'speech acts' with language and position themselves in relation to talk (Austin, 1975; Burr, 2003; Davies, 1990). However, a weakness of discursive psychology is that it tends to be less informative about the implications which discourses have for power imbalances between people, and broader objective features of the social world (Jørgensen and Philips, 2002).

I therefore sought an integrative method for performing discourse analysis, where the weaknesses and deficiencies of one approach would be compensated for by the strengths and resources of another. For instance, I wanted to be able to understand how participants' talk was used in an immediate way to negotiate representations of the world and identities-in-talk, an understanding which could be provided by discursive psychology (Antaki and Widdicombe, 
2008; Jørgensen and Phillips, 2002; Wiggins, 2017). At the same time, I wanted to be able to understand how discourses might have wider implications for the social world and power relations in the area of healthcare I was researching. In addition to critical discourse analysis, Laclau and Mouffe's (1985) discourse analysis also has the potential to elucidate these broader areas of social inquiry. They provide a highly poststructuralist theory of the fundamental instability of language, in which meaning can never be permanently fixed. Consequently, different discourses of the social world are engaged in a constant discursive struggle to achieve hegemony by fixing the meanings of language in their own way (Jørgensen and Phillips, 2002; Laclau and Mouffe, 1985).

To make sense of the way in which these approaches and analytical resources could be combined in a coherent way, I drew on Sally Wiggins' (2017) use of the metaphor of a lens being used by a documentary maker to describe different approaches within discourse analysis. A fish-eye or wide-angle lens on discourse and society is provided by critical discourse analysis or Laclau and Mouffe's (1985) discourse theory, whilst a zoom lens is provided by discursive psychology. I therefore decided to combine the approaches of critical discourse analysis, Laclau and Mouffe's (1985) approach and discursive psychology, drawing on their different strengths, analytical resources and full range of perspectives on the social world, for the purposes of performing my investigation of participants' experiences of care continuity and recovery.

Potter (1997) argues that a 'recipe' approach such as the one I have outlined here, combining different methods into an integrative whole, is inappropriate for the activity of discourse analysis which may better be regarded as a craft skill not reducible to a set of methodological features. However, McLeod (2001) counters by arguing that obfuscating the 'how to' aspect of analysis has the potential to keep methods mysterious which may be tantamount to a form of elitism. I found myself persuaded by this latter argument and felt I was on firmer ground where I could draw on articulated methods and approaches for performing discourse analysis. Therefore, I employed 'how to' approaches provided by Wiggins (2017), Gee (2010) and Fairclough (2003), which were the basis for my analysis. I was also concerned that I would produce genuine analysis, and not fall into any of the pitfalls of discourse analysis identified by Antaki and associates (2003). One way that I achieved this was to constantly try to 
understand how discursive devices were being used by participants in their talk to construct and negotiate meanings and position themselves or others in their discourse, rather than simply identifying discursive devices in talk in a mechanistic way. Additionally, I found that performing discourse analysis was very much a state of mind and attitude towards data extracts of talk. It was helpful for me to distance myself or defamiliarise myself from the talk I was analysing, an approach referred to by James Paul Gee (2010) as "making strange" (p.11). Wood and Kroger (2000) supplement this understanding with their advice that focusing on the literal meaning of talk or text is the least helpful strategy in performing discourse analysis. I recognised that what underlies this counterintuitive state of affairs is the realisation that the analyst is part of the culture he or she is analysing. Therefore, many of the discursive devices employed will be normalised for the analyst and difficult to detect, embedded within commonsense understandings which produce the more obvious and literal meanings of talk or text. These common-sensical understandings also function to perpetuate hegemonic fixings of meaning which may delimit the horizons of the perspective on discourse and society in which the researcher is embedded (Crossley, 2005; Gramsci, 1971; Kincheloe and McLaren, 1994; Laclau and Mouffe, 1985). It was my job therefore as an analyst to step outside of the various functions and actions achieved discursively in talk which contribute to its literal meaning, in order to achieve an in-depth understanding of the constructed and situated nature of discourse within wider society.

My approach of discourse analysis which I have described in this section was therefore the stage at which themes could be properly identified, corresponding to Phase 5 of Braun and Clarke's (2006) six phase methodology. They describe a theme as capturing "something important about the data in relation to the research question, and represent[-ing] some level of patterned response or meaning within the data set" (Braun and Clarke, 2006, p.82). Since the research question was about the care continuity and recovery experiences of participants, themes generated in this study should capture key aspects of these experiences, according to Braun and Clarke's (2006) definition. In my view, this experiential and perspectival dimension could best be captured through discourse analysis of data extracts, which would elucidate the constructed and positional nature of participants' talk. The introduction of discourse analysis into my qualitative approach was therefore the key to illuminating the interpretivist dimension of the data set. This part of analysis was therefore the point at which themes could be generated, 
capturing the important meanings in talk related to the research question, in line with Braun and Clarke's understanding.

\subsection{Credibility of qualitative findings: quality, rigour and validity}

This final section covers the credibility of my qualitative research findings, and the issues of rigour, validity and quality criteria. A number of difficulties face attempts to establish the truthfulness and validity of qualitative research, including my own. Qualitative research approaches are often referred to as 'soft' science in contrast with quantitative approaches, referred to as 'hard' science (Guba and Lincoln, 1994). The use of the term 'hard' as opposed to 'soft' carries connotations of quantitative approaches having a more robust methodology and more valid epistemological product than is the case with qualitative research. Indeed, qualitative research has often been described as soft, sentimental, unclear and likely to lead to conclusions that have a questionable epistemic value (Bishop, 2007; Ritchie et al., 2013).

The difficulty in establishing the validity of findings with qualitative research is related to the difficulties and criticisms associated with the interpretivist epistemology on which it is based. In this vein, the findings of qualitative research are often accused of being impressionistic and overly-subjective (Bryman, 2016). Related difficulties occur in establishing reliability, replicability and generalisation of qualitative findings (Seale, 2011). Since notions of validity and reliability are rooted in the realist tradition underpinning quantitative research, their appropriateness for qualitative inquiry has been brought into question (Seale, 2011). The qualitative researcher may graft reliability and validity criteria from quantitative research into the qualitative arena, but this seems inappropriate given the interpretivist epistemology associated with qualitative research. Nevertheless, this approach is advocated by researchers such as LeComte and Goetz (1982) and Kirk and Miller (1986). Alternatively, the qualitative researcher may embrace different criteria for the validity of qualitative findings, based on standards of quality and rigour (Bryman, 2016; Seale, 2011). I decided to take the latter approach since I felt that it would be inconsistent with the interpretivist, constructionist outlook of my research if I were to use validity criteria sourced within quantitative research methodologies, and systems of epistemology rooted in a positivist paradigm. 
In order to implement standards of quality and rigour contributing to the validity of qualitative findings, I drew upon Sarah Tracy (2010) in her eight 'big-tent' criteria for excellent qualitative research. I thought that this was the most comprehensive and compelling account of quality and rigour criteria for qualitative research. These are worthy topic, generalisability or resonance and evocativeness of findings, rich rigour (rigour of approach), sincerity (reflexivity and transparency), credibility, significant contribution, high standards of research ethics and meaningful coherence (between research aim, methodology and background literature). It is these various qualities which I have sought to achieve throughout the research project. In the next section, I focus on a key criteria taken from this list concerning generalisability of findings.

\subsubsection{Theoretical generalisation}

Mitchell (1983) has described the manner in which qualitative research contributes new knowledge as 'theoretical generalisation'. This is contrasted with 'empirical generalisation' which is the deductive approach typically found in quantitative studies (Mitchell, 1983). Whilst quantitative research uses a deductive approach to test a proposed theory or hypothesis, qualitative research works upwards inductively from raw data to generate new theory. In this respect, quantitative research is sometimes described as a 'top-down' process where hypotheses are tested against observations. Qualitative research, on the other hand, is described as a 'bottom-up' process in which patterns and theory are derived from analysis of observations and data (Ritchie et al., 2013).

This 'bottom-up' inductive process is referred to by some qualitative researchers as 'theorising' an area of inquiry, where new theory is generated from the data through the theoretical sensitivity of the researcher, by placing the finding in its relevant theoretical context (Rivas, 2011; Seale, 2011). Mitchell's (1983) concept of validity based on 'theoretical generalisation' is one understanding of such inductive theorising in qualitative analysis. With this approach, it is the quality of theoretical inferences which is crucial to the assessment of generalisability and validity of findings in qualitative research (Bryman, 2016). Mitchell describes this as the "cogency of the theoretical reasoning" (Mitchell, 1983, p.207) which is the basis of validity in qualitative research. Theoretical generalisation is also described as a form of 'theory-building' where "conclusions are drawn from features or constructs developed in a 'local' or single study 
which are then utilised in developing wider theory which is or can be applied to other settings" (Ritchie et al., 2013, pp.38-349). ${ }^{8}$

I concluded that Mitchell's concept of 'theoretical generalisation' should constitute the central concept in my own determination of generalisability of findings in my study, because of the advantages I could see with this approach over other potential options for qualitative research such as inferential generalisation. Theoretical generalisation may be distinguished from inferential generalisation which is employed in many qualitative studies (Lincoln and Guba, 1985). Here, findings from a study are inferred to other settings or contexts. Context and setting are prerequisites for inferential generalisation meaning that principles deduced from findings may only be applied to other settings which bear some similarity to the original study context. Theoretical generalisation, on the other hand, is less limited by caveats of context and setting since it attempts to describe underlying ontological features of the social world, which may be elucidated through qualitative analysis (Ritchie et al., 2013). Arguments developed on this basis have broad applicability because they characterise the ontological fabric of the social world, which is discoverable through qualitative analysis of participants' talk, conducted according to a rigorous methodology. Since suppositions are more fundamental, conclusions gained through this process of theoretical generalisation have potentially wider applicability. I also considered that the element of critical realism philosophically underpinning my research would add a greater degree of objective validity to findings established by theoretical generalisation. For these reasons, I decided to adopt theoretical generalisation as a key contributor to validity of qualitative findings, which are developed in subsequent findings chapters and the discussion chapter.

\subsubsection{Theoretical generalisation and grounded theory}

The approach of theoretical generalisation has been developed primarily in relation to grounded theory (Corbin and Strauss, 2008; Glaser and Strauss, 1967; Strauss and Corbin 1990). However, the approach to theoretical generalisation I employ is not tied to the underlying assumptions of grounded theory. Instead, my approach is based on the one described by Seale

\footnotetext{
${ }^{8}$ Theoretical generalisation is also referred to as analytic generalisation in some literature (Polit and Beck, 2010; Smaling, 2003) though I will use the former term in this thesis.
} 
(1999), where a theoretically broad, cohesive understanding is advanced without incorporating the notion that a complete theoretical analysis of research phenomena, or a substantive theoretical explanation has been reached (Corbin and Strauss, 2008; Strauss and Corbin 1990).

One of the key tenets of grounded theory is the idea of theoretical saturation (Glaser and Strauss, 1967; Seale, 1999). According to this understanding, theoretical generalisation is based on "the discovery of theory from data systematically obtained from social research" (Glaser and Strauss, 1967, p.2). This process is continued until the point of data saturation or theoretical saturation is reached (Corbin and Strauss, 2008; Glaser and Strauss, 1967). At this point, further data collection and analysis apparently fails to reveal new information, meanings or patterns within the data. The researcher concludes on this basis that she has developed a complete theoretical analysis of the research phenomena, and that no further explanation is necessary or possible. This comprehensive understanding is referred to as a substantive theoretical explanation (Corbin and Strauss, 2008; Glaser and Strauss 1967; Strauss and Corbin 1990).

However, the notion of theoretical saturation is criticised for being a nebulous concept with no agreed consensus on how it may be achieved or defined (Bowen, 2008). Perhaps a more critical shortcoming from my point of view is the implication that phenomena may be comprehensively understood in the form of a substantive explanation produced once the point of theoretical saturation has been reached (Dey, 1999). This would seem to contradict the interpretivist philosophical underpinning I described in section 3.3 rooted in the neo-Kantian view that phenomena can never be comprehensively known as 'things-in-themselves' (Manicas, 2006; Protevi, 2005). The constructionist and interpretivist paradigms which underpin this research are anathema to the idea that a point may be reached where phenomena may be comprehensively known or understood. In section 3.3, I summarised this philosophical outlook with the existentialist concept of 'intentionality', which I felt captured the sense of there being a "radical interdependence of subject and the world" (Crotty, 1998, p.45). This was also the basis for the particular understanding of social constructionism which I have provided in this methodology chapter to underpin this piece of research. Consequently, in order to accord with the philosophical underpinning of my methodology, I decided to reject the notion of theoretical saturation and the possibility of reaching a substantive theoretical explanation. 
The implications of this position on theoretical generalisation for findings is that there exists an unlimited potential for new meanings and interpretations to be generated about research phenomena. The meaning-making process which generates findings should not limited to the finite span of theoretical saturation held within a particular piece of research, along with its analysis and theory-building. Therefore, conclusions may always be refined or even refuted through further empirical investigation, analysis and exploration of phenomena. There will always be room for further understanding and insight yielded from the appropriate application of another theoretical context or understanding. It might be perfectly possible therefore for another researcher to generate entirely new theoretical interpretations of my data set, or to expand upon my findings.

\subsubsection{The relationship between discourse and the social world}

My adoption of thematic discourse analysis has further implications for qualitative generalisation. There are a variety of different views on the relationship between discourse and the world within discourse theories, which has implications for the way in which analysis and findings in this research study might be generalisable to provide new knowledge about the wider social world. The various viewpoints amongst theorists on the role of discourse in the constitution of the world have been summarised by Jørgensen and Phillips (2002) in the following figure:

Figure 4: The role of discourse in the constitution of the world

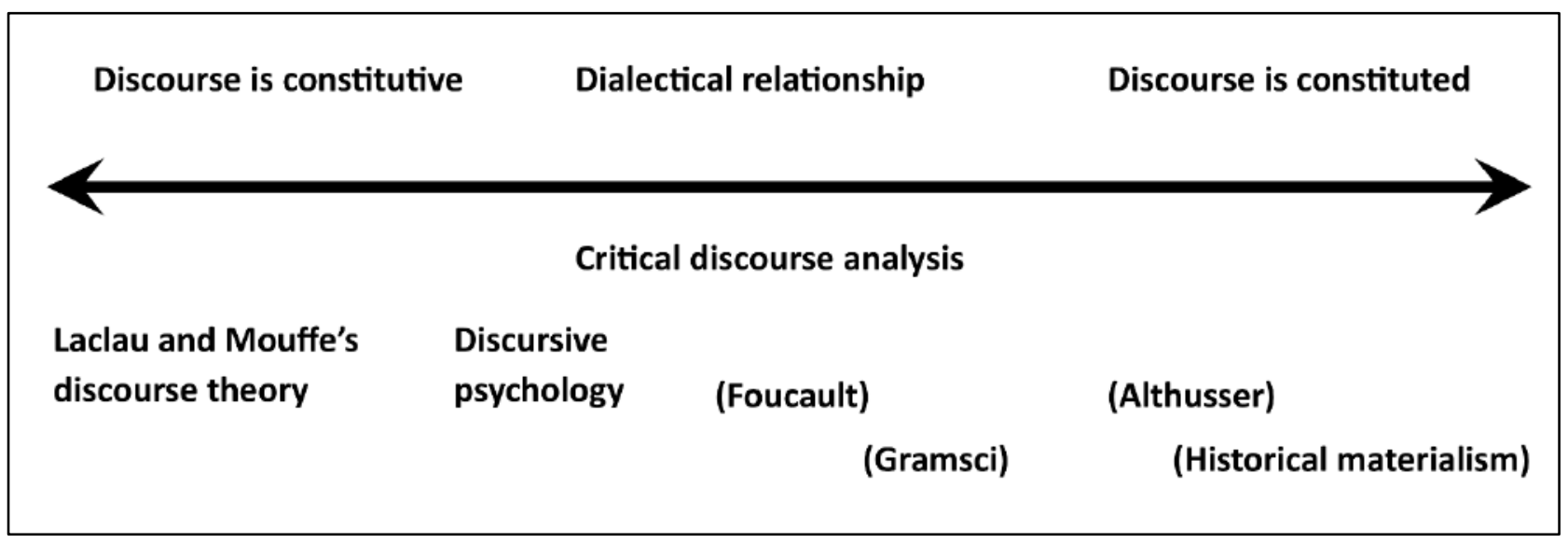

(Jørgensen and Phillips, 2002, p.20) 
In this figure, a spectrum exists between two polarities. At the one extreme is the view that the social world is fully constituted by discourse. This is the highly poststructuralist view held by Laclau and Mouffe (1985), based on the notion that discourse constructs the social world in meaning which is unstable because of the fundamental instability of language (Jørgensen and Phillips, 2002). At the other extreme is the opposing view that discourse is constituted by the social world. This side of the spectrum is occupied by the Marxist, historical-materialist theories of Althusser (1971) and Gramsci (1971). These Marxist-oriented positions view discourses as mere reflections of more fundamental social mechanisms such as the economy and market forces (Jørgensen and Phillips, 2002). Fairclough's critical discourse theory (2013) is located in the middle of this spectrum. According to this view, discourse, though important, is not fully constitutive of the social world (Fairclough, 1992a). Rather, it constitutes one dimension of social practice. Discursive psychology occupies a similar position to critical discourse theory in the above figure. However, as was discussed in section 3.6.4, it should be recognised that discursive psychology has a limited capacity to describe or determine broader objective features of the social world (Jørgensen and Phillips, 2002). This impedes the suitability of this approach to contribute to theoretical generalisation in this thesis, despite the substantial role that it has in later analysis clarifying the linguistic tools used by participants to construct discourses.

Focusing on the poststructuralist view held by Laclau and Mouffe (1985) that the social world is fully constituted by discourse, they argue that there is no distinction between discursive and non-discursive dimensions of the social world. Instead, all phenomena within the social world are discursive entities:

"Our analysis rejects the distinction between discursive and non-discursive practices. It affirms: a) that every object is constituted as an object of discourse, insofar as no object is given outside every discursive condition of emergence; and $b$ ) that any distinction between what are usually called the linguistic and behaviourial aspects of a social practice, is either an incorrect distinction or ought to find its place as a differentiation within the social production of meaning, which is structured under the form of discursive totalities."

(Laclau and Mouffe, 2001, p.107)

A distinction between discursive and non-discursive elements is seen as an "incorrect distinction", which reflects the false dichotomy recognised in section 3.3 between subjective 
and objective planes. Laclau and Mouffe (1985) go on to emphasise that this position, which I will label 'discursive universalism', is not an anti-realist stance:

"The fact that every object is constituted as an object of discourse has nothing to do with whether there is a world external to thought, or with the realism / idealism opposition."

(Laclau and Mouffe, 1985, p.108)

Laclau and Mouffe (1985) do not deny that objects exist externally to thought but what they do deny is "that they could constitute themselves as objects outside any discursive condition of emergence" (Laclau and Mouffe, 1985, p.108). This has clear parallels with the philosophical position I have formulated in this chapter as a key underpinning for this inquiry, based on a merging of critical realism with constructionism. The principle of intentionality (Lyotard, 1991), which I employed as the basis for this critical realist interpretation of constructionism, where there is a "radical interdependence of subject and the world" (Crotty, 1998, p.45), can be seen to be at work here within Laclau and Mouffe's discourse theory. All objects are constituted in the interpretative and, in my view, intentional mode of discourse which means that everything in the social world is constructed discursively. Laclau and Mouffe assert that for the social world, "the linguistic and non-linguistic elements are not merely juxtaposed, but constitute a differential and structured system of positions - that is, a discourse" (Laclau and Mouffe, 1985, p.108). In other words, the entire system of phenomena in the social world, understood as existing upon the plane of intentionality, are constituted in discourse.

The position of discursive universalism held by Laclau and Mouffe (1985) is most consistent with the philosophical underpinning I laid out in section 3.3. Therefore, I adopted it as my position on the relationship between discourse and the social world. This position colours much of the analysis and theoretical generalisation which I perform in subsequent chapters, and the way in which I infer from points of analysis to make broader, more objective extrapolations about features of mental health services. I also view this to be a methodological clarification of my reconciliation of critical realism and constructionism since constructionist discourse analysis understood in this way is how the researcher ascertains more objective or real aspects 
of the world, constituted within a "discursive condition of emergence" (Laclau and Mouffe, 1985, p.108) or intentionality.

\subsubsection{Controversy surrounding theoretical generalisation in qualitative research}

The way in which theoretical generalisation is performed in this thesis in order to make broader, more objective claims about services could be seen as controversial given the restrictions which may be placed on qualitative findings. Most qualitative researchers express a commitment to viewing the social world through the eyes of the people they study (Bryman, 2016). Making general claims about the nature of services would seem to constitute knowledge which is beyond the scope of participants' perspectives. However, I do not believe that this potential limitation should be overemphasised. Arguments developed on the basis of cogency of theoretical reasoning have broad applicability because they characterise the ontological fabric of mental health services, structures and practice. In this sense, theory and analysis provide an 'ontological' snapshot of the processes occurring generally throughout services. I believe that this ontology is discoverable in the experiences of participants whose talk is analysed with rigorous qualitative methodology, which I have described in this chapter, and in robust development of theoretical argument on this basis. In this way, I would suggest that it is possible to step out of the confines of the idea of transferability in qualitative research to make bolder and broader claims, as I will endeavour to do in this thesis.

In many ways, the difficulty of qualitative generalisation can be seen as a version of the philosophical problem of solipsism, whose idealist philosophical position confines all possible knowledge to the subjective perceptions of an individual mind (Delanty, 2005; Guba and Lincoln 1994; Kenny, 2006; Rorty, 1980; see section 3.3.4). Solipsism can in turn can be seen as an extreme interpretation of the difficulties which emerge from the Cartesian dichotomy between subject and object. The Cartesian philosophical position faces the potentially intractable difficulty of extending knowledge from the subjective realm to the territory of extended and independent objectivity. However, in section 3.3.4, I embraced the principle of intentionality in place of this dichotomy, as a key underpinning for the epistemological position of this inquiry. Building on this, the principle of radical interdependence of subjectivity and objectivity (Crotty, 1998) was the basis for my approach to constructionism and my adopting 
the discursive universalism of Laclau and Mouffe (1985). It is through this epistemological position and understanding of discourse that I will connect more subjective features of talk with more objective attributes of services as I perform analysis and theoretical generalisation throughout the remainder of this thesis. The principle of intentionality is therefore the route to stepping out of the confines of the idea of transferability in my qualitative research. To confine the epistemological potential of qualitative research more tightly than this would, I suggest, fall under the critique levelled by Crotty (1998) towards much of qualitative research, which he accuses of being an idealist distortion of constructionism, and guilty of a "rampant subjectivism" (Crotty, 1998, p.48; see section 3.3.4).

\subsection{Chapter conclusion}

In this chapter, I have described the philosophical underpinnings of the methodology and its relationship to the specific methods employed according to the study design. After reiterating study aims and objectives, I clarified my interpretivist philosophical position in terms of methodology as that of constructionism combined contextually with critical realism. On this basis, thematic discourse analysis of qualitative semi-structured research interviews was deemed to be an appropriate method of inquiry for achieving the objectives of the research question. I followed this description of methodology with a discussion of research ethics issues. I then outlined my methods related to the manner in which I recruited participants in two phases and my sampling strategy. After this, I described my data analysis based on thematic discourse analysis which was also based on an interpretivist paradigm and contextualist constructionism. Finally, I discussed credibility and validity of my qualitative findings which are based on standards of quality and rigour supporting theoretical generalisation. I specified theoretical generalisation and discursive universalism as the bases for new knowledge to be generated by this study.

In the next three chapters, I will discuss the study findings produced by the methodology and analytical approach described in this chapter. In Chapter four, I employ complexity theory to analyse participants' talk generating the theme 'Misaligned expectations in negotiating transforming services'. I argue that service structure changes have led to the complex systems effect of misaligned expectations in negotiating the interface between primary and secondary 
care. This complexity is most manifest in the discursive practices, positionings and constructed meanings elucidated through my approach of thematic discourse analysis described in this chapter. This is one instance of my qualitative approach seeking to capture the experiential and perspectival dimension within participants' talk through thematic discourse analysis.

In a similar way in Chapter five, I generate through analysis the theme 'Competing versions of recovery' which identifies the way in which participants construct different meanings and approaches to recovery according to their own perspectival stances and agendas. In Chapter six, which is the third and final findings chapter, I focus my approach of thematic discourse analysis on experiences of care continuities and discontinuities which are constructed in participants' talk. This generates the theme 'Experienced discontinuities within primary mental healthcare'. 


\section{Chapter 4: Misaligned expectations in negotiating service structures and interfaces: a complex systems effect}

\subsection{Introduction}

In this chapter, I use the insights and sociological analysis provided by complex systems theory, introduced in Chapter two, to provide an analysis of my data and what is represented in the talk of participants. This process of interpretation led to the generation of the theme, 'Misaligned expectations in negotiating transforming services'. This theme refers to misaligned expectations participants have of transforming services, which impacts upon their ability to negotiate this interface, potentially engendering care discontinuities. The theme is relevant to the research question which focuses on care continuity and recovery for people at the interface of primary and secondary mental healthcare in Wales.

I have carried out thematic discourse analysis which constructs the theme 'Misaligned expectations in negotiating transforming services'. This was done in relation to two categories which are collated under this theme. These two categories are 'Discharge to primary care across the service system interface' and 'Referral to secondary care across the service system interface' which were developed through initial conceptual analysis. The theme refers primarily to a misalignment between expectations and changing service structures. The difficulty in negotiating primary-secondary interface boundaries, I argue, is a complex systems effect known as a 'wave of consequence' (Hannigan and Coffey, 2011). I argue that, according to analysis presented in this chapter, this phenomenon is most manifest in discursive practices, positionings and constructed meanings identified through discourse analysis, which builds on conceptual analysis to form the theme 'Misaligned expectations in negotiating transforming services'.

The theoretical context for qualitative analysis undertaken in this chapter was first introduced in section 2.5 on complex systems theory and mental health services. This theory is developed further in this chapter in relation to concept maps and data extracts of participants' talk on their experiences of care continuity and recovery. I concluded my review of relevant literature in section 2.5 by saying that mental health services in the UK represent an increasingly complex 
and fragmented system largely due to successive programmes of transformative, top-down legislation (Gilburt et al., 2014; Hannigan and Coffey, 2011). In Wales, the Mental Health (Wales) Measure 2010 can be seen as an instance of transformative top-down legislation in a devolved policy setting, which has introduced increased complexity into an already complex system. My investigation of care continuity and recovery experiences, for which the Measure is an important legislative context, can therefore be seen a candidate study of the kind of complexity described by Gilburt and associates (2014), and Hannigan and Coffey (2011) (see section 2.5 for preliminary discussion of these issues).

In the course of the following analysis, I will show how misaligned expectations of services are a key instance of a complex systems effect which may be revealed in participants' talk subjected to thematic discourse analysis. I argue that this complex systems effect or 'wave of consequence' is felt most keenly at the interpersonal level of social practice and interaction, or discourse. After introducing demographic characteristics of participants as my initial findings, I then present qualitative analysis of interview transcripts, which is illustrative of such complexity in Welsh mental health care and services, developing the theme of this chapter.

\subsection{Participant characteristics}

Study participants in Phase 1 consisted of people $(n=16)$ between the ages of 18 and 65 years, who had been discharged from secondary mental health services to primary care within the past three years. All these individuals had ongoing contact with third sector day centres in Wales. Participants were recruited in the context of their attendance of these third sector day centres. One exception to this was Ian who was recruited directly through poster advertisement for this research in a GP waiting room.

Phase 2 participants consisted of workers, professionals and clinicians $(n=16)$ also between the ages of 18 and 65 years, involved in supporting individuals discharged from secondary services in the past three years. These workers had direct experience of providing support for people who have transited between secondary and primary levels of Welsh healthcare services. They consisted of third sector organisation managers $(n=6)$, one third sector area manager $(n$ $=1$ ), senior and junior support workers $(n=6)$, and General Practitioners $(n=3)$. The total 
sample therefore for this study carried out in two phases was $n=32$ people using or providing services for those discharged from secondary mental health care.

In tables 4 and 5 in Appendix 7, I have summarised characteristics and demographic information for participants at both phases to provide a point of comparison with other studies and to help contextualise the accounts produced by individuals. Age-ranges have been provided since precise ages were not given by all participants. Classification of serious mental illness is entirely based upon the report of the participant.

Mental health problems reported by participants were bipolar disorder $(n=5)$, depression $(n$ $=6)$, anxiety $(n=7)$, post-traumatic stress disorder $(\mathrm{PTSD})(n=2)$, anorexia $(n=1)$, and intermittent psychosis related to epilepsy $(n=1)$. Some participants reported combinations of multiple mental health conditions. For both sets of participants, pseudonyms have been provided in order to protect their anonymity and affect a more personable approach for me to write about them in this thesis. Additionally, place and people names are omitted and substituted with generic labels to preserve anonymity e.g. $<<$ CMHT $>>$ in all data extracts used in the thesis. In the following section, I present the component parts of the theme of this chapter supported by data extracts and detailed analysis.

\subsection{Construction of the theme: "Misaligned expectations in negotiating transforming services"}

The theme presented in this findings chapter concerns systems complexity and mental health service boundaries at the interface of primary and secondary mental healthcare in Wales. As was described in section 2.5 , modern healthcare is becoming increasingly complex, and may be regarded as a complex system (Greenhalgh et al., 2010; Plsek and Greenhalgh, 2001). Particularly in modern mental healthcare, successive reforms and service transformations have led to a fragmented system and created a profusion of complex pathways which constitute contemporary services, which may be more difficult to navigate (Gilburt et al., 2014; Gilburt, 2015). One of the consequences of this is the existence of service boundaries at key points in the healthcare system, and the preliminary analysis of my data below shows that these are experienced by participants at the primary-secondary interface. 
Service users may move across the primary-secondary interface in two opposing directions, either they may be discharged from secondary services to primary care or they may seek referral back to secondary services from primary care. The theme 'Misaligned expectations in negotiating transforming services' sits across two categories derived from the data collected for this study, expressing this two-way care trajectory (Hannigan and Allen, 2013). These two categories are 'Discharge to primary care across the service system interface' and 'Referral to secondary care across the service system interface'. They are shown side by side in this chapter in Figures 5 and 6, since they are both directly related to the overarching category, 'Negotiating service systems interface boundaries', expressing movement in both directions across this interface. Categories are presented with various related concepts identified in the initial stage of conceptual analysis of my data. As can be seen from these figures, participants talked about experiences of care trajectories in both directions in terms of concepts relevant to both continuities and discontinuities of care. 


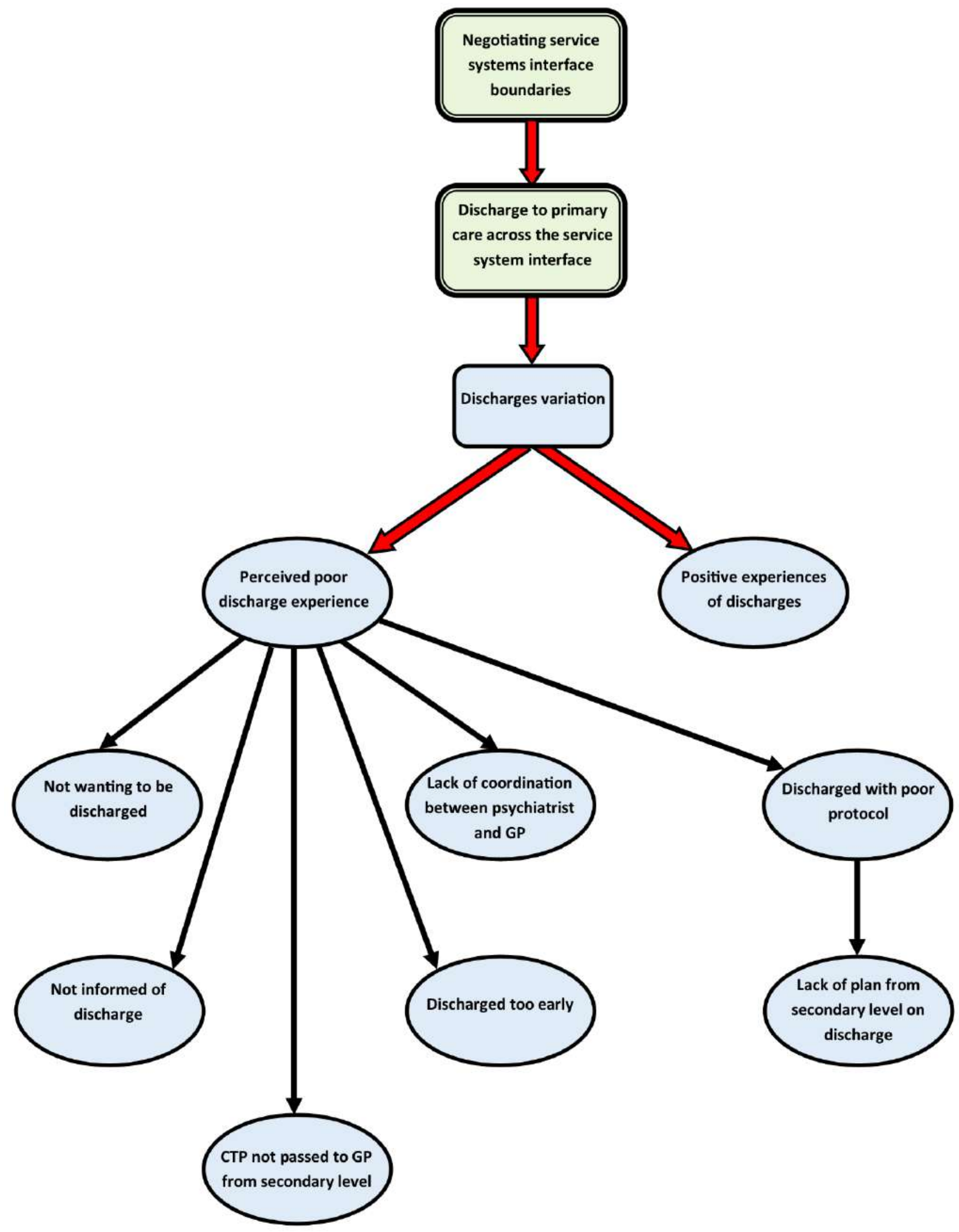




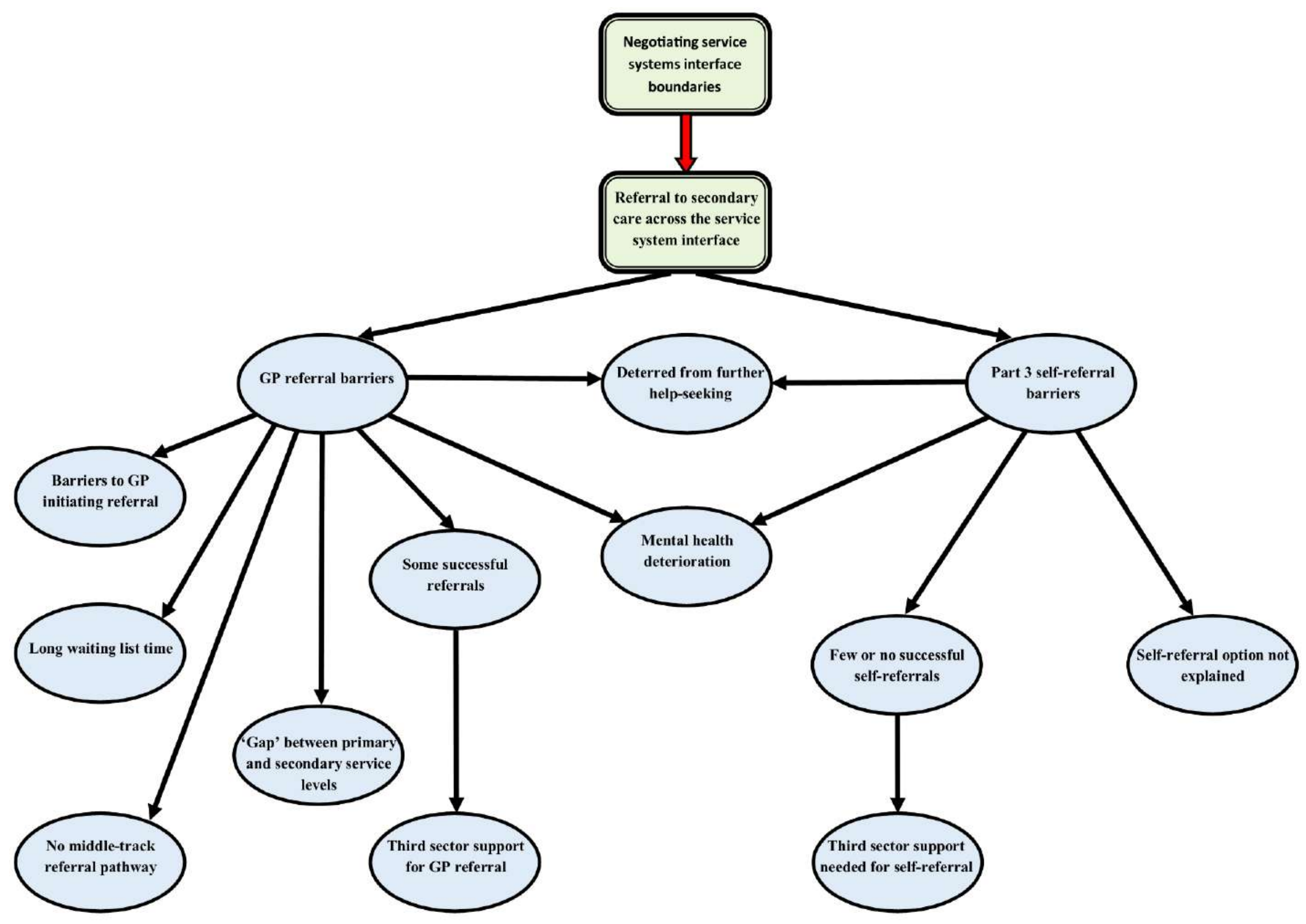


In the following sections, I first provide a summary of the concepts under the two categories represented in these figures, followed by discourse analysis of talk in data extracts related to concepts under these categories.

\subsubsection{Discharge to primary care across the service system interface}

The category 'Discharge to primary care across the service system interface' is related to the concept 'Discharges variation' representing variation in satisfaction with discharges experienced either directly or indirectly by patient or professional participants. Participants spoke about various difficulties experienced at discharge, represented by the concept 'Perceived poor discharge experience'. Participants also spoke about positive experiences of discharge, represented by the concept labelled 'Positive experiences of discharges'. However, variation in positive and negative experiences of discharge was weighted towards negative experiences. Only $n=6$ participants spoke about positive experiences of discharge from secondary care whereas $n=19$ participants spoke about negative experiences of discharge. In addition to this, $n=9$ participants spoke about the CTP not being passed from secondary services to the GP. This may be regarded as an instance of informational discontinuity (see section 2.7.7) since this is dependent upon the degree of communication between services and professionals and the level of consistency of care plans between different sectors (Weaver et al., 2017). It also runs contrary to the recommendation by the Welsh Government postlegislative assessment of the Mental Health (Wales) Measure 2010 that there should be "a clear interface and communication process between primary and secondary services... to ensure no service users fall through the 'gap"' (Welsh Government, 2014c, p.5).

One participant, James, described how he was discharged against his wishes (see section 4.3.1.2 where I perform discourse analysis of his talk). He was joined by a further four participants describing their views of what they constructed as the poor protocol with which their discharges were conducted (related to concept 'Perceived poor discharge experience'). Note that evaluations such as 'good' and 'poor' within concept map labels are the participants' constructed evaluations expressed through talk. In James' case, he described that he was still due to have specialist medical checks when he was discharged, which he could not access once he was in primary care. Other participants reported not having been sent a proper letter of 
discharge, a lack of preparation and discussion prior to discharge, and the lack of provision of a package of care. Two second phase participants, Maria and Tom, talked about instances where individuals under their care were discharged when they were still unwell and, in their opinion, not ready to be discharged (concept 'Discharged too early'). Finally, three participants, Sheryl, Lee and Alex, a third sector area manager, a junior support worker and a GP respectively, talked about a lack of coordination between the psychiatrist and the GP in the process of discharging a patient (concept 'Lack of coordination between psychiatrist and GP'). I commence discourse analysis of a data extract taken from the interview with the last of these participants, Alex, who is one of the three General Practitioners in my study. His talk covers a number of the care continuity issues identified in concepts under the category 'Discharge to primary care across the service system interface'. My analysis reveals professional and personal responses to transforming service structures engendered by the Mental Health (Wales) Measure 2010. It is at this experiential and intentional point that the complex systems effect of misaligned expectations of services in negotiating the primary-secondary interface is initially identified in this chapter.

\subsubsection{1 "There's not a plan for their future... you don't often see a strapline if things go wrong": Negotiating discharge plan documentation}

The following data extract is taken from the semi-structured research interview conducted with Alex, a male General Practitioner in his mid-thirties working and teaching in South Wales. Alex had previous professional experience within psychiatric secondary services whilst he was training as a medic. Whilst this experience has made him more open to practicing mental healthcare within primary care, he is keenly aware that not all GP's have had the same experience as him and may therefore not have the same level of expertise or interest in mental health. Alex's main criticism, made chiefly in the following data extract, is that there is a lack of clear guidance and information provided in discharge documentation from secondary care for specialist support and potential re-referral in the future. The culmination of my analysis shows that Alex constructs a complaint with regard to this experience. Edwards (2005) has argued from a discursive analytic perspective, that complaining can say as much about the subjective state of the speaker as it does about the thing being complained about. I will show how this this understanding of discourse as a complaint reveals issues about Alex's 
expectations and perspectives of service behaviour, which are of greater analytical interest than perceived service system boundaries or care discontinuities his talk refers to.

The following extract is a response to an opening question about General Practitioner provision of care and treatment to people with SMI who have been discharged from secondary services:

1. $\quad$ Researcher: If I could just start with a sort of general

2. question, what is your experience of providing care and

3. treatment to people who have been discharged from

4. secondary services that have a serious as opposed to

5. common mental health disorder?

6. Alex: Erm (.8) mixed I suppose. I've worked in a few

7. different surgeries over the years because I used to do

8. some locum work so I'd see different people being

9. discharged from different facilities and from different mental

10. health (.) community mental health teams. So sometimes, as

11. with any other secondary to primary care transition,

12. sometimes that's quite smooth and well covered and

13. there's good support and good discharge documents and a

14. good plan for the future and sometimes it's hopeless. And

15. it's a very movable thing, it just depends on the person

16. you're dealing with - teams you're dealing with really. Erm, I

17. think overall, the documentation is (.8) - that you get back

18. is often very detailed and lengthy which is good but it's

19. quite hard going to get through it sometimes. Erm, but I

20. don't know if there are very good plans going forward, so

21. people are often discharged on ' $x$ ' medication, but there's

22. not a plan for their future, there's not (.6) you don't often

23. see a strapline if things go wrong - if things go badly

24. wrong, if things are struggling just, just (.) you can contact us

25. on this number or we can get them seen very easily or this

26. is what we want done with the medication if this happens

27. so there's less of a long-term plan. And of course then as a

28. GP when those eventualities occur, you don't have many

29. options other than picking up the phone which doesn't

30. always reap rewards 'cause you can't get through to

31. somebody or you have to refer back through the whole

32. system and that can be quite lengthy and bounce back and

33. all that sort of stuff, so that's a frustration that's not

34. uncommon.

35. Researcher: Okay, so I think you were particularly

36. focusing on a kind of plan for a crisis situation when things

37. kind of deteriorate and having some sort of clear, laid out

38. plan for the best course of action, potentially getting them 
39. back into specialist services. Is that the kind of thing that is

40. lacking?

41. Alex: Yeah, not just in the event of a crisis, sometimes the

42. opposite. So if the patient seems to be doing very well with

43. whichever antipsychotic they might be on, for example, at

44. what point do we reduce this and at what point do - should

45. we consider increasing it again if things are drifting off? At

46. what point do we get secondary care, psychiatric services

47. involved again? and these things are often missing from the

48. otherwise very detailed documents.

[Alex, General Practitioner, Phase 2, Participant 15, lines 1-48]

Alex responds to my initial question about GP care provision by shifting the topic immediately onto an account about variation in perceived quality of discharges from secondary care. Discharge is "a very movable thing" (line 15), it "depends on the person you're dealing with" (lines 15 to 16), or the "teams you're dealing with" (line 16). It is noticeable that the main topic of my question is not however about variation in perceived quality of discharges but about primary mental healthcare. That Alex focuses on a topic in his talk different to that posed in my question is indicative of his intentional focus on the topic of difficulties he perceives with secondary service discharges. James Paul Gee (2010) argues that the way in which a speaker organises and negotiates topic flow and topic shifts is a strategy for building the particular view of the world they are constructing. In this case, Alex's topic shift is indicative of his concern to develop and promote his complaint about the problems he perceives with discharges, rather than an examination of primary mental healthcare itself. This complaint then leads onto an account of perceived lack of adequate specialist support, a lack of a long-term care plan within discharge documentation, and perceived difficulties in accessing secondary services through referral.

Throughout this data extract, Alex develops his topical focus on the limitations of discharge documentation and specialist support. Whilst documentation is "often very detailed and lengthy which is good... it's quite hard going to get through it sometimes" (lines 18 to 19). Furthermore, according to Alex, there are key omissions in the lengthy documentation provided. What is lacking according to Alex is what he refers to as a "long-term plan" (line 27) which would give a clear indication of what to do if medication adjustment should be appropriate or if the patient was doing well over a period of time. This issue is then developed 
into a complaint about a lack of collaborative support provided by specialist, secondary care (lines 41 to 48). In Alex's view, this might be preferable to the option of simply referring the person back to secondary care (lines 28 to 34 ). Alex legitimises his complaint by constructing his identity (Antaki and Widdicombe, 2008) as someone with enough varied experience of mental healthcare to legitimate his complaint. He does this by referring to his experience of working in GP surgeries in the past where he has seen numerous different types of potentially problematic discharges from the CMHT (lines 6 to 10). This legitimation is further added to by the context of talk in the wider interview where he refers to his previous professional experience within psychiatric secondary services whilst training as a medic.

The abrupt manner in which the word "hopeless" (line 14) is used to end initial talk about good discharges (lines 10 to 14) also serves to emphasise this negative account. Additionally, Alex's talk about good discharges is tentative, due to being qualified by the adverb "sometimes" (line 10), so that he does not emphasise the discharges that have gone well but rather the ones he views as having gone poorly. Finally, the emotion category report of frustration in line 33 serves to amplify the effect and credibility of his critique, which is one of the discursive functions of this kind of rhetoric (Wiggins, 2017). Emotional categories are instances where the speaker makes relevant a particular category of emotion or report of an emotional state in their talk, in order to achieve a particular discursive function and emphasis (Wiggins, 2017), which in this case is to amplify Alex's complaint. By the use of these various discursive devices therefore, Alex constructs his negative account or complaint with regards to discharge documentation and secondary services.

Alex then goes on to describe his aversion to lengthy and difficult discharge documentation, introduced at line 18. I viewed this discursively as a case of Alex positioning himself and other GPs as the prime recipients of overwork in comparison to other clinicians and professionals in the NHS. All three of the GP participants follow this vein in speaking about having a very heavy workload, which they argue is the result of increased healthcare demand from the population accompanied by resource limitations placed upon services. Other participants $(n=$ 11) talked about their perception of GPs being stretched to capacity or being too busy to provide often complicated mental healthcare (see concept 'Limited service capacity in relation to demand' in Figure 12). The notion that GPs are already too busy to engage in the additional 
task of treating complex mental health cases does find support in the literature. Some studies have identified that one of the major limitations for GPs to carry out effective mental healthcare is excessive workload pressures due to high caseloads (Gofal, 2013; Wilkinson, 2015). Consequently, there is a reluctance to take on new patients with SMI who often have complex needs (Gask et al., 2003; Gofal, 2013; WaMH in PC, 2015). However, as was outlined in section 1.2, increased levels of primary mental healthcare is a consequence of the Mental Health (Wales) Measure 2010. The reluctance which Alex exhibits with regard to taking on these increased levels of complex care, framed within a context of reported difficulties with discharges and documentation, can be seen as an example of the kind of complex systems effect which was discussed in section 2.5.2. Here, I outlined that a constantly changing and increasingly fragmented system, impacted by top-down transformative programmes such as the Measure, have led to the complex systems effect of shifted roles and responsibilities, and resultant tensions between professional groups (Gilburt et al., 2014; Hannigan and Coffey, 2011; Pressman and Wildavsky, 1973). The difficulty Alex has with secondary service discharges and documentation is an expression in his talk and the data set of these kinds of increased tensions between professional groups within primary and secondary sectors due to new roles being adopted.

Further supporting this analysis, it is notable that the various discursive devices described above are employed by Alex to create an account which directs the blame entirely away from himself or from the primary care sector. His account is constructed to achieve a total absolution of GPs for their part in shortfalls in service provision. Therefore, the above extract and surrounding interview may be understood as a type of account known as an excuse (Orbuch, 1997; Scott and Lyman, 1968). Scott and Lyman (1968) identify two types of accounts which may occur in talk: justifications and excuses:

"Justifications are accounts in which one accepts responsibility for the act in question, but denies the pejorative quality associated with it. Thus, a soldier in combat may admit that he has killed other men but deny that he did an immoral act since those he killed were members of an enemy group and hence 'deserved' their fate. Excuses are accounts in which one admits that the act in question is bad, wrong, or inappropriate but denies full responsibility"

(Scott and Lyman, 1968, p.47) 
Full responsibility, or indeed any responsibility at all, for difficulties in delivering successful primary mental health care is omitted from Alex's account which may be considered therefore as an example of an excuse, in the sense provided by Scott and Lyman (1968). This excuse is constructed to shift the blame elsewhere: onto secondary services who are responsible for problematic composition of discharge documentation, and onto an environment of excessive workload pressures being imposed upon GPs from external sources.

However, the picture in contemporary mental health services can be considered to be more complex than the particular view which Alex constructs in his discourse. Although Alex's focus is on the workload pressures of GPs, it can be pointed out that these are distributed fairly evenly across the entire workforce. Whilst it is true that GPs currently struggle with a high level of occupational stress (WaMH in PC, 2015; Wilkinson, 2015), this is also true for workers in secondary care (RCPsych, 2014), including CPNs and ward mental health nurses (McKeown et al., 2017; Simpson, 2005). In the light of these considerations, Alex's proposed solution that a "succinct document" (line 389) be provided at the point of discharge can be better understood as a product of his positioned account, than as a constructive and realistic suggestion. Requiring secondary services to do the extra work of providing a succinct document would merely shift the problem of dealing with complex mental health cases in the context of excessive workloads elsewhere within services, and in this sense is not a solution to the problem at all. Summarising the complex case notes of persons with SMI in such a succinct document without omitting important details would not be an easy task, and potentially an onerous one considering the workload pressures CMHTs are already under.

A more balanced approach to solving the kinds of problems Alex is experiencing might involve implementing increased GP mental health training, which is lacking in the UK and is not a mandatory requirement (Currid et al., 2012; Fleury and Grenier, 2012; Freeman et al., 2002; Gofal, 2014). This could be done at the same time as improving the levels of informational continuity within discharge documentation, which is Alex's main focus. Alex's talk is positioned in such a way that it does not take this balanced view. Rather, it places the problems facing mental healthcare and their potential resolution firmly at the doorstep of secondary care. 
A phrase which Alex uses which is of analytical interest is "long-term plan" (line 27). This term is not precisely defined in Alex's talk and may therefore be better understood non-literally (Wood and Kroger, 2000) as a 'figure of speech' or 'form of speech'9 referring to an unspecified care pathway which is more sophisticated than just the one-dimensional route of often slow referral back to secondary services, should a patient's condition deteriorate (see lines 27 to 34). Alex elaborates in non-specific terms about what this long-term plan might entail. It would be an approach which would involve more than simply referring someone back to secondary services if they became clearly unwell, since there can be many difficulties associated with this approach (lines 27 to 34). A "long-term plan" (line 27) might also be an alternative to referral altogether, where there is greater collaborative support from specialist services (lines 24 to 25 and lines 45 to 47). Alex also talks about the long-term plan involving receiving guidance from the secondary sector if the patient was doing well and medication reduction seemed appropriate (lines 41 to 47). His talk also accords with Glasby and Tew's (2015) point that:

"Not all mental health work fits neatly within a neat 'assessment-treatment-discharge' model which underpins much of general healthcare, as many people having more long-term, fluctuating and complex needs... do get better but not in a very straightforward or linear fashion."

(Glasby and Tew's, 2015, p.22)

In line with this thinking, Alex's proposal is for a different approach where patients with complex needs are treated in the context of a less linear systems interface between primary and secondary care. Rather than being inflexible and unresponsive, mental health services should have clear point of access and flexibility to account for variations in presentation (Gilburt et al., 2014). A flexible system would allow better opportunities for specialist mental health professionals and facilities to provide support for primary care in areas such as medication management and long-term support in cases of patient improvement (WaMH in PC, 2015; WHO, 2008). Such a system of collaboration could provide an alternative to simply referring the patient to another part of services when there is a change in healthcare needs.

\footnotetext{
${ }^{9} \mathrm{~A}$ form or figure of speech may be defined as "a word or phrase used in a non-literal sense for rhetorical or vivid effect" (Oxford Dictionary, https://www.lexico.com/en/english).
} 
In support of Alex, the service system as it stands in the UK and Wales could be considered to be simplistic and one-dimensional in that that there exists only a fast-track and a slow-track care pathway for individuals to be referred back to secondary services (Welsh Government, 2014a). The slow-track pathway is the standard referral pathway whereas patients can be referred more quickly in cases of emergency either through the GP or the crisis resolution and home treatment team (CRHT). However, a variety of different possible models have been proposed for collaboration between primary and specialist mental health services, including shifted outpatient clinics, attached mental health nurses and a consultation-liaison model (Durbin et al., 2016; Freeman et al., 2002; Gask et al., 1997; Lester et al., 2004). In many ways, Alex seems to be describing something similar to the latter of these, the consultation-liaison model for integrated primary mental healthcare described by Lester and colleagues (2004), where "primary care teams are provided with advice and skills from specialist mental health services" (p.287).

However, most of these innovations would come with costs and may not be easy to implement in Welsh services. Alex's positioned account omits that there could be ways to deliver better mental healthcare for people with SMI within primary care itself. GPs negotiated a contract bonus under the last Labour government where they undertook to do various forms of additional mental health care (BMA, 2003; Charlton, 2005). For example, a new role of General Practitioner with Special Interests (GPwSIs) was established in this contract, which includes a potential specialisation in mental health (BMA, 2003; BMA, 2018). Recent updates to the contract in Wales have also included clear directives towards enhanced primary mental healthcare services (BMA, 2018). One could argue that these contracted roles could and should fit neatly with the purposes of the Mental Health (Wales) Measure 2010 in seeking to implement greater levels of primary mental healthcare.

Despite these contractual agreements, Alex and the other two GP participants, Robert and Tamsen, both admit limitations in mental health expertise within primary care and a lack of training, since it is not a mandatory requirement. This accords with the literature indicating that there are limitations on the amount of training and mental health expertise amongst GPs (Durbin et al., 2016; England et al., 2017; Fleury and Grenier, 2012). One glaring example of this lack of expertise in the data set is the observation that none of the three GP participants 
have any awareness of the concept of 'recovery', which has become a dominant rhetoric and paradigm within mental health care and services (Braslow, 2013; Deegan, 1988; Edgley et al., 2012; Gilburt, 2013; Repper and Perkins, 2003). Primary care has frequently been criticised for delivering a poor standard of mental healthcare delivery (Docherty, 1997; Ramanuj et al., 2015; Tiemans 1996), and the issues raised here would seem to confirm this verdict. Given the presence of these problems, and their apparently not having been properly addressed, it might be said that a more realistic contract negotiation in 2003 and 2018 would have been for GPs to reject an enhancement of primary mental healthcare delivery altogether.

In sum, Alex presents in his talk a variety of ideas and expectations about services including better discharges, more succinct discharge documentation (line 389), and a "long-term plan" (line 27) involving more collaboration with and support from specialist services, and a less linear system of referral at the primary-secondary interface. Whilst this might seem a reasonable set of innovations, discourse analysis of his talk points towards issues concerning a lack of ownership of responsibilities for shortfalls in primary mental healthcare, despite successful contract negotiations for enhanced primary mental health delivery. I argue that analysis leads to an interpretation of Alex's talk as a highly positioned account, rather than to a preoccupation with appraisal of the value or feasibility of proposals he outlines. This positionality is indicative of tensions between professional groups which have resulted from shifted roles and responsibilities in an increasingly complex and fragmented system. Alex's expectations of services are therefore representative of his own misalignment with changes in primary mental healthcare delivery, in the context of shifting service structures and new professional roles and responsibilities (Hannigan and Coffey, 2011). This may itself be considered to be an instance of complexity in the light of healthcare theory presented in section 2.5 (Gilburt et al., 2014; Hannigan, 2013; Plsek and Greenhalgh, 2001), which is inherent in the difficulties Alex has in negotiating primary-secondary interface boundaries in delivering primary mental healthcare. In conclusion, Alex's talk can be seen primarily as a complex response to a complex scenario.

Concluding this stage of analysis, it can be observed how adding an interpretivist dimension of qualitative analysis through employment of discourse analysis builds greatly upon conceptual analysis to fashion the in-depth qualitative approach described in section 3.6. Although the 
concept map in Figure 5 appears to indicate that there are a variety of objective problems with discharges, closer examination of one participant's talk reveals that the main issues of negotiating service interface boundaries lie in the perceptions and expectations of this individual. In this way, discourse analysis, building on top of conceptual analysis, allows me to grasp analytically the interpretive dimension in participants' talk which is precisely where the complex systems effect of misaligned expectations lies.

The argument I have initiated here in support of the theme 'Misaligned expectations in negotiating transforming services' will be developed further by discourse analysis of the talk of other participants. In the next section, I continue to analyse talk which has been coded under the category, 'Discharge to primary care across the service system interface'. This involves analysis of James' talk. Whilst Alex raised a number of difficulties with the discharge process, the next participant, James, talks about actually being discharged at all being problematic for him. I shall argue that analysis of James' talk further illustrates the complex systems effect of misaligned expectations in the context of shifting service structures.

\subsubsection{2 "All I got told was they were going to discharge me": Being discharged against one's wishes}

James is one participant who is explicit in his interview that he did not actually want to be discharged from secondary services. James is a man, in his mid-fifties living in a rural area of West Wales, who has used both primary and secondary level services. He suffers from a mental health problem linked to organic brain damage. Up until recently, he had received his care entirely through a CMHT in another area. James then moved home and his care was then provided by a new CMHT. This transfer of care was the beginning of his difficulties, as he expresses them, since he was discharged to the GP after a period of less than a year within this new service context. Neither he nor his family wanted this discharge to occur, as the following data extract illustrates:

73. James: Yes, yes, so the community mental

74. health team in $<<$ area of Wales $>>$ when I left there.

75. Researcher: Yes, yes, so you weren't happy with

76. their care, and then they discharged you to the GP? 
77. James: Then, they discharged me to the

78. GP, yes.

79. Researcher: So, did you wish to be discharged

80. to the GP?

81. James: No, I didn't wish to be discharged,

82. no.

83. Researcher: So, how much..?

84. James: Neither did my family.

85. Researcher: Yes, how much say did you have in

86. the decision to discharge you to the GP?

87. James: Ultimately, nothing. I was just told,

88. you know, there was nothing...(.5) What more did I expect

89. them to do, and what could they do for me, you know? I

90. was having talking therapy on a regular basis with my

91. previous mental health team. I had regular contact with a

92. worker, with a mental health nurse, and when I moved

93. down here, I was allocated a mental health nurse who I

94. saw, what, three times, I think it was.

95. Researcher: In the space of how long?

96. James: In the space of, what...(.4) Well, I

97. moved down here in January 2015, I got taken over by this

98. team here in, I think it was the end of March 2015. I saw

99. <<--->> for about, I think, three times, from March

100. till I think it was the August, when she left. I didn't see

101. anybody then, before I got allocated another worker, for

102. about two months. Then, when I got allocated another

103. worker, all I got told was they were going to discharge me.

104. Researcher: Right, okay, so the third worker=

105. James: =That was the third worker, yes.

106. Researcher: Did you ever meet the third worker?

107. James: I did meet her, yes, yes. I just didn't

108. like that she was very...(.3) I just didn't like...(.2) Everything

109. seemed to be in a rush, she was all hurrying about, she

110. was in a rush all the time. She'd come in, she'd talk to you,

111. and she didn't know anything about my history or anything,

112. because she'd never read my notes, right? She admitted

113. that, she'd never read my notes.

114. Researcher: But, they were available to her?

115. James: Obviously, they'd been transferred,

116. and my wife was with me at the time of the interview, so my

117. wife had to tell her what was in my notes, and she said,

118. "Well, I'm not aware of that, because I haven't seen that",

119. she said. That was why I was still under the care of a

120. mental health nurse in $\langle<\mathrm{CMHT}>>$, you know. I was seeing

121. them regularly. Anyway, that seemed to have been

122. forgotten again by this mental health nurse.

[James, Service user, Phase 1, Participant 8, lines 73-122] 
The support of his family in line 84 can be seen as an intersubjective corroboration (Baruch, 1981; Jingree and Finlay, 2013) of James' claim that it was not right for him to be discharged. In addition to James not wanting to be discharged but being discharged anyway, it appears from his talk that he was not given the opportunity to provide any input into this decision (lines 87 to 88 ). The identity of the discharging nurse is constructed in a critical fashion via a negative portrayal of her being "in a rush all the time" (line 110). This portrayal of care is meant to be negative as it is something which James twice says he "just didn't like" (lines 107 and 108). This is enhanced by intersubjective corroboration from his wife (lines 116 to 117) and the report that the nurse did not know anything about his history or notes (line 111 to 118).

In addition, James details a scenario in his talk where he is provided with a number of workers by the new CMHT in contrast to the regular contact with one mental health nurse he had under the previous CMHT (lines 91 to 103). The arrival of the final worker is accompanied by him being informed that he is going to be discharged (line 103). The combination of rhetorical devices, the use of contrast, repetition of the phrase "another worker" (lines 101 and 102), and the topic flow from a picture of satisfactory care to piecemeal care ending with discharge, has the effect of constructing a negative depiction of his treatment by services. Such a construction resonates with literature on longitudinal and relational (or therapeutic) discontinuities of care (Biringer et al., 2017; Burns et al., 2009; Sweeney et al., 2016; Ware et al., 2003). Longitudinal continuity is having care delivered by as few professionals as possible with minimal gaps in treatment (Burns et al., 2009; Ware et al., 2003). Relational continuity concerns the degree and quality of therapeutic relationship which is formed between the patient and the clinician (Burns et al., 2009; Freeman et al., 2002). There is potentially a connection between these two types of care continuity as is illustrated by James' talk. He describes being allocated to a series of workers for short periods of time with no statement about the establishment of a new therapeutic relationship involving "regular contact" under the new CMHT (line 91). This decline in the level of secondary care culminates in complete discharge (line 103). I read this as a form of topic-chaining (Gee, 2010) wherein a chain of linked events is constructed to create a coherent sense of the gradual decline in secondary care provision, from James' point of view. This can be seen as a process of textual meaning-making through 'texturing' equivalence and difference between phrases in talk, in this case expressing a gradual reduction in care-level 
over time (Laclau and Mouffe, 1985). The difference is between the previous situation of "regular contact" (line 91) with easy and sustained access to secondary services on the one hand, and, on the other hand, the current state of affairs involving no contact with services due to discharge. Equivalence is achieved by combining different instances of talk about increasingly discontinuous care so that they create a sense of a coherent process of his care provision being reduced. The point is that it is not necessarily the case that this coherent process of care reduction in lines 91 to 103 is objective and therefore an explanation ('Erklaren') for the ultimate removal of specialist care. Rather this is a particular interpretation or 'Verstehen' (Manicas, 2006) which James promotes in his talk. James constructs his talk in such a way that it appears to provide an objective explanation of the cessation of care at the secondary level. Yet the discursive functioning of his talk indicates that this may be his own overlay constructed in the meaning-making process of his interview and discourse (Holstein and Gubrium, 2004).

An alternative explanation for the discontinuities of care James talks about may be found in a complex-systems understanding of the impact of the Mental Health (Wales) Measure 2010, as opposed to a real agenda amongst clinicians to side-line him, which what his discourse in this extract suggests (lines 103 and 122). Part 2 of the Measure specifies a mandatory requirement that all people who are treated in secondary services have a Care and Treatment Plan (CTP) and a care-coordinator (Hannigan et al., 2018; Welsh Government, 2010b; 2011a). These two new requirements have led to the process described in section 1.2 whereby a greater than expected number of people with SMI have been discharged to primary care. In section 2.5.2, I described this as a complex systems effect referred to as a 'wave of consequence' (Hannigan, 2013; Rittel and Webber, 1973) resulting from implementation of the Measure (Gofal, 2015; ORS, 2014; Welsh Government, 2014c).

It appears that James has had direct experience of this phenomenon. The problem of high caseloads and busy diaries held by CMHTs (Hannigan, 2013) is supported in the above extract where James speaks about his care coordinator seeming to be in a "rush all the time" (line 110), and the high turnover of care coordinators (lines 92 to 103). This was the initial problem in secondary care meant to be resolved by the effect of the Measure in moving patients to primary care (Welsh Government, 2013; 2016). In so doing, CMHTs could be freed up to focus on providing care for those with the greatest need for the right amount of time. In this way, it is 
intended that prudent healthcare principles would be achieved, focusing resources on individuals with the greatest need (Aylward et al., 2013). At the same time, it was intended that the Measure would promote recovery and self-management approaches for those who have been discharged to primary care (Davidson, 2005; Welsh Government, 2016).

As a consequence of this, services in Wales have become more orientated to primary care and accompanying self-management recovery approaches. This has involved increased levels of discharges, which is something James has experienced in a direct way. But, as with Alex, the key issues is that James' expectations are arguably not properly aligned with this new services behaviour. I argue that a further 'wave of consequence' emanating from the implementation of the Mental Health (Wales) Measure 2010 is a mismatch between service users and worker expectations and the new state and behaviour of services under the Measure. Rittel and Webber's (1973) concept of a 'wicked problem' is an appropriate concept with which to understand this phenomenon. The concept of 'wicked problems' provides a framework within which it may be understood how unpredictable and detrimental results are produced by policies intended to do good, in a complex healthcare system. In this case, the wicked problem is inherent in the complexity of mental health services and the need for these to be increasingly recovery-oriented. The Mental Health (Wales) Measure 2010 is the solution to this wicked problem. However, according to complex systems theory, solutions to wicked problems generate 'waves of consequences' which are unpredictable, unintended and even counterproductive (Hannigan and Coffey, 2011). I outlined in section 2.5.2, that a greater than intended level of discharges is a 'wave of consequence'. A further 'wave of consequence' evidenced in my data analysis is the discrepancy between the expectations of people like James and Alex and the new behaviour of services.

As with Alex, the greatest level of complexity lies at the point of James' subjective positioning in relation to changing service structures, revealed in his talk, which shows the inconsistency between his views and the implications of health policy. It appears that people are finding the boundary at secondary care impermeable and inaccessible from the primary care side resulting in experiences of bouncing off the service interface and feeling that they are not getting a fair hearing for their concerns about their mental health. For James this inaccessibility of secondary services leads to his experience of being rejected by secondary services against his wishes. In 
my conceptual analysis, ten participants specifically spoke about their difficulties and frustrations in accessing secondary services, mostly in the discursive form of complaints (See concept 'Part 3 self-referral barriers' in Figure 6). This included being turned away by CMHT receptionists and told to go back to their GP (according to James' account, see data extract in section 4.3.2.2 below). All of these effects could be considered to be the same wave of consequence or complex systems effect of misaligned expectations about transforming services.

Later on in the interview, James goes on to construct what in his view is the real agenda to discharge numbers people in order to reduce the size of CMHT caseloads:

133. James: That was her agenda from start to

134. finish, was just to discharge me. At that time, there were

135. quite a few other people, in this - that attend this building. They were all

136. being discharged.

137. Researcher: Oh, other people were being

138. discharged?

139. James: Yes.

140. Researcher: Going through the same process?

141. James: Yes, they were all going through the

142. same process.

143. Researcher: Yes, yes.

144. James: So, I realised then, I wasn't alone,

145. you know? I just thought it was something to do with these

146. cut backs, austerity, saving money, and getting people

147. off their books.

[James, Service user, Phase 1, Participant 8, lines 133-147]

This intersubjective corroboration of his experience in the reports of other patients serves to enhance his construction that there is a real problem with people being rebuffed from secondary services. At the end of this extract, James introduces his political perspective into his discourse by the use of the terms "cut backs" and "austerity" (line 146) which he directly relates to "getting people off their books" (line 146). These discursive terms are what Gee $(2004 ; 2010)$ refers to as political discourse. Gee views the handling of discursive terms from the political sphere as a way in which speakers provide an ideological context for their talk. According to critical discursive analysis, speakers may actively employ a particular position to support one side of an ideological dilemma (Billig et al., 1988). Ideological dilemmas are often expressed 
rhetorically through the use of interpretative repertoires (Potter, 2003). In James' talk, an interpretive repertoire drawing on the concepts of "austerity" and "cut backs" (line 146) is used as a coherent way of talking about his perspective on the increased phenomenon of discharges from secondary care. Interpretive repertoires are a central feature of discursive psychology defined as "systematically related sets of terms that are often used with stylistic and grammatical coherence around one or more central metaphors" (Potter, 1996, p.9). Various rhetorical devices and techniques are employed by James for the purposes of producing the interpretive repertoire supporting his particular interpretation of the situation being talked about (Davies and Harré, 1990). James provides a context and explanation for discharges by invoking topical cultural notions of austerity-based politics. The alternative view on the other side of this ideological dilemma is the opinion that it was a legitimate policy intention of the Mental Health (Wales) Measure 2010 to free up CMHTs which were already heavily burdened (ORS, 2014; Welsh Government, 2016). However, James' talk is discursively constructed to promote a complaint bolstered by the suggestion that the service restructuring he is facing lacks legitimacy.

One participant, Tom, who is a senior support worker in a third sector day centre, explicitly describes this issue of there being a discrepancy between some critical patient viewpoints about services and service realities. In the following data extract, he describes how the influence of such constructed and subjective viewpoints makes it difficult for him to objectively evaluate the appropriateness of patient discharges:

42. Researcher: Do you feel that all the people who are

43. discharged from services to their GP, do you feel that all

44. of those people are discharged in the right way, when

45. they're ready to be discharged?

46. Tom: No, not all of them. But it would

47. be hard to put a number on it, because, speaking to

48. people here, we only have their side, or their opinion, of

49. it. So some people may forever be of the opinion that

50. they should be under secondary care. Whereas they are

51. actually ready to...(.2) And maybe it's an anxiety or a fear,

52. or a need that they want to stay with what they are. But

53. also there are people that aren't ready, in the same

54. things. So sometimes it's hard to judge whether they are.

55. Because we are only going on what they've told us. But 
56. on occasion, personally, you do think, "Oh, this person

57. doesn't really seem well enough, to be going in the

58. direction that they're going."

59. Researcher: So there are definitely some

60. cases?

61. Tom: Some cases. $=$

62. Researcher: $=$ Yes. $=$

63. Tom: =Where, I think if you spoke to

64. service-users, you would have an inflated number of it.

65. Because there will be the ones that they feel they are not

66. ready, when really they are.

[Tom, Senior support worker, Phase 2, Participant 8, lines 42-66]

Despite Tom saying that there are "some cases" (line 61) of individuals who are discharged when they are not ready for primary care, his main point in this data extract is that there are many services users who do not wish to be discharged even though this may be a suitable care trajectory for them (lines 65 to 66). The issue is that "some people may forever be of the opinion that they should be under secondary care" (lines 49 to 50), which, in terms of an emotional category or report, may be 'anxiety or fear' driven (line 51) and therefore not so objectively valid. Consequently, Tom finds it difficult to respond to my question about the appropriateness of discharges. Since he is only recipient of information provided to him by clients within his organisation who might have a misaligned view of service provision, his answer would entail an "inflated number" (line 64) of participants who would apparently be inappropriate discharges. This data extract from Tom's interview seen in the light of James' account shows that there are competing viewpoints about negotiating transforming services and interfaces, and what is classed as appropriate or inappropriate service provision. This diversity of views and expectations held by James, Tom and the other service users referred to in their talk contributes to my argument here about escalating service complexity. This is manifested most keenly within the subjective positioning and discursive practices of participants in their talk concerning often misaligned views about transforming behaviour of services.

\subsubsection{Referral to secondary care across the service system interface}

I now move on to discuss concepts under the category 'Referral to secondary care across the service system interface' and related talk. This category has a number of sub-concepts which are shown in Figure 6. Boundaries to referral to secondary care were discussed by $(n=15)$ 
participants in a total of 78 coded points in the data, making it one of the most prevalent concepts described in the data. The category 'Referral to secondary care across the service system interface' is the parent of two sub-categories, 'GP referral barriers' and 'Part 3 selfreferral barriers'. Respectively these sub-categories stand for prevalent patterns of content in talk about barriers to successful or timely referral to secondary services by the GP, and barriers to successful or timely self-referral to secondary services under Part 3 of the Mental Health (Wales) Measure 2010. I now provide further discourse analysis to enhance the conceptual analysis provided in Figure 6. Data extracts of talk are taken from the research interviews of two participants, Alun and Katy. Alun talks about his difficulties in accessing secondary services through GP referral and Katy about similar difficulties through self-referral.

\subsubsection{1 "My biggest problem is that I don't have a CPN": Perception of secondary service inaccessibility}

Alun devotes a considerable amount of his interview to a complaint about perceived inaccessibility of secondary, specialist services through GP referral. Alun is a male in his midfifties suffering from bipolar disorder, a condition he has had for many years. An acute relapse of his mental health condition is a future scenario which he is concerned about, a worry which he connects directly with the difficulties he describes in accessing specialist mental healthcare support provided through a community psychiatric nurse (CPN). This state of concern is exacerbated by a traumatic experience he had in the past on discharge from hospital where he attempted suicide by jumping off a bridge.

The data extract presented here occurs early on in the research interview, following an initial discussion about a previous hospital admission. This treatment and care is described as a relatively uneventful experience after which Alun expresses a positive evaluation of hospital services and the GPs under whose care he was placed upon discharge. However, this discussion turns out to be a primer for his complaint about a lack of access to specialist, secondary care which he asserts would provide the level of care quality he needs, similar to that received previously when he was in hospital: 
56. Alun: Yeah, subsequently within

57. several days of coming out of hospital I was feeling a

58. darn sight better. So that was good. That treatment was

59. good, it did the trick, it was short term. I didn't want to

60. spend a long time in hospital.. uhm.. (.2) I was visited by

61. the crisis care team when I came out, and so then once,

62. and then it was back to the GP. With the nature of my

63. illness it fluctuates, and erm, a couple of weeks down the

64. line I was feeling unwell again. And then I was

65. with the doctor and then my doctors are great: they know

66. me, they know my condition and they know uhm - I'm

67. able to talk to them clearly and explain how I'm feeling,

68. uhm succinctly and uhm I do get the care I need but I do

69. feel that I would like to have - (.5) I don't have a CPN. My

70. biggest problem is that I don't have a CPN. I've

71. explained to my wife on several occasions that I would

72. dearly love to have a CPN for my own safety because I

73. know the seriousness of bipolar and what can happen if

74. things go wrong and that's my main worry in the back of

75. my mind, all the time.

76. Researcher: Have you ever had a CPN?

77. Alun: Yes, uhm, years ago - years and

78. years ago I was admitted - I was sectioned several

79. times, had a CPN, I was wrongly diagnosed with

80. schizophrenia at the time, back in the day in the early

81. $90 \mathrm{~s}-$ it took seven years to get a correct diagnosis and

82. uhm when I did I had the CPN for a while, I had a CPN a

83. few years ago but I've been discharged from the

84. community mental health team uhm and the difficulty is

85. getting back with the community mental health team,

86. now that I feel that I need to be back with them.

87. Researcher: And so you want to get back with

88. the community mental health team?

89. Alun: Yeah well I do feel that, I've read,

90. I've done some research on bipolar disorder, I've looked

91. at what the charity, the bipolar UK people say, and they

92. say that uhm you should have regular contact with a

93. CPN or a psychiatrist, if you've got bipolar. I don't have

94. either so I do feel that - to use a term that's been used

95. before - I'm being passed from pillar to post.

96. Researcher: One of the ways that seeing

97. somebody regularly can be looked at can be seen as

98. case management which is what a CPN would do=

99. Alun: =Yes, just on an ongoing basis. I

100. don't feel I'm in a particular crisis as such at the moment

101. but I am concerned about my health treatment not being

102. adequate should my condition deteriorate. It would take a

103. long time to be seen because in the past when I've 
104. started to feel ill it's taken several months to be seen by

105. which time I could be dead. To put it bluntly. So it would

106. be too bloody late then wouldn't it.

[Alun, Service user, Phase 1, Participant 1, lines 56-106]

The complaint, which commences at line 68 , focuses on his concern that he does not have a $\mathrm{CPN}$ as his care coordinator. This then develops into an account where an underlying contrast is made between previous good care and current poor care. In the context of his serious and life-threatening condition, this contrast amplifies his complaint about a seemingly uncaring system where there is a lack of access to a CPN through GP referral. Prior to line 68, Alun concludes his account of a largely uneventful hospital admission, with a positive evaluation of his care under primary care "doctors" (line 65). The new topicality of his complaint is introduced somewhat abruptly with the key statement "I do feel that I would like to have - I don't have a CPN. My biggest problem is that I don't have a CPN" (lines 68 to 70). Just prior to this, Alun says that he does "get the care that [he] need[s]" (line 68), which adds to the topical and evaluative contrast between care under the GP and the hypothetical care which he would like to receive if he had a CPN. The abruptness of this topic change is indicative of the high level of intentional focus which Alun directs towards this issue, at the expense of other topicalities which might otherwise be discussed within the interview. Wood and Kroger (2000) point out that what is left out of an account can often be as important as what is included. In this case, other potential topics which I as the interviewer try to raise are side-lined with a series of abrupt topic changes which work in Alun's talk to maintain a focus on the issue which is paramount to Alun, the difficulty of his not having a CPN. For instance, two further abrupt topic changes of this nature occur within this data extract. When I attempt to change the focus of the interview to past experiences of care under the CPN, which Alun initially responds to in lines 74 to 84 , he quickly brings the discussion back onto the topic of his complaint at lines 84 to 85 : "the difficulty is getting back with the community mental health team." Again, at line 101, Alun shifts the subject matter of the interview back onto his intended focus of complaint about secondary service inaccessibility. Topic shifts and topic chaining is one key way in which discourse may be constructed, and this functions in Alun's talk to emphasise the intentional focus on his complaint (Erteschik-Shir, 2007; Gee, 2010). The way in which topicality is controlled by Alun is also an aspect of interactional control of the interview (Fairclough, 1992a). Alun successfully controls the interactional nature of the interview by directing the 
topic of the interview repeatedly back onto his desired focus for the purpose of constructing his complaint based on a rhetoric of contrast between good and bad care. This rhetorical achievement serves to exaggerate a notion that the care he is receiving now, excluded from easy access to the secondary sector, is poor care, and this is the basis of his complaint.

Alun also constructs an identity for himself in this data extract as plaintiff in terms of his relationship with the interviewer. Within discourse analysis, there is a close connection between the construction of identities and the construction of relationships (Gee, 2004; Gee, 2010). Within discursive psychology for example, there is a certain agnosticism about the existence of a 'core' identity (Wiggins, 2017), which is instead understood as constructed within and dependent upon particular contexts and functions occurring within discourse and human interaction (Antaki and Widdicombe, 2008; De Fina, 2012; Edley, 2001; Gee, 2010; Widdicombe, 2008). Identities are constructed as they are situated in the nexus of human interrelations and meaningful activity, which predominantly involves language and talk (Benwell and Stokoe, 2006; Blumer, 1986; Burr, 2003; Edwards and Potter, 1992; Mead, 1934; Potter and Wetherell, 1987). Consequently, there is a close connection between identity and the relationships which occur in these meaningful interactions. Identity therefore is constructed relationally in talk in terms of the discursive behaviour which occurs between speaker and recipient. In the above data extract, Alun is positioned as a disempowered individual who has recourse to express his complaint to a third party not affiliated with the secondary services he is complaining about. An important function of complaints is their ability to counterbalance disempowering practices by challenging the practices and assumptions of the services they receive, "particularly when those practices are seen as limiting independence, restricting choice, or not listening to the service-users" (Jingree and Finlay, 2013, p.257). Finding an individual in the form of the interviewer whom he can complain to in order to counterbalance his disempowered status, James constructs his identity in talk as plaintiff in relation to the interviewer.

The notion of script formulation in discursive psychology (Edwards, 1994) is helpful in understanding the discursive structure of topic shifts and topic chaining carried out in Alun's complaint. Scripts are derived from the schema theories of cognitive psychology and may be defined as "mental representation of routinely structured social occasions" (Edwards and 
Potter, 1992, p.20). The routine nature of satisfactory care provided in the hospital and good care from the GP (lines 62 to 69) is such a script, which amplifies and legitimises his complaint when compared with the future potential disaster scenario which may occur without the care of a CPN. This potential scenario is a repeat of an event which occurred twenty years ago where he attempted suicide by jumping off a bridge:

150. Alun: Twenty odd years ago I jumped off a bridge, right, I was

151. in the treatment of a hospital. The hospital had not

152. released me but had let me go home for several days. I

153. felt unwell and I jumped off a bridge. It was a suicide

154. attempt. I don't feel suicidal now, let's get that out in the

155. open but uhm I could have died.

[Alun, Service user, Phase 1, Participant 1, lines 150-155]

Although this is not directly mentioned in the above data extract under analysis, the scenario is clearly alluded to in emotionally loaded comments such as knowing "what can happen if things go wrong" (lines 73 to 74), and the closing comment of the extract: after waiting for referral too long, he "could be dead. To put it bluntly. So it would be too bloody late then wouldn't it" (lines 105 to 106). This highly dramatic and emotively expressed situation stands in contrast to the previous script formulation talked about from lines 62 to 69 to enhance the gravity of the future potential disaster scenario and the forcefulness of the complaint.

Further rhetorical and discursive devices are employed to enhance the construction of the complaint. There is the use of prosody by way of the pattern of stress and intonation in the use of the word "do" in line 68. In the approach to discourse analysis provided by Gee (2008), such linguistic 'cues' are classed as contextualisation signals by which speakers and writers indicate, negotiate and emphasise the immediate meaning of talk (Locke, 2004). The superlative adjective, "biggest" (line 70), is also employed to rhetorical effect to emphasise the "problem" (line 70) of not having a CPN. This occurs in the phrase, "My biggest problem is that I don't have a CPN" (lines 69 to 70), which is a re-iteration of the previous phrase, "I don't have a CPN" (line 69). Gee (2008) notes how re-iteration may be used by the speaker to emphasise meaning in talk, which in this case is an upgraded second assessment (Pomerantz, 1984) of how big a problem it supposedly is not to have a CPN. 
The aforementioned topic change at line 68 also represents a change in evaluation from a positive evaluation of hospital and GP care to a negative evaluation of current, communitybased service provision due to limited service access. Access to services is an important aspect of care continuity (Durbin et al., 2004; Jensen et al., 2014; Joyce 2004; Rose et al., 2009; Sweeney et al., 2012; Sweeney et al., 2016; Tomita and Herman 2015; Weaver et al., 2017). The negative evaluation which Alun makes concerns the overall care system available to him now and his perception of the limited opportunities it provides. For example, Durbin and associates (2004) identify 'System Access' as one of three key components of care continuity, since "creating a full continuum of accessible mental health services and supports is fundamental to continuity of care" (p.293). However, its achievement remains a major challenge in many service systems (Bachrach, 1993; Durbin et al., 2004), something which is an issue for Alun, as evidenced by his complaint.

A further discursive device which is employed is the use of intersubjective corroboration of his position. Firstly, there is the reference to Alun's wife at line 71 implying her agreement and support for his predicament. The appeal to intersubjectivity here serves the function of building up the credibility of the account (Baruch, 1981; Wiggins, 2017). Later on, a further appeal to independent corroboration is made by citing the advice of Bipolar UK (lines 89 to 93), the largest UK charity providing support for people with his condition. This is a stronger attempt at intersubjective corroboration since it is also an instance of inter-textuality (Fairclough, 1992a; Fairclough, 1992b; Fairclough, 2003; Fairclough, 2013). Intertextuality, or 'heteroglossia' (Gee, 2008), concerns the ways in which discourses may refer to or allude to other texts. In this case, Alun makes a direct reference to the textual information provided by Bipolar UK on their website. Mixing of texts in this way is identified by Fairclough (1992) as one of the main ways in which discourse may engender socio-cultural change (Fairclough, 2003; Jørgensen and Phillips, 2002). Since discourses are a key driving force in sociological practice, any novel mixing of texts within discourse implies new sociological structures. Kristeva (1986) writes that intertextuality implies "the insertion of history (society) into a text and of this text into history" (p.39). In this way, the text reworks past texts and in so doing initiates a novel process of social change or a challenge to the existing social order (Fairclough, 1992a; 1992b). This theory about text can also be applied to talk in the data set. In Alun's case, the re-contextualisation of the Bipolar UK advice within his complaint about not having a CPN, 
performs the function of legitimising his complaint, and challenging the existing social order he faces in the form of barriers to service access (Dale, 1989). The kind of legitimation being exercised here is also fairly strong since it is legitimation by reference to an organisation in whom institutional authority is vested, which Fairclough (2003) describes as legitimation by authorisation.

This instance of intertextuality and legitimation by authorisation is followed immediately by another instance of this kind of rhetorical device, drawing on the discursive resource of a commonly used idiomatic expression to provide legitimacy (Drew and Holt, 1988). At line 95, Alun says that he is "being passed from pillar to post" in seeking a timely referral. In fact, Alun specifically refers to his drawing on this phrase as being an instance of intertextuality when he says that it is a "term that's been used before" (lines 94 to 95). This term is also a metaphor which is significant from an analytical point of view. Metaphors are one of the most important and widely used rhetorical devices in discourse, although they are so pervasive in talk that they often go undetected (Coffey and Atkinson, 1996; Potter, 1996). Recalling my point made in section 3.6.4, metaphors are one of the clearest examples of a discursive device which is normalised for the analyst and difficult to detect, since it is embedded within common-sense understandings which produce the meaning and effect of talk or text. Metaphors therefore represent one of the clearest examples of the analyst being discursively embedded within the culture he or she is analysing, and being constituted by the various common-sense understandings which perpetuate hegemonic fixings of meaning (Gramsci, 1971; Kincheloe and McLaren, 1994; Laclau and Mouffe, 1985). However, analytical elucidation of the use of metaphors by speakers provides an insight into the meaning of their talk, since they reveal the speaker's ways of looking at the world, their specific values and the cultural domains that are encoded within these metaphors (Coffey and Atkinson, 1996; Fairclough, 1992; Spradley, 1979).

By employing this intertextual, idiomatic and metaphorical device in saying that he is 'being passed from pillar to post', Alun is able to input into his complaint the specific values which characterise his perspective on his experiences of negotiating services, unsuccessfully as he sees it. The values associated with the term 'being passed from pillar to post' belong to a familiar scenario or script formulation in which a person is sent from one point of healthcare 
provision to another without being able to access needed treatment. In that this is a familiar scenario, the metaphor is also being used to invoke a script formulation (Edwards and Potter, 1992). The script-metaphor taps into a commonly accepted category of unfair care discontinuity and difficulty in accessing services, which also finds backing in the literature (Blane, 2014; Gofal, 2013; Heaney, 2016; Welsh Government, 2015). In sum, the term 'being passed from pillar to post' being used in this way is a combination of a variety of discursive devices: metaphor, script, idiomatic expression and intertextuality. The overall effect of this powerful, combined use of discursive devices to construct this perspective within discourse is to provide a highly persuasive and convincing account that Alun's situation is both unfair and unacceptable.

Summing up the analysis of this extract, a great variety discursive devices are used by Alun to construct his complaint and an accompanying identity-in-talk as plaintiff. This constructed complaint is directed against and conflicts with structural realities of care access which Alun berates throughout his interview. The function and implicit purpose of Alun's discourse is therefore to challenge and undermine the legitimacy of this structural impediment which involves a perceived and experienced discontinuity of care (Bachrach, 1981; Freeman et al., 2002) for Alun at the primary-secondary interface. In many ways, Alun's case is therefore an even clearer example than James' or Alex's talk of a misalignment of expectations within shifting service structures and boundaries. In the next section I will show through further analysis of talk how implementation of the Mental Health (Wales) Measure 2010 may stimulate tensions between different understandings of mental illness and recovery, leading to misaligned expectations of services and how they may be negotiated, which may precipitate greater service system complexity.

\subsubsection{2 "They didn't want to take me back because they said to me that they thought it would be a setback for me": Service boundaries or over-expectant self-referral}

Having analysed talk related to the category 'GP referral barriers', I now deal with a data extract taken from Katy's interview, related to the category 'Part 3 self-referral barriers' in Figure 6. This completes my exploration of the conceptual context for thematic discourse analysis represented in Figures 5 and 6, related to movement in both directions across the 
primary-secondary interface. Katy, whose talk I analyse in this section, has attempted to selfrefer under Part 3 of the Mental Health (Wales) Measure 2010. She is a woman in her midthirties who I interviewed at a third sector day-centre. Katy described her visits to this day centre as occasional as she suffers from a condition of social anxiety that means she often confines herself to her home. In the following extract, Katy recounts her experience of being rejected at assessment for re-admission to secondary services after her self-referral:

19. Katy: Okay. I was referred to $<<$ CMHT $>>$

20. after a long time of having depression. I lost my mother.

21. I've had cancer. At the age of 23 , I was referred to

22. $<<$ CMHT $>>$. I went through all my problems and they gave

23. me CBT. They referred me to a dietician as well because of

24. my eating problems, which was helpful, and the CBT

25. helped me come to $<<$ Third sector day centre $>>$ to get me out of the house

26. instead of shutting myself away. Then, they decided I was

27. doing well and they discharged me. Then, I became ill

28. again, but I didn't go back until... (.4)

29. I tried to cure myself first, so I went back when I was really

30. ill again because they told me I could go back. Then, I went

31. back and explained to them that I'd just come down again,

32. that I was shutting myself away again, and that it was the

33. same process over again. They didn't want to take me

34. back because they said to me that they thought it would be

35. a setback for me, but I didn't understand that because I

36. didn't know how much more set back I had to be to be

37. taken in by them. You know? It made me feel like, "I don't

38. even want to go back there anymore for the help", because

39. they made me feel like I was just not ill enough. They said

40. to me that I was contradicting myself, so I don't really want

41. to go back and be told that again.

42. Researcher: After your experience of attempting

43. to go back to the CMHT, that was a negative experience

44. for you and you feel you don't want to do that anymore?

45. You wouldn't do that?

46. Katy: I would definitely not go there again.

47. Researcher: Because it was such a negative

48. experience?

49. Katy: I'm not saying that they were bad.

50. They did help me a lot, the first time, but they told me I

51. could go back any time I wanted and that I could get help

52. from them any time I wanted, and when I went back, they

53. refused me help.

[Katy, Service user, Phase 1, Participant 4, lines 19-53] 
As a researcher it was difficult for me not to make the mistake of performing under-analysis through taking sides with this participant (Antaki et al., 2003). Focusing on self-reflection, I found myself to be sympathetic to Katy's plight in having been originally told that she could refer herself back to services (line 30), but ultimately having this referral repudiated. She reports the circumstances surrounding this rejection as involving the rationale that she should not be accepted back into the CMHT as this would be a "setback" for her care (line 35). The implications of the lines following this report are that she has been deterred from further helpseeking in this way (Robertson, 2016), and furthermore, that a perceived broken promise about self-referral has led to a complete breakdown of trust in secondary services and associated professionals (lines 37 to 41). I found the description of re-admission as a "setback" (line 35) particularly concerning since the implication of this is that access to specialist treatment would somehow be detrimental to her health and wellbeing. However, it was important for me to remember that this is Katy's positioned account of what happened should not be interpreted first and foremost in a literal way as a direct representation and report of reality (Denzin and Lincoln, 1994; Dingwall, 1997; Fine, 2002; Gubrium and Holstein, 2004; Schwandt, 2000; Wood and Kroger, 2000).

According to this interpretivist understanding of talk, the account functions in such a way as to create sympathy in the listener or reader for Katy and for her version of events. In my recollection, the interview as a whole was very much an encounter of this nature. I found it difficult not to get drawn into Katy's perspective and lose the objectivity of my position as an academic researcher, especially given the highly effective use of discursive and rhetorical devices Katy uses in constructing her account. For instance, Katy employs an emotion category / report in lines 37 to 39 to set her account towards one which will invoke sympathy in the listener: "It made me feel like, "I don't even want to go back there anymore for the help", because they made me feel like I was just not ill enough" (my emphasis added). This emotional report is enhanced by the additional rhetorical devices such as re-iteration of the phrase "made me feel" (line 37 and line 39), intersubjective corroboration with the phrase, "You know" (line 37) and, more subtly, the use of passive voice to describe her relation to the emotional category: "made me feel" (line 37 and line 39). This to an extent expunges her of agency in relation to the emotional state, and therefore her responsibility for it. This enhances the idea that the difficulties she has encountered are due to real and objective factors which would necessitate 
such an emotional response since they are unfair. This version therefore brackets out an alternative view that service structures and boundaries at the interface of primary and secondary care are not unfair but are the result of particular circumstances surrounding her self-referral, and the particular nature of service provision she encounters. In this case, the situation is not so much about service implacability creating an unfair situation but a misalignment between her view of how services should behave and the realities of how they function given recent service changes. The account is also another complaint, which is another good example of how this mode of discourse is often used by individuals who perceive themselves to occupy a disempowered position within society (Jingree and Finlay, 2013). The effect of her account is therefore to paint a picture of her as an unfortunate, innocent and powerless victim of discriminatory treatment at the hands of an implacable, impersonal and uncaring medical organisation.

The data extract is completed with a final disclaimer (Scott and Lyman, 1968) of the idea that she is constructing a complaint entailing a very negative portrayal of the CMHT assessment team: "I'm not saying they were bad" (line 49). The statement that they are not 'bad' clearly contradicts with the gravity of the atrocity story she constructs (Baruch, 1981; Mishler, 2005). By employing a disclaimer in such a way, Katy distances herself from her position of biased stake in the situation - as plaintiff constructing a complaint (Edwards and Potter, 2001; Potter, 1996; Wiggins, 2017). This discursive device therefore adds a sense of objectivity and credibility to her negative construction of the CMHT. This is referred to as 'stake inoculation' (Wiggins, 2017) where the speaker cloaks their investment in the particular construction they are achieving, overlaying it with the gloss of objectivity, and investing in the account a greater element of credibility.

However, wishing to maintain my researcher's stance of studied neutrality, I considered the alternative view that it might be the case that the assessment team had made a justifiable decision in choosing not to accept Katy back into secondary services at the point of assessment. It is not necessarily the case that treatment within secondary care is the best and right option for her, and much of the official literature associated with the Mental Health (Wales) Measure 2010 represents the view that recovery should increasingly be pursued at the primary level, associated with a greater degree of empowerment and self-reliance on behalf of the individual 
(Lester et al., 2004; Lester, 2006; WHO, 2008). In line with this, a higher threshold for access to specialist services has been established by Part 3 of the Measure (RCPsych, 2014), corresponding to the prudent healthcare principle of focusing resources on individuals with the greatest need (Aylward et al., 2013).

As I have argued in this chapter and in relation to formation of the theme 'Misaligned expectations in negotiating transforming services', such a shift of emphasis and related service transformation may lead to incongruence between patient or professionals' views and the new behaviour of services. This incongruence is exacerbated by the potential for service transformations to produce unexpected and even undesirable outcomes, or 'waves of consequences' (Hannigan and Coffey, 2011; Rittel and Webber, 1973), which are then received poorly by individuals.

If it is the case that the decision not to accept Katy back into secondary services is in her best interests, then the difficulty here, which Katy expresses, is that she is unable to accept the workings of the new system. This is because her expectation is that in self-referring she will automatically be accepted back into services. This however, is not necessarily a realistic expectation for the self-referrer to have. Given that Katy has this expectation, a fundamental impasse exists for her, regarding acceptance back into specialist mental health services, since it is the case that this is dependent on a diagnosis of SMI by psychiatric experts. Psychiatrists are the sole profession with the power to make a diagnosis of mental illness, in conjunction with the use of diagnostic manuals such as the DSM-V and the ICD-11 (APA, 2013; WHO, 2017). In this regard, a great deal of the determination of the appropriate level of treatment is beyond the power of the service user, since they do not have the power to self-diagnose. Expert psychiatric diagnosis is a key determinant of the accessibility or inaccessibility of specialist services, regardless of however much empowerment may be facilitated by a recovery ethos or by policies designed to implement this (Bonney and Stickley, 2008; Keetharuth et al., 2018; Leamy et al., 2011). In a sense, this is what Katy is up against, being newly empowered by the option of self-referral under Part 3 of the Measure, though she interprets this circumstance as a needless and unjustifiable expropriation of her care. 
According to this understanding, there is a tension between the new behaviour of services due to implementation of the Mental Health (Wales) Measure 2010 and the expectations inherent in the talk of participants. On the one hand there is the impetus towards greater empowerment and self-determination inherent in Parts 1 and 2, underlined by recovery values (Welsh Government, 2010a; 2013; 2014c). Along with this, a higher threshold for access to specialist services is established by Part 3 of the legislation (RCPsych, 2014), in line with prudent healthcare principles (Aylward et al., 2013). This tension, manifest in Katy's discourse, can be seen as a further complex effect emanating from implementation of the Measure, or another 'wave of consequence' which is involved with service transformation. What might help to reduce this incongruity between expectations and changing service behaviour would be provision of a better explanation of the nature of the self-referral option for Katy, though this does not seem to have occurred. Instead, Katy's talk indicates that she has now developed a negative view of services which she wishes to avoid (lines 40 to 41 and line 46), which could also be viewed as a further wave of consequence emanating from implementation of the Measure.

All of this is problematic in terms of the successful implementation of Part 3 of the Mental Health Measure. The idea behind this part of the legislation was that there would be a safetynet for increased numbers of people being discharged to primary care (RCPsych, 2014; Welsh Government, 2014c). At the same time, individuals would be given greater empowerment with regard to their healthcare, in line with recovery values, which would include a new power to self-refer directly back to specialist services under Part 3 of the Measure (Bonney and Stickley, 2008; Leamy et al., 2011; Welsh Government, 2013; 2014c). However, this new power does not involve the ability to credibly diagnose and determine the presence of mental illness. Consequently, the option to self-refer faces a major boundary which ensues from it still being the case that access to specialist services is essentially dependent upon professional psychiatric diagnosis.

Whilst this conflict has led to a misalignment of expectations for Katy, related difficulties have been the subject of some criticism of Part 3 of the Measure and its implementation, which has not been viewed as working well by some commentators. For example, the Royal College of Psychiatrists' post-legislative scrutiny of the Measure identifies difficulties in establishing 
agreement about who is a 'relevant patient' for secondary services (RCPsych, 2014). The presence of this disagreement is borne out by some statistical evidence provided in Welsh Government reports. According to the Welsh Government's 'Duty to review interim report post-legislative assessment of the Mental Health (Wales) Measure 2010' (2014c), $n=394$ people were accepted back into secondary services out of 909 people who undertook selfreferral, during the period April to December 2013. This leaves more than 50\% of self-referrers under Part 3 encountering rebuttal from services on the basis that there is not sufficient presence of mental illness, which would presumably conflict with the view of the self-referring patient trying to access services.

Supporting the idea that there is disagreement or misalignment between the views of selfreferrers and professionals determining acceptance or non-acceptance back into secondary services, in my conceptual data analysis, six participants said that they had not seen any successful self-referrals back to secondary services or that they had seen very few. No other participants spoke about seeing significant numbers of self-referral, and even the few selfreferrals that were reported in the data set required substantial third sector support for them to be facilitated (see concepts 'Few or no successful self-referrals' and 'Third sector support needed for self-referral' in Figure 6). If it is the case that there is a disharmony between service user expectations of being accepted back into secondary services and the professional opinions of assessors, this is an issue for the functioning of Part 3 of the Measure.

Furthermore, James, later on in his interview than the data extract provided above, describes a situation where other service users he knows who were attempting self-referral were rebuffed by CMHT receptionists and told to go back to their GP.

653. James: They knock at their door, when

654. they're getting to the receptionist, they're told that if they

655. have a problem, they have to go to the GP.

[James, Service user, Phase 1, Participant 8, lines 653-655] 
Within the literature and within my analysis of the data set at both conceptual and discursive levels therefore, there is evidence of the presence of misaligned expectations about the accessibility of specialist support through self-referral.

Underlying this I would argue that a difficulty surrounds establishing whether or not someone is mentally ill, and to what severity, because of problems related to clarifying the nature of mental illness. Schools of thought such as antipsychiatry and critical psychiatry have focused on this difficulty and a lack of consensus about the nature of mental illness, which is often contrasted with physical illness (Double, 2009; Rogers and Pigrim, 2014; Szasz, 1974a). In the absence of agreement on this issue, the question of presence and degree of mental illness must ultimately rest on the opinion of professional psychiatrists. Arguably, this goes against the grain of a recovery-ethos underpinning the Mental Health (Wales) Measure 2010, seeking to implement increased service-user empowerment. This tension, and its unresolved nature, feeds into escalating complexity and 'waves of consequences' inherent in the misaligned expectations which people express through talk about negotiating the boundaries of transforming service structures.

\subsection{Conclusion}

In this chapter, I have presented analysis of the theme 'Misaligned expectations in negotiating transforming services' which has been generated by qualitative analysis of data extracts from the data set. This qualitative analysis has combined conceptual analysis with discourse analysis of participants' talk. Analysis has shown that service structure changes, in part influenced by implementation of recovery-based services by the Mental Health (Wales) Measure 2010, have led to the complex systems effect of misaligned expectations in negotiating the primarysecondary interface boundary. Analysis of talk in this chapter has shown that, in a number of professional and service user contexts, expectations diverge from the actual behaviour of services in a way which involves an escalation of service system complexity. This to an extent is the result of the intention under the Measure for treatment of SMI to be increasingly located at the primary level. I have indicated that such misalignment rests partly on difficulties surrounding responses to these service changes, and at a deeper theoretical level, upon the enigmatic and contested nature of mental illness. Specifically, the phenomenon of misaligned 
expectations can be considered to be a complex effect or 'wave of consequence' emanating from a solution, in the form of the Measure, to the 'wicked problem' (Rittel and Webber, 1973) of implementing recovery-based mental health services in Wales (Welsh Government, 2010a; 2014c). This develops my argument that the Mental Health (Wales) Measure 2010, introducing service transformation, has served to exacerbate existing service complexity and fragmentation, which is seen through analysis in this chapter in the form of misaligned expectations about shifting service structures and interfaces.

In the next findings chapter, I describe the theme, 'Competing versions of recovery'. Based on the theoretical understanding of recovery introduced in section 2.6, which provides a context for analysis, I argue that competing versions of recovery are constructed in participants' talk through instances of colonisation of the concept by a variety of external influences and agendas. A key division between competing versions of recovery occurs between primary care and the third sector. On this basis, I argue that this service interface is demarcated by competing recovery versions. In this and a variety of service contexts, competing versions of recovery contribute to escalating service complexity and fragmentation, with negative implications for care continuity. 


\section{Chapter 5: Competing versions of recovery constructed in participants' talk about service experiences}

\subsection{Introduction}

In this chapter I describe the theme, 'Competing versions of recovery', constructed in participants talk about their experiences of services. My argument based on my analysis of data collected for this study develops the theme of this chapter and proceeds on the following lines. 'Recovery' is identified as a 'polyvalent concept' (Pilgrim, 2008; Pilgrim and McCranie, 2013) and a 'floating signifier' (Jørgensen and Phillips, 2002; Laclau and Mouffe, 1985) in participants' talk. There are many disparate and potentially competing versions of recovery since it is a contested concept lacking clarity which may be appropriated in different ways. Additionally, as I have already outlined in review of the literature in section 2.6 , recovery is a highly colonised concept (Barker, 2003; Mind, 2008). Agendas and interests external to recovery theories are used by agents to colonise the concept, constructing new, potentially disparate and competing versions of recovery.

In addition to this, I argue that the Mental Health (Wales) Measure 2010 represents the introduction by policy-makers of a particularly colonised version of recovery, under a neoliberalist ideological agenda, into the service system. Individuals may be socialised into accepting such a recovery approach, which may lead to impediments to their care progression, since it is a very narrow interpretation of recovery (Morrow, 2013; Ramanuj et al., 2015; Recovery in the Bin, 2019). I also focus in particular in this chapter upon the service interface between the third sector and primary care. I argue that competing versions of recovery constructed by individuals and groups within these two different sectors impedes potential collaboration and cross-boundary care continuity (Adair et al., 2005; Sweeney et al., 2016; Uijen et al., 2014) between these service sectors. This has further implications for the developing picture in this thesis of fragmented services characterised by escalating complexity.

Analysis also leads me to view colonisation of the recovery concept as related to its polyvalency, both exploiting this nature and encouraging it, since colonisation is a pervasive driver for the inception of new, competing versions in Welsh mental health services. Different 
versions of recovery are constructed in different occurrences in which particular instances of colonisation of the concept occur, as my analysis will show. In this sense, I saw my task in this chapter as qualitative analyst to identify and analyse such instances of recovery constructions as they occur in talk, contributing to my argument supporting the theme 'Competing versions of recovery'. On this basis, I was then able to make inferences about the impact which this scenario might have for the behaviour of services. I now describe this analysis in subsequent sections of the chapter.

\subsection{Construction of the theme 'Competing versions of recovery'}

The theme 'Competing versions of recovery' was generated from two stages of analysis involving concept mapping and discourse analysis, as described in the methodology chapter. The theme sits across three categories, 'Recovery approaches and constructions', 'Third sector care and support', and 'Third sector collaboration with primary care'. These categories and related concepts are shown in turn as concept maps within this chapter.

\subsubsection{Recovery approaches and constructions}

This category consists of data related to the various ways in which participants talked about recovery approaches to mental healthcare. Figure 7 shows the concept map which I built out of coded analysis of participants' talk on recovery. The various concepts in the following map therefore represent specific versions of recovery talked about by participants: 
Figure 7: Recovery approaches and constructions

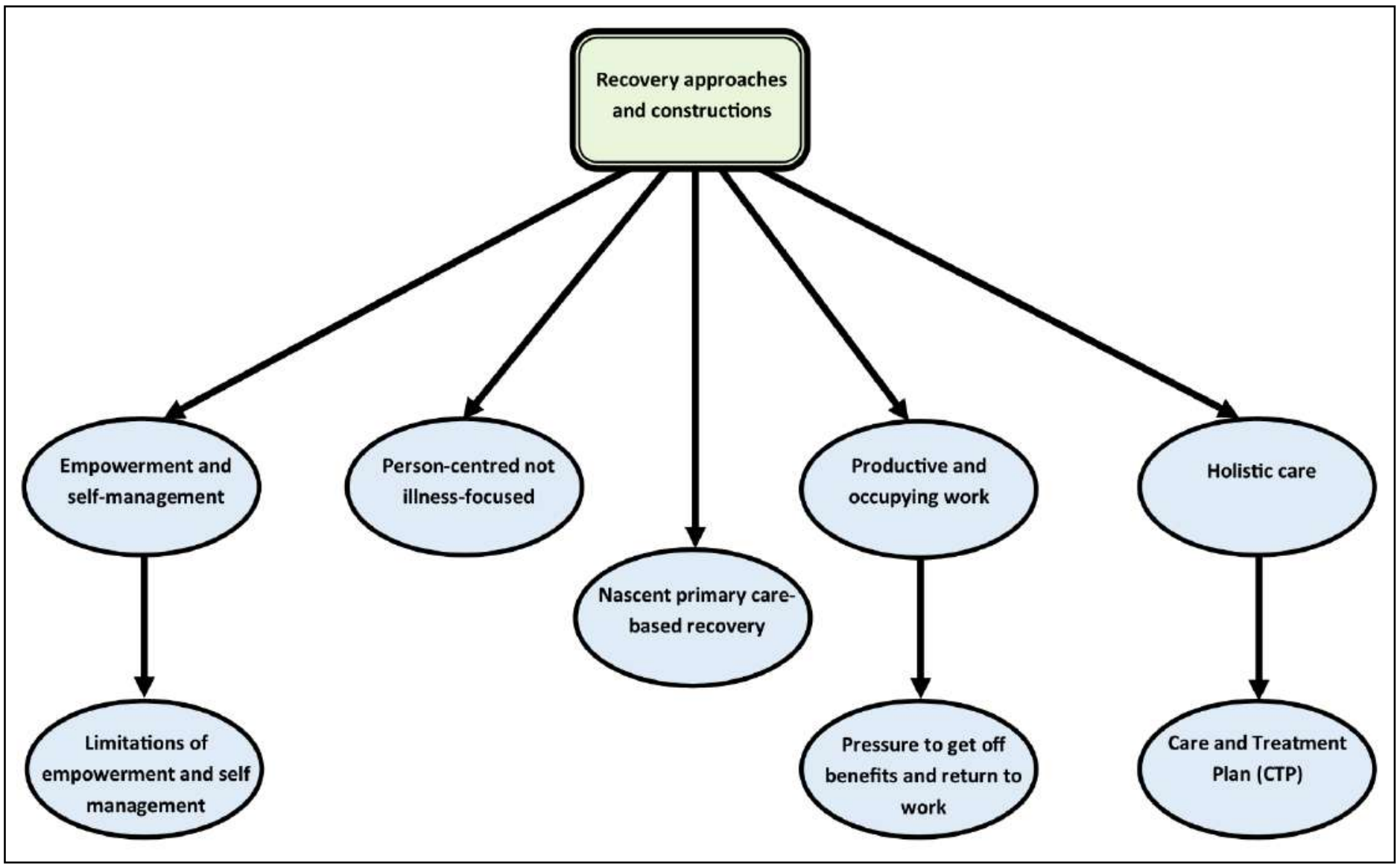

Analysis in this chapter will show these recovery versions may be seen as competing when compared to one another conceptually and theoretically. The concept map above indicates that talk among participants coalesced around versions of recovery which may be labelled as 'Empowerment and self-management' $(n=10)$, having a person-centred rather than an illness or deficits focus $(n=4)$, 'Holistic care' $(n=3)$, finding 'Productive and occupying work' ( $n$ $=4)$, and 'Nascent primary care-based recovery' $(n=3)$. I discuss the latter of these concepts in relation to GP participants' talk in section 5.4.2 where I argue that new versions of recovery are being constructed based on the strengths of primary care. Three of these concepts were qualified by related concepts identified in the data set. Finding 'Productive and occupying work' was qualified by the perception that some of the pressure to return to work was guided less by a recovery ethos and more by socio-economic pressure rooted in a neoliberalist agenda (Mind, 2008; Pilgrim, 2008; https://recoveryinthebin.org/). This concept is labelled 'Pressure to get off benefits and return to work'. One participant, Bethany, a manager of a third sector day centre, relates in her talk about the concept of 'Holistic care' to a particularly relevant 
instance of this in the form of the 'Care and Treatment Plan (CTP)'. This is a care planning approach in Welsh services, which claims to focus comprehensively, and in this sense holistically, on the service user's life, broken down into eight areas (Welsh Government, 2014c; 2015; 2016).

The version of recovery constructed by most participants $(n=10)$ was 'Empowerment and self-management'. Six of these were service users, two were third sector workers and two were GPs. Relevant to this recovery concept, I present in the following section two data extracts taken from a research interview with one of these participants, Dylan. This individual focuses in his interview primarily on a self-management version of recovery, and he is one of the most eloquent purveyors of this version in the data set. However, despite the apparent commitment to this single version of recovery based on self-management, my analysis shows that multiple competing versions of recovery are constructed within Dylan's talk. Dylan's verbal dexterity, experience as a support worker and high level of awareness about the nature of mental illness and services results in his talk being an exemplar of the polyvalent nature of the recovery concept, once this is elucidated by qualitative analysis. Analysis of Dylan's talk is therefore an appropriate place to start for my argument supporting the theme 'Competing versions of recovery'.

\subsection{2 “It's a bit ambiguous that word, isn't it?" Recovery, a polyvalent concept generating competing versions}

Dylan is a service user with bipolar disorder in his fifties and also an Expert Patient (DoH, 2001a; Hopton, 2006; Lorig et al., 1993) providing support for people with SMI in a variety of third sector settings. Dylan's talk combines his direct service user experience with his knowledge of day-to-day work with service users at the interface of primary and secondary care. As a service user, Dylan offers his views grounded in the lived experience of having mental illness and receiving care and treatment for this condition.

The following data extract follows on from my inquiry about the nature of the self-management approaches he advocates and teaches. Dylan responds by focusing on this topic at some length, and then continues to make it the major focus of a relatively long interview, lasting an hour 
and six minutes. Self-management is clearly an important subject for Dylan given the degree of his topical focus on it throughout the entire interview, as can be seen in the following data extract:

160. Dylan: Self-management is the key to recovery,

161. and I don't know, it's a bit ambiguous that word, isn't it because

162. you know I'm fifty-two now, thirty years on from when I was first

163. diagnosed and here I am now and I'm still managing this

164. condition. So I cannot pretend to say to you that I'm symptom

165. free because I'm not. And if took my, erm - (.3) what's the right

166. word - and if I took my foot off the clutch then I'd be stuffed

167. really - I've got to make sure that every night I get my sleep

168. properly. It's a holistic approach. There's nothing difficult

169. about this really. It's about eating properly, sleeping properly,

170. exercise - something I struggle with to be honest; connecting

171. with people, engaging with people, routine, but also I think for

172. me - (.) work - (.2) I find a lot of meaning and purpose in work, as

173. well, and it really helps to keep me well as well. Appropriate

174. work, mind, and that's been a struggle to find appropriate

175. work, in all of that as well, erm, but it's like a jigsaw I guess,

176. it's making sure all the pieces are together and I will say to

177. you I still take medication, I still take lithium a mood stabiliser,

178. albeit a very small amount now. I see the value of medication

179. stabilising people but it isn't all about medication, I think the

180. health service needs to practice what it says in theory but it

181. doesn't do it in reality, and that is a whole person approach,

182. you know.

[Dylan, Senior third sector support worker, Phase 2, Participant 4, lines 160-182]

A number of statements within this data extract are consistent with a self-management version of recovery. Most obviously, there is Dylan's opening statement, "Self-management is the key to recovery" (line 160). He then goes onto describe himself as "still managing this condition" (lines 163 to 164) thirty-two years on from when he was first diagnosed (lines 162 to 163). The use of the metaphorical phrase "foot off the clutch" (line 166) implies a sense of remaining in control of one's life as one would do so with a vehicle. This form of speech is a version of the commonly used phrase "take your foot off the pedal" meaning "to rest, relax or do nothing" (Rundell and Fox, 2010). As has already been noted in previous chapters, metaphors encode people's values and cultural embeddedness revealing their ways of looking at the world (Coffey and Atkinson, 1996; Fairclough, 1992b). Dylan's talk here can be interpreted as encoding the 
meaning that he needs to remain vigilant and diligent in maintaining his recovery and mental health. It also implies that he maintains control over his life and recovery through proactive use of self-management approaches, employed on a daily basis. That this has already been continuing for the past thirty-two years implies that it is likely Dylan will need to continue with diligent application of this self-management approach for the rest of his life.

Dylan follows this introduction to his self-management approach to recovery with a series of statements describing some of the practical details of this approach. Dylan must make sure that he gets enough sleep every night (line 169). He must attend to a proper diet (line 169), exercise sufficiently (line 170), maintain a good routine (line 171), and engage in appropriate and meaningful work (line 172). All of these activities can be seen as components of a selfmanagement approach to recovery. For example, they are similar to the components of the 'Wellness Recovery Action Plan' (WRAP) created by Mary Ellen Copeland (2000), which entails a practical, person-centred plan for self-management to develop personal empowerment and recovery (Davidson, 2005; Pilgrim and McCranie, 2013). The first part of WRAP is a personal Wellness Toolbox (Copeland, 2000), which is a list of resources for supporting getting a good night's sleep, having a good diet and exercising. Another recovery model tool which can be considered to be relevant to a self-management version of recovery is the Recovery Star (McKeith and Burns 2010; Pilgrim and McCranie, 2013). This focuses on combined development of ten separate areas of the client's life including work, living skills, physical health and self-care (McKeith and Burns, 2010), which are relevant to Dylan's practical approaches. In Wales, the CTP approach can be considered to be another example of a recovery self-management version, given the "requirement under the Measure for care plans to focus on recovery" (Welsh Government, 2015, p.38). In the CTP, eight specified areas are entailed in this recovery-oriented, care planning approach including personal care and physical wellbeing, and work and occupation (Hafal, 2012; NHS Wales, 2018), features which are also comparable to Dylan's description.

Underpinning the CTP approach is a self-management approach to recovery for people in secondary services related to various life-management goals entailed in this care planning approach, where the CTP is signed and agreed between service user and care coordinator (Welsh Government, 2014c; 2015; 2016). Instead of a dependency on services, the focus of 
such empowerment and self-management versions of recovery as WRAP, the Recovery Star and the CTP approach is upon the person's strengths and inner resources, instead of deficits and illness, whose cultivation allows the individual to grow beyond the limitations of mental illness. William Anthony's (1993) heavily cited statement on the "guiding vision" (p.521) of recovery expresses this central idea:

"Recovery is described as a deeply personal, unique process of changing one's attitudes, values, feelings, goals, skills, and/or roles. It is a way of living a satisfying, hopeful, and contributing life even with limitations caused by illness. Recovery involves the development of new meaning and purpose in one's life as one grows beyond the catastrophic effects of mental illness."

(Anthony, 1993, p.527)

This statement, which I used in both interview schedules to introduce the idea of recovery to participants, has a number of implications, but the final idea of "growing beyond the catastrophic effects of mental illness" (Anthony, 1993, p.527) suggests a focus on personal development and empowerment rather than a pre-occupation with cure. One of the central ideas of recovery in mental health is that cure might not be possible. This is a clear alternative to more traditional definition of recovery as amelioration of symptoms and a return to normal functioning, or cure in the conventional sense (Davidson et al., 2005). Dylan references the idea of recovery-as-cure from illness when he describes recovery as "a bit ambiguous" in line 161 meaning that whilst the term is conventionally understood as cure, this is not his understanding of it. Rather, recovery necessitates a perpetual, lifelong journey of daily, vigilant self-management, as indicated by the foot off the clutch metaphor (line 166). ${ }^{10}$ Thirty years on from diagnosis, he is still managing his condition (lines 163 to 164), and he cannot pretend that he is symptom free (lines 164 to 165). Medication is a part of this management process but it is not a total panacea since recovery "isn't all about medication" (line 179). This also adds to

\footnotetext{
${ }^{10}$ One strand of the recovery movement in modern mental healthcare can be associated with the conventional understanding of recovery from illness, meaning a partial or even total remission of symptoms (Mind, 2008; Pilgrim and McCranie, 2013). This view replaced the formerly pessimistic, Kraepelian prognosis associated with mental illness, reflected in stigmatising social attitudes and public policies emphasising social control rather than therapeutic treatment (Foucault, 1973; Mind, 2008; Rogers and Pilgrim, 2014). However, the wider focus of the recovery movement was on a more radical agenda with a more generalised emphasis on therapeutic hope and optimism (Lester and Gask, 2006; Pilgrim and McCranie, 2013). Within this understanding, recovery can occur even though symptoms persist, since the focus is less about alleviation of symptoms, and more about reducing the degree to which symptoms interfere with a person's life (Repper and Perkins, 2003). Even though a reduction in symptoms is seen as a positive and helpful aspect of recovery, this may not be a linear process since both growth and setbacks can be encompassed in the recovery journey (Anthony 1993).
} 
the development in Dylan's talk of the idea that recovery is not about cure, or being 'fixed' by medication, but an ongoing process of daily self-management.

Further analysis of the above data extract indicates an additional version of recovery constructed in Dylan's talk, grounded in the concept of holistic care. This version of recovery is supported by various theoretical approaches to recovery (Amering and Schmolke, 2009; Gilburt, 2013; Shepherd et al., 2014), as well as the CTP approach (Welsh Government, 2010a; 2014c; 2015). In line 168, Dylan says, "It's a holistic approach", identifying recovery with holism. In addition to this, the discursive device of the metaphor "jigsaw" (line 175) is used by Dylan to further enhance his constructed association of holism with recovery. The phrase, "making sure all the pieces are together" (line 176), works to support his claim that holism provides the means and comprehension according to which to identify and assemble the necessary pieces of his recovery. These include the various, practical components of his selfmanagement approach to recovery which are provided above. This also implies that there is an intrinsic connection between the self-management version of recovery Dylan is chiefly describing and the holistic recovery version he also constructs in his talk.

An holistic understanding or version of recovery is potentially problematic theoretically speaking due to problems associated with conceptions of 'holistic' healthcare. This concept is arguably unclear and contestable in a similar way to the polyvalent recovery concept (Clarke, 2003). 'Holistic' healthcare is another nebulous term with a variety of meanings not just confined to its application in the form of mental health recovery (Gordon, 1990; Lindquist et al., 2018; Ventegodt, 2016). Its manifestation as a recovery version may also be regarded as one instance where the recovery concept has been colonised by an external approach to healthcare, since holism did not occur in the earliest theories and ideas about recovery (Anthony, 1993; Deegan, 1988; Deegan, 1993). Holistic care has particularly been appropriated by the nursing profession and is frequently touted as the linchpin of its claim to autonomy from medicine (Clarke, 2003). However, key difficulties are related to the challenge of finding a clear statement or definition about the central ideas of holism (Patterson, 1998; Phillips, 1977). Indeed, it could be argued that holism is intrinsically hard to articulate from a theoretical point of view, since its central thesis is that nothing true may be said about any aspect or component 
of anything considered holistically unless this also simultaneously refers to that entity's whole (Clarke, 2003).

The difficulty in combining the two contestable concepts of recovery and holism is reflected in Dylan's language. Dylan's identification of the two concepts in the phrase at line 168, "It's a holistic approach", is an anaphoric reference ${ }^{11}$ which amounts to a fairly oblique way of combining these two concepts discursively. Gee (2010) points out that speakers choose subjects and their predicates strategically in the construction of discourse determining the constructed version of reality they are producing, and consequently the way listeners view what is being talked about. In view of this, Dylan's identification through anaphoric reference of recovery as subject with holism as its predicate can be seen as a tactic to achieve a contentious connection between these two imprecise concepts of recovery and holism, and a way of constructing an holistic version of recovery.

Despite these theoretical difficulties, which are reflected in Dylan's talk, holistic healthcare is frequently associated with recovery in the literature on more recent theories on recovery. The label of holism often features within a person-centred recovery approach aimed at achieving personal integrity and wholeness (Amering and Schmolke, 2009; Gilburt, 2013; Shepherd et al., 2014). Holism is one of the ten dimensions of recovery in the SAMHSA consensus statement (Amering and Schmolke, 2009; SAMHSA, 2004). It can also be applied to an understanding which seeks an integration of or parity of esteem between mental and physical health and care, where the person is viewed holistically in the sense that both physical and mental aspects are encompassed (Health and Social Care Act, 2012; WHO, 2008). At the same time, it may be related to self-management approaches since a person's life subject to this management is multi-faceted. One example of this combination of self-management and holistic versions of recovery is the CTP approach in Wales. This care planning approach involves a framework for self-management which is also holistic in the sense that eight separate areas encompassing the entirety of a person's life are covered by the approach (Welsh Government, 2011a; 2014c; 2016). In this vein, the 'Duty to review final report' on the Measure

\footnotetext{
11 'Anaphoric reference' is where the meaning of the pronoun depends upon an antecedent expression (Halliday and Hasan, 2014). In this case, the pronoun 'it' is referring to 'recovery'.
} 
(Welsh Government, 2015) states that "the primary intention of the Measure was to provide... all individuals, who were receiving secondary mental health services, with a care coordinator and a proportionate and holistic care and treatment plan" (p.6).

Another version of recovery may be discerned through analysis of Dylan's talk, based on the notion of a critique of services (Pilgrim and McCranie, 2013). Whilst the self-management version of recovery sits with his role as third sector support worker and Expert Patient providing support for clients, this recovery version is also grounded in his lived experience as a service user, related to traumatic experiences he recounts about mistreatment at the hands of services. The critique of services version of recovery is first hinted at by Dylan in lines 179 to 181 in the above extract where he makes the criticism that services do not always carry out in practice and reality what they say should be done in theory. Dylan then expands on this critique later on in the interview:

435. Dylan: What I

436. see is you have a system in place locally where you have a

437. plethora of, well not a plethora, you have mental health

438. professionals just not doing their jobs really. And dealing with

439. very vulnerable people they aren't always predisposed to

440. actually question or challenge what level of care they're not

441. receiving or should be receiving. So sorry, it sounds a bit

442. negative.

443. Researcher: No, you can be as positive or negative as you

444. want.

445. Dylan: I mean I forgot to say there are nice people, there are

446. nice people obviously. But I think what you find is, and

447. working in West Wales, what I've found working over there is

448. you have loads of service users who will not engage with the

449. services and actually, who will pay lip service, turn up to the

450. odd appointment, or actually get their support from a peer

451. support group or self-help group or friends or acquaintances

452. or an art group or something, and will deliberately avoid

453. getting too entangled with services because they don't find

454. them very therapeutic really. And it's sad really because I

455. think the NHS think's they're delivering a service, quality

456. service and they're not and people are finding their support

457. outside the service from elsewhere actually, recently as well.

458. In the past we've said those of us with mental health

459. problems, that when you go and see a psychiatrist they ask

460. how you are you just say well I'm fine because you're not 
461. going to tell them how you really feel because they might give

462. you even more medication then so I mean it's best to keep

463. quiet really isn't it. Just tell them you're fine really thanks.

[Dylan, Senior third sector support worker, Phase 2, Participant 4, lines 435-463]

This extract can be seen as a complaint utilising an Extreme Case Formulation (Pomerantz, 1986) to discursively construct a recovery version related to a critique of services. Dylan paints an extreme version of services in which "mental health professionals [are] just not doing their jobs really" (lines 437 to 438), whilst service users, or "very vulnerable people" (line 439), pay "lip service" to them (line 449) and do not really engage with services. Rhetorical devices are employed to enhance the complaint such as the extreme adverb of degree, "very" (line 439), to describe vulnerable people with mental illness. Additionally, there is the reiterated description of mental health professionals as "nice people" (lines 445 and 446) which has the effect of a disclaimer for his investment and stake in this damning indictment of mental health services. Of similar effect is the apology he provides at lines 441 to 442 that his account sounds "a bit negative." As with Katy's account in the previous chapter, such disclaimers achieve a certain degree of inoculation from the speaker's stake in their complaint, thereby adding the gloss of objectivity and credibility to the account. Here, this contributes to Dylan's construction of a recovery version based on a critique of services (Pilgrim and McCranie, 2013).

The connection between a self-management version of recovery and a critique of services is developed by Dylan throughout the course of his interview, culminating in the above data extract. In many ways, this view of services and basis for recovery creates the theoretical context in which a self-management recovery approach may be advocated. If services are unhelpful, or even coercive and iatrogenic, and should be avoided, the alternative path to recovery is self-management. This is the implication of the co-occurrence of these two recovery versions in Dylan's talk, based on self-management and a critique of services respectively. This connection is also made in the literature. For instance, in their survey of the recovery field, Pilgrim and McCranie (2013) identify one of the four major approaches characterising recovery as a critique of services. According to this theoretical perspective on recovery, an emphasis on empowerment and self-management is related to the sense of recovery as a critique of services. Recovery models may often "emphasise choice, empowerment, reform and the upending of the more customary clinician-patient dynamic" (Pilgrim and McCranie, 2013, p.51) which traditionally places the clinician in a paternalistic role. A reorientation of the power dynamic 
between patient and clinician is thus achieved by establishing the patient as an active participant able to make choices in a collaborative relationship with the health professional (Lester, 2006). The connection between these two aspects of recovery is also constructed in Dylan's talk, as can be seen from the two data extracts provided above which span much of the interview. In the first data extract he focuses on self-management and empowerment. In the second data extract, he focuses on a critique of services implying the need to essentially avoid genuinely engaging with services. Interestingly, avoiding services is actually a component of some multi-dimensional models of care continuity provided in the literature (Burns et al., 2007; Rose et al., 2009; Sweeney et al., 2012; Sweeney et al., 2016). In sum, both in Dylan's interview and in certain theories of recovery, self-management and a critique of services go hand in hand (Pilgrim and McCranie, 2013; Repper and Perkins, 2003).

I would argue that, the self-management recovery version constructed in Dylan's talk, which is coupled with a critique of services, is a less colonised version than the kind of self-sufficiency recovery approach implemented by the Mental Health (Wales) Measure 2010, which does not involve this element of services critique from the point of view of the service user. In section 2.6, I argued in my literature review that the Measure can be associated with a neoliberal consensus of certain policy-makers on recovery, which is colonised by external agendas to cut welfare get and people back to work (Braslow, 2014; Mind, 2008; Morrow, 2013). In the case of the Measure, I argue that the underlying driver behind promotion of self-management in the context of primary care is a neoliberalist policy concern with financial constraints and efficiency, underlined by prudent healthcare principles. Dylan's self-management version of recovery, on the other hand, is rooted in a critique based on an emancipatory agenda to liberate the individual from subjection to devaluing and disempowering services that may be coercive or even iatrogenic. It is less colonised since it is based on an agenda for emancipation rooted in the lived experience of the service user, which may be related to some of the original conceptions of the radical idea of recovery (Deegan, 1988; Repper and Perkins, 2003). A key distinction may be made therefore between a bottom-up self-management approach to recovery rooted in the lived experience of the service user combined with an emancipatory agenda to liberate people from coercive services, and a top-down self-management recovery version (or 'neorecovery') which is colonised by a neoliberalist ideology (Recovery in the Bin, 2019). 
Three main versions of recovery have been identified so far by analysis of Dylan's discourse: recovery as self-management, recovery as an holistic approach and recovery as a critique of services. In addition to these three main versions which Dylan advocates in his talk, there are an additional three peripheral versions of recovery which are in Dylan's account though they are not centrally advocated by him. First, there is the medicalised notion of recovery-as-cure, which is acknowledged by Dylan as an alternate or competing version he does not endorse. This is described in lines 164 to 165 in the first data extract where Dylan makes reference to this conventional understanding of recovery by saying that he can't pretend he's symptom free. Second, there is the notion of recovery as meaningful and purposeful work (lines 172 to 175 in the first data extract). Productive and meaningful activity, including employment, is included in practical tools for recovery such as the Recovery Star and the CTP approach, though this version may also have neoliberal overtones (McKeith and Burns, 2010; Welsh Government, 2015). This recovery version is found in other participants' talk $(n=4)$ as indicated by the concept 'Productive and occupying work' in the concept map shown in Figure 7. Third, there is the version of recovery grounded in social inclusion and developing relationships (Amering and Schmolke, 2009; Bonney and Stickley, 2008; McCranie, 2010; Repper and Perkins, 2003). In the first extract, Dylan states that "connecting with people, engaging with people" (lines 170 to 171) is a crucial part of self-management in recovery. The latter two recovery versions are found in a fuller form in my analysis of subsequent participants' talk in this thesis.

Summing up, no fewer than six versions of recovery can therefore be identified in Dylan's talk. This finding produced by my analysis provides some support for the viewpoint in section 2.6.2 in the literature review that recovery is a polyvalent concept. Earlier on in the analysis provided in this section, I highlighted that Dylan's describing recovery as "a bit ambiguous" (line 161) was a reference to the contrast between the conventional understanding of recovery-as-cure, and the mental health understanding of recovery as a perpetual, lifelong journey of daily, vigilant self-management. I would add that the evidence provided by analysis of Dylan's talk indicates that the phenomenon of competing versions of recovery is much more widespread than this single instance of ambivalence about the meaning of recovery. Analysis of Dylan's talk has shown his awareness of alternative explanations of recovery and perhaps also his own uncertainty given the shifting sands of the concept itself. The ambivalence Dylan expresses about the nature of recovery may also refer to the multiplicity of constructions of recovery versions occurring alongside one another in his talk. As has already been seen, and will be 
supported further by subsequent analysis in this chapter, these recovery versions may be complementary or they may be competing.

It is worth considering the dual nature of Dylan's position and identity as service user and third sector worker as a possible explanation for the multiple recovery versions constructed in his talk. As I described in section 4.3.2.1, the perspective of discursive psychology on identity is that it is constituted by the various constructions which agents may be responsible for in the context of different roles, functions and relationships (Antaki and Widdicombe, 2008; Widdicombe, 2008). Since Dylan has two separate identities or roles as both service user and third sector worker, he is likely to construct a greater number and variety of recovery versions than someone with a more singular identity, in order to contribute to the construction of these multiple identities.

Despite this basis for polyvalent constructions of recovery in his talk, Dylan does assert a preference for one recovery version which is the self-management approach, as indicated by his opening statement (line 160). What is interesting about this extract is the tension which exists in Dylan's account. On the one hand, he attempts to define recovery as a singular concept of self-management (line 160). However, despite this commitment to one version of recovery, his talk is notable for the polyvalency of recovery versions which he constructs drawing on a variety of discursive sources associated with different understandings of recovery. This mixing of discourses (Bakhtin, 1981; Bakhtin, 2010; Fairclough, 2003), is indicative of the polyvalent and unstable nature of the recovery concept, yielding multiple versions in his talk. It is also indicative of the way Dylan draws upon multiple conceptualisations of recovery to craft a sophisticated narrative that shows awareness of competing versions, exhibiting varying degrees of appropriation and colonisation. However, he is primarily concerned in using his own advocacy of an emancipatory self-management version to contrast with more colonised versions based on the medical model or neoliberalism. In the next account and accompanying analysis, I examine an instance of neoliberalist colonisation of recovery in the form of recovery-as-work, which provides further support for my argument that there are polyvalent versions of recovery being constructed in the data set. 


\subsection{3 "Recovery for me would be where I feel well enough to work productively": Competing versions of recovery inhibiting experienced continuity}

In this section, I present analysis of a data extract from a research interview with another participant, Alun. Alun is a service user who was recruited and interviewed during the first phase of data collection. Unlike Dylan therefore, since he is in receipt of services, he occupies the space at the interface of primary and secondary healthcare. That Alun is a service user in terms of my specific research focus might imply his experience would embody a less colonised version of recovery grounded in the lived experience of people with mental illness (Deegan, 1998; Edgely, 2012; Repper and Perkins, 2003). However, I argue in analysis which follows that a key recovery version he constructs, recovery-as-work, is influenced by a degree of colonisation from external, neoliberalist forces. One of the consequences of this is that this recovery version competes with other recovery constructions in his talk in such a way that implies an impediment and discontinuity related to his care.

Alun had been discharged from secondary mental health services within the past three years at the time of interview. In the interview, I raised the subject of recovery in mental health by asking the appropriate question from my interview schedule. In the following data extract, Alun seems immediately to have a good grasp of recovery in the sense given in mental healthcare, as opposed to the conventional understanding of recovery-as-cure. Alun's version of recovery is based on adopting productive, meaningful and occupying work and is shared by others in the data set $(n=3)$, as represented by the concept 'Productive and occupying work' in Figure 7. The following data extract of talk occurs at the point in which Alun expounds this version of recovery-as-work.

520. Alun: Recovery for me would be where

521. I feel well enough to work productively without stress and

522. to be happy in my work and sufficiently well to work in a

523. job where I was I was making enough money - I used to

524. be a journalist and, although it's not the best job in the

525. world, it provided enough of an income to survive quite

526. well. I'm not at that stage where I could do that now. I

527. don't want to go back into journalism but I do feel - I

528. wish I was well enough to work - I'm not, therefore I'm

529. on benefits, therefore I don't feel as great as I could. And

530. therefore my mental health isn't as good as it could be. 
531. So, it's kind of a vicious circle - it's chicken or egg - do

532. you know what I mean?

533. Researcher: Yes I do know what you mean .

534. Alun: And that's an important part of

535. recovery, having that vocational focus.

536. Researcher: Yes.

537. Alun: But that's not the only thing I feel

538. about health. I feel that that's uhm that's the important

539. one. But what I do feel about health is when I'm happy,

540. health uhm (.3) Having no negativity. Able to have fun and

541. relationships and enjoy life before I die. ((Laughs)) Do you

542. know what I mean?

543. Researcher: Yes I do.

544. Alun: You've got to try and make the

545. most of it while I'm here. And when you're unwell or

546. feeling unwell (.)2 you can't enjoy so (.2) it's a pity so (.2) I

547. would like to be able to maximise my opportunities to

548. have a good life, and at the moment I feel because I

549. haven't got a CPN and because I haven't got the

550. continuity of care I'm not able to do that. Off my own bat -

551. I'm not sufficiently independent with my health at the

552. moment to be able to do that. Even though secondary

553. mental health keep saying to me or have said to me

554. before - well uhm we're discharging you (I think on that

555. now a minute). You know, I'm not right. How can you

556. discharge me? But it falls on deaf ears. So that's my

557. frustration.

[Alun, Service User, Phase 1, Participant 1, lines 520-557]

Alun initiates his talk on recovery by describing it from a personal point of view: recovery is what it is "for me" (line 520). Recovery for Alun is being able to "work productively" (line 521 ) or "having that vocational focus" (line 535), though he attaches a number of caveats to this notion. This kind of work-as-recovery should be "without stress" (line 521), he would want to be "happy" (line 522) in this work and "sufficiently well" (line 522) to be making "enough money" from this work supporting recovery (line 523). He then develops further this last attribute about income by recounting his past experience when he was sufficiently well to have worked as a journalist (lines 523 to 526). Alun introduces a brief narrative account of having previously been a member of this profession, and indicates that this line of work "provided enough of an income to survive quite well" (lines 525 to 526). The phrase 'survive quite well' appears to be a form of speech referring to a past state of affairs where Alun was doing well financially. This prosperous state of being is hedged by the contrasting use of the word 'survive', presumably to affect a kind of modest reticence about this past state of prosperity. 
By employing this narrative account (Riessman, 1993; Riessman, 2008), such a state of prosperity is put forward as an important aspect of the recovery-as-work version Alun is constructing, where he would be making "enough" money (line 523).

Crucially, Alun constructs his account in such a way that recovery-as-work would involve a job which would be capable of delivering a level of prosperity that would support his recovery. Currently however, Alun states that he is not well enough to engage in this kind of work which would produce significant earnings (line 528), and consequently he is on benefits (lines 528 to 529). He then says that his current status as unemployed and on benefits is the key contributor to him not being as well (lines 529 to 530), which in turn acts as an impediment to him finding work that would support his recovery and lead to him being well. This then is a kind of vicious circle situation that Alun finds himself in (line 531), a state of affairs which Alun refers to by the use of the "chicken or egg" (line 531) metaphor. The use of this metaphor has a performative effect as well as a descriptive one, reinforcing the construction in his talk that he is in a vicious circle.

Alun therefore constructs two versions of recovery in his talk. The first is based on productive work, as outlined above. The second is based on wellbeing and quality of life (Bowling, 2005; Edgley et al., 2012; Mind, 2008). Health, in the sense of recovery-based mental health (line 538), entails a number of things according to Alun: being "happy" (line 539), "having no negativity" (line 540), being able to have fun and enjoy life (line 541) and being able to enjoy "relationships" (line 541). These ideas are then summarised with the phrase about being able to "maximise my opportunities to have a good life" (lines 547 to 548). Alun's notion of living a good life can be related to quality of life theory on health and wellbeing (Bowling, 2005; Connell et al., 2012). One of the foundations for this understanding of healthcare is the definition of health provided by the World Health Organisation which focuses on positive health rather than on an absence of illness. According to this definition, healthiness is "a state of complete physical, mental and social well-being and not merely the absence of disease and infirmity" (WHO, 1948, p.100). This definition, emphasising wellness and not merely an absence of illness, has been developed into the modern quality of life movement focusing on positive health, functional ability and wellbeing (Bowling, 2005; Crawford et al., 2011; Sullivan, 2003). Wellness and quality life is the focus of certain versions of recovery in the 
literature (Edgley et al., 2012; Mind, 2008; NIMHE, 2005), a perspective which is reflected in this part of Alun's talk.

To an extent, these two versions of recovery in Alun's talk are related, since a degree of wellness is a prerequisite for Alun to be able to do the kind of work which contributes to his recovery (line 522). At the same time, they are competing versions since they potentially impede and negate one another. On the one hand, Alun states he needs to be "sufficiently well" (line 522) to be able to do work to support his recovery. On the other hand, being able to work is a precondition for Alun to be well. Alun's talk conveys a double-bind situation (Bateson et al., 1956) due to his construction of two competing versions of recovery which are set at odds with one another. There is a kind of circular argument in Alun's talk which generates a state of affairs of self-defeating circumstances for himself. The implication is therefore that Alun's care progression has been impeded and stuck in a state of impasse because of the competing nature of the two recovery versions in his talk. This constitutes a state of discontinuity of care for Alun, especially in the dimension of experienced care continuity which would entail an experience of care and treatment as a continuous and seamless whole (Poremski et al., 2016; Rose et al., 2009; Weaver et al., 2017). This is certainly not the case given the state of impasse which Alun expresses himself as being in. Because of the oppositionality which occurs between competing versions of recovery, Alun cannot experience "care as continuous, connected and coordinated such that no detrimental gaps in provision have occurred" (Weaver et al., 207, p.435). Additionally, this construction has rhetorical implications since Alun's argument is constructed in such a way as to make it almost impervious to challenge. There is nothing that can be said to disrupt this version of affairs, at least superficially. However, Alun locates responsibility for the "vicious circle" (line 531) scenario not with an impediment due to competing recovery versions in his talk but with secondary services and the difficulty he has in accessing them (lines 552 to 557). This ties in with his overall construction of his account in this interview as a complaint.

It is interesting to ponder the nature of the impasse or discontinuity of care which is produced in Alun's talk by construction of competing versions of recovery. Alun's description of selfdefeating circumstances can be seen as a product of Alun's inability to step out of a state of dependency on services and into a self-managed form of recovery. Related to this, recovery in 
one sense can be interpreted as challenging the traditional Parsonian 'sick role' (Edgley et al., 2012; Parsons, 1951b; Pilgrim and McCranie, 2013), according to which, a person who is unwell and therefore no longer able to be a productive member of society, may have their deviance sanctioned by temporarily adopting the sick role allowing their normal social function to be suspended. Recovery offers an alternative to the sick role through an emphasis on building self-empowerment and illness management skills whilst sickness continues in a way which is not administered by the clinician, and where there would not therefore be dependence upon services (Pilgrim and McCranie, 2013). This may include finding productive and meaningful work and the opportunity to move beyond the constraints of the 'sick role' whilst continuing to have mental illness to some degree (Edgley et al., 2012). Alun's construction of two competing versions of recovery creates an impasse in his care which embodies in his talk his thraldom to the Parsonian sick role where he remains dependent upon services, unable to step out into a new level of independence and self-sufficiency offered by many versions of recovery. This may not be Alun's fault since he may not have been properly informed about or supported in his potential to step into a level of recovery-based self-sufficiency. In this sense, Alun's account may represent the conflicted nature of contemporary mental health care in which he is expected to move towards a recovery he may not feel able or adequately prepared for. Alun is a product of the system of care which has traditionally fostered dependence and removed or relegated decision making by patients in favour of them being made on their behalf by clinicians and workers (Braslow, 2013). The recent move towards recovery-based services emphasising self-sufficiency does not seem to have been communicated successfully to Alun, and he is out of step with this development. This incongruency may have been a factor behind the confusion inherent in construction of competing and contradictory versions of recovery by Alun, leading to a profound discontinuity in his care, inherent in his state of impasse.

Additionally, it may be said that Alun's version of work-as-recovery is a highly colonised construction, influenced by the neoliberalist agenda supporting a return to work and reduced dependency on services (Becker et al., 2010; Braslow, 2014; Mind, 2008; Morrow, 2013). Potentially this is due to his having been socialised into a system that promotes a narrow interpretation of recovery as leaving a state of 'dependency' and returning to work (Morrow, 2013; Ramanuj et al., 2015). I have argued that this is represented to a certain extent by the Mental Health (Wales) Measure 2010. The presence of such a colonised recovery version in 
Alun's talk may partly explain its oppositionality to a less colonised recovery version based on wellness. As I proposed in the introduction to this chapter, different instances and degrees of colonisation are an important factor behind competing versions. In this case, a colonised version of work-as-recovery has led to its construction which stands in contrast and opposition to a less colonised recovery version based on wellness. Instances of colonisation of recovery such as this therefore afford a degree of explanation for why multiple, competing versions of recovery may be generated. This has the effect in Alun's talk of engendering an impasse or discontinuity in his care so that he remains 'stuck' at a certain point, and unable to progress in his recovery or care. Such discontinuities of care may be more generally related to service complexity and fragmentation (Gilburt et al., 2014; Weaver et al., 2017). One element of service fragmentation is the emergence of a great role for the voluntary and third sector as secondary mental health care services retreat from more extensive mental healthcare provision. I focus on this in the next section.

\subsection{Third sector recovery care and support}

I now move onto a second concept map relevant to the theme 'Competing recovery versions' and related talk under the category 'Third sector care and support'. From this point onwards, in this chapter I construct the theme 'Competing versions of recovery' entirely from talk of professional participants who are not service users. This reflects the greater amount of talk on recovery amongst professionals recruited in the second phase of data collection $(n=15)$ compared to service users recruited in the first phase of data collection $(n=10)$. This was not due to a greater amount of prompting and probing about recovery with second phase interviews since this was identical in both interview schedules (see Appendices 1 and 2). One explanation might be that recovery at the conceptual level is perhaps easier for professionals to ponder but for people living with long-term conditions it can appear as something too abstract and not immediately relevant to their day-to-day experiences. I also saw this as indicative that in the data set, the concept of recovery is increasingly not primarily rooted in the lived experience of service users, but has rather been colonised to varying degrees by the interests and agendas of workers and professionals, both in services and in the third sector. Such processes of colonisation I would argue contributes to recovery increasingly being seen as something abstract and not relevant to the immediate lived experience of service users. 
The following concept map shows the major concepts analysed from participants' talk in relation to the category 'Third sector care and support'. It can be seen from this figure that a great deal of the talk on care and support is related to recovery, given the key concept, “'Recovery' approaches'. Other concepts under the category 'Third sector care and support' are more evaluative such as 'Positive experiences of third sector support' and 'Resource issues for third sector':

\section{Figure 8: Third sector care and support}

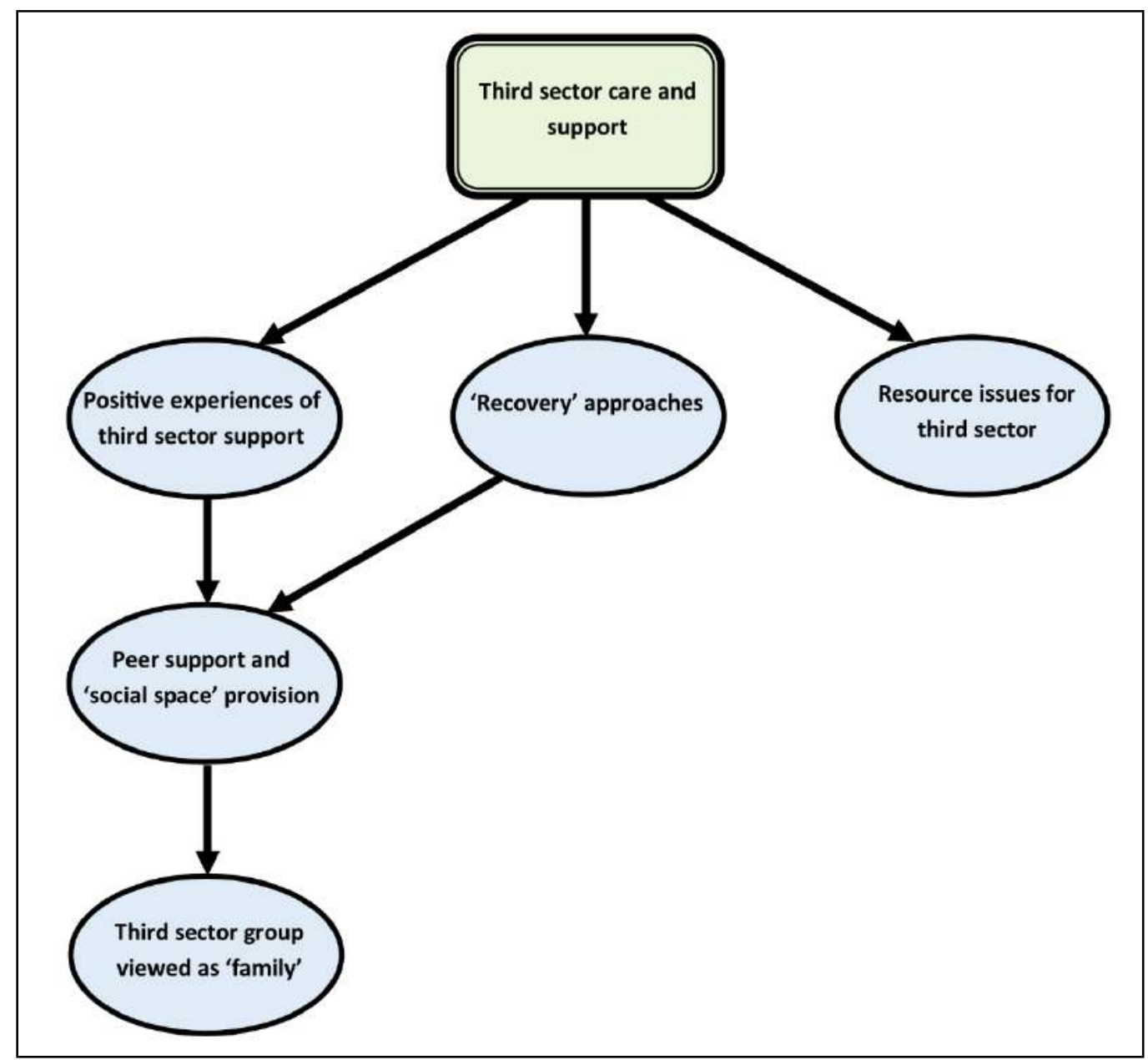

This concept map also shows that a particular version of recovery emphasising social connectedness and peer support was predominant amongst third sector participants' talk (concepts 'Peer support and 'social space' provision' and 'Third sector group viewed as 'family'). This is a version of recovery, already touched upon by Dylan (lines 170 to 171), emphasising social inclusion and relationships (Amering and Schmolke, 2009; Bonney and 
Stickley, 2008; McCranie, 2010; Repper and Perkins, 2003). Social inclusion and the development of relationships is an important dimension of recovery approaches amongst associated theories (Davidson, 2005; Edgley et al., 2012; Gilburt, 2013; Repper and Perkins, 2003; Tew et al., 2011). Data from third sector worker participants collated in this concept map $(n=8)$ constituted talk about the value of the third sector day centre making available peer support and also providing a social space for attendees (concept 'Peer support and 'social space' provision'). Some participants $(n=3)$ used the metaphor of family to characterise their organisation's provision of social support and inclusion. The following data extract from a Phase 2 participant, Tony, is one such instance of the use of this metaphor within talk that positions social inclusion-based recovery as being important within third sector day centres.

\subsection{1 "It's like children, both parents have got to be stable, and that's the core thing": Recovery versions in third sector care}

Tony is a manager of a third sector day centre. This centre is also a drop-in centre where people with SMI may come for support, care and company. Volunteers within the organisation also provide one-to-one counseling, art therapy and psychotherapeutic interventions such as CBT (lines 83 to 84 ). In this context, Tony launches a complaint within his interview based on a critique of services, specifically primary care. He initiates this critique with a narrative account in the following data extract berating the limited and one-dimensional nature of mental health care through the GP, which consists only of a prescription of diazepam:

35. Tony: A long time ago, way before I got involved

36. in working in mental health services I had lots of drug

37. issues. Not a very nice childhood, always around drugs

38. in my childhood. I was using drugs from around the age

39. of eleven. And so when I was progressing onto harder

40. things we used to try and get prescription drugs - things

41. like diazepam, temazepam ${ }^{12}$ and things like that. When I

42. was about fifteen, sixteen years old we would try to do

43. that and we'd go to GPs you know and kind of - and I

44. was a very good liar and they wouldn't give me anything.

45. And I would sort of present in certain ways to get that

46. medication. And I said a lot of things and the right things

12 Diazepam and Temazepam are types of a class of drugs known as Benzodiazepines. Diazepam is more commonly known as Valium. 
47. I would have thought to get that kind of mood, that

48. something to just lower, and they wouldn't ever, and I

49. wasn't messy, I presented myself - (.2) you wouldn't have

50. known. Does that make sense?

51. Researcher: Yes.

52. Tony: So that's going back, kind of, the nineties.

53. Sort of mid-nineties. I am going somewhere with this.

54. Researcher: Yes, take your time.

55. Tony: Fast forward now.

56. Researcher: Yes.

57. Tony: If someone comes here and says that

58. they're going to take their own life and they've visualised

59. how they're going to take their own life. Someone comes

60. here who says they have panic attacks when they go

61. shopping. Or someone comes in here who says, erm,

62. that they might have intrusive thoughts, obsessive

63. thoughts. They always seem to get diazepam.

64. Researcher: Okay.

65. Tony: That's it.

66. Researcher: Right.

67. Tony: And I'll see you later. And it's never been

68. like that - it's a real modern phenomenon, I'd say it's

69. about the last two years.

70. Researcher: So what was it like before then?

71. Tony: Well usually they'd be at least referred onto

72. community mental health team or referred onto some

73. sort of services or, you know, some people are pinballed

74. back down to here, so you'll get that they've already

75. gone there and the GP might have heard of us. We try

76. and do about as much outreach as possible where we go

77. to GPs. I've spoken at GP surgeries and things like that

78. and TRIAGEs like they've got at Milford. But the point I'm

79. making is they just chuck diazepam at them. That's what

80. they do. It seems to be all they do.

81. Researcher: And what are the results of that approach?

82. Tony: Not really anything. We'll provide one-to-

83. one talking, you know, one-to-one therapies or group

84. CBT therapies as much as we can do. With me, when I

85. became the manager here it was a big thing of mine to

86. have communication and continuity between the people

87. that work here and the centre itself so that you have that

88. base.

89. Researcher: By the centre do you mean the CMHT?

90. Tony: No, I mean here.

91. Researcher: Oh, between the people coming here.

92. Tony: So that there's always that stability, and

93. that's as much as I can, you know. It's like children, both

94. parents have got to be stable, and that's the core thing. 
95. So we try and do that and promote that. But if people are

96. just being given - it's always diazepam, diazepam. I don't

97. know what that's about, it's a real new thing for me, well,

98. not new - two years.

99. Researcher: So it's really just a new phenomenon over

100. the past two years?

101. Tony: I don't know why it's diazepam all the time?

[Tony, Third sector manager, Phase 2, Participant 7, lines 35-101]

Narrative accounts such as the one conveyed in this data extract may be understood as ways of selectively constructing stories or meta-narratives about the world (Riessman, 2008). A narrative is developed in this data extract by Tony when he recounts his teenage experience of finding it difficult to obtain prescription drugs from the GP for recreational use (lines 35 to 50). This is then compared to the present time, or the last "two years" (lines 69, 98 and 100) during which he expresses frustration that GPs now seem very willing to prescribe diazepam to people experiencing acute relapse in their mental health condition, and whom it might be argued would benefit from a less limited provision of care.

In keeping with the notion of discourse as a 'speech act', which I introduced in section 3.5.3, this concept may be extended to the idea that interviews are a form of performance or dramatisation (Crotty, 1998; Goffman, 1959; Harrington, 2005), which certainly has applicability to this data extract. This understanding has its origins in the work of Erving Goffman (1959), in whose theory, an analogy is made between social research and the theatre, with social actors playing the roles of director, actor and so forth (Harrington, 2005). In accordance with this perspective, I identified in my analysis of Tony's talk highly dramatic statements and exaggerations used rhetorically to promote the particular world view he is constructing. In this extract, the depiction of GP care consisting of "just giving diazepam" (lines 63, 7996 and 101) can be considered to be an exaggeration and dramatisation constructing a picture of inadequate primary mental health care provision which is overly medicalised in that there is an overreliance on (a very limited range of) medication. This can also be understood as an Extreme Case Formulation where a disagreeable state of affairs is exaggerated rhetorically in order to enhance Tony's critique (Pomerantz, 1986): it is highly unlikely that it is literally the case that all GPs in the area of West Wales always only prescribe diazepam for people with mental health problems. I therefore considered Tony's account to be 
a dramaturgical and exaggerated 'speech act' coloured by the unique perspective entailed in the cultural and social background of the participant (Atkinson et al., 2003; Coffey and Atkinson, 1996). This talk then is less about a literal representation of the actual state of affairs, and much more about a performative accomplishment, designed to provoke a response in the reader to sympathise with Tony's critique. This dramatised narrative version of events metaphorically expresses the participant's genuine frustrations with the real difficulties involved with current primary mental healthcare provision in Wales.

Tony then contrasts the shortfalls of primary mental health services with his own organisation's provision of care. He provides a favourable account of his organisation's care and support based on a claim of empathetic and personal treatment of clients. This account rests upon a version of recovery emphasising social inclusion and culminating in a family-related metaphor, employing terms relevant to this discursive domain such as "children" and "parents" (lines 93 and 94). This metaphorical construction is further enhanced by the use of additional familyrelated metaphors by Tony later on in the interview, which I present in the following data extract:

263. Tony: It's like getting your father starts displaying in a

264. certain way, your sister starts displaying a certain way.

265. You know that person inside and out, you've spent a

266. long time with them.

[Tony, Third sector manager, Phase 2, Participant 7, lines 263-266]

These metaphors, such as "father" (line 263) and "sister" (line 264), are employed to describe the therapeutic relationship built over time between third sector workers and clients, in which context a deterioration in mental health may more easily be detected.

Although Tony in this way constructs and endorses a recovery approach for his organisation based on social inclusion, this recovery version shows signs of being colonised by other approaches to mental healthcare and support. Talk on the provision of familial stability (lines 92 to 94) may be considered to be taken from discourse related to psychoanalytic attachment theory and the need for a secure-base in order to create the conditions for good psychological 
health (Bowlby, 2012; Willig, 2013). Further talk from the psychotherapeutic domain is present in Tony's talk about one-to-one counseling (lines 82 to 83) and "CBT therapies" (line 84).

Willig (2013) describes how terms may be taken from particular discourses or genres to construct an account, which is what I argue is being accomplished here in Tony's talk. Norman Fairclough's (1992; 2003) approach of critical discourse analysis is relevant here to an understanding of how discourses operate within the wider context of other discourses and society. The concept of interdiscursivity Fairclough provides describes how different discourses and genres are articulated together in a communicative event (Jørgensen and Phillips, 2002). Discursive objects or terms may be borrowed from other discourses (Willig, 2013) as happens here in Tony's talk to construct a version of recovery which is colonised by elements and ideas taken from the domain of psychotherapy.

The colonised nature of the version of recovery which Tony presents reflects a wider picture of recovery-appropriation throughout the third sector. Despite the working assumption that third sector organisations successfully embrace and apply recovery approaches in a straightforward way (Edgley et al., 2012; ORS, 2014; Pilgrim and McCranie, 2013), this view may be regarded as simplistic (Gilburt et al., 2014; Mind 2008). Third sector organisations may be involved in a variety of supportive activities not necessarily originating from within the recovery paradigm. One of these is rehabilitation which was clearly distinguished from recovery early on in the recovery movement's history (Deegan, 1988). Additionally, although there is great awareness of the idea of recovery and claims of its application within the third sector, there is wide variation of recovery activities across such services in the UK (Simpson et al., 2016b). It may be more accurate to say that 'recovery' is an appropriate label used by the third sector to incorporate and legitimise activities which may or may not originally reside within the recovery paradigm. These organisations see an alignment between their values and what recovery sets out to achieve, going on to construct their own versions and colonisations of recovery. Admittedly, this pattern of appropriation is not confined to the third sector, as is seen in the variety of recovery versions in the literature, in the form of neoliberalist recoverybased policies, and constructed in the talk of participants analysed in this study (Bonney and Stickley, 2008; Braslow, 2013; Leamy et al., 2011; Morrow, 2013; Pilgrim and McCranie, 2013). 
Tony's version of recovery within the third sector, whose colonised nature I have identified in preceding analysis, is employed as a favourable version of care to contrast with a negative portrayal of the GP's provision in the first data extract provided in this section. A favoured version of recovery is therefore being used to cast the GP provision in a negative light. This has potential implications for discontinuity of care since certain service users may benefit from a combination of care and support from the third sector and from the GP, where there is some level of collaboration and harmonisation of activities. For instance, some participant third sector workers $(n=3)$ spoke of the support they could provide by accompanying clients to appointments with their GP (see concept 'Specific accounts of third sector support for GP care' in Figure 10 in the next section). However, Tony's talk indicates oppositionality between the work of the GP and the work of his voluntary sector organisation. This is reflected in the construction of his recovery version and the perception that this is incompatible with the work of the GP who does not follow this approach, and whose approach is heavily criticised in the above dramatised account. To develop this point about oppositionality between third sector recovery and primary care, I focus in the next section upon talk related to third sector collaboration with the General Practice and the role that recovery versions have at this interface.

\subsection{Third sector collaboration with primary care}

The final section of this chapter focuses on the third category covered by analysis in this chapter related to third sector collaboration and working together with primary care. As will be seen from both conceptual and discourse analysis of talk, the issue of collaboration at this interface is affected by competing versions of recovery held by different groups and individuals in the service system. Collaboration between primary care and the third sector is now advocated at a global level (WHO, 2008), and more locally has been part of the focus of recent policy initiatives such as the 'Big Society' in the UK (Alcock, 2016; Kisby, 2010), and 'Together for Mental Health' in Wales (Welsh Government, 2012b). Political visions such as the Big Society represent an attempt to shift from 'big state' to 'big society' based on core themes such as empowering communities and promoting a culture of volunteering (Alcock, 2016; Humphries and Curry, 2011; Kisby, 2010). These political approaches in the UK and Wales purport that services alone are not sufficient to deal with the health and social care needs of society, 
including mental health (Baggott, 2016). From another perspective, these approaches can be seen as examples of neoliberalist influenced policy which recommends a minimal or "night watchman' state, low taxation married to public spending cuts in health, welfare and education, and promotion of the free-market as the governing and organising principle in society (Heywood, 2007; MacGregor, 2005; McKeown et al., 2017). However, despite promotion of this policy ethos in the UK and Wales, the agenda has been criticised for attempting to replace necessary public services with the goodwill and activities of volunteers (Kisby, 2010). By 2015 the Big Society rhetoric had been largely abandoned by the government, since it was perceived as a defunct and empty concept (Alcock, 2016). Despite this, a vision for appropriate and balanced collaboration between primary care and the voluntary sector has been retained by policy makers, including in Wales, though with acknowledgement of some of the difficulties and limitations which may apply to this type of cooperation (Fleury and Grenier, 2012; Welsh Government, 2011b; 2012b).

Although Welsh governance is less committed to Conservative Party philosophies, policies emerging from the Welsh Government arguably have a neoliberalist flavour. This can be seen with prudent healthcare principles (Aylward, 2013), and is also noticeable in policy attempting to define the landscape for the configuration of relationships between the third sector and services (Welsh Government, 2011b). In the mental health tiered system for adults for instance (Figure 9), primary care and third sector services both occupy tier one, implying they should have close proximity in the Welsh mental health system: 
Figure 9: Mental health whole system: a tiered model for adults

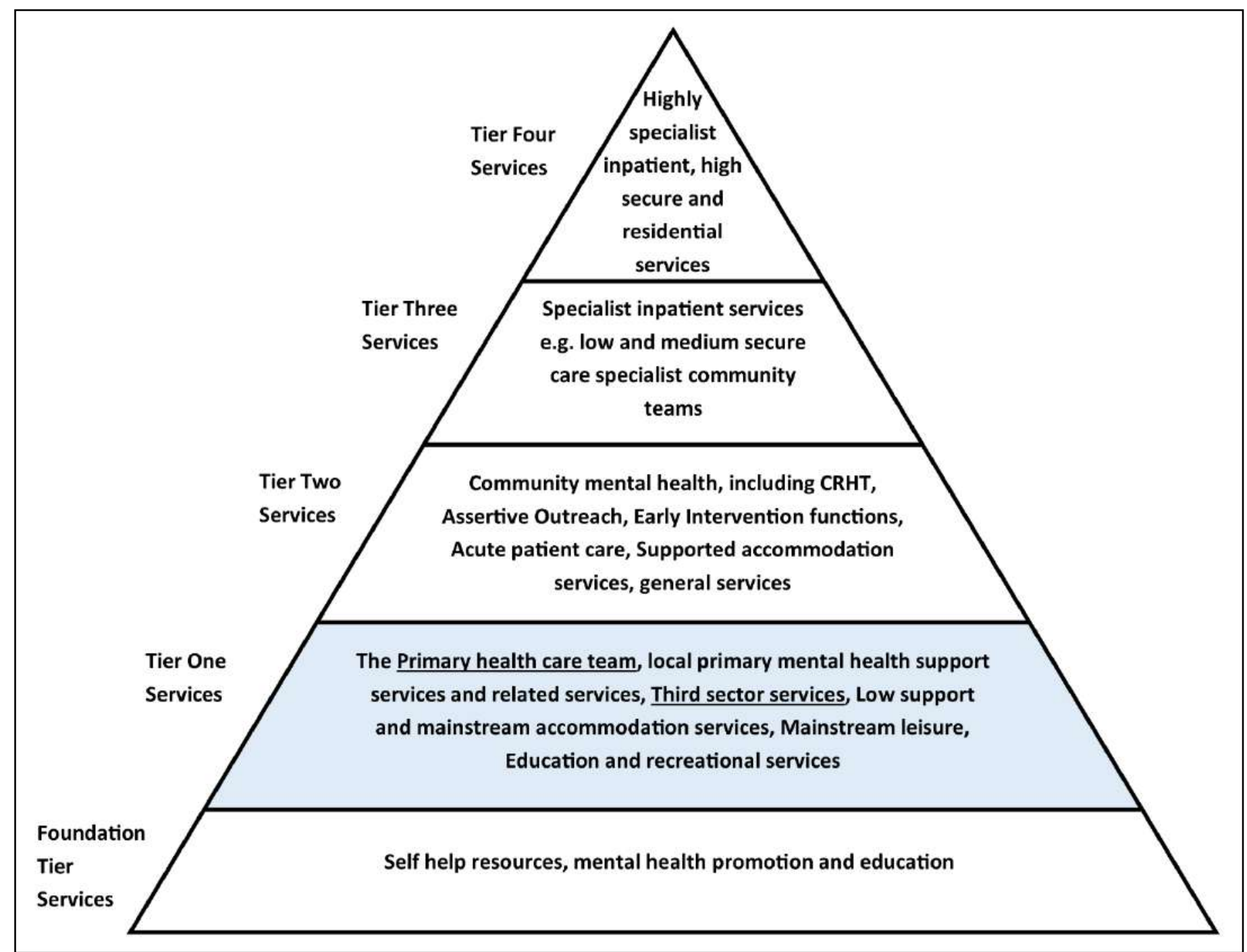

(Welsh Government, 2011b, p.28)

The culture of services in Wales is influenced by such governance seeking to encourage a major role for the third sector at the primary level, with the result that various groups recommend there should be positive engagement between third sector organisations and public services, including General Practices (Gofal, 2013; WaMH in PC, 2015).

With respect to this theoretical and policy context, I now continue my analysis developing the theme 'Competing versions of recovery' by analysing participants' talk about existing or potential working together between the third sector and primary care, related to the category 
'Third sector collaboration with primary care'. These ways of working together or not working together are predominantly framed within the rhetoric of recovery approaches or versions, as the following concept map shows (Figure 10). This partly reflects the prominent place for recovery versions appropriated by the third sector (Edgley et al., 2012; ORS, 2014; Pilgrim and McCranie, 2013). The place for a recovery approach within primary care is less developed and more controversial generally speaking (Lester and Gask, 2006; Ramanuj et al., 2015). However, in the Welsh context, the specific policy implementation of the Mental Health (Wales) Measure 2010 emphasises a self-management version of recovery carried out in the context of primary care (Welsh Government, 2010a; 2013; 2014c). Since my research focused on recovery and care continuity at the interface of primary and secondary care, the impact of this piece of legislation was relevant since it seeks to implement recovery-based services at multiple service levels including the third sector.

The following concept map collates codes referring to talk about recovery both in the third sector and in primary care. That there are competing versions of recovery within the third sector and primary care has implications for the degree to which collaboration between these sectors may be appropriate or achievable. This in turn has implications for continuities or discontinuities of care in services, particularly at the interface of the third sector and primary care. 


\section{Figure 10: Third sector collaboration with primary care}

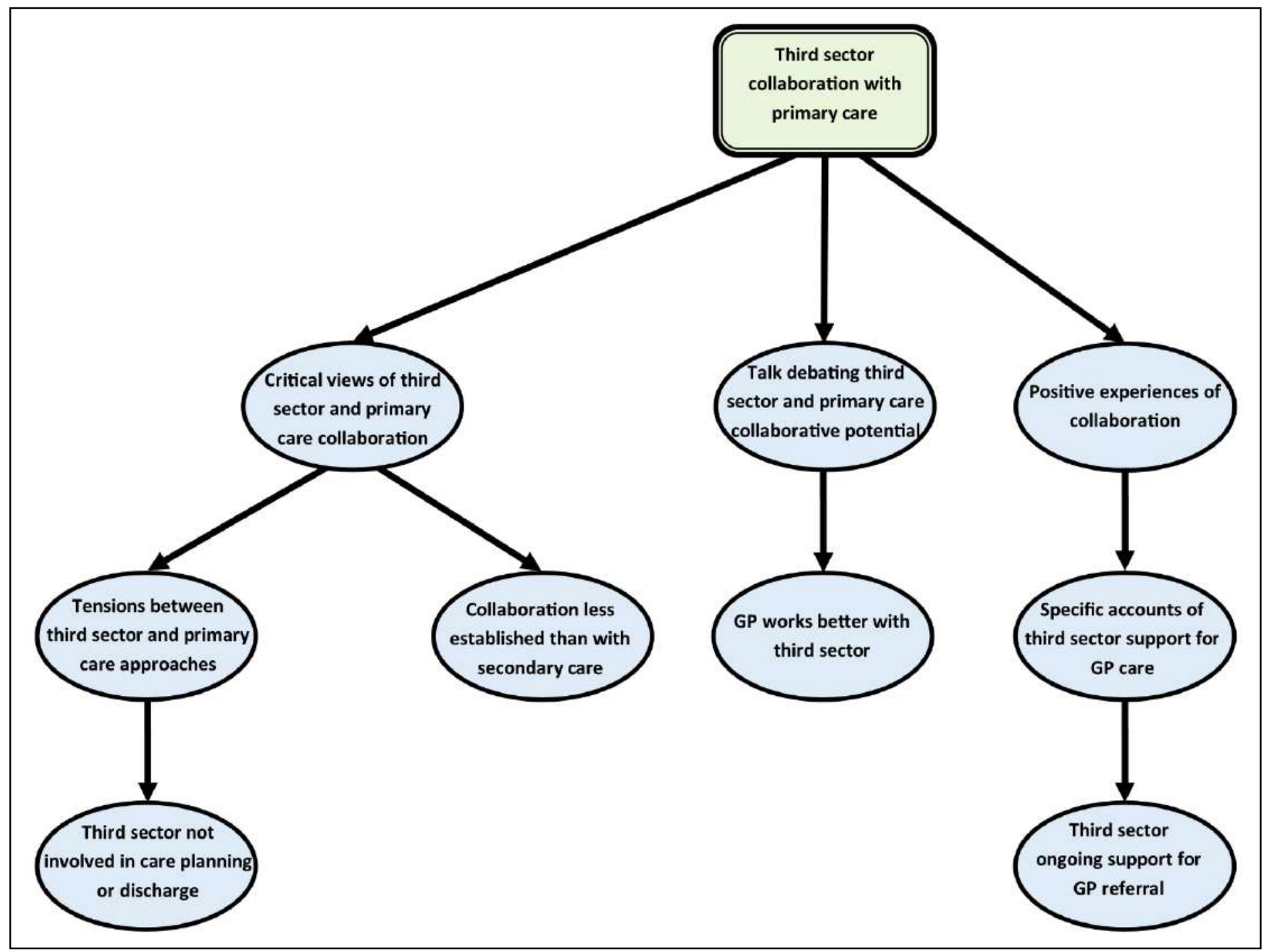

This concept map shows various aspects of third sector collaboration with primary care. A number of positive experiences of collaboration between the third sector and primary care were described in participants' talk ( $n=5$; concept 'Positive experiences of collaboration'). These are associated with 'Specific accounts of third sector support for GP care' in relation to appointment attendance $(n=4)$, where a worker would go to the appointment with the service user, even going so far as supporting clients through an entire assessment process right up to the point of referral to secondary care ( $n=3$; concept 'Third sector ongoing support for GP referral'). This would involve supporting an individual by accompanying them to an appointment, or even a series of appointments, along with ongoing ancillary support provided back at the day centre. Despite these accounts of collaboration which are talked about in 
positive terms, a large portion of the rest of participants' talk concerning collaboration is either about 'Critical views of third sector and primary care collaboration' $(n=7)$ or a debate about the need for working together to be improved $(n=6$; ' Talk debating third sector and primary care collaborative potential'). Critical talk is centred on the concept 'Tensions between third sector and primary care approaches' according to my conceptual analysis. Talk related to this concept is subjected to discourse analysis in this section. The alternate focus of critical talk according to my analysis was labelled 'Collaboration less established than with secondary care' in the concept map. This impediment to collaboration between the third sector and primary care due to it being less well-established than with secondary care could be considered a feature of the novel role primary care has in providing expanded mental health support under the Measure.

Continuing to build my argument about oppositionality between competing recovery versions and the implications this has for care continuity, especially at the interface between third sector and primary care, I now present my analysis the talk of two participants, Hannah and Robert.

\subsection{1 “I suppose that's helping people towards recovery isn't it?" Oppositionality between third sector recovery versions and primary care}

Hannah is a support worker in her twenties within a third sector organisation who I interviewed in the second round of data collection. Her talk in the research interview was generally quite negative about both secondary and primary care services, whilst also being positive and supportive of the work her third sector organisation carried out. Her discourse therefore follows similar pattern to Tony's talk where a critical account of primary care is contrasted with promotion of a positive recovery version carried out by her voluntary sector organisation. In the following data extract, Hannah presents a recovery version based on the Recovery Star (McKeith and Burns, 2010). She then contrasts this with primary care which is positioned in her account as not harmonizing with this approach. Her talk then builds negative implications about the potential for working together between her organisation and the GP to promote a person's recovery. 
300. Researcher: Yeah, okay. Final question, just focusing on

301. recovery and the recovery model. Is that something you

302. particularly focus on, recovery - (.4) do you have an

303. understanding of recovery? What's your understanding of

304. recovery?

305. Hannah: Erm, I suppose looking at the person as a

306. whole. And making sure that everything is sort of taken

307. care of because it's not one thing on its own. We do have

308. Recovery Stars, I don't know if any of the other staff have

309. mentioned it. We do have Recovery Stars. It sort of looks

310. at 12 aspects of the person's life and then scales how well

311. they're doing, or how not very well they're doing in those

312. areas and then we try and see is there anything we can do

313. about this, and then make a plan about how they could

314. move $-(.2)$ you focus on say three areas and then the dots

315. would hopefully move out as the person's score gets

316. higher. Like say social circle support and things like that. If

317. a person was completely isolated, you would try and move

318. them on a little bit in that area and then the next time you

319. might try and move them on a little bit in a different area so

320. if that's working I suppose that's helping people towards

321. recovery isn't it?

322. Researcher: Does the GP help with that at all, do they

323. provide recovery care at all?

324. Hannah: Not as far as I'm aware, no. They do often

325. make suggestions to the person like the GP said it would

326. be good if I went to the gym or if I - but it's just the usual

327. things, they would say that to anyone I would imagine. It's

328. not specific like, "Oh they told me to stop smoking and go to the

329. gym and do some exercise." Obviously those things are

330. massively beneficial, like exercise for mental health.

[Hannah, Junior third sector support worker, Phase 2, Participant 9, lines 300-330]

This data extract was prompted by my initial question about Hannah's understanding of recovery and her experience of its implementation within the third sector organisation she works for. It is interesting to note her response at first indicating her understanding of recovery. There are rhetorical indicators that her understanding is vague and imprecise. These include the use of tentative hedging phrases such as "I suppose" (lines 305 and 320) and "sort of" (line 306). Hedging is often used to manage a speaker's accountability, by softening the certitude of a claim (Wiggins, 2017), but in this case, it is more plausible to see this kind of rhetoric as indicative of an uncertainty in relation to precisely what recovery is. Such uncertainty is hardly surprising given the polyvalent nature of the concept (Pilgrim, 2008). This could also be seen 
as a response to my asking her in a research interview to articulate something that she probably feels an innate sense of rather than in a detailed and technical way. Hannah then promotes the health technology of the Recovery Star (Leamy et al., 2011; McKeith and Burns 2010) as a concrete instance of recovery in her organisation, almost as a way of attempting to clarify the uncertainty surrounding the recovery concept. The Recovery Star is an appropriate instance for this purpose since it is a detailed approach, which can be considered to occupy both the selfmanagement and holistic constructions of recovery, focusing on combined development of ten separate areas of the client's life (Managing mental health; Trust and hope; Identity and selfesteem; Responsibilities; Addictive behaviour; Relationships; Work; Social networks; Living skills; Physical health and self-care). Hannah hints at the holistic, multifaceted aspect of recovery which accords with this particular tool in the phrase "it's not one thing on its own" (line 307). She completes her account with a further rhetorical indication of her uncertainty about the recovery concept expressed through hedging about whether she has properly accounted for the concept of recovery: "I suppose that's helping people towards recovery isn't it?" (lines 320 to 321 ).

The concept of 'floating signifier' provided by LaClau and Mouffe (1985) is helpful in elucidating analysis at this point. That a signifier is 'floating' is indicative that its meaning has not yet been fixed by a particular discourse or agent and that other discourses and their agents may attempt to appropriate it within the entire order of discourse ${ }^{13}$ (Fairclough, 1995; 1998; Jørgensen and Philips, 2002). The recovery concept in general may be considered to be such a floating signifier, whose meaning is manifold, contested and appropriated by various groups with different agendas (Davidson et al., 2005; Pilgrim, 2008). Hannah at first exhibits some uncertainty regarding the meaning of the concept, which is indicative of its status as a floating signifier. She then fixes its meaning in her discourse by resorting to the concrete example of the Recovery Star (McKeith and Burns, 2010). The notion of recovery as a floating signifier may be seen to characterise discursive behaviour among participants (and agents located more widely than the sample) who construct a variety of recovery versions. These are shown through

\footnotetext{
${ }^{13}$ The 'order of discourse' is defined by Fairclough $(1995 ; 1998)$ as the configuration of all discourse types within a social field. In this thesis, I utilise this understanding to describe the entirety of discourse for the area of the social world under investigation, that is, mental health services in Wales. This is intended to avoid the confusion that might arise from the way in which discourses may be embedded within larger discourses.
} 
analysis in this chapter of various appropriated and colonised recovery versions constructed in the talk of participants. I propose that the concept of floating signifier is highly appropriate for describing the polyvalent concept of recovery (Pilgrim, 2008), whose meaning has not been fixed by a particular discourse or related agent. Instead, agents construct discourses which are engaged in a process of continual discursive struggle to fix and appropriate the meaning of floating signifiers such as the recovery concept (Bonney and Stickley, 2008; Jørgensen and Phillips, 2002; Laclau and Mouffe, 1085; Senker and Green, 2016). In this case, Hannah attempts to fix the uncertain meaning of recovery by fixing it with the meaning of the particular concrete discursive perspective offered by the Recovery Star.

Hannah then goes onto provide a critique of the GP's contribution to recovery, which contrasts with her account and fixing of the meaning of her own organisation's contribution in this area (lines 324 to 330). She offers a script formulation, or typical world, (Edwards, 1994; Gee, 2010) concerning the kind of healthcare offered by the GP, which in her view is not recovery-based (lines 322 to 324). This typical world (Gee, 2010) is of care involving "the usual things" (lines 326 to 327) that a GP would offer. This care provision lacks "obviously" (line 329), "massively beneficial" (line 330) prescriptions such as specific recommendations to exercise or stop smoking (lines 328 to 329). In many ways, Hannah actually uses the script formulation to refrain from fixing the meaning of recovery in relation to the GP, leaving it as a vague and non"specific" (line 328) notion, which is in contrast to the construction of recovery based on the Recovery Star to fix the meaning of this floating signifier in relation to her own organisation. This serves to enhance the critical account of the GP's care which contrasts with Hannah's promotion of her third sector's provision labelled as recovery. Hannah's construction of a recovery version as a credible mode of care delivery also means that she has an expectation that the GP operates in the same way. Since the GP does not, according to Hannah (lines 322 to 324), there is a perception of a lack of "help" (line 322) being provided either to support the client's progress or to collaborate with the 'legitimate' care provided by her third sector organisation, based on its constructed version of recovery.

Analysis of this account shows there is a disharmony between the third sector approach and the primary care approach, which has a lot to do with Hannah's perception of the presence of a recovery construction or the lack of it in either approach. The third sector approach involves 
a recovery version but the GP does not employ a recovery approach, according to Hannah. This is a notable perspective, given the assertion under the Mental Health (Wales) Measure 2010 that self-management in the context of enhanced primary mental health care is a key way in which recovery-based services may be implemented (Lester and Gask, 2006; Ramanuj et al., 2015; Welsh Government, 2010a; 2014c). This constructed oppositionality between her organisation's approach and that of the GP is similar to Tony's, and also implies impediments to working together between the third sector and primary care, which may entail discontinuities in the dimension of cross-boundary care continuity. Cross-boundary continuity may be defined as effective coordination of care between professionals and services involving good management of these links (Adair et al., 2005; Sweeney et al., 2016; Uijen et al., 2014; Weaver et al., 2017). Variances in approaches or expectations about approaches between primary care and the third sector may contribute to discontinuities of care across this cross-boundary dimension, which will be further exacerbated by the absence of a designated care coordinator after discharge from secondary services. This then engenders discontinuities of care in other dimensions, given the central role of cross-boundary continuity within the integrated structure of the multi-dimensional care continuity concept (Burns et al., 2009; Weaver et al., 2017).

It would appear from analysis of Hannah and Tony's accounts therefore that the potential for collaboration between primary care and the third sector is limited given construction in their talk of their own recovery versions which cannot be connected with a perceived lack of an effective recovery approach available at the primary level. However, I will argue in the next section, involving analysis of Robert's talk, a GP participant, that this disconnect, and related implications for impediments to collaboration and care discontinuities, is actually a product of competing versions of recovery, rather than the result of there not being any sort of recovery approach within primary care. This is because analysis of Robert's talk reveals a nascent version of recovery which is being constructed at the primary level. Discontinuities of care at the interface of primary care and third sector services are therefore implied by perceived incompatibilities between the competing versions of recovery constructed either within the third sector or within the primary care domains. 


\subsection{2 "I think there's a very limited understanding of that specific definition but the ethos of the definition is definitely there": Nascent versions of primary care recovery competing with third sector recovery versions}

This final section and the final part of my argument from analysis developing the theme of this chapter concerns Robert who is a male GP in his mid-fifties. His research interview was one of the longest interviews in the second phase of data collection, lasting over an hour. It was also conceptually complex, covering a number of topics and issues. Relevant to this chapter is Robert's talk on potential recovery approaches in primary care. The following data extract is taken from talk which occurs after I presented the concept of recovery to Robert by using the well-known quotation from Anthony (1993). This occurs in the context of my inquiry into the degree to which recovery approaches are adopted within primary care.

691. Robert: I think there's very limited

692. understanding of that specific definition

693. (.3) but the

694. ethos of the definition is definitely there - you know - the

695. principle underlying it , of course, we would buy into that,

696. that's part of our practice. Although I don't recognise the

697. exact words here, I recognise the principle and that's

698. something that we would follow. But I think practically

699. speaking I think we could do more. Coming back to the

700. teams idea, that would be - if we were more joined up with

701. organisations like Mind, for example,

702. (.2) that

703. would help.

704. Researcher: That's something I want to come onto as a

705. final area of focus. It's interesting when you talk about

706. third sector organisations because for somebody who's

707. been discharged from secondary care there is potentially

708. the GP and also organisations like Mind and Hafal, Links -

709. day centres, drop-in centres. There is a degree to which

710. the GP and these third sector organisations can work

711. together. Here, for example, how much working together

712. is there between the GP practice and third sector

713. organisations?

714. Robert: Not enough. I mean, we used to have a

715. third sector broker in our MDT but again that was mainly

716. focussed on older adults' issues - dementia, frailty.

717. Researcher: In adult mental health which is my focus?

718. Robert: No, we don't do enough on that front.

719. And one thing I would be quite keen on personally is - and 
720. one way to sort this issue out for us would be co-location.

721. Our surgery is too much of an island. It would be good if

722. we were co-located with some third sector organisations

723. and others.

[Robert, General Practitioner, Phase 2, Participant 13, lines 691-723]

Robert says that there is a "very limited understanding" (lines 691 to 692) of the definition of recovery provided by Anthony (1993). This is the same response I received from the two other GPs who I interviewed in my study. All three GPs were not familiar with the recovery concept in mental health, for instance in the form of this early version provided by Anthony (1993), rooted in service user experience. In a way which is characteristic of the responses I received from the other two GP participants, Robert actually shows a lot of positivity towards the idea of recovery, and can see parallel ideas that inform his current work. Robert says that the underlying "ethos of the definition is definitely there" (line 694) within primary care. In saying this, Robert alludes to an underlying tradition and philosophy within primary care, which is somewhat distinct from the medicalised approach found throughout the rest of the medical profession (Green and Thorogood, 1998; Lester et al., 2004). This primary care philosophy is based on an holistic understanding of illness rooted in its social and cultural context (Currid et al., 2012; Freeman et al., 2002). To an extent this is grounded in the care of people with chronic physical health problems who have traditionally been at the core of primary care work (Lester et al., 2004). Much of the case made for mental health care being a core activity of primary care (Currid et al., 2012; Freeman et al., 2002; WHO, 2008), is based on the idea of developing an alternative approach grounded in the strengths of primary care (Lester et al., 2004). These strengths include an ability to incorporate complexity and intuition into medical decisions and recognition of the social and familial context for illness (Currid et al., 2012; Freeman et al., 2002; Lester et al., 2004; Wilson et al., 2001). It is out of this understanding of the potential contribution that primary care can make to mental health that the Mental Health (Wales) Measure 2010 is conceived in recognising the crucial role that primary care plays in delivering effective mental health treatment (Welsh Government, 2011a).

Many of these unique strengths of primary care medicine, which may be applicable to mental healthcare, show congruence and similarity with key ideas encapsulated in various versions of recovery. This is what Robert refers to when he says that within primary care the "ethos of the 
definition [of recovery] is definitely there" (lines 694 to 695). Recovering from the effects of stigmatisation is one of the goals within recovery approaches, and is often coupled with a passage towards greater social inclusion (Anthony, 1993; Bonney and Stickley, 2008; Repper and Perkins, 2003). This is comparable with the ability of primary care to offer a low-stigma setting (Freeman et al., 2002; WHO, 2008). Another parallel between recovery and primary care is the incorporation within both approaches of a more person-centred, socially grounded approach to healthcare (Amering and Schmolke, 2009; Copeland, 2000; Currid et al., 2012; Lester et al., 2004; Repper and Perkins, 2003; SAMHSA, 2004).

In the light of theoretical comparability between the strengths of primary mental health care and recovery models, analysis of Robert's talk indicates that there is potential for development of a version of recovery grounded in primary care, which to an extent he begins to construct in the above data extract. This is also the case with the other GP participants, Alex and Tamsen, who are enthusiastic about the concept and draw their own parallels between the approach and features of primary care (conceptual analysis collated this talk under the concept 'Nascent primary care-based recovery' in Figure 7). This nascent primary care-based recovery version can be considered to be a product of a process of colonisation of the concept by some of the principles of primary care, which is expressed in Robert's talk as "buy[-ing] into" (line 695) that concept.

The understanding produced by the above analysis of a nascent recovery version colonised by primary care principles allows for a realisation of a more complex picture about the relationship between third sector activities and primary care, and the impediments to it. Rather than it being the case that discontinuities of care exist between the voluntary sector and primary care because of a lack of recovery versions within the primary sector, a more accurate picture is that discontinuities exist because of perceived incompatibilities between the competing versions of recovery held within the third sector or within primary care. Robert actually connects the difficulties associated with competing recovery versions and impediments to collaboration in his talk in this data extract (see lines 696 to 703). He makes the admission that "practically speaking I think we could do more" (lines 698 to 699), right after beginning to construct his own nascent recovery version. What more he thinks could be done is to be more "joined up with" (line 700) voluntary sector organisations like Mind. This close proximity in his talk on 
recovery and issues of collaboration with the third sector (lines 697 to 701) implies a connection between these concerns, which my analysis has indicated is due to competing versions of recovery. The way in which Robert brings together the two concepts of recovery and collaboration can be seen as a process of textual meaning-making (Jørgensen and Phillips, 2002; Laclau and Mouffe, 1985). Gee (2010) refers to this process as topic chaining whereby an ontology may be built out of concepts related in talk. The ontological structure which is implied in this data extract of Robert's talk, and in preceding analysis in this chapter, is that competing versions of recovery are related to instances of disconnect between various points of care provision in different service sectors. This has negative implications for care continuity within services, which I will investigate further in the next chapter.

\subsection{Conclusion}

In this chapter, I have shown through conceptual and discourse analysis of participants' talk that there are competing versions of recovery in their approaches to mental healthcare. These versions exhibit varying degrees of colonisation by concepts and agendas outside of the domain of recovery theories. Different competing versions of recovery are often constructed in relation to specific instances of colonisation. Dylan's talk is particularly illustrative of this since it involves no fewer than six versions of recovery. That there are competing versions of recovery in participants' talk has implications for care continuity in Welsh mental health services. With Alun, competing versions of recovery based on wellness and work-as-recovery led to an impasse in his care pathway resulting in experienced discontinuity of care. With Tony and Hannah, it appears at first that potential collaboration with the primary sector is impeded, implying care discontinuity, because of oppositionality between their recovery versions and a primary care approach which is not recovery-based. However, analysis of Robert's talk reveals that this oppositionality is better regarded as the result of competing versions of recovery, since nascent versions of recovery are being conceived through new processes of colonisation of recovery within the primary care context.

The overall picture provided by analysis of participants' talk is therefore one of fragmentation of approaches within mental health services in Wales. This chapter has shown from analysis of participants' talk, that 'Competing versions of recovery' are a key factor for this fragmentation. 
I have argued in this chapter that the capacity for varied and manifold versions of recovery to be constructed is due to the polyvalent and colonised nature of the recovery concept itself, which may best be regarded as a 'floating signifier' whose meaning and application is not fixed (Laclau and Mouffe, 1985). The challenge therefore for service development in Wales under the Measure is how the diverse character of mental health recovery approaches can be encompassed within a coherent strategy that promotes continuity of care and recovery, and does not escalate service fragmentation and complexity.

In the next chapter, I focus my analysis on experiences of care continuity and discontinuity described in participants' talk. This enables me to examine care continuity as the key element in provision of complex, recovery-focused healthcare service systems in Wales. 


\section{Chapter 6: Experienced discontinuities within primary mental healthcare}

\subsection{Introduction}

In this chapter, I introduce conceptual and discourse analysis supporting my argument for the theme 'Experienced discontinuities within primary mental healthcare'. I focus my analysis on participants' talk about healthcare experiences as they continue in primary care after discharge from secondary services. The main finding and theme I generate through this analysis is that there is a concentration of care discontinuities for people with SMI who have been discharged to primary care. Conceptual analysis presented in this chapter initially indicates this concentration, since primary care was primarily the service sector talked about by participants where I formed categories and concepts labelled as care continuities and care discontinuities (see Figures 11 and 12 in this chapter). Subsequent discourse analysis builds on this to form the theme of this chapter. Care discontinuities are also found at the service boundary between secondary and primary care as participants transit to the primary level and attempt to selfmanage their condition under the care of the GP, indicating that there is a 'gap' between the primary and secondary sectors which some individuals may fall into.

I constructed this theme and analysis in the context of the appropriate theoretical framework of care continuity (Burns et al., 2009; Freeman et al., 2002; Weaver et al., 2017), which I introduced in Chapter two. Continuity of care and the related concept of care coordination are crucial to conceptualising and appraising modern mental healthcare systems (Schultz and McDonald, 2014; Sweeney et al., 2012; Weaver et al., 2017). Additionally, the view of care continuity as a multi-dimensional concept is now predominant in contemporary theory on the nature of this healthcare understanding (Bachrach, 1981; Freeman et al., 2002; Puntis et al., 2014; Weaver et al., 2017). I have therefore employed this multi-dimensional conceptual framework for care continuity as a context for analysis intermittently at various points throughout the previous two findings chapters. In this chapter however, I employ care continuity as the primary theoretical context for analysis and appraisal of participants' experiences in talk of the transition to primary care after discharge from secondary services. 


\subsection{Experiences of practices promoting continuities of primary care}

The theme 'Experienced discontinuities within primary mental healthcare' sits across the three categories of talk from which it is generated, which are 'Experiences of practices promoting continuities of primary care', 'Experiences of practices promoting discontinuities of primary care' and 'Self-management recovery within primary care'. I provide in this chapter thematic discourse analysis ranging across the span of these categories.

The first of these categories is covered in this section wherein I provide discourse analysis of relevant talk. The category 'Experiences of practices promoting continuities of primary care' consists of concepts and talk related to experiences of activities and practices promoting continuity within primary care for people with SMI in Wales. A greater amount of talk and concepts from my analysis were related to experiences of discontinuities of care, as will be seen in section 6.3 and Figure 12. Despite this, the presence of positive accounts of activities and practices promoting continuity of care could be identified in the data set. I present analysis of talk about continuities of care first before providing analysis of talk about discontinuities of care, in order to create a balanced account within this chapter. Figure 11 shows the concept map constructed by conceptual analysis of talk under the category, 'Experiences of practices promoting continuities of primary care'.

Figure 11: Experiences of practices promoting continuities of primary care

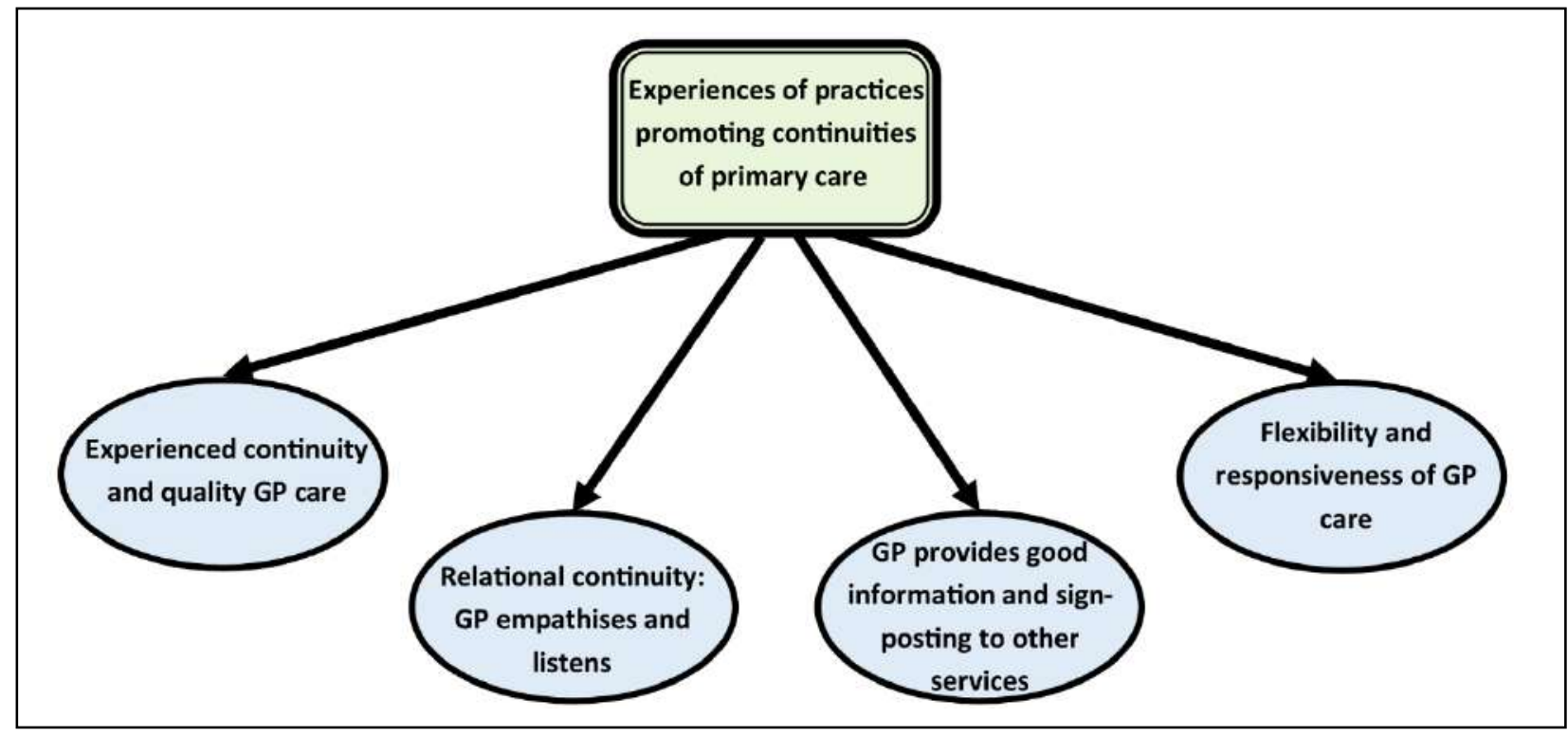


Participants' talk $(n=7)$ about experiences of practices promoting continuity of care was at first consolidated into the concept 'Experienced continuity and quality GP care'. This concept was a realisation in analysis of my data of the experienced care continuity dimension within the multi-dimensional continuity framework (see section 2.7.3). Experienced care continuity may be considered to be the overarching dimension of care continuity since the patient's perception of continuous, connected and coordinated care is paramount (Freeman et al., 2002; Heaton et al., 2012; Weaver et al., 2017). I also related talk collated under this concept to the experience of primary mental healthcare being of a good quality. Continuity of care and quality of care are closely related in scholarly discussions about the two concepts (Bachrach, 1993; Sweeney, 2016). Having first determined that care continuity is a 'fuzzy concept' (Lakoff, 1973), Sweeney and associates (2016) comment that the "haziest boundary is that between care continuity and quality of care" (p.11). The implication here is that although the concepts are not identical, they are very closely related, so that it can be difficult to distinguish one from the other. Additionally, since care continuity is a cornerstone of modern mental healthcare (Schultz and McDonald, 2014; Sweeney et al., 2012; Weaver et al., 2017), it is the key factor in determining the overall quality of care provision. Therefore, there can be considered to be a close relationship between the overall experience of care continuity and the quality of care. For this reason, my conceptual analysis of talk combined these two notions into the concept 'Experienced continuity and quality GP care'.

In addition to this, participants $(n=9)$ spoke about the GP being empathetic and listening to them in relation to their mental health issues and difficulties which can be considered to be instances of relational continuity (concept labelled 'Relational continuity: GP empathises and listens'). All of these participants were recruited at the first phase of data collection, providing first-hand accounts of their positive experiences of empathy and willingness to listen from practitioners. My analysis leads me to argue that this talk is associated with the dimension of relational care continuity which is defined as the establishment of a therapeutic relationship between one or more professionals and the service user (Belling et al., 2011; Burns et al., 2009; Weaver et al., 2017).

Participants $(n=6)$ also talked about good information and signposting from GPs towards services outside primary care ('GP provides good information and signposting to other 
services'). In all of these instances, this talk was about signposting to third sector services and day centres. Primarily, this talk occurred amongst service users recruited in the first phase of data collection ( $n=5$ ), who conveyed that they had been signposted by their GP towards the third sector setting in which they were being interviewed. My analysis led me to regard this as a practice promoting continuity of care within the dimension cross-boundary continuity, which refers to good management of links between services, professionals and components of care (Adair et al., 2005; Uijen et al., 2014; Ware et al., 2003).

Finally, I placed talk and related codes about the availability of choice of a GP within the practice under the concept 'Flexibility and responsiveness of GP care'. This concept was associated with the dimension 'Flexible and responsive continuity' (Weaver et al., 2017). Participants talked about having the option of choosing a preferred GP out of the entire group of individuals employed within the practice. This afforded a degree of flexibility and responsiveness of the primary service system to the service users' changing needs, requirements and life circumstances (Durbin et al., 2004; Freeman et al., 2002; Joyce et al., 2010).

Having described the broad conceptual analysis of participants' talk about promotion of care continuities within primary care, I now examine this and related concepts more closely through discourse analysis of participant talk.

\subsection{1 "The only doctor I now see is my GP, and he's been very good": Ostensive continuities masking discontinuities of care}

Matthew is a service user participant recruited in the first phase of data collection who provided an ostensively positive account of GP care and continuity. In the following data extract he describes that the GP has "been very good" (line 75) with regard to the care he has provided. However, in the analysis of his talk which follows, I argue that a number of discontinuities of care can be identified in his account, specifically in relation to the mental health aspect of the GP's care provision. I also argue that this is a promotional account in the sense described by Fairclough (2003), which has the discursive function of seeking to promote the GP and his care provision. I argue that this promotional account has the effect of providing ostensive 
descriptions of care continuities, which are made explicit by Matthew, whilst at the same time masking discontinuities which can be elucidated by detailed discourse analysis.

In the first part of his research interview, Matthew outlines his mental health condition, relating that he had developed epilepsy whilst he was at university. This condition has resulted in the development of some cognitive issues such as periodic confusion and difficulties with memory and concentration. Associated with this, Matthew has experienced intermittent psychotic episodes leading to past involvement with secondary, specialised services, including hospitalisation. More recently however, Matthew has been discharged to the care of the GP, thus fitting the inclusion criteria for Phase 1 recruitment. Matthew was keen throughout his interview to promote a positive account of his GP and experiences of primary care. The following data extract is a key ${ }^{14}$ passage in his interview related to this promotional account:

74. Matthew: The only doctor I now see is my GP, and he's

75. been very good, because he's helped, he's always

76. supported me with my claims for benefit.

77. Researcher: Yes, yes. So, the GP gives you

78. support for your benefits.

79. Matthew: Yes, yes.

80. Researcher: What other kind of help does he, is

81. it a he, the GP?

82. Matthew: Yes, it is, yes.

83. Researcher: What other help does he give you?

84. Matthew: Well, we have medical reviews every year. I

85. think they used to be every six months, but I see him now

86. every year. Or every ten months, I think it is, and, well, he

87. knows me very well now, because I've been under his

88. care. He knows I get anxious about money, mostly, and

89. he's always said he would back me up, because he knows

90. I have epilepsy, as well.

91. Researcher: Does he back you up?

92. Matthew: Oh yes, he does, yes. He's always, sort of,

93. backed up my claim. Whenever there's been a form to fill, (.3)

94. sorry, (.2) I've got a bit of a stammer, a form to fill out, he's

\footnotetext{
${ }^{14}$ As described in section 3.6.3, concept maps were used as a guide to navigate the data set and identify passages of talk appropriate for discourse analysis, which were relevant to the theme or argument being developed. This passage is 'key' because of its conceptual relevance to the argument being developed here: the passage is the clearest section in Matthew's talk expressing his promotional account of the GP, and also contains a concentration of codes $(n=9)$ collated under concepts of the category 'Experiences of practices promoting continuities of primary care'.
} 
95. always, because I get confused about the process

96. sometimes. He's always, I think on the last form, it was a

97. PIP form. ${ }^{15}$

98. Researcher: Was it? Did he do it for you, or did

99. he just sit by you and do it with you?

100. Matthew: No, no, it was me and my mum tend to fill the

101. forms out, or the $\mathrm{CAB}^{16}$ will fill the forms out, and, but I think

102. the DWP ${ }^{17}$ will contact the GP.

103. Researcher: Oh, I see, yes.

104. Matthew: To verify what I have is what I have, you know?

105. Researcher: Yes. Yes, of course, and he always

106. backs you up.

107. Matthew: He always backs me up.

108. Researcher: Provides a letter, or the necessary

109. phone call, that kind of thing.

110. Matthew: Yes. Yes, that's right, yes.

111. Researcher: So, do you feel he's supportive of

112. you?

113. Matthew: Oh yes.

114. Researcher: Yes. Yes, and how often do you see

115. him?

116. Matthew: Well, aside from, I mean, I don't often see him

117. much now. Only for medical reviews, unless there's

118. something else wrong. Every year.

119. Researcher: Okay, so you see him, yes, so you

120. see him, is it first and foremost for the physical condition of

121. the epilepsy?

122. Matthew: Yes, unless, because he's busy, I often leave a

123. note. When I've had a fit, I leave a note with the staff,

124. because there isn't an awful lot he can do. He did, I had a

125. fit, I remember, I mean, I hadn't had a fit for a while, and

126. they lowered my medication, and I happened to be on the

127. bicycle and I had a fit then, and they started to increase the

128. dose again. I'm on a very high dose now, and I wouldn't

129. say it's $100 \%$ under control, but it's largely under control,

130. the epilepsy.

[Matthew, Service user, Phase 1, Participant 9, lines 74-130]

In this passage, Matthew provides an account of his GP as being "very good" (line 75) primarily due to the support he provides for his Personal Independence Payment (PIP) benefits claim

\footnotetext{
${ }^{15}$ Personal Independence Payment (PIP) replaced Disability Living Allowance (DLA) under new legislation from Westminster in the form of the Social Security (Personal Independence Payment) Regulations 2013.

${ }^{16}$ Citizen's Advice Bureau.

${ }^{17}$ Department of Work and Pensions.
} 
(line 97). The nature of this support is at first unclear. However, when I probe about the precise nature of this support (lines 98 to 99), Matthew clarifies the issue by saying that his mother and the Citizen's Advice Bureau fills the forms out on his behalf (lines 100 to 101). The nature of the GP's support therefore appears to be limited to providing the backing of his professional medical opinion that Matthew continues to be sufficiently unwell to qualify for his PIP, through correspondence with the Department of Work and Pensions (lines 101 to 102). The initial lack of clarity Matthew exhibits in his talk performs a discursive function. Being vague or lacking clarity about a certain issue can be a way of inoculating claims against challenge, thus making the account more robust (Wiggins, 2017). Matthew's account functions in this way, whether he is conscious of it or not, forming a promotional account which may not stand up to closer scrutiny.

The concept of a promotional account is useful in elucidating this discursive nature of Matthew's talk. Fairclough (2003) describes promotional accounts as a product of 'promotional' or 'consumer' culture in his critical discourse theory. A typical feature of a promotional account is the employment of vagueness and obliqueness to gloss over details that might otherwise be the basis for criticism (Fairclough, 2003; Wernick, 1991). Other discursive devices used in promotional accounts are also used by Matthew. He employs the superlative term "he's been very good" (lines 74 to 75 ) which supports a positive evaluation which helps construct the promotional account. Additionally, the repeated use of the word "always" (lines $75,89,92,95,96$ and 107) can be considered to be an example of re-iteration building and reinforcing Matthew's positive, promotional account of the GP (Gee, 2008; Wiggins, 2017).

However, closer scrutiny of Matthew's account reveals areas of potential limitations in care provision and care continuity related to mental healthcare. The support provided which Matthew talks about is quite one dimensional since it is essentially a rubber-stamp confirmation of Matthew's sick status. This runs contrary to the rich and multi-dimensional nature of good care continuity described in contemporary literature (Weaver et al., 2017). Matthew reports he sees the GP quite intermittently, having reduced the frequency of review appointments from six months to once a year (lines 84 to 86 , and lines 116 to 118). This might compare favourably with the frequency of appointments a psychiatrist has with a patient with SMI on their caseload, where appointments may be monthly or even yearly (RCPsych, 2018). However, it contrasts 
sharply with more regular contacts between a service user and care coordinator within the context of the CTP approach in secondary care (Hannigan et al., 2018; Welsh Government, 2015). It might be said that it would not be expected that there would be regular contact with a GP, as this is not the case with other patients in primary care. But the typical irregular contact which characterises primary care is potentially problematic for a person in the maintenance stage of serious mental illness, which is invariably a complex condition rooted in various social determinants requiring significant time and resources to tackle them properly (Fleury and Grenier, 2012; Lester et al., 2004; WaMH in PC, 2015; WHO, 2014). Additionally, if Matthew had previously been care coordinated, the transition from secondary to primary services would represent a clear instance of a discontinuity of care being introduced. It could certainly be viewed as an interruption within the dimension of longitudinal care continuity which specifies that there should be minimal gaps in treatment (Burns et al., 2009; Puntis et al., 2014). This accords with the perception in some of the literature surrounding implementation of the Mental Health (Wales) Measure 2010 of a 'gap' between primary and secondary care (Gofal, 2015; Welsh Government, 2014c). For Matthew, there are now quite lengthy gaps in his treatment, which have increased in duration (lines 84 to 86 , and lines 116 to 118). His talk therefore shows that an element of discontinuity of care has been introduced into Matthew's experience by his discharge to primary care, which is one of the key aims of recovery under the Mental Health (Wales) Measure (Welsh Government, 2010a; 2013; 2014c). This seems to indicate that continuous, recovery-oriented care has not been maintained after discharge to primary care. Despite this, Matthew's talk conveys a sense of his being fairly content with this state of affairs, supporting my argument in this section that this account conveys both aspects of care continuities and care discontinuities in his treatment provision.

Another issue is that treatment of Matthew's physical condition of epilepsy is prioritised in this account over mental healthcare provision (lines 122 to 124). In Matthew's case, the GPs treatment of a mental health condition appears to be secondary to treatment of the physical health condition of epilepsy (lines 124 to 130). Matthew does not talk in substantive terms about the nature of any mental healthcare provision directly given by the GP in the data extract, or at any point in his interview. It may be objected that Matthew himself does not appear to prioritise his mental condition in his account, being more concerned with the support the GP provides for his benefits. However, given that Matthew has a serious mental health condition, 
it should be expected that there should be at least some clinical focus on this in treatment provided by the GP, and this is not present in Matthew's account or the entirety of his interview. More specifically, the care-giver should at least attempt to orientate Matthew towards an interest and care in self-managing his mental health condition, given the impetus towards recovery-based self-management within the context of primary care, encouraged by the Mental Health (Wales) Measure 2010. I argue that this interest, if it existed, would have been expressed at some point in Matthew's interview, in which the focus was upon mental healthcare and treatment (see the interview schedule in Appendix 1 for Phase 1, service user participants). The above data extract and the interview in general is therefore striking in terms of its lack of focus upon specific issues of mental healthcare provision for a person with SMI.

These findings could be seen to accord with a key concern levelled at the idea of primary mental healthcare implementation that GPs may not have appropriate clinical skills or mental health expertise to deliver this sort of care effectively (Fleury et al., 2012; Lester et al., 2005; WaMH in PC, 2015). Arguably, this is the reason why Matthew has not experienced any direct mental health input which he can express in his talk at any point in his interview. Patients with serious mental health issues may be more difficult to treat, requiring a more involved approach to tackle complex needs, which it has been argued is beyond the remit of the GP (Balanchandra et al., 2005; WaMH in PC, 2015). Thus, the metaphor of opening 'Pandora's box' has been invoked by some professionals to describe what is perceived as an intrusion of primary care into the mental healthcare domain (Lester et al., 2004). Potentially, this flies in the face of notions of parity of esteem between physical and mental healthcare (Health and Social Care Act 2012).

It is also noticeable in this passage that Matthew talks in quite reticent terms about actually seeking the GP's clinical care since the GP is often "busy" (line 122) and consequently at times there "isn't an awful lot he can do" (line 124). This latter statement also adds to the sense that mental health care and support is not being provided for Matthew in his current maintenance stage by the GP. A little later on in the interview, Matthew clarifies both the limited nature of current care provision from the GP, specifically in this sense of the GP being busy: 
237. Matthew: No, $<<$ CMHT $>>$ discharged me, you see, and also

238. the only reason I see my doctor now is for medical reviews,

239. and I just keep him in the picture when it's time for a form.

240. He doesn't fill out the form, he's a busy man.

[Matthew, Service user, Phase 1, Participant 9, lines 237-240]

This and other instances in Matthew's interview indicate a certain amount of reticence about him seeking healthcare from the GP. Matthew only now sees his GP once a year for reviews so can only "imagine he's got a busy life" (line 461). Consequently, Matthew "tends to let him get on with his job" (line 467). This restraint on help-seeking could be understood to be the result of a deterrent effect (Robertson, 2016) resulting from Matthew's perception of service pressures (lines 122 and 240). Matthew's deferential talk in relation to his perception of pressure on primary care services implies that this leads to him being less inclined to seek healthcare from the GP. There is support in literature on local services for my argument here that Matthew's perception of busyness and limited resources acts as a barrier to help-seeking and receipt of healthcare. The special interest group 'Wales Mental Health in Primary Care Network' (WaMH in PC, 2015) identified in their survey that limited service capacity is the third greatest barrier to the successful delivery of primary care mental health services. In addition, $88.5 \%$ of primary care workers in this survey said that there should be significantly greater investment in time and resources in mental healthcare in Wales. The view here is that service pressures might be relieved by investment in greater service capacity, and that this would be a key way to improve access and availability of care. Analysis of Matthew's talk may be interpreted as providing support for this view, since service pressures contributing to his GP's busyness appears to be a major factor for a deterrent effect which acts as a barrier to seeking healthcare.

Summing up this analysis, Matthew's account is the main exemplar in the data set of talk related to 'Experiences of practices promoting continuities of primary care'. Analysis of Matthew's talk appears at first to support various dimensions of care continuity. However, I would argue that closer analysis of talk leads to elucidation of various discontinuities of care which are embedded in his account. Additionally, instances of care continuity are fulfilled in a quite limited and one-dimensional way, which it appears are not specifically related to mental healthcare. Detailed qualitative analysis of talk related to the category 'Experiences of practices 
promoting continuities of primary care' therefore contributes in a paradoxical way to the theme 'Experienced discontinuities within primary mental healthcare'. In the next section I present further analysis of talk more obviously related to this latter category.

\subsection{Experiences of practices promoting discontinuities of primary care}

This category consists of concepts and talk related to experiences of activities and practices promoting discontinuities within primary care. A variety of concepts are collated under this category which are represented in the following figure:

\section{Figure 12: Experiences of practices promoting discontinuities of primary care}

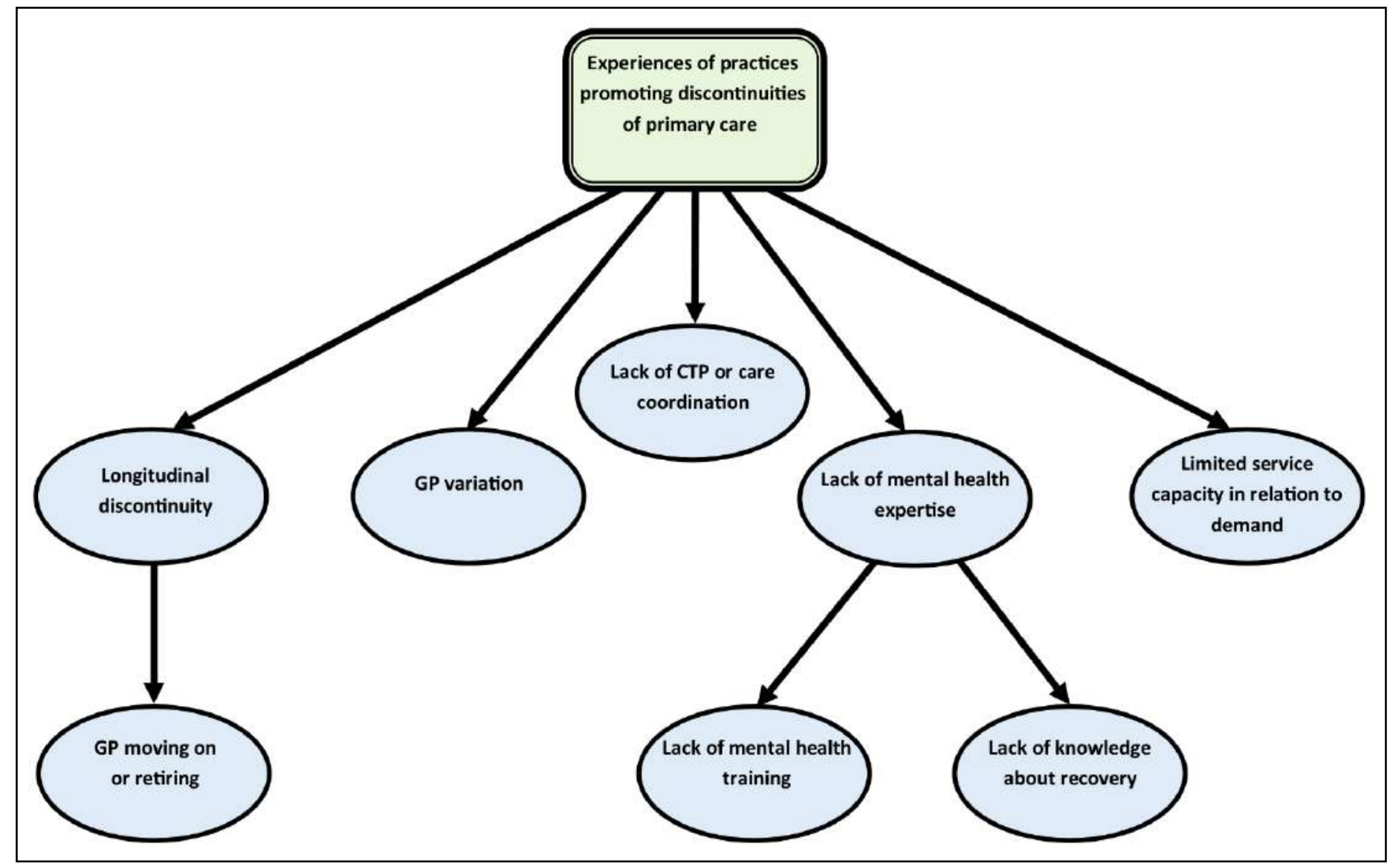

'Longitudinal discontinuity' (Burns et al., 2009; Ware et al., 2003), an area of discontinuity which has already been touched upon in analysis of Matthew's talk, is represented conceptually above and is talked about by $(n=8)$ participants. Talk collated under this concept and dimension of care continuity concerned difficulties participants experienced because of gaps 
in their treatment due to the unreliability of mental healthcare provision in the General Practice (concept 'GP variation'). This problem was talked about by participants where it was claimed that some GPs lacked confidence in providing mental healthcare. This supports the idea in the literature that some GPs may not have appropriate clinical skills to deliver mental healthcare effectively (Balanchandra et al., 2005; Fleury et al., 2012; Lester et al., 2005; WaMH in PC, 2015). Some gaps in treatment resulted when the service user preferred to wait until a GP became available with more mental healthcare expertise (related to concept 'GP variation'). More generally, a gap in treatment for the whole practice would be created when a GP who was capable in providing mental healthcare moved on to another practice or retired. Two participants, Tricia and Sheryl, talked about this experience, leading to my formation of the concept 'GP moving on or retiring' as a particular subset of longitudinal discontinuity in the concept map.

'GP variation' was specifically talked about by $n=10$ participants. This concept collates codes and talk about the difficulties participants encountered because of the variation in mental healthcare provision. This concept was distinguished from the concept a 'Lack of mental health expertise' in that it conveys the sense that mental health expertise was present in some cases but that the availability of such expertise was not consistently or reliably available.

A 'Lack of mental health expertise' was in the previous section related to potential disruption to good quality treatment with the potential to promote discontinuities of care (Balanchandra et al., 2005; Burns et al., 2009; Fleury et al., 2012; Lester et al., 2005; Rose et al., 2009; Sweeney et al., 2016; WaMH in PC, 2015). A number of participants $(n=8)$ considered that a major contributing factor to this deficiency in expertise was a lack of training in mental health (concept 'Lack of mental health training'). This perception tallies with the literature indicating that mental health training amongst primary care staff and GPs can often be absent (Currid et al., 2012; Freeman et al., 2002; Lester et al., 2004; WHO, 2008). Participants $(n=10)$ also specifically talked about a 'Lack of knowledge about recovery' within primary care, which could be regarded as a type of expertise about mental healthcare. Three of them were GPs who each admitted they did not have immediate knowledge about recovery theory and approaches. 
'Limited service capacity in relation to demand', which has already been identified as an issue for GP care availability in analysis of Matthew's account, was a major area of concern in my overall data set, talked about by $(n=11)$ participants. There was a perception among participants that the capacity for GPs to provide mental healthcare was inhibited by large caseloads which contrasted starkly with their limited service capacity to provide healthcare.

Finally, the concept 'Lack of CTP or care coordination' collated talk from participants $(n=$ 14) about the absence of a care coordinator or any approach of care coordination once transition to primary care had occurred. This transition would mean that the use of a CTP in planning care would cease, since it was not passed from secondary services care coordinator to the GP. In consideration of this issue, I consistently inquired in interviews about whether the participant was aware that the CTP, or information in it, had been passed from the care coordinator to the GP at the point of discharge. Participants were unanimous that this had not occurred to their knowledge. It seems from the data set that as far as participants using services were aware, the CTP approach is dropped abruptly as soon as secondary care ceases, along with potentially valuable care planning information.

In the following sections, I analyse talk in data extracts taken from three participants, Tricia, Susan and Maria. Through discourse analysis of their talk, building on conceptual analysis, I argue that there is a concentration of a variety of care discontinuities, within multiple dimensions of the concept, operating in and around the primary care level.

\subsection{1 "Well, they pass the buck then, don't they?" Expectations of the GP role limiting care continuity}

Tricia is a female service user participant in her mid-thirties suffering from anxiety and related psychotic episodes. The concept map I formulated from her coded interview transcript is provided as an example in Appendix 10. She had been discharged from secondary care within the past three years and was therefore suitable for recruitment in the first phase of data collection. In her research interview, taken as a whole, Tricia displayed a degree of ambivalence about having her care transferred to the GP. On the one hand, she states that she 
is happy to have been discharged since this has led to her feeling more empowered and in control:

470. Tricia: I feel quite happy to be discharged.

471. I felt it was an achievement after 25 years, to be

472. discharged, if you know what I mean?

473. Researcher: Why do you feel it's an

474. achievement?

475. Tricia: Because, I'm in control of my own

476. illness now.

[Tricia, Service user, Phase 1, Participant 6, lines 470-476]

Discharge had also resolved an impasse within her care pathway within secondary services where she was not progressing, a past situation she described as being "stuck" (line 499) shortly after the above data extract. I therefore understood Tricia to have a positive view of her status at having been discharged to primary care. However, despite this positive view of her status within primary care, the following extract displays a critical view of primary care provision itself:

219. Researcher: Yes, okay. So, with your GP, can

220. you give me an example of when that GP has referred you

221. to somebody who's been helpful for your treatment and

222. care, such as a psychologist, or a place like this, or

223. anything like that?

224. Tricia: Well, they pass the buck then, don't

225. they? They don't see you. You're referred to the people,

226. like the mental health team. I haven't been to the GP for

227. about a year.

228. Researcher: Okay, so do you feel that, actually,

229. sometimes referring you to somebody else isn' $t=$

230. Tricia: =It's better, it's better.

231. Researcher: It is?

232. Tricia: Yes.

233. Researcher: But, is it passing the buck, as you

234. just said?

235. Tricia: Yes, I suppose it is, but they're too

236. busy, the GPs, these days, to...(.4) Because, mental health is

237. complex, isn't it?

238. Researcher: Indeed.

239. Tricia: They haven't really got the time,

240. you know? Five minutes in with them.

[Tricia, Service user, Phase 1, Participant 6, lines 219-240] 
My initial line of questioning in this extract was based on question three in the semi-structured interview schedule for the first phase of data collection. This question, and associated prompts and probes, is predominantly an inquiry about participants' experiences and understandings of the ability of GPs to successfully connect them with services outside of primary care. This includes services which are not situated within secondary care, such as third sector organisations and also providers of psychotherapy. The analytical point I focus on here is that Tricia appears to take this line of inquiry as an opportunity to criticise the GP. The intention of my inquiry was to probe for positive experiences of cross-boundary continuity, where the GP might successfully connect the patient with other beneficial sources of care. It is crucial to note, in terms of Gee's (2010) view of language as organised around the basic structure of subject foci and predicates, that I at first attempt to focus this part of the interview on the GP's potential to instigate positive instances of cross-boundary continuity. Tricia's response to my focus on this issue is to construct a complaint and associated critique about the GP's capacity for crossboundary continuity. Tricia in a sense wrests the subject focus from my intention for it to be about positive instances of cross-boundary continuity, and turns the matter on its head by using my question as an opportunity to criticise the GP for a fundamental failure in undertaking crossboundary signposting or referral in order to successfully deliver quality care. She describes referral or signposting to another type of care as "pass [-ing] the buck" (line 224). This form of speech may be defined as "making someone else deal with something that you should take responsibility for" (Rundell and Fox, 2010). This idiomatic expression adds further emphasis to Tricia's rebuttal of my attempt to focus the discourse of the interview upon the positive potential for cross-boundary continuity within primary care. Gee (2010) points out that speakers choose subjects and their predicates strategically in the construction of discourse to determine the constructed version of reality they are advocating. Clearly, Tricia's construction of reality constitutes a negative evaluation of the GP's potential to stimulate and coordinate cross-boundary continuity.

This switch also represents an abrupt topic change from my initial focus, and therefore a disruption to a topic chain which would otherwise have occurred in this discourse. Whilst topic chaining may be used to build an ontological framework for the world (Gee, 2010), disruptions of potential topic chains may be used to refute potential ontology building. Tricia does this in terms of a potential ontology which my inquiry might engender about cross-boundary 
continuities of care between the GP and other services. In so doing, she refutes the credibility of an ontology about cross-boundary continuity around primary care which I am inviting her to construct. In disrupting my potential construction about care continuity, her talk implies care discontinuity. The use of metaphor in the idiomatic phrase "pass the buck" (line 224) is also employed to reinforce her disruption of talk attempting to construct a notion of care continuity. The metaphor is used as part of an idiomatic expression which has a special robustness contributing to the effectiveness of this discursive achievement. Since idiomatic expressions are not meant to be taken literally, they are resistant to being tested or challenged on an empirical basis (Drew and Holt, 1988).

These discursive devices combine to form a complaint (Drew and Holt, 1988; Edwards, 2005; Jingree and Finlay, 2013) from Tricia about the mental healthcare she receives from the GP. Tricia's understanding appears to be that rather than the potential for referral and signposting by the GP to other services being an opportunity for the enrichment of care provision through co-ordination of cross-boundary continuities, it is instead an opportunity for 'passing the buck' and not providing the best quality treatment. Referral and signposting can be a way of deflecting the patient to somewhere else in the system rather than providing treatment at the appropriate point of need (Robertson, 2016), or even rejecting the patient outright. The close proximity in the text to this idiom of the statement that she hasn't "been to the GP for about a year" (lines 226 to 227) can be seen as a meaning-making process in Tricia’s talk (Jørgensen and Phillips, 2002; Laclau and Mouffe, 1985) whereby she implies a connection between the perceived deflective activities of the GP and a deterrent effect which has led to her not seeking help from the GP for a long period of time. Deterrence from services can be a consequence of deflection as much as it can be a consequence of any activity which frustrates easy access to or inhibits the apparent availability of care (Robertson, 2016). For example, in the previous section I concluded from analysis of Matthew's talk that his perception of the GP's heavy workload and the limits this implies for care availability has led to his reticence in help-seeking. In Tricia's case, a perception of deflective activities on behalf of the GP may have led to deterrence from her making an appointment. She appears to position her account in this way by placing the idiomatic expression referring to deflection so close to her other statement at lines 226 to 227 about deterrence. From the perspective of care continuity, both of these statements imply both cross-boundary and longitudinal discontinuities of care. 
Tricia's talk in this data extract could be considered to be a rebuttal of the idea that the GP might fulfil a limited role of care coordinator, acting as a central point for coordination of multiple, disparate sources of care provision distributed throughout the community (Coffey et al., 2017b; Hannigan et al., 2018; Simpson et al., 2016). The understanding of this care coordinator role for the GP here would be quite limited and confined to the sense that the practitioner at least promotes some degree of cross-boundary continuity by way of signposting, referring and promoting links with other potentially helpful services. In my systematic review (Weaver et al., 2017), I note the close connection between cross-boundary continuity and care coordination. Given that Tricia's talk is constructed so as to critique potential cross-boundary continuity within primary care, this also has negative implications about her expectation that the GP might act as care coordinator, even in this more limited capacity.

It may be objected that GPs should not be expected to be care coordinators, since this is not their established role within primary care. However, if the issue of professional incongruity between the role of GP and care coordinator is not addressed (Durbin et al., 2016; Lester et al., 2004; WaMHinPC, 2015), and there is no other provision of care coordination at the primary level, the problem is not resolved as to how care continuity and coordination might be maintained for service users after discharge from secondary care, and during the maintenance stage of their illness. As Ramanuj and colleagues (2015) have noted, this deficiency within primary care may well be one of the major contributors to higher rate of crisis $(60.3 \%)$ amongst patients being referred back to secondary care. Tricia's talk, and related talk collated under the concept 'Lack of CTP or care coordination' in my conceptual analysis, would seem to indicate that there is a gap in terms of care coordination and associated cross-boundary continuity once discharge has occurred. Indeed, whilst participants' talk collated under the concept 'Lack of CTP or care coordination' is related to negative views about a scarcity of care coordination or care planning within primary care, Tricia goes further in her talk by being resistant to the idea that this kind of care should be conducted by GPs at all. This is inherent in the way in which she so vehemently rebuts in the discourse of our interview my attempt to construct an ontology of the GP as limited care coordinator.

Despite formulating this critique, it is notable that Tricia constructs a rationale for understanding why GPs might struggle to fulfil the role of limited care coordinator, leading to 
their deflective and deterrent behaviour. She does this by mentioning issues of the busyness of General Practices (line 236), and the short appointment times this may be related to (lines 239 to 240). This construction in talk suggests that the reason for deficiencies in care are not so much the GPs' responsibility as they are the result of external factors which have placed them under undue pressure (lines 235 to 236), namely that there is not enough service capacity to cope with demand and the complexity of mental health issues (line 237). There is support for this notion in recent literature which highlights front-line areas such as primary care, emergency and acute psychiatric departments as particularly exposed to excessive workloads and associated stress and burnout (Durbin et al., 2016; Terry, 2018; Thomas and Morris, 2016; Wilkinson, 2015). This may be due to increasing demand, administrative burdens and caps placed on resources, (Hannigan et al., 2018; Simpson et al., 2016; Wilkinson, 2015). Talk here was therefore collated under the concept, 'Limited service capacity in relation to demand' in my conceptual analysis presented in Figure 12. Discursively, this passage of talk has the effect of locating responsibility for the inadequacies of GP care provision beyond the scope of their power and control. In many ways, this adds to the certitude of her critique of GPs as potential mental healthcare providers, since the underlying factors for this inadequacy are located beyond the domain of human agency, and are embedded within external, structural factors which have been built up by a variety of agents and social forces over time, and may be difficult to change (Archer, 1995; 2003).

Summing up, Tricia's account may be understood as a complaint related to a pessimistic view of the potential for good care continuity in the primary sector, especially in the dimension of cross-boundary continuity. This is associated with a negative view about the potential for GPs to fill the gap in care coordination that is created upon transition from the secondary level. Despite this critique of primary care providers, she is ambivalent about the nature of her status of having being discharged to the primary level, since her talk in the first extract presented in this section shows she welcomes this position of newly found independence. In the next section, I provide analysis of a data extract from the interview with Susan, another service user participant from the first phase of data collection, who is more singularly critical of mental healthcare at the primary level. She raises more fundamental difficulties related to the idea of GPs being effective mental health practitioners. 


\subsection{2 "They don't have enough insight into mental health. They're not trained psychiatrists": Care discontinuities related to limitations in GP mental health expertise}

Susan is a female service user participant in her forties who has experienced depression and anxiety, with some positive psychotic symptoms such as hearing voices (see line 84 below). She was recruited from the same third sector organisation as Tricia. One feature of Susan's research interview is that she constructs her version of the identity of GPs as having good intentions about providing mental healthcare, as well as making the effort to listen to her and empathise with her. Despite these good intentions, she voices concerns which form a critique of the ability of the GP to provide good healthcare for people with SMI, due to the fundamental limitation of having an inadequate understanding of the nature of mental health and care. The following data extract is one key passage in which she voices these concerns:

73. Researcher: And what do you think about the

74. ability of other GPs for mental health?

75. Susan: I think they don't know enough

76. about mental health to help people properly.

77. Researcher: Mm, so what kind of knowledge is

78. this that they're lacking?

79. Susan: I think (.4) ((Laughter))...

80. $\quad$ Researcher: It's a bit of a testing question, but

81. it's interesting for me to clarify your thoughts, and your

82. experience.

83. Susan: I think they don't always

84. understand. With me, I hear voices, and see things. They

85. don't always understand that kind of thing. They don't have

86. enough insight into mental health. They're not trained

87. psychiatrists. I think it's difficult for them.

88. Researcher: They're not trained psychiatrists.

89. The other possibility for mental health treatment is for

90. someone to be a mental health worker, of one shape or

91. form. Would you even put a doctor within the category of

92. being of a standard of a mental health worker, or do they

93. fall short of that?

94. Susan: They fall short of that.

[Susan, Service user, Phase 1, Participant 7, lines 73-94]

Susan states her view in this data extract about GPs' limited, expert mental health knowledge primarily at lines 75 to 76 . When I press her for clarification of what she thinks this kind of knowledge is (lines 77 to 78), she utters a brief abortive phrase, "I think -" (line 79), pauses 
and then makes the affect display of laughter. The succession of these occurrences is telling from a discourse analytical perspective. Jefferson (1984) has analysed conversations in which people talk about troublesome or difficult circumstances and found a recurring pattern of producing an utterance and then laughing immediately afterwards. This set pattern identified in conversation analysis fits Susan's talk here since she produces an utterance, "I think" (line 79), followed closely afterwards by laughter. After this, she goes onto describe difficult circumstances of not being understood by a clinician when describing her symptoms of mental illness. According to Jefferson's (1984) perspective on this kind of pattern in talk, Susan is setting up her perspective on the topic of GP's expert mental health knowledge as something troublesome and difficult for her.

Susan then describes the GP as not having enough "insight" (line 86) into mental health. The word 'insight' is a loaded term since it is a key term within the domain and discourse of psychiatry, employed by psychiatrists to describe the inability of the patient to realise their state of mental disorder. In the light of Goffman's $(1955 ; 1971)$ theory on face-work, in which persons have an obligation to maintain a contract of mutual intelligibility in a world of perpetual social encounters, a lack of insight implies a breakdown of this social contract. Furthermore, a lack of insight signifies a failure to provide a convincing account for transgressing the bounds of social intelligibility (Scott and Lyman, 1968), which may happen when a psychotic episode within mental illness occurs. When a breakdown of social contract occurs between psychiatrist and patient, one possible outcome is that this will have coercive implications since this breakdown impedes a consensus between psychiatrist and patient about the presence of mental illness, whether or not treatment is required, and the precise nature of this treatment. In place of this consensus, the pre-existing imbalance of power between psychiatrist and patient is enlarged to the point where the patient is disempowered and the psychiatrist is in a dominating position. At this point, the psychiatrist alone is considered to have true insight into the nature of the mental health difficulty, and how it should be tackled. The psychiatrist can then necessitate treatment, which engenders the coercive state of affairs. To describe this, Beck-Sander (1998) has identified the notion of 'treatment compliance' as a key dimension emerging from the 'insight' construct, where the patient lacking insight must comply with treatment, whether or not they agree with it. If their disagreement is pronounced, 
coercive force is justified to implement the treatment, on the basis of the dimension of 'treatment compliance' and the notion of a lack of insight.

The term 'insight' is therefore a critical one within the field of psychiatry, and the sociological understanding outlined above, since it is fundamentally expressive of the power which the psychiatrist has over the patient. By choosing to specify that this term should not be applied to the GP, Susan de-legitimises (Berger and Luckman, 1991) the medical credibility and authority of this type of clinician within the psychiatric field. Immediately following this use of language, Susan clarifies this de-legitimisation with the statement, "they're not trained psychiatrists" (lines 86 to 87). One of the four strategies for legitimation identified by Van Leeuwen and Wodak (1999) is 'authorisation' where legitimacy is achieved by reference to authority of tradition, custom, law or persons in whom institutional authority is entrusted. By demarcating the GP from the professional authority of the psychiatrist, Susan's discourse has the effect of de-legitimizing such practitioners as a credible players within the field of psychiatry.

The difficulty surrounding GP credibility which Susan highlights here cuts right to the heart of the tension which exists in attempts to realise recovery-based services in the form promoted by the Mental Health (Wales) Measure 2010. This policy involves a recovery ethos seeking to expand the treatment of serious mental illness beyond the confines of specialist, secondary services, governed by the psychiatrist, and more fully into the domain of primary care, under Part 1 of the Measure. Primary mental healthcare can also be regarded as a recovery version as was seen in analysis of GP participants' talk in Chapter five. However, in Susan's talk, the GP does not have the legitimacy to perform a psychiatric role and provide recovery, even at the level of a mental health support worker (line 94). Her talk highlights a key difficulty with recovery-focused primary mental healthcare implementation. While primary care may be considered to have many strengths and features which could contribute to the field of mental health (Lester et al., 2004), there is also doubt about the ability of GPs to participate expertly in the mental health field (Lester et al., 2004; Wright et al., 2005). As I highlighted in Chapter one, the special interest group of the Royal College of General Practitioners in Wales conducted a survey which reported that $72.7 \%$ of primary care staff described mental health as 'difficult' or 'very difficult' to manage (WaMH in PC, 2015). Thielke and associates (2007) also point out that primary care and psychiatry may be qualitatively different since the former is organised 
to manage acute diseases and maintain public health, and not to detect and diagnose mental illness. This may hamstring various approaches and models of primary mental healthcare implementation (Durbin et al., 2016; Gask et al., 1997), especially those underpinned by a recovery ethos seeking to empower service users and clinicians who are not psychiatrists. This could be seen as a similar problem to the one facing Katy in Chapter four where the recoveryimplementation of the Measure seeks unsuccessfully to broaden the domain of psychiatric determination beyond that of the specialist mental health clinician to include the service user or the GP.

Analysis of Susan's talk highlights the concept of 'Lack of mental health expertise' in Figure 12 as an issue of fundamental concern for recovery-based primary mental healthcare delivery since, if GPs can never be clinical experts in the field of psychiatry, as her talk expresses, this will be an impediment to good quality, continuous care. Analysis of her talk shows how this contributes to cross-boundary discontinuities of care, which will in turn effect other dimensions of care continuity negatively, given its integral place within the entire, multi-dimensional care continuity construct (Burns et al., 2009; Weaver et al., 2017). In the next section, analysis of talk from the research interview with Maria, a third sector day centre manager, adds to the theme being developed in this chapter about primary care discontinuities.

\subsection{3 "We want people to be self-empowered and do self-management, but for many people taking away the CPN is very scary": Two competing versions of recovery at the primary and secondary levels}

The final participant whose talk I analyse in this section providing analysis of talk related to concepts in Figure 12 is, Maria, the manager of a third sector organisation in Wales. This participant is female and in her fifties. Talk in the following data extract may most clearly be identified with the view that a lack of care coordination or care planning at the level of primary care contributes to discontinuities of care (concept labelled 'Lack of care coordination or CTP' in Figure 12). The following extract is the key passage of talk articulating her critique in relation to this concept. It occurs right after I probe for her views on the merits or demerits of the primary healthcare context for people with SMI who have been discharged from secondary services. 
153. Maria: I heard from the mother of

154. somebody who's attended here a long time recently that

155. he was going to be discharged from services, and they

156. are really worried, the mum's really worried. Because in

157. the past he's done things like not taking his lithium and

158. stuffed it all in the back of the drawer, and got really

159. unwell. So, the whole thing with the CPN for me is that

160. the care coordination is effective and works, there is

161. somebody somewhere supporting that service user. Not

162. taking responsibility for them, because we don't want

163. that, we want people to be self-empowered and do self-

164. management, but for many people taking away the CPN

165. is very scary. Because they know they won't get an

166. equal service off their GP.

167. Researcher: Let me just play devil's advocate

168. slightly, one could say if somebody hopefully is

169. discharged to the primary care, their mental health might

170. have improved to the point that they're not in the state of

171. relapse where they have been before. In that sense, you

172. could argue that the person is actually well enough to be

173. cared for by the GP.

174. Maria: Absolutely.

175. Researcher: Is that the case, or are there

176. problems with that idea?

177. Maria: Well, I don't know, if the person is

178. well enough, if they're stable, if they have capacity then

179. that should be all right. Some of the most serious cases,

180. and this chap I'm talking about now is a very serious

181. case, my advice to the family was to fight against it,

182. because I felt that strongly about it.

183. Researcher: So in that situation you actually

184. felt that a discharge would be $=$

185. Maria: =would be detrimental, absolutely

186. detrimental yes.

187. Researcher: Do you have experience of a

188. number of cases where you've felt there's been a

189. discharge to primary care which was inappropriate?

190. Maria: Yes, how many do you want?

[Maria, Third sector manager, Phase 2, Participant 5, lines 153-190]

Maria's talk here commences with a brief narrative account (Riessman, 2008) of a client service user she considers inappropriate for discharge from specialist services. This is for reasons such as his long-term need for support (line 154), a history of non-compliance with medication (line 156 to 159 ) and being a "very serious case" (lines 180 to 181). The reported facts of this 
narrative account surround the use of an emotion category report about the client's mother's worry about the potential discharge (line 156). An emotional category is designed to have the discursive effect of enhancing the construction and worldview it is associated with. From the discursive psychological perspective within discourse theory, the issue is not so much about the meaning of the emotion as an experience for the individual, but rather the discursive accomplishment which is achieved by its use in the account (Wiggins, 2017). In Maria's narrative account, the emotional category of worry is employed to emphasise and bolster her view that transfer to primary care for this person is undesirable and detrimental. This is added to by the use of a similar emotional category of fear later on in the data extract when she says that "for many people taking away the CPN is very scary" (lines 164 to 165, my emphasis added). Both emotional categories are to do with fear and anxiety at the prospect of discharge from secondary services and removal of that apparatus of specialist support. This may be a valid concern to have about service users, but in this passage of Maria's talk the use of these emotional categories has the discursive function of building a case against the notion that primary mental healthcare is an appropriate place for treatment of people with SMI.

Maria's preference is for service users with SMI to be treated within secondary care under a care coordinator since, in her view, this approach is "effective and works" (line 160). Her professional background is working in children's care which she describes as rigorously planned and coordinated by social services, in view of safeguarding issues for young, vulnerable people. This ties in with consideration of risk and safeguarding which have often underpinned care planning approaches (Coffey et al., 2017a; Coffey et al., 2017b; Hannigan et al., 2018). She sees this approach as consistent with the mandatory appointment of a care coordinator and use of a CTP within secondary mental health services (Hannigan et al., 2018; Welsh Government, 2010b; 2010c). In accordance with this view, later on in her interview, she raises a concern about safeguarding and responsibility for someone who is vulnerable and might not have insight into a decline in their mental health:

325. Maria: ....The

326. other thing is, talking about people trying to get back in

327. themselves, if they become ill they don't want to,

328. because they're too ill to see it. So there's a whole issue

329. of care and responsibility that concerns me, if people are 
330. discharged, who's looking after them? Where's the

331. safeguard? There's a safeguarding issue here.

[Maria, Third sector manager, Phase 2, Participant 5, lines 325-331]

On the basis of this talk it can be seen that her perspective of an absence of obvious and rigorous care coordination, care planning and taking responsibility for people with SMI now discharged to primary care is the cause of much concern for Maria. This forms the basis for her critique of support and treatment delivered in this way. Maria states that service users "won't get an equal service off their GP" (lines 165 to 166). This is an ambiguous phrase since it could be interpreted either as a statement of fact or as an evaluative statement. Fairclough (2003) identifies accounts which merge facts with evaluations as one type of discourse drawn from the 'hortatory' genre. The 'hortatory' genre achieves the function of evaluation and prescription though often covertly because the distinction between factual statements and evaluations is blurred (Fairclough, 2003; Rose, 2013). In Maria's use of this phrase, there is a blurring between the factual sense of inequality that the primary care service is not substantively the same as secondary care, and the evaluative sense that primary care is not as good as secondary care (lines 185 to 186 ).

Despite this, Maria qualifies her support for a care coordinated approach with the concern that people be "self-empowered and do self-management" (lines 163 to 164 in the first data extract). She recognises that there is a potential conflict between having a care coordinator and fulfilment of a recovery ethos that emphasises empowerment and self-sufficiency. The recovery version Maria invokes in lines 161 to 164 sounds very similar to the one intended to be implemented under the Welsh Measure through reduced dependency on services and increased primary mental healthcare implementation (Welsh Government, 2010a; 2013; 2014c). Taking this lead, I prompt her further on this recovery version of self-empowered and self-managed care (lines 163 to 164) by asking whether some people with SMI might be well enough to maintain their own care in this way under the GP (lines 167 to 173). Maria agrees with this (line 174) since she recognises a potential impediment to recovery in her commitment to the CTP approach, because they might become overly dependent upon secondary services (lines 161 to 164). This leads her to adjust her position during the first data extract, starting at line 177. From lines 177 to 182 she delimits her recommendation of a care coordinated 
approach at the secondary level by dividing people with SMI into two groups. The first groups of people with SMI are "well enough" (line 178) to be maintained within primary care. However, it is necessary that the "most serious cases" (line 179), including the individual in her narrative account, be kept in secondary care under a care coordinator.

According to this analysis, I argue that Maria's talk helps to reveal a fault-line between two versions of recovery which are implemented under the Mental Health (Wales) Measure 2010. The Measure presides over a recovery version based on self-management through implementation of primary mental healthcare (Welsh Government, 2010a; 2013; 2014c). At the same time, the policy of mandatory care coordination and CTP under Part 2 of the Measure can be considered to be an implementation of an alternate version of holistic and co-produced recovery (Amering and Schmolke, 2009; Gilburt, 2013; Shepherd et al., 2014). This latter version emphasises self-management in collaboration with the care coordinator and an holistic approach under the eight areas of the CTP (Welsh Government, 2014c; 2015; 2016). Maria's talk reveals that these two versions are competing and are potentially at odds with one another, which is why she divides people in her talk into two groups. This dual-pronged nature of the Measure's recovery implementation potentially accentuates the gap between primary and secondary levels which would be felt keenly by individuals once they have been discharged to primary care (this was represented in the concept 'Gap' between primary and secondary service levels' in Figure 6). Indeed, it seems that there is one recovery version being promoted at the secondary level and another at the primary level. This leap into the abyss constituted by this accentuated gap is what is so "scary" (line 165) about discharge entailing taking away the care coordinator or CPN. If nothing else, this transition could certainly be experienced as a pronounced disruption or discontinuity in their care. A care trajectory involving more gradual withdrawal of specialist support might mitigate this problem. However, the absence of any clear provision of care coordination at the primary level, implied by analysis of Tricia's talk in section 6.3.1, does not indicate that any such gradual adaptation of care continuity is likely. This is confirmed by my analysis of the data set in this study which does not provide good support for there being a common practice of gradually reducing support at discharge. Conceptual analysis of participant data has revealed that very often they experience a poorly managed process of discharge from secondary services to primary care (see Figure 5 in Chapter four: 'Discharge to primary care across the service system interface'). 
In sum, initially Maria's talk can be interpreted as a promoting a critique about practices promoting discontinuities of care due to a lack of care coordination at the primary level. However, further analysis of her talk reveals a more fundamental discontinuity related to a gap between secondary and primary levels which is exacerbated by two alternate recovery versions being implemented by the Mental Health (Wales) Measure. In view of this analysis, I have argued that the Measure involves two competing versions of recovery working towards different goals and in so doing opens the possibility that continuity and coordination as requirements of recovery-focused care at this service interface might be more limited than expected. Additional analysis of participants' talk has therefore indicated a further way that recovery-implementations promoted by the Measure may imply discontinuities of care, further contributing to the theme 'Experienced discontinuities within primary mental healthcare' being developed in this chapter.

\subsection{Self-management recovery within primary care}

In this next section, I examine talk and experiences amongst participants about the selfmanaged recovery version within primary care. Figure 13 below shows conceptual analysis under the category 'Self-management recovery under primary care' which represents this recovery implementation encouraged by the Mental Health (Wales) Measure 2010. This conceptual analysis collates codes which are related to participants' talk about their experiences of attempting to self-manage their care after discharge to the primary level. The following figure shows that conceptual analysis of talk about self-management within primary care generates codes primarily related to care discontinuities. Conceptual analysis therefore indicates that care discontinuities are a feature of primary care according to participant experiences. Building on this conceptual analysis, discourse analysis of related talk in this section will add to the argument that care continuities are concentrated around experiences of self-management after discharge to primary care. 


\section{Figure 13: Self-management recovery within primary care}

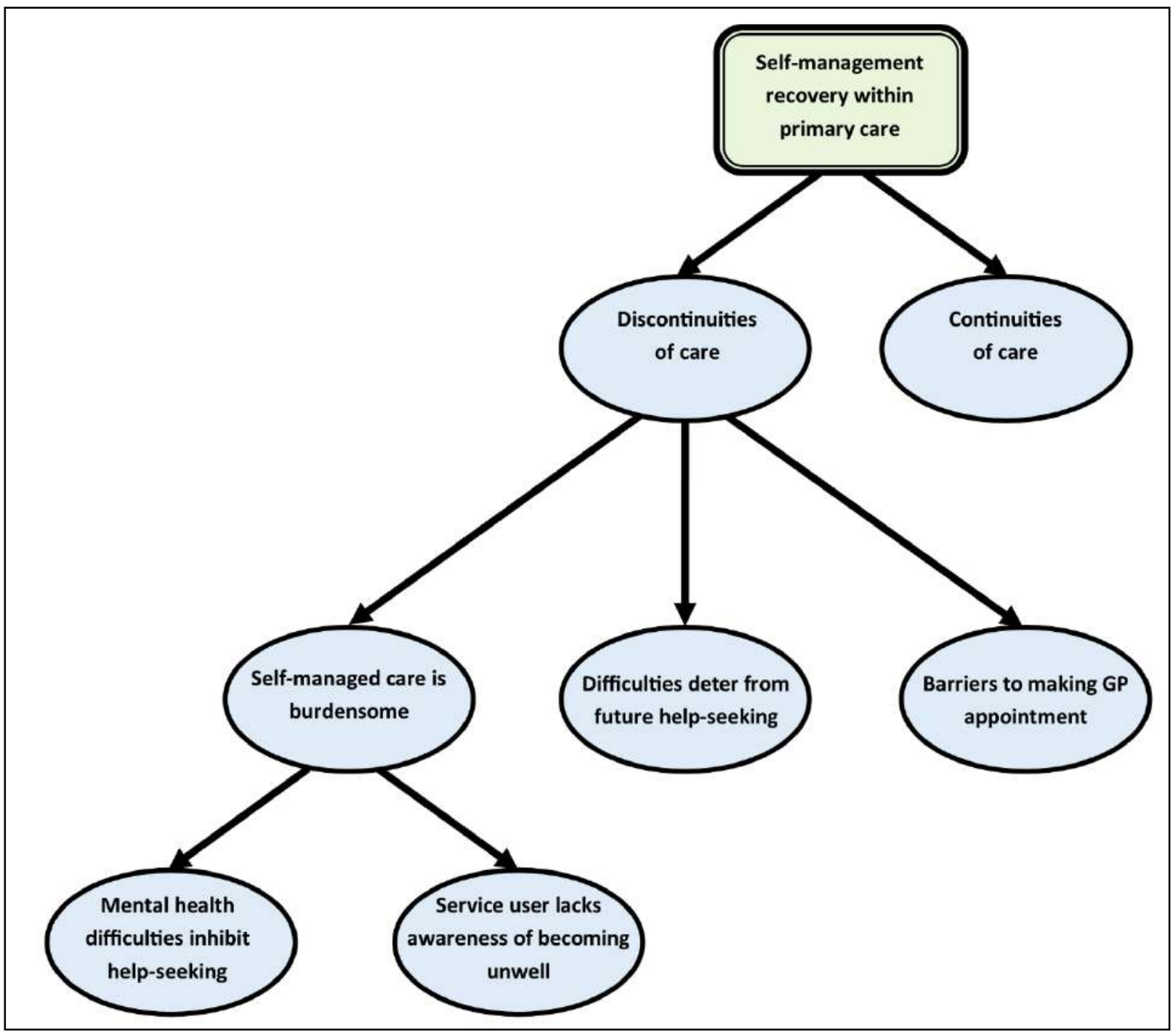

As can be seen from Figure 13, participants spoke about both continuities and discontinuities of self-managed care at the primary level, but the balance within the data was tilted firmly in favour of discontinuities of care, with participants $(n=21)$ speaking of discontinuities of one form or another, and $(n=10)$ participants talking about continuities. There was also a considerably greater number of concepts generated in relation to discontinuities of care than for continuities of care for participants self-managing at the primary level.

Talk collated under the concept of continuities of self-management in primary care included Tricia's preferred independent state after discharge from secondary services, which was 
discussed in section 6.3.1. Since Tricia described herself as previously "stuck" (line 499) within secondary services, the transfer to primary care and a greater level of independence had resolved a significant discontinuity in her care trajectory. Other participants from the first phase of data collection talked in similar ways about being in a position of greater empowerment in relation to their care, with the ability to choose a GP or even not to see a GP at all. The latter phenomenon could be regarded as an example of a continuity dimension labelled 'avoiding services' where it is considered to be a contribution to continuity if a person has the ability to decide to not have contact with a particular service provider if they should so choose (Rose et al., 2009; Sweeney et al., 2012; Sweeney et al., 2016).

With regard to discontinuities of care related to self-managed primary care, two notable concepts produced by conceptual analysis were labelled 'Mental health difficulties inhibit helpseeking' and 'Service user lacks awareness of becoming unwell'. The first of these referred to talk from $(n=3)$ participants about mental health difficulties inhibiting service users' ability to contact the GP. For example, some service users were spoken of as too anxious to pick up the phone to make an appointment or to leave the house to visit the practice. The second concept referred to talk from $(n=7)$ participants about difficulties service users have in realising they are becoming unwell. Since in primary care it is the responsibility of the service user to make an appointment, this phenomenon could represent a considerable barrier to requisite care. Related to both these concepts was the further concept that the responsibility to self-manage one's own care under the GP might be potentially burdensome for the service user (Dickerson, 2006; WHO, 2008).

Despite these and other issues, participants $(n=10)$ did talk in terms of self-managed recovery under the GP promoting continuities of care. One such participant was Sheryl, whose talk I now analyse. However, I will argue that close discourse analysis reveals care discontinuities as well as the more apparent care continuities related to Sheryl's experiences of individuals selfmanaging after discharge to the primary level. 


\subsection{1 "I think there's some good examples and there's some not so good examples": \\ Experienced continuities and discontinuities for self-managed care at the primary level}

This next data extract is taken from the semi-structured research interview conducted with Sheryl, a senior manager within a major mental health third sector organisation. In her research interview she focused on promotion of a person-centred, holistic recovery version which she claims is practiced by her own organisation. Following on from this, in the data extract below, I raise the query that this recovery approach might be appropriate for the person who has been discharged and now self-manages under primary care (lines 340 to 346):

340. Researcher: What about the process under the GP, the 341. person has to actually really have the impetus to actually

342. contact the GP. Have you perceived that to be either

343. problematic in the one sense or has it been a positive

344. thing in the sense that it's a good example of the

345. recovery model because this person's having to take

346. responsibility for their care?

347. Sheryl: Erm, (.), I think there's some good

348. examples and there's some not so good examples, you

349. know, I have seen somebody that has gone to their GP

350. when it's been hard for them to go and the process has

351. been made relatively easy. They've been able to get an

352. appointment relatively quickly. Their GP has actually

353. made them think that they've been listened to, and then

354. there's been a course of action. Whatever that might be.

355. There are GPs who are like that.

356. Researcher: So easier than it would normally be, you

357. know, the person hasn't had to phone up by 8:30 in the

358. morning...

359. Sheryl: Yeah, but there are those examples as

360. well.

361. Researcher: That happens as well?

362. Sheryl: Yeah, and there's some that, you

363. know, will say they can't get an appointment for five

364. days, you know. And that also relates to if you have - I

365. don't need to tell you, Nick, that some people with mental

366. health issues, it's quite heavily trust and confidence

367. based. So if somebody for example has had a GP for a

368. while but they feel really confident and have some trust

369. in then if they are unable - and they only can get an

370. appointment with that person ten days away, they will

371. take the ten day away appointment rather than actually 
372. see somebody quicker because they don't know that

373. doctor or they've heard that that doctor doesn't

374. empathise with people with mental health problems or

375. whatever. So there is - you know, it's also about the

376. individual themselves, but I do think that that's not

377. necessarily taken into consideration quite as much as it

378. should be. Bearing in mind that GPs are seeing

379. thousands of people aren't they?

[Sheryl, Third sector area manager, Phase 2, Participant 11, lines 340-379]

Sheryl's talk from lines 347 to 355 was collated under the concept of continuities of care within self-managed primary care in Figure 13 since here she refers to positive examples of individuals maintaining care and treatment from their GP. This has even been the case for patients who find it hard to go to their GP (lines 349 to 350), because the process has been made easier for them (lines 350 to 352). However, in line 355 she qualifies this by associating these positive examples only with some GPs. That this is not the case for all GPs creates a problem with longitudinal continuity (Biringer et al., 2017; Burns et al., 2009; Ware et al., 2003) since people with SMI may prefer to wait longer for an appointment with the GP whom they perceive can provide good mental health care (lines 367 to 375). It is true that this same difficulty of a gap in treatment due to having to wait for a 'good' practitioner may well be encountered by all patients of GPs, including those with physical complaints. However, these difficulties may be considered to be more pernicious and causative of experienced discontinuities of care for people with SMI, for whom an empathetic therapeutic relationship with the practitioner is paramount. Sheryl supports this notion that the therapeutic relationship, or its equivalent, relational continuity (Belling et al., 2011; Burns et al., 2009), is vital in mental healthcare by saying that it is "heavily trust and confidence based" (lines 366 to 367) and that service users will avoid a doctor that doesn't "empathise with people with mental health problems" (line 374). In support of this, Simpson and colleagues (2003; 2016a) comment that mounting evidence from service users and carers indicates that an empathetic therapeutic relationship is the key instrument in facilitating recovery. Sheryl's assertion that there are deficiencies in primary care of the kind of empathetic care that would qualify for relational continuity is also supported by a recent series of non-academic surveys conducted in Wales by the third sector organisation 'Gofal'. These initially identified a 'stubborn' 20-25\% of GPs who are regarded by service users as only 'slightly' or 'not at all' understanding and empathetic 
concerning mental health issues in 2014 (Gofal, 2014; 2013). Sheryl's talk about good continuity of care must therefore be qualified with a recognition of the presence of discontinuities of care associated with a sub-group of GPs (lines 373 to 374) which manifest within the relational and longitudinal dimensions of continuity.

Finally, Sheryl reinforces her account by stating that GPs deal with very large numbers of potential patients (lines 378 to 379). In a similar way to Tricia's account, this has the effect of reinforcing her critique of self-managed care at the primary level. The statement implies a limited capacity for GPs to offer the kind of in-depth involvement that might be appropriate for complex mental healthcare, and that this limitation is beyond the scope of their power and control, locating the source of the problem beyond the domain of human agency and instead within external, intransitive social structures (Archer, 1995; 2003; Danermark et al., 2001). The statement also has the effect of mitigating GPs' culpability, and accomplishes the task of avoiding being seen as overly confrontational, instead engendering a sense of her being sympathetic and therefore the creator of a more balanced and objective account. This can therefore be seen as a form of stake inoculation (Wiggins, 2017).

Returning to Figure 13, it can now be seen that Sheryl's talk should be interpreted to contribute towards concepts and categories about discontinuities of care such as 'Barriers to making GP appointment', as well as to concepts and categories about continuities of self-managed care at the primary level. The data extract in the next section taken from the interview with Bernadette in the first phase of the research project involves talk which provides additional support for concepts of discontinuity within self-managed primary care.

\subsection{2 "You need to see a doctor, and sometimes you can be desperate": Lived experience of discontinuities within self-managed primary care}

Bernadette is a service user in her forties diagnosed with a bipolar condition who was recruited in the first phase of data collection within a third sector organisation. During the course of her research interview she conveys a generally negative view about self-managing her mental healthcare under the GP. The following data extract is one of passages in this account which relates to the above concept map. It follows my inquiry and probe about the practical issues 
related to making an appointment for mental healthcare from the GP, which would be an important potential undertaking for someone self-managing their mental health at the primary level:

227. Researcher: Another thing I'm

228. interested in is actually getting an appointment with your

229. GP, for [mental health, because you -

230. Bernadette: That's hard.] That's hard. Because

231. you've got to phone up to half past eight, and then

232. sometimes they'll say, "A nurse will phone you back",

233. when, you know, you're desperate sometimes. You need to

234. see a doctor, and sometimes you can be desperate. Well, it

235. just puts you off phoning the GP because they'll say,

236. "Somebody will phone you back", or sometimes you have

237. to wait in a queue. Like, if you phone on a Monday, it's so

238. busy with patients that you can't see a doctor, you know,

239. and what happens when you're really ill?

240. Researcher: Yes, so that's a difficult, actually

241. getting the appointment itself.

242. Bernadette: Yes, and it puts you off, then,

243. phoning for the appointment.

244. Researcher: Have you ever tried actually going

245. there physically, to Reception?

246. Bernadette: No, I've never tried that, no. I

247. always phone up first to make an appointment.

248. Researcher: When you've seen your GP, over

249. the past two years, have you always had the same GP?

250. Bernadette: No, different ones all the time.

251. Researcher: Does that cause any problems?

252. Bernadette: Yes, because they don't know my

253. hist- (.2) well, they probably do know my history, because it's

254. on the computer, or whatever, but they don't know me, if

255. you know what I mean, and it does, you know, cause

256. problems.

[Bernadette, Service user, Phase 1, Participant 3, lines 227-256]

Bernadette's account conveys in terms of the immediate lived experience of a service user the kinds of discontinuities of care already touched upon by Sheryl, and represented in the labelled concepts of Figure 13. Emotional category terms are employed such as "desperate" (lines 233 and 234) and "hard" (line 230) in order to describe the difficult experience of being mentally unwell and having to adhere to a highly structured practice appointment protocol. This involves requirements to phone the practice for an appointment before 8:30 in the morning, and to wait for the practice nurse to phone back even after contact has been made. As has been seen 
previously in this chapter, busyness is viewed as an issue which limits care availability (line 238), and a difficulty which may deter from help-seeking. Other discontinuities are alluded to such as practitioner variation (line 250) and impediments to building relational continuity with a practitioner who knows her well and understands her mental health condition within this perspective (lines 252 to 256). In Figure 13, these "problems" (line 256) are represented by concepts of discontinuities of self-managed care under the GP such as 'Barriers to making GP appointment' and 'Mental health difficulties inhibit help-seeking'. The procedural difficulties she encounters in particular can be seen as objective barriers to care continuity. The problematic and "hard" (line 230) nature of conducting herself so as to progress along this care pathway can, according to Bernadette, lead to a state of desperation (lines 233 and 234). This supports the idea that self-management under the GP can be burdensome (see concept 'Selfmanaged care is burdensome' in Figure 13), a concern which is supported in the literature (Dickerson, 2006; WHO, 2008). Finally, in line 242, Bernadette describes a deterrent effect which has occurred for potential future help-seeking resulting from the difficulties she has encountered (Robertson, 2016), which was one instance of talk collated under the concept 'Difficulties deter from future help-seeking'.

As with Sheryl's account, it may be objected that these various difficulties and impediments to continuity of care described by Bernadette are experienced for all patients receiving primary care. The key issue is the particular kind of effect which these difficulties may have for people with SMI. Since mental healthcare is often complex and rooted in a wide distribution of social, psychological and physical factors (Crawford et al., 2004; Weaver et al., 2017; WHO, 2014), care continuity may be perceived to be a more fragile phenomenon in the case of people with SMI. People with SMI may find that their ability to pursue and maintain care is more sensitive to the deterrent effect of barriers to care continuity, and the disruptive effects of other discontinuities of care, such as the ones described by participants. Conditions such as anxiety and depression may lead to augmented negative responses to obstacles, which can be debilitating, especially when they are exacerbated by adverse situations. Additionally, serious mental health conditions can deteriorate very quickly without early intervention leading to the point of crisis (Becker et al., 2019; Brooker et al., 2007; Forrest, 2004). Consequently, it seems that the usual impediments to accessing primary care may have a disproportionate effect on people with mental health problems, putting them off from help-seeking, because they may 
give up more easily and their conditions can deteriorate very quickly leading to hospitalisation. Overall, therefore the picture created by conceptual and discourse analysis of talk related to a self-management version of recovery within primary care is that there are more discontinuities than continuities of care in the immediate lived experience of people with SMI, such as Bernadette, which provides further support for the theme 'Experienced discontinuities within primary mental healthcare'.

\subsection{Conclusion}

In this chapter, I have developed the theme 'Experienced discontinuities within primary mental healthcare' through qualitative analysis indicating a preponderance of discontinuities of care with regard to participants' talk and experiences of primary level services. This has been done in a variety of ways. Analysis of Mathew's talk showed that apparent continuities of care framed within a promotional account can mask underlying discontinuities. Tricia's talk expressed a complete rebuttal of the idea that GPs might be able to provide genuine mental healthcare in the form of limited care coordination involving signposting to other services. Analysis of Susan's account revealed a key challenge to the notion that the GP is a credible mental health clinician with the necessary level of expertise to deliver continuous care. This highlights an underlying difficulty for recovery-based movement away from specialist care towards self-managed or primary care-led mental health provision, which is entailed in the recovery version being promoted by the Mental Health (Wales) Measure 2010. My analysis of Maria's talk highlighted a fault-line between two competing recovery versions under the Measure being implemented at both secondary and primary levels, which exacerbates the gap between primary and secondary levels. Finally, with regard to one of these recovery versions, self-managed care within primary care, Sheryl and Bernadette's talk showed that various impediments and barriers to good care continuity affect this approach.

Through analysis of talk, in this and previous chapters, experiences of care discontinuities have been identified in many of the dimensions indicated in my published systematic review of care continuity (Weaver et al., 2017), and at multiple points and interfaces within Welsh service structures. However, what analysis in this chapter has shown is that care discontinuities are concentrated at the primary level and are also a key feature of self-managed care under the GP, 
which the Measure seeks to promote as a version of recovery. Additionally, I have argued that analysis has shown that the discontinuity or gap which occurs at transition across the interface from secondary towards primary care is potentially exacerbated by implementation of the Measure. The theme of this chapter has therefore been of 'Experienced discontinuities within primary mental healthcare'.

In the next chapter, I provide discussion and theoretical analysis of the findings of this and the previous two chapters. This forms the basis for me to perform theoretical generalisation and provide a series of cogent and cohesive explanations about the main research phenomena of this inquiry. 


\section{Chapter 7: Recovery and complexity in mental health services in Wales: a postpsychiatric and Habermasian perspective}

\subsection{Introduction}

In this penultimate chapter, I provide a synthesis of my analysis and findings established in the previous three chapters to form the main argument for this thesis. I have made use of various theoretical strands in the course of preceding analysis based on complex systems theory, recovery, and care continuity theory. In this chapter I synthesise these theoretical directions to provide an integrated analysis which builds on themes and findings in previous chapters. This integrated explanation is the basis for theoretical generalisation undertaken in this chapter and, following on from this, study implications in the concluding chapter. My approach to theoretical generalisation is described in section 3.7.1, and is based on the understanding provided by Mitchell (1983) and Seale (1999), as opposed to the more common understanding based on grounded theory (Glaser and Strauss, 1967; Corbin and Strauss, 2008; Strauss and Corbin 1990). In section 3.7.1, I described my approach to theoretical generalisation as a cogent explanation of findings which does not incorporate the notion that a complete theoretical analysis of research phenomena, or a substantive theoretical explanation may be reached (Corbin and Strauss, 2008; Strauss and Corbin 1990). Therefore, I do not consider the theoretical explanation provided in this chapter to be somehow the final word on the concepts and themes which have arisen out of this thesis, and I believe it would be possible to take another theoretical understanding into uncharted territories which has not been visited by this particular piece of analysis and theoretical argumentation.

This chapter is structured as follows. In the next section, I provide a recapitulation of findings, themes and theory developed in previous chapters. In subsequent sections, I first focus on misaligned expectations of services, which was the finding of Chapter four. I argue that the poststructuralist theoretical underpinning for discourse theory that I introduced in sections 3.6.4 and 3.7.1.2, in relation to Laclau and Mouffe (1985), provides a rationale for how misaligned expectations, as a feature of discourse, are a complex systems effect. After this, I develop theory surrounding the theme and main finding of Chapter five, namely 'Competing versions of recovery'. I expand upon my application of Laclau and Mouffe's (1985) 'floating 
signifier' in section 5.4.1, which sits within their poststructuralist discourse theory, as a way of describing the polyvalent nature of the recovery concept. I argue that this leads to a further understanding of how complexity may be generated in the social context of recovery-based mental health services. I also introduce my concept of 'recovery trajectory' in section 7.7 which I use to relate discursive complexity about misaligned expectations and recovery to more objective phenomena in participants' talk about experiences of care continuities and discontinuities. By employing these key theoretical perspectives, I argue that escalating mental health service system complexity can be understood as emanating from discursive behaviour surrounding misaligned expectations and competing recovery versions.

In section 7.8 , I broaden this picture by relating it to a postmodern or postpsychiatric view of Welsh mental health services characterised by complexity and fragmentation (Bracken and Thomas, 2001; Bracken et al., 2012). In sections 7.9 and 7.10, I argue that the Habermasian theory of system versus lifeworld (1984a; 1984b; Crossley, 2005; Pusey, 2002) provides a key theoretical understanding of the way in which recovery is often colonised, which I introduced in Chapter five. This leads to a focus on neoliberalism as a prevailing colonising and hegemonic (Crossley, 2005; Gramsci, 1971; Laclau and Mouffe, 1985), force affecting recovery. This is primarily seen in the form of policy-programmes, such as the Mental Health (Wales) Measure 2010 , which promote a narrow interpretation of recovery as a return to work and reduced dependency on services and welfare (DoH, 2009; Department of Health and Human Sciences, 2003; Gilburt, 2013; Mind 2008; Ramanuj et al., 2015; SAMHSA, 2004). I argue that this form of neoliberalist colonisation acts as a particular catalyst for service complexity, as well as being distortive of the radical idea of recovery originally rooted in the lived experience and lifeworld of service users and survivors. I argue that a key structural dichotomy underlies competing versions of recovery, between a top-down systemic, neoliberalist recovery type and a bottomup emancipatory recovery type rooted in the lifeworld of the mentally ill person. I make use of Habermasian social theory applied in this way as the basis for study implications in the concluding chapter, where I propose potential resolutions for the problems of escalating complexity, service fragmentation and potentially distortive neoliberal recovery versions. 


\subsection{Summary of findings in previous chapters}

I will now provide a review of the main findings in the previous three chapters. In Chapter four, I constructed the theme 'Misaligned expectations in negotiating transforming services' which describes how participants' talk expresses misaligned expectations in negotiating service systems structures and interfaces. Misaligned expectations were particularly manifest in analysis of participants' talk about negotiating the interface between primary and secondary care. For instance, my analysis of James, Alun and Katy's accounts revealed discursive positioning in relation to the expectation that they should have greater access to secondary services. This expectation ran counter to or was misaligned with the recovery-oriented shift within services discouraging dependency upon specialist care and encouraging selfmanagement after discharge to primary care. I understood the Mental Health (Wales) Measure 2010 to be a key factor for this shift of emphasis within services (Welsh Government, 2010a; $2013 ; 2014 \mathrm{c})$. However, as I argued in section 1.2, I consider the Measure itself to be a product of the general trend towards recovery-based services. Therefore, this paradigmatic trend can be considered to be the underlying factor stimulating misaligned expectations of transforming service structures. Within this context, I argued 'Misaligned expectations in negotiating transforming services' should be understood in the light of complexity theory as a 'wave of consequence' emanating from a solution, in the form of the Measure, to the 'wicked problem' of implementing recovery-based mental health services in Wales (Hannigan and Coffey, 2011; Rittel and Webber, 1973; Welsh Government, 2010a; 2014c; 2015).

In Chapter five, my analysis of participants' talk generated the theme 'Competing versions of recovery'. Versions of recovery were competing since they were often positioned in opposition to one another within discourse. For instance, the third sector recovery versions of Tony and Hannah were positioned in opposition to nascent primary care recovery versions provided by the three GP participants, Robert, Alex and Tamsen. In addition to the analysis of Robert's talk provided in Chapter five, Tamsen also talks about parallels between the recovery approach and key features of primary care, which I consider to be a nascent primary care recovery version. The following data extract taken from my interview with Tamsen follows my inquiry about the scope that there might be for recovery within a primary care. I employ the oft-quoted definition of recovery from Anthony (1993) as a prompt to which she responds: 
376. Tamsen: So what you've just described there is something

377. we would probably be aiming for with all our patients so,

378. you know, when we think of our patients we think of them

379. as living with their diagnosis rather than suffering from their

380. diagnosis so you know, see you know living with something

381. and as you say adapting your life in terms of actually $-(.2)$ and

382. possibly changing some aspects of your life so you're

383. actually able to leave - live as full a life as you can despite

384. having whatever condition you have, and that's the same

385. for any, any medical problem you [might have be that

386. Researcher: [Physical...

387. Tamsen: [Physical as well, absolutely. You know, so we

388. have patients with $\mathrm{COPD}^{18}$, you know so there are limitations

389. to what they are able to do physically, but that's not to say

390. that they can't live a full and happy life, despite those

391. limitations. So it's about adapting.

[Tamsen, General Practitioner, Phase 2, Participant 14, lines 376-391]

According to Tamsen, it is well-established within primary care that for chronic conditions such as COPD, and actually for healthcare in general (line 377), the focus should not be on the illness but on helping the patient to live life as fully as possible (line 383 and line 390), in spite of the limitations caused by illness. This describes the person-centred approach in primary care, focusing on symptoms in the context of the person's life and social milieu, rather than viewed through the filter of the medical model (Currid et al., 2012; Lester et al., 2004). It also accords with many tenets of recovery (Amering and Schmolke, 2009; Copeland, 2000; Repper and Perkins, 2003), as well as with the definition of recovery I provide her with from Anthony (1993).

However, despite the potential to adapt pre-existing primary care approaches for chronic physical health conditions to mental health recovery, and a degree of collaboration between the third sector and primary care, my analysis in Chapter five focused largely on impediments to cooperation revealed in talk of participants such as Tony and Hannah. Partly, this was due to such collaboration not yet being well-established, as was expressed through talk collated under the concept 'Collaboration less established than with secondary care' in Figure 10. However, I

\footnotetext{
${ }^{18}$ Chronic obstructive pulmonary disease (COPD) is a common lung disease which makes it hard for the person to breathe. It occurs in two forms, chronic bronchitis and emphysema, and is primarily caused by exposure to cigarette smoke or other noxious fumes.
} 
argue that the main reason for this oppositionality is because generated recovery versions between these sectors are often competing.

Additional evidence for competing versions of recovery was presented in analysis of talk of participants such as Alun who constructed multiple recovery versions within a single account which contradicted and competed with one another, arguably impeding his progress to wellness. Dylan and Maria were other participants for whom analysis indicated multiple, competing recovery versions co-existing within their own talk (see section 5.2.2 and section 6.3.3). Indeed, a key feature of my analysis in Chapter five was the identification of multiple instances of colonisation of the recovery concept in participants' talk. I argued that separate instances of colonisation were a major factor behind construction of competing versions.

I identified a number of types $(n=7)$ of colonisation within my analysis, occurring at multiple instances within participants' talk. These types were as follows:

1. Colonisation by a neoliberal policy agenda of work-as-recovery (constructed in relation to discourse analysis of Alun's talk, see section 5.2.3; associated with concept 'Productive and occupying work' ( $n=4$ participants), under category 'Recovery approaches and constructions', see Figure 7).

2. Colonisation by a neoliberalist ideology of self-sufficiency and responsibility (see particularly the concept 'Empowerment and self-management' ( $n=6$ participants), under the category 'Recovery approaches and constructions', see Figure 7. I have argued that the Mental Health (Wales) Measure 2010 is a policy-based construction of this recovery version, see section 2.6.3).

3. Colonisation by an emancipatory programme of self-management in which coercive and potentially iatrogenic services are avoided (constructed with discourse analysis of Dylan's talk, see section 5.2.2; associated with concept 'Empowerment and selfmanagement' ( $n=10$ participants), under category 'Recovery approaches and constructions', see Figure 7). 
4. Colonisation by a paradigm of holistic medicine (constructed with discourse analysis of Dylan and Sheryl's talk, see sections 5.2.2 and 6.4.1; associated with concept 'Holistic care' ( $n=31$ participants), under category 'Recovery approaches and constructions', see Figure 7).

5. Colonisation by pre-existing care and support practices of the third sector such as psychotherapy or rehabilitation (constructed with discourse analysis of Tony's talk, see section 5.3.1, and Hannah's talk, see section 5.4.1).

6. Nascent primary care recovery version colonised by some of the features and principles of primary care (constructed with discourse analysis of the talk of GP participants, Robert, in section 5.4.2, and Tamsen in this section, and conceptual analysis including Alex's talk collated under the concept 'Nascent primary care-based recovery' $(n=3$ participants), under category 'Recovery approaches and constructions', see Figure 7).

7. Medicalised colonisation of recovery-as-cure (constructed with analysis of Dylan's talk, see section 5.2.2).

In section 5.4.1, I also introduced the concept of the 'floating signifier' from the discourse theory of Laclau and Mouffe (1985) within analysis of Hannah's talk about recovery which I argued had wider applicability to more general instances of recovery appropriation.

In Chapter six, analysis of talk revealed experiences of care discontinuities in multiple dimensions of the multi-dimensional framework for care continuity (see section 2.7). Analysis in earlier chapters had indicated experiences of care discontinuities as a recurrent feature at various points within complex and fragmented services illustrated by my conceptual mapping of participants' experiences of services. However, analysis in Chapter six identified a concentration of care discontinuities at the primary level. This was a pertinent finding given the impetus intended to improve care delivery under the Mental Health (Wales) Measure 2010 towards decreased dependency on specialist, secondary services and increased selfmanagement after discharge to primary care (Welsh Government, 2010a; 2014c; 2013). 
In addition to this, a key discontinuity or 'gap' between primary and secondary levels was highlighted in analysis of Maria's talk (section 6.3.3), which could be related to conceptual analysis (see concept 'Gap' between primary and secondary service levels' in Figure 6). Through analysis in section 6.3.3, I argued that a pre-existing structural discontinuity between primary and secondary levels has been exacerbated by the implementation of two separate recovery versions under the Mental Health (Wales) Measure 2010. My analysis in Chapter six contributed to an emerging picture of Welsh mental health services characterised by complexity, fragmentation and discontinuity, which to an extent is related to implementation of primary mental healthcare delivery understood as a recovery approach.

In the next section, I commence my argument for theoretical generalisation by first revisiting one of the principles I laid out for this process in section 3.7.1.2, namely the poststructuralist position of discursive universalism adopted by Laclau and Mouffe (1985). I argue that this poststructuralist underpinning for discourse theory provides an explanation for how features of discourse such as misaligned expectations and competing versions of recovery may be understood as complexity generating factors within mental health service systems.

\subsection{Discourse and complexity}

In Chapter four, I argued that complexity was most manifest in the discursive practices, positionings and constructed meanings, identified through discourse analysis of talk spoken by individuals seeking to negotiate transforming mental health service systems and interfaces. Since complexity was felt most keenly within the subjective positioning and discursive practices of individuals, it makes sense to try to understand how discourse itself can generate complexity, since this would provide an underlying explanation for the complexity and fragmentation of services which has been identified in previous analysis.

An explicit theoretical exposition relating discourse theory to complexity is provided by Cilliers (1998) whose poststructuralist theory I employ as a key contribution to my argument in this chapter. This ties in with the poststructuralist view of discursive universalism held by Laclau and Mouffe (1985), introduced in section 3.7.1.2. Cilliers' (1998) main argument is that 
a poststructuralist understanding of language leads necessarily to a view of language ${ }^{19}$ as a complex system. In forming this argument, he first describes the development of poststructuralism out of structuralism, based on the critique provided by Jacques Derrida (1976). This critique centred on a refutation of Saussure's understanding of the relationship between the sign and the signifier (Belsey, 2002; Craib, 1992). Whilst this relationship is not fixed in structuralism, it is nevertheless assigned in a deterministic fashion by its place within the entire system or structure of language (Saussure, 1974). Cilliers (1998) notes therefore that the structuralist understanding of language is not a complex systems understanding. It is a closed system which may evolve over time, but "remains in a state near equilibrium" (Cilliers, 1998, p.42). Derrida provided a critique of the structuralist notion of language and meaning based on a refutation of what he referred to as the 'metaphysics of presence' (Derrida, 1976; 1978). This describes Derrida's accusation of a fundamental tendency in the whole tradition of Western philosophy, encapsulated in the central supposition of Saussurian theory that the meaning of a sign is present to the speaker when he uses it. Derrida rejects this principle of 'presence' in structuralist theory to propose the 'post' - structuralist principle that, instead of meaning being present in some way within the sign, meaning is determined by the 'différance' of the sign to the entire, linguistic system. This différance occurs not just in relation to the presently existing system of language but also to any potential system of signification which might be constructed by speakers in the future. Indeed, the unlimited potentialities of meaningmaking in language and discourse is accentuated by the emphasis in poststructuralism on 'parole' rather than 'langue' as the principal dimension within which new linguistic meaning is generated. ${ }^{20}$

\footnotetext{
${ }^{19}$ In this chapter, I will use the terms discourse and language interchangeably. In sections 3.3.2 and 3.6.4, I noted that discourse analysis focuses on the key role that language plays in the social construction of meaning (Burr, 2003). My theoretical perspective throughout this thesis has been to understand language as discourse in this way, within a social constructionist paradigm. Therefore, for the intents and purposes of the argument and theory developed in this chapter, discourse and language are understood as one and the same thing.

${ }^{20}$ In Saussurian structuralist theory, 'langue' is the structure and network of signs in language that give meaning to one another. 'Parole' is situated, concrete language use, which is ongoing with the passage of time as speakers construct new instances of language in talk and text in new and unlimited combinations. Whilst in structuralism meaning is generated in relation to 'langue', poststructuralist theory sees concrete language use or 'parole' as the principal dimension within which meaning is created, since the structure of language does not chiefly determine meaning.
} 
This poststructuralist understanding of language proposed by Derrida leads to an understanding of the linguistic system as a complex system, according to Paul Cilliers (1998). The poststructuralist system of discourse and language is an 'open' system and not a 'closed' one as with the structuralist conception. Meaning is not given in a determinant manner but rather on the basis of the unlimited potentialities of 'differance' in an unlimited system of new significations. Cilliers (1998) describes this state of affairs as follows:

"If language is closed off, if it is formalised into a stable system in which meaning is fixed, it will die, or was dead to start with. A living language is in a state far from equilibrium. It changes, it is in contact with other languages, it is abused and transformed."

(Cilliers, 1998, p.124).

A poststructuralist view of language and discourse is one where the meaning-making principle of 'différance' drives it constantly towards disequilibrium, in the manner of a complex system. The key driver for complexity, according to this view, is 'différance' since this corresponds to the greatest potential for functional differentiation in the entire system. Complex systems ontology is fundamentally characterised by the presence of the operation of functional differentiation of its component parts (Cillers, 1998; Luhmann, 1996; Osterberg, 2000). Consequently, disparate components operate in a manner which is independent of the whole system, generating complexity. Cilliers' (1998) view of différance within the linguistic system is that this source of meaning operates as a functionally differentiating and complicating factor. Meaning-making is determined in a manner unhinged and functionally differentiated from the entire system. This means that the system will be highly flexible and adaptive, and prone to unpredictability and disequilibrium (Cillers, 1998; Osterberg, 2000; Plesk and Greenhalgh, 2001; Rittel and Webber, 1973). Cilliers articulates this point as follows:

"The reflexivity of language - the loops in the network - is exemplified by the dynamics of différance. Language is constantly transformed by the way in which it is used."

(Cilliers, 1998, p.124)

In such a system, différance opens the way towards unlimited and escalating meaning-making potentialities, and there is the potential for generation of more possibilities than can be actualised, a central characteristic of complexity highlighted by Niklas Luhmann (1985). This theoretical understanding of language and discourse as a complex system forms the basis for 
me to perform theoretical generalisation in the next section, building my argument on the theme 'Misaligned expectations in negotiating transforming services'.

\subsection{Discourse complexity and misaligned expectations}

It is now possible to develop an understanding of misaligned expectations as a complex systems effect based on this theoretical understanding of discourse. It is important to consider that complexity will not be distributed evenly throughout the entire linguistic system ${ }^{21}$. Since complexity is generated in Cilliers' (1998) system by the meaning-making mechanism of différance, it will be at points of the greatest concentration of meaning-making activity that the greatest concentration of complexity generation will take place. Situations where participants express misaligned expectations of services in their talk will be such points of concentration of meaning-making activity and complexity, since individuals are attempting to make new sense of transforming service structures, with varying degrees of success of failure. From a discourse theoretical perspective, this sense-making activity should be regarded as a series of discursive events (which have been highlighted through previous analysis) as participants seek to comprehend and re-signify changing service experiences in their talk, and their positions in relation to these phenomena. For instance, analysis of James' talk in section 4.3.1.2, shows how he constructs a meaning for his experiences of involuntary transfer from secondary to primary services. This is achieved through the discursive device of topic-chaining (Gee, 2010) to construct a coherent sense of the gradual decline in secondary care provision. This is one indication that his talk involves concentrated meaning-making activity in the wake of transforming service structures. This constructed meaning also constitutes a misaligned expectation which was understood to be a complex systems effect in section 4.3.1.2, and all the more so here because such linguistic activity is understood to be complexity generating.

It is possible to generalise from this insight about complexity in discourse about misaligned expectations to view a variety of findings outlined in previous chapters as instances of

\footnotetext{
${ }^{21}$ Indeed, areas of discourse where a greater level of meaning-making activity occurs and therefore which are most associated with the meaning-making activity of différance will be the most complex areas within a generally complex linguistic system. These instances of discourse will be the most pronounced manifestations of complexity within the entire complex linguistic system.
} 
complicating discursive phenomena. The following figure shows a number of complex systems effects escalating throughout services, viewed through the lens of participants' experiences and the meaning-making activity of their talk. I argue that all of these effects can be understood to be particular foci of complexity within discourse, given the principle of différance as the basis for meaning-making:

\section{Figure 14: Complex service systems effects in Wales}

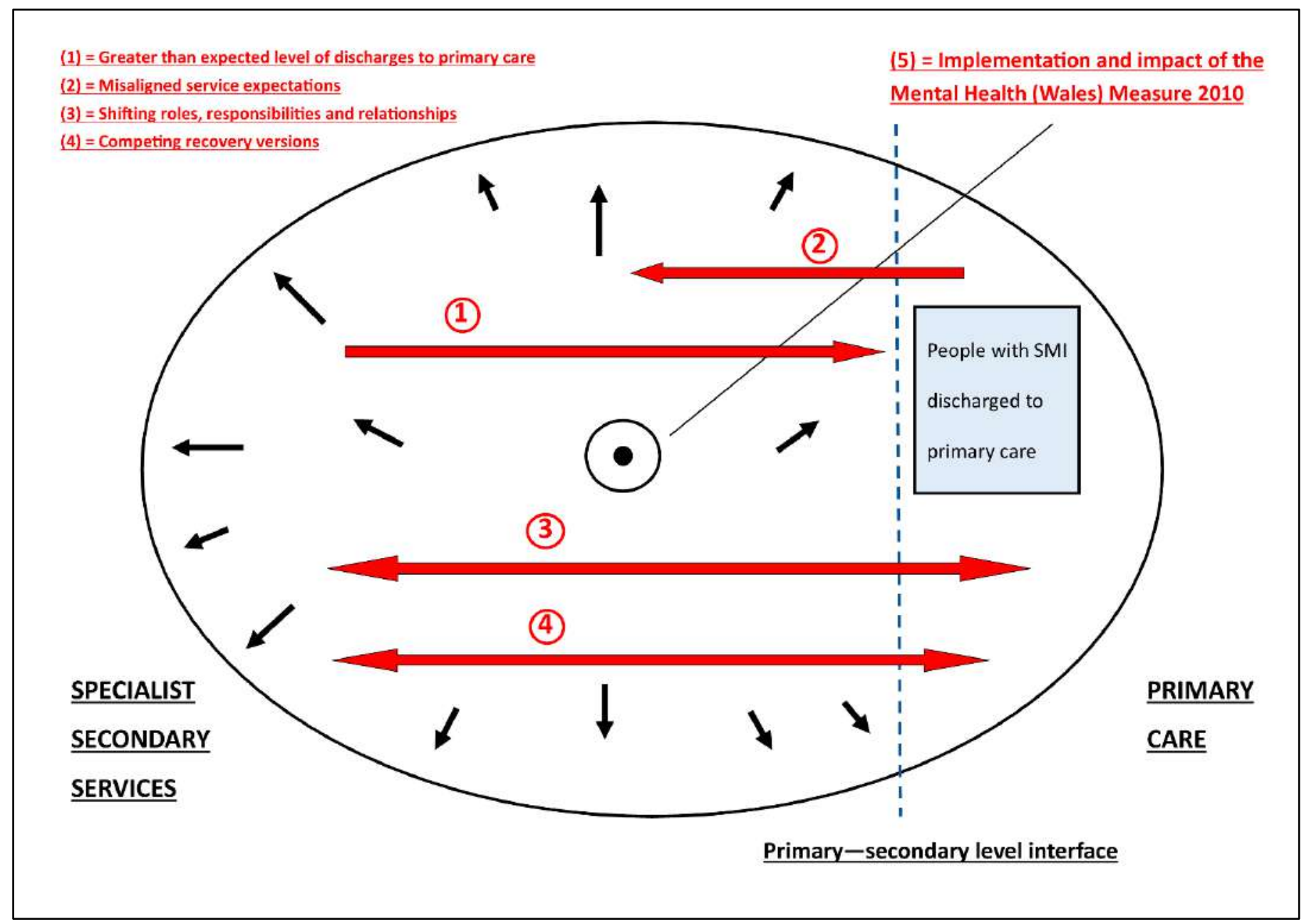

In this diagram, a number of complex systems effects, or waves of consequence, are illustrated within a complex service system. The first of these, marked numerical one, represents the phenomenon of a greater than expected level of discharges to primary care. The second wave of consequence, marked numerical two, represents misaligned expectations of transforming service structures and interfaces, which is being discussed in this section. To an extent this can 
be considered to be a further wave of consequence stemming from the greater than expected level of discharges, since Alex, James, Alun and Katy each operate from a position within primary care and its expanded role for mental healthcare delivery.

Both of these waves of consequence can be seen as manifestations of complexity within the social world, understood as discourse, according to the view of discursive universalism. As I have already argued, misaligned expectations of transforming services can be seen discursively as points of concentration of meaning-making activity and complexity as individuals negotiate transforming service interfaces. In section 2.5.2, I described a greater than expected level of discharges as a 'wave of consequence' (Rittel and Webber, 1973) stemming from implementation of the Mental Health (Wales) Measure. This latter effect may also be viewed discursively as complex in the light of the discursive universalism I adopted in section 3.7.1.2. If all features of the social world may be understood as discourse, then even more objective features of the world, such as a greater than expected level of discharges, must be seen as "constituted as an object of discourse" (Laclau and Mouffe, 1985, p.108). Since increased levels of discharges represents a zone of greater activity stemming from implementation of the Measure, this will also be an area of concentrated meaning-making activity surrounding the activity, and therefore also of complexity. For this reason, it is represented in the above figure as a complex systems effect.

Further waves of consequence are illustrated within the above diagram of mental health service systems in Wales. The phenomenon of shifting professional, voluntary or service user roles, responsibilities and relationships is marked numerical three, an effect described by Hannigan and Coffey (2011) as resulting from changing service structures and professional boundary lines. One key example of this found within the data set is the increased role for GPs as mental healthcare practitioners. This is a new role that it appears Alex struggles with from analysis of his talk in section 4.3.1.1. My analysis here focused not so much on the validity of his criticisms of the quality of secondary services, or proposals to solve issues related to a lack of a "longterm plan" (line 27), but on how Alex's complaint expresses increased tensions between professional groups within primary and secondary sectors. This then is an example of how the wave of consequence of more complex roles and interrelations between professional groups is 
expressed discursively as increased complexity-generating meaning-making activity, which takes the form of a complaint for Alex and other participants.

Similarly, I view Katy's misaligned expectations as expression in talk of her struggle to come to terms with an expanded role of self-determination under the Mental Health (Wales) Measure 2010, a policy seeking to re-orientate services and individuals towards a self-management recovery approach. This can be viewed as another instance of changing roles and relationships between individuals (Hannigan and Coffey, 2011; Plsek and Greenhalgh, 2001; see complex systems effect three in Figure 14), this time between professionals and service users. As with the wave of consequence marked numerical four, the arrow representing this complex system effect in the diagram points in both directions across the primary-secondary interface. This denotes the way in which this phenomenon, of which there are multiple manifestations (new GP roles, greater responsibility for service users and so on), generates escalating complexity in multiple directions in the service system, most particularly in both directions across the primary-secondary interface.

Competing versions of recovery are also represented by the two-way arrow marked numerical four in the above diagram. Although I shall argue on the basis of a related line of theory, based on the concept of floating signifier (Laclau and Mouffe, 1985), how competing versions of recovery are a complex effect, this phenomenon can be placed in the above diagram on the basis of theory developed so far in this chapter. Firstly, in the light of Plsek and Greenhalgh's (2001) view of health services as complex adaptive systems, the variety of recovery approaches talked about by participants can be seen as 'recovery technologies' acting as drivers for increased complexity (Smith-Merry et al., 2011; Woods et al., 2019). Secondly, the dissemination of 'recovery technologies' can be seen as the "various kinds of techniques, practices and instruments that embody and instantiate the values of recovery" (Smith-Merry et al., 2011, p.2). These are all meaning-making activities and will, through the meaning-making principle of différance, stimulate greater functional differentiation and complexity throughout an increasingly complex service system. 
The figure also illustrates the impact of the Mental Health (Wales) Measure 2010 as a catalyst for systemic complexity. Because the Measure attempts to implement recovery-based services, and at a simpler level because it instigates major change in the behaviour of services, such as increased levels of discharges and further effects stemming from this, I regard the Measure as a catalyst for complexity within the system. It is therefore represented in the above figure, marked numerical five. This piece of legislation can also be regarded as a discursive phenomenon within Laclau and Mouffe's (1985) view of the social world where all phenomena are elements of discourse. In support of this, Fairclough (2003) argues that legislation and policy may be seen as forms of discourse since a particular world-view and set of values are promoted through their language. In the case of the Mental Health (Wales) Measure 2010, this set of values is drawn from the predominant paradigm of recovery, of which it is a product (Bracken et al., 2012; Braslow, 2013; Morrow, 2013; Welsh Government, 2014c; 2015; Woods et al., 2019).

The remaining smaller and randomly distributed arrows represent in a general way the conditions of escalating complexity permeating Welsh mental health services. This may be due to the overall effect of the five specific complex systems effects identified in the figure, or due to other complicating factors such as the intrinsic complexity of mental illness, healthcare services and recovery (Bracken and Thomas, 2001; Bracken et al., 2012; Hannigan and Coffey, 2011; Pilgrim, 2008; Stanghellini, 2013). These background conditions may also be exacerbated by the various factors specifically identified in the above diagram.

An overall picture of escalating complexity in the form of a variety of objectively or subjectively-oriented discursive phenomena is therefore depicted in Figure 14. At this point of my argument, it may be objected that introducing the poststructuralist and complexity theories of Cilliers (1998) and Laclau and Mouffe (1985) adds little to the understanding already established in Chapter four that misaligned expectations are a complex system effect. However, this objection can be countered by noting that analysis within the original findings chapter merely asserts these phenomena to be complex systems effects in the light of theory provided by Rittel and Webber (1973) and Hannigan and Coffey (2011). These theories do not incorporate discourse theory and so provide no explanation for how complexity effects can be understood discursively, and therefore on the basis of the qualitative evidence produced by data 
analysis in this thesis. The explanation provided in this section, and developed in this thesis, provides a more extensive theoretical underpinning and route for theoretical generalisation grounded in the data set of this inquiry, given that the principal mode of inquiry within this piece of research is discourse analysis. A complex systems, poststructuralist theory of language and discourse thus provides a more fundamental and evidence-based explanation of findings which harmonises theoretically with the methodological approach of discourse analysis employed for this inquiry. The coherence of this explanation in my opinion better fulfils the requirement for cogency of theoretical generalisation (Mitchell, 1983). ${ }^{22}$

Having laid the foundation of poststructuralist complexity theory in order to provide an explanation of the complex systems effect of phenomena such as misaligned expectations of services, I now continue to develop my argument in the next section, in relation to the main finding of Chapter five described by the theme 'Competing versions of recovery'.

\subsection{Recovery as a floating signifier generating systemic complexity}

In Chapter five, I introduced the concept of 'floating signifier' provided by Laclau and Mouffe (1985) in order to advance my analysis of discursive behaviour among participants who construct a variety of recovery versions. I proposed that the polyvalent concept of recovery (Pilgrim, 2008) was a floating signifier, whose meaning has not been fixed by a particular discourse or agent. Consequently, I argue that other discourses are engaged in a process of continual discursive struggle to fix and appropriate the recovery concept within their order of discourse (Fairclough, 1995; 1998; Jørgensen and Phillips, 2002). Laclau and Mouffe’s (1985) poststructuralist view of discourse, which is the basis for their concept of floating signifier,

\footnotetext{
${ }^{22}$ It may also be objected that it is only by my introducing a poststructuralist discourse theory that the ingredient of complexity has been introduced into the understanding of findings. According to this view, complexity is not a feature of research phenomena but an overlay which has been introduced by application of this theoretical perspective. I would counter by falling back on the idea introduced in section 3.7.1 that arguments of theoretical generalisation have broad applicability because they characterise the ontological fabric of that part of the social world being investigated. The potential for application of a particular theory to particular set of social phenomena is not therefore a contingent and entirely flexible affair. It is not the case, in my view, that any theory can be fitted to any situation. Rather, the appropriateness and cogency of a particular theoretical application is illustrative of underlying ontological features of that part of the social world being investigated. This is consistent with the underpinning of contextualist constructionism I laid out in section 3.3.4, where potential theoretical interpretations are not boundless, but are limited by the propensities of underlying intransitive structures (Houston, 2001; Willig, 1999).
} 
accords with Cilliers' (1998) view of the instability of language and meaning. Jørgensen and Phillips (2002) summarise this position:

"Ernesto Laclau and Chantal Mouffe's discourse theory... is the 'purest' poststructuralist theory in our selection. The theory has its starting point in the poststructuralist idea that discourse constructs the social world in meaning, and that, owing to the fundamental instability of language, meaning can never be permanently fixed. No discourse is a closed entity: it is, rather, constantly being transformed through contact with other discourses. So a keyword of the theory is discursive struggle. Different discourses - each of them representing particular ways of talking about and understanding the social world - are engaged in a constant struggle with one other to achieve hegemony, that is, to fix the meanings of language in their own way. Hegemony, then, can provisionally be understood as the dominance of one particular perspective."

(Jørgensen and Phillips, 2002, p.6)

Within this scheme, the order of discourse (Fairclough, 1995; 1998) is understood as constituted by a continual discursive struggle of discourses attempting to fix the meaning of language in their own particular way. When such a linguistic fixing becomes dominant in Laclau and Mouffe's (1985) system, this is referred to as 'hegemony', according to their specific interpretation of the term ${ }^{23}$. Floating signifiers are signs which are particularly open to fixing, or hegemony, since they are cases where their meaning is particularly fluid or 'polyvalent', as is the case for the recovery concept (Pilgrim, 2008).

Laclau and Mouffe (1985) applied the concept of hegemony in a radical and extensive way as an integral component within their poststructuralist discourse theory. Since according to poststructuralism, no meaning can be fixed, with signs in a perpetual state of discursive struggle, hegemony is employed as an appropriate concept to embody the fixing of meaning.

\footnotetext{
23 The concept of hegemony, though used by Lenin, was developed into a powerful socio-analytical tool by Antonio Gramsci (1971). Gramsci was a leading light within the Frankfurt school of critical theory who proposed that, in addition to physical coercion, dominant power may be exercised by limiting the boundaries of a populace's socio-psychological horizons (Gramsci, 1971; Kincheloe and McLaren, 1994). In delimiting these sociological and psychological horizons, people's common-sense may be enmeshed with a particular dominant ideology (Bullock and Trombley, 1999; Abercrombie and Turner, 1978). Through this means, their consent is established without the more traditional recourse to violence or coercive force (Crossley, 2005; Kinchelow and McLaren 1994). The concept of hegemony is therefore a key concept within recent critical and emancipatory theory because it articulates the way in which popular consciousness may be subverted by simulating its inception of a dominant ideology. The quality of compliance and oppression thus produced is much greater than that which might be achieved by violence or coercion because the people believe that they themselves are the authors of the dominant ideology, infused into their consciousness by the process of hegemony (Barrett, 1991; Gramsci, 1971).
} 
Hegemony is seen as the governing principle of the linguistic system, and discourses are attempting to fix parts of the linguistic system in order to achieve a particular instance of hegemony. ${ }^{24}$

Returning now to the idea that recovery is a floating signifier, since the meaning of recovery is polyvalent and contested, it will be a focal point for this activity of discursive struggle, with a variety of competing versions or discourses seeking to fix its meaning. In other words, the floating signifier of recovery is a focal point for discourses seeking to hegemonize the floating signifier according to their own agenda or particular ideological stance. Since hegemony is a focal point for meaning-making activity, in the light of Cilliers' (1998) theory relating différance to complexity, it will also be a focal point for complexity generation. Once it is understood that recovery as a floating signifier may generate systemic complexity in this way, it can be seen why a proliferation of competing recovery versions will be a factor behind escalating service complexity.

This provides initial support for my argument in this discussion chapter that there are a proliferation of recovery versions which may be seen as avenues for discursive and systemic complexity. Returning to the data and my analysis of it in previous chapters in order to support this point, in Chapter five I identified numerous versions of recovery in participants' talk, many of which were competing. A number of recovery approaches $(n=5)$ were identified in conceptual analysis represented in Figure 7, as well as the 'Recovery' approaches' concept

\footnotetext{
${ }^{24}$ The universal application of hegemony within Laclau and Mouffe's discourse theory is reflected by its diverse and wide-ranging political applicability, which forms the basis for radical democracy (Epstein, 1996). This was achieved by broadening socialist political thinking beyond the traditional Marxist ground of explanation in the economic base. Laclau and Mouffe (1985) extended the basis of socialism to any situation of meaning-fixing within a language, understood poststructurally. Their work is a seminal piece amongst post-Marxist thought because it radicalises the emancipatory agenda of socialism to a much broader and more diverse span by incorporating poststructuralism into Marxism.
}

This poststructuralist conception of socialism as radical democracy rests upon an understanding of politics in which every position is constructed and negotiated anew, rather than being encumbered by an essentialist grounding in economic materialism (Epstein, 1996). The shift from traditional Marxism to post-Marxism championed the new social movements (Crossley, 2003; Habermas, 1984b), such as environmentalists, feminists, pacifists and, in mental health, user / survivor protest groups, as successors to earlier workers' movements in rekindling the emancipatory project of critical theorists. Laclau and Mouffe grafted poststructuralism onto Marxism making it relevant to the broader domain of cultural politics, that is arguably no longer responsive to classical Marxist theory (Maund, 2012). This involves a rejection of class politics and the old labour movements in favour of cultural politics promoted by the new social movements. 
under the category 'Third sector care and support' in Figure 8. These included holistic notions of recovery, focusing on wellness and the person rather than on illness and deficits, different recovery approaches and plans such as the recovery STAR (McKeith and Burns, 2010), exercise, and creative activity involving arts and crafts. I have also summarised various recovery versions above in section 7.2 whose construction can be related to instances of colonisation of the recovery concept in participants' talk. All of these instances can be seen as competing versions of the floating signifier of recovery which in the worldview of Laclau and Mouffe (1985) is the activity of discursive struggle generating escalating complexity.

More fundamentally, I would argue on the basis of analysis and theory here that it is an intrinsic quality of recovery to have the capacity to be discursively appropriated in a proliferation of versions with the potential to escalate complexity. Consequently, attempts to engender recovery-based services, or increased recovery-oriented activity of any form, will have the potential to introduce escalating complexity into services. This is an important consideration for attempts at service engineering which seek to achieve this aim, such as the Mental Health (Wales) Measure, since escalating complexity may not be desirable given the implications it has for service fragmentation.

\subsection{Diverse, self-oriented recovery versions contributing to a complex service landscape}

The status of recovery as a floating signifier, subject to hegemony in the sense provided by Laclau and Mouffe (1985), can be related to the inherently self-oriented nature of recovery, with its emphasis on individual empowerment and self-sufficiency (Copeland, 2000; Davidson, 2005; McKeith and Burns 2010; Pilgrim and McCranie, 2013). The flexibility and unfixed nature of its significance makes it suitable for highly individualised discursive appropriations and interpretations of its meaning (Senker and Green, 2016). I regard these appropriations as types of low-level hegemony enacted by service users and workers at the ground-level as they seek to interpret paradigms, theories and policies of recovery (Lipsky, 1980).

In this context of discourse, the recovery appropriations may have "deeply personal" (Anthony, 1993, p.527) meanings for different people (Edgley et al., 2012), as they seek to fix the meaning 
of this signifier according to their own individual perspectives and agendas. This subjectivity has the potential to generate multiple, competing versions of recovery, rooted in personal experiences and agendas. Recovery is often defined precisely in terms of it being a self-oriented and personal approach to tackling mental illness (Anthony, 1993; Davidson, 2005; Edgley et al., 2012; Repper and Perkins, 2003), and a facilitation of individual resources of social capital through empowerment and increased self-efficacy (Amering and Schmolke, 2009; Bonney and Stickley, 2008; Leamy et al., 2011; Tew et al., 2011). Support for self-oriented versions of recovery has also been found in analysis of data in previous chapters. The second and third recovery versions listed in the findings summary in section 7.2, represent a neoliberalist policy agenda of self-sufficiency and responsibility, and a more emancipatory focus on selfmanagement removed from services respectively. Additionally, analysis of talk in Chapter five indicates that Dylan, Alun and Tony each promote their own versions of recovery based on self-interested agendas. Respectively, these participants' recovery versions are based on a selfmanagement approach, recovery conceived as returning to work, and a recovery version of psychotherapeutic support and social inclusion approaches promoted by his third sector daycentre. On similar lines, another service user participant, Ian, a person in his thirties who suffers from Post Traumatic Stress Disorder, describes how he has completely cut himself off from service support in order pursue his own approach to recovery:

224. Ian: I've discharged myself. I've finished. Everything

225. from here on in, I will sort out myself.

[Ian, Service user, Phase 1, Participant 16, lines 224-225]

This extreme version of a self-oriented approach has resulted from iatrogenic experiences of both medical and psychological interventions. He talks about an "appalling” (line 471) experience of being on antipsychotic medication:

471. Ian: It was awful. It was absolutely appalling.

472. Researcher: Yes.

473. Ian: You know, I'd get up, to just go to the shop, and I

474. couldn't, physically, open a front door. I couldn't walk out of

475. the house - unless I was going to work, and that was okay - I

476. couldn't leave the house at all, not for anything.

477. Researcher: Yes.

478. Ian: And when you've got - at the time we had, well, 
479. four children, and two of them were very young, well, it put an

480. awful lot of pressure on my wife as well, you know.

481. Researcher: Yes, yes, yes.

482. Ian: It wasn't good. And that went on for probably twelve

483. months.

[Ian, Service user, Phase 1, Participant 16, lines 471-483]

He recounts his experience of psychotherapeutic provision in similarly negative and iatrogenic terms elsewhere in the interview. Firstly, he was on a waiting list for eighteen months culminating in art therapy which was different to the CBT and $\mathrm{EMDR}^{25}$ he had initially been promised. The art therapy had an iatrogenic effect according to Ian since it stimulated recall of traumatic memories that were then not dealt with therapeutically in a way which he found at all satisfactory. He also struggled with this approach since he was not artistic. The result of these experiences is that he now pursues an approach which is avoidant of service intervention and based entirely on his own personal resources. In response to my question about what his experiences have taught him about self-management, he says:

608. Ian: Other than giving me an appreciation that

609. anything that's going to improve the situation must come from

610. me, because it's not going to come from anybody else,

611. nothing. It's done nothing at all.

[Ian, Service user, Phase 1, Participant 16, lines 608-611]

In a similar though more extreme way to Dylan, Ian's account is illustrative of a self-reliant approach to recovery rooted in a desire to be emancipated from coercive and iatrogenic services. Further self-oriented recovery versions have been identified in analysis in previous chapters. For instance, certain third sector participants constructed recovery versions which adhered to the practices of their organisations which may be overtly recovery-related (Hannah's talk on the Recovery Star in section 5.4.1), or simply a label applied to pre-existing support practices (Tony's psychotherapeutic recovery approach in section 5.3.1) and generalised notions of 'holistic' care adopted by the speaker (see Sheryl's account in section 6.4.1).

\footnotetext{
25 'EMDR' or 'Eye Movement Desensitisation and Reprocessing', a type of psychotherapy aimed at treating the symptoms of emotional distress and trauma.
} 
In sum, the status of recovery as floating signifier leads to its appropriation by a variety of individual interpretations and agendas. To an extent, this is a legitimate activity. Recovery was originally characterised by Anthony (1993), in his oft-quoted statement again provided here, as a highly individualised and personal process of finding new meaning and purpose in learning to live with mental illness:

"Recovery is described as a deeply personal, unique process of changing one's attitudes, values, feelings, goals, skills, and/or roles. It is a way of living a satisfying, hopeful, and contributing life even with limitations caused by illness. Recovery involves the development of new meaning and purpose in one's life as one grows beyond the catastrophic effects of mental illness."

(Anthony, 1993, p.527)

From a discourse analytical perspective, this process of finding new meaning is manifested in the various meanings which individuals ascribe to the floating signifier of recovery. This is testified to by the variety of self-oriented versions of recovery constructed in analysis of participants' discourse in this and previous chapters. Recovery should therefore be centrally understood as a person-centred meaning-making activity related to the interpretation the individual wishes to adopt for their approach to mental healthcare. Such self-oriented interpretations of recovery will be diverse, and potentially competing, understood as various attempts to fix the meaning of recovery within the ongoing discursive struggle constituting the complex mental health service system.

\subsection{Recovery trajectory}

So far, I have described in terms of discourse theory how misaligned expectations and competing versions of recovery contribute to complexity in service systems. This has implications for phenomena such as continuities of care. Since they are all part of the same discursive system, complexity in one area will have implications for increased complexity in another area. In a complex and fragmented service system, characterised by features such as misaligned expectations and competing recovery versions, there will be greater potential for disconnection and discontinuity of care coordination (Gilburt et al., 2014; Gilburt, 2015, Weaver et al., 2017). This link between the competing versions of Chapter five and experiences of care discontinuities identified chiefly in Chapter six can be bolstered by the introduction of my concept of a 'Recovery trajectory'. This concept expresses the diverse number of pathways, 
involving varying degrees of care continuity, which persons with mental illness may take as they progress in their recovery. The concept is based partly on Strauss's (1985) concept of 'illness trajectory', meaning the particular unfolding of illness for the individual in relation to the social context of work undertaken by carers and workers to provide treatment (Hannigan and Allen, 2013; Strauss, 1985). This includes the patient himself, within the recovery paradigm, since this encourages a self-management basis for care provision (Copeland, 2000; Davidson, 2005; McKeith and Burns 2010; Pilgrim and McCranie, 2013). The Recovery trajectory is also based on the sense of recovery as a personal journey (Pilgrim and McCranie, 2013). Here, recovery is understood as a "process of change through which individuals improve their health and wellness, live a self-directed life and strive to reach their full potential" (SAMHSA, 2011, cited in Pilgrim and McCranie, 2013, p.49). I consider this highly contextualised understanding of progress through illness to be analogous to Strauss's (1985) concept of illness trajectory which involves more than the illness itself, including also the social context of sickness as it develops over a period of time. Both of these concepts therefore contribute to the concept of Recovery trajectory. It should be noted that the concept is distinct from that of 'care pathway' (Allen, 2009; Håland et al., 2015; Panella et al., 2012; Schrijvers et al., 2012), which has the sense of being a more pre-determined and objective treatment route, and does not capture the contextual, hermeneutic and contingent conditions inherent within recovery approaches and mental illness (Bracken and Thomas, 2001; Bracken et al., 2012).

The concept of a Recovery trajectory allows a clearer connection to be made between analysis constructing recovery versions and more objective features of the social and discursive world such as discontinuities of care, barriers to care coordination and impediments to collaboration between the third sector and primary care. A direct connection can be made between a particular recovery version and a related care pathway which may or may not be continuous and well-coordinated. Care coordination will be more difficult when there is a great variety of disorganised recovery trajectories dispersed throughout a complex service system. This will impact negatively on care continuity, which is one of the principal aims of care coordination (Burns et al., 2009; Schultz and McDonald, 2014; Sweeney et al., 2012). Collaboration between different service sectors will also be impeded because different recovery trajectories may not be compatible, impacting negatively on cross-boundary continuity (Adair et al., 2005; Weaver et al., 2017). This, I would suggest, is what is occurring in the discourse of Tony, Hannah and 
Robert, where two third sector workers position their organisation's approaches in opposition to the nascent recovery approaches of the GP. The recovery trajectories articulated by participants within these different service sectors are based on recovery versions that are constructed in such a way that they are not compatible with one another. It could be said at this point that not all recovery versions and ensuing trajectories would necessarily be competing and that there could, for instance, be complementary versions, parallel or closely aligned versions. However, the weight of the evidence presented in data analysis within this thesis strongly implies the absence of such cohesion and the presence of disharmony between recovery versions. Since I have argued in this section that a recovery version can be understood as a Recovery trajectory, competing recovery versions can be seen as competing recovery trajectories which together will form a fragmented service landscape involving discontinuities of care. For example, conceptual analysis, which has been built upon by further discourse analysis, is weighted towards tensions between approaches of the third sector and that of primary care, and impediments to collaboration, rather than positive experiences of collaboration. Additionally, in Chapter six I showed that the weight of evidence supports the presence of care discontinuities rather than care continuities in various areas of services, experienced by participants, concentrated within primary care. Care continuities and scenarios of successful collaboration between service sectors do exist in analysis of participants' talk, as evidenced by concepts such as 'Positive experiences of collaboration' between the third sector and primary care (Figure 10), and the category 'Experiences of practices promoting continuities of primary care' (Figure 11), though these are not in the majority according to the evidence and analysis of this thesis. The issue then becomes how a greater tendency towards complementary versions, parallel and closely aligned trajectories might be instigated in a system of escalating complexity, a challenge which may be located in the arena of competing recovery versions.

The theory in this section based on the concept of Recovery trajectory may be added to the argument I have been building in this and previous chapters that service conditions where disparate recovery versions are generated will be increasingly complex and fragmented. Multiple recovery versions will lead to multiple recovery trajectories which make up a fragmented service landscape. I now represent this pictorially in the following figure: 
Figure 15: Competing recovery versions generating system complexity and service fragmentation

Mental Health (Wales) Measure 2010 implementation

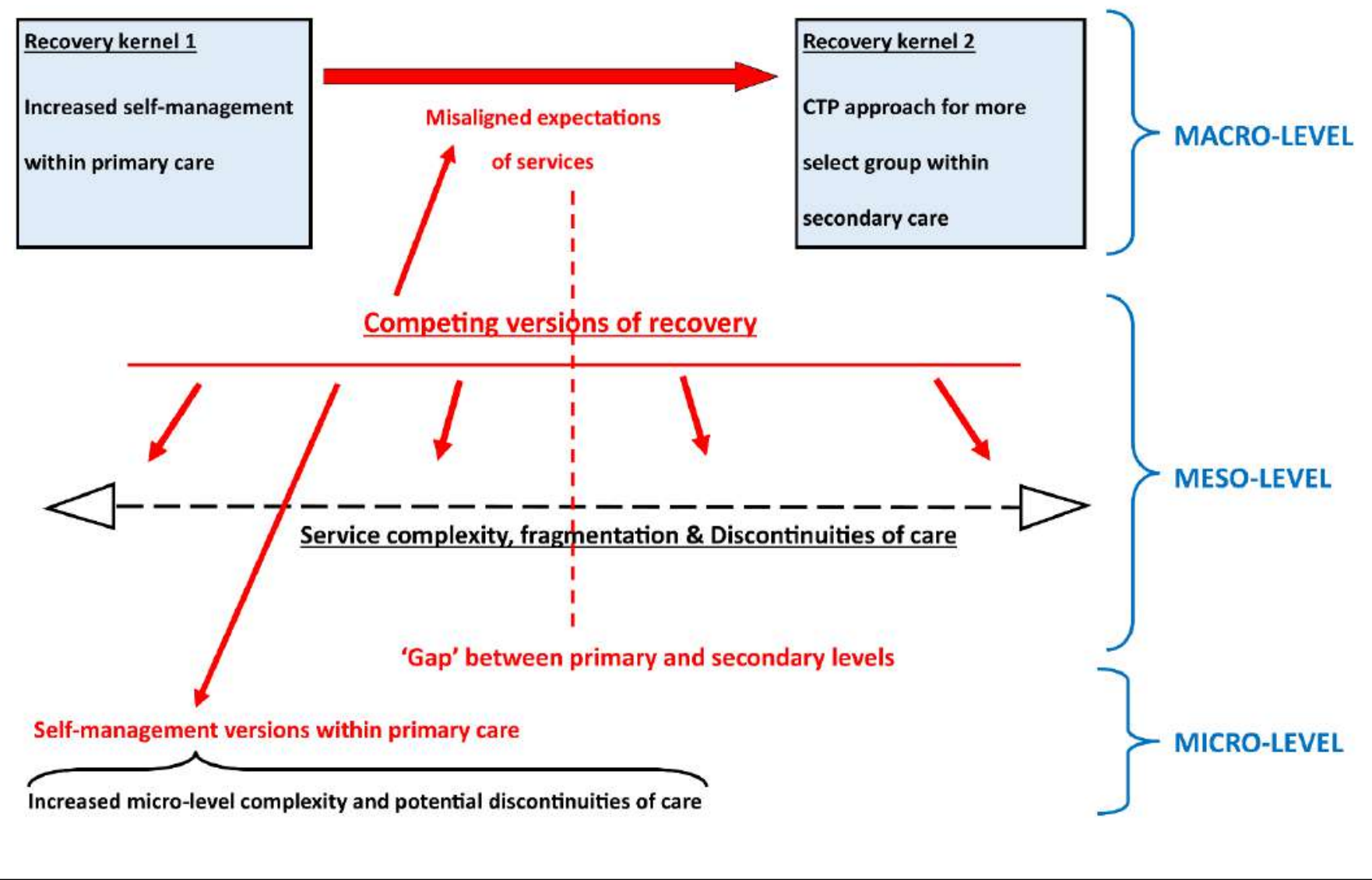

The complex landscape is represented on macro-, meso- and micro- levels, following the nested complex systems ontology provided by Byrne (1998) and applied by Hannigan (2013) to mental health service systems. Competing versions of recovery are primarily depicted at the meso-level, to indicate their central role and distribution across macro-, meso- and microlevels. The impact, via recovery trajectories and discourse, of competing versions of recovery is also shown primarily to occur at the meso-level, since this is where policy implementations of recovery, such as the Mental Health (Wales) Measure, and ground-level, self-oriented recovery versions may combine to impact upon care continuities and discontinuities. The Measure is therefore situated at the macro-level of policy and service strategizing (Hannigan, 2013). Here, as in the previous figure, it is depicted as a key catalyst for service complexity. 
As has been argued in section 6.3.3 and analysis of Maria's talk, the Measure overlies two recovery versions, or kernels, as they are labelled here. These are recovery as increased selfmanagement after discharge to primary care, and holistic and co-produced recovery under the now mandatory CTP approach within secondary care (Welsh Government, 2013; 2014c; 2016). They are considered kernels and not versions since they exist only theoretically in the literature accompanying implementation of the Measure. Actual versions of recovery will occur at mesoand micro-levels in multiple forms after implementation, as a personalised interpretations of these directives in the meaning-making or hermeneutic dimension of individuals' approaches to mental illness and recovery (Lipsky, 1980; Middleton, 2007; Stanghellini et al., 2013; see section 7.6 above).

Also represented in this figure is the gap between primary and secondary levels which I argue is exacerbated by implementation of these two recovery versions at the macro-level. This was also one of the findings of Chapter six in analysis of Maria's talk, and the concept constructed from participants' talk of a 'Gap' between primary and secondary service levels' (see Figure 6). Here, I argued that analysis of Maria's talk revealed these versions can be competing at the ground-level. This dual-pronged nature of the Measure's recovery implementation actually accentuates the gap between primary and secondary levels which is experienced as a care discontinuity by individuals who are discharged to primary care and lose their care coordinator.

The presence of this gap between service levels raises the concern that there may be a certain category of individuals who occupy the space between these two parts of the system and do not fit neatly into either level. Consequently, their expectations for good, continuous healthcare provision are not being met by a system which has been poorly prepared for them. This potentially feeds into the frustrated, misaligned expectations of participants whose talk was analysed in Chapter four, such as Alun, James and Katy. These findings reflect concerns raised in literature that people may be caught somewhere in the middle between primary and secondary services (Gofal, 2013; Gofal, 2014). Revisiting my own data, there is further evidence of this gap at the interface of primary and secondary levels from both service user and supporting worker data alike. Katy, whose talk was analysed in Chapter four, describes how she was discouraged from further help-seeking at the secondary level: 
37. Katy: You know? It made me feel like, "I don't

38. even want to go back there anymore for the help", because

39. they made me feel like I was just not ill enough.

[Katy, Service user, Phase 1, Participant 4, lines 37-39]

Supporting this perception, Luke, a third sector day centre manager in his thirties, interviewed in the second phase of data collection, describes how individuals may even be lost to services as they transit across the secondary-primary gap, especially if they are unaware of support which may be provided by the third sector:

371. Luke: I think if (.2)

372. there was more involvement from the third sector, "cause

373. what I'm finding is perhaps as I stated earlier that

374. individuals are perhaps not deemed unwell enough. That

375. is the worry then where if they're not deemed unwell

376. enough, whether then it's just solely back to the primary level

377. or if they're unaware of what other support networks are

378. around that you lose that individual.

[Luke, Third sector manager, Phase 2, Participant 10, lines 371-378]

A person being completely lost to services would represent a profound discontinuity of care and interruption of their Recovery trajectory.

This and the other structural features highlighted in this section and the accompanying figure contribute to my argument about escalating service system complexity, fragmentation and discontinuity under the impact of a proliferation of recovery versions at micro-, meso- and macro-levels. Analysis of participants' talk, and theoretical argument based on this, reveals that people are experiencing service provision as increasingly complex, fragmented, and difficult to navigate, leading to experiences of discontinuity. In the next section, I develop my argument further by placing this emerging picture into the context of a postpsychiatric view of contemporary mental health services.

\subsection{A postpsychiatric perspective on fragmented mental health services}

The integration and development of theoretical strands in the previous sections has contributed to what I argue is an emerging picture of Welsh mental health services that are complex, 
disconnected and fragmented. My analysis in previous sections has identified concentrations of meaning-making activity and recovery trajectories as the key conduits for this complexity. At a more fundamental level, mental health and illness can itself be considered to be a complex phenomenon rooted in the individual lived experience of the service user. This view of psychiatry, promoted by in particular by Karl Jaspers (1997 [1913]), which emphasises a person's existential orientation and phenomenological experience of mental illness, is seen as the basis for person-centred, recovery-oriented care in the schools of values-based practice (Stanghellini et al., 2013) and postpsychiatry (Bracken et al, 2012; Middleton, 2007). According to these schools of thought, the notion of mental illness is itself contested which has led to obfuscation of the debate surrounding mental health (Rogers and Pilgrim, 2014). Thomas Szasz, who was a leader of the antipsychiatry movement, questioned the objective reality of mental illness, describing the 'myth of mental illness' as the psychiatric construction of diagnostic labels out of everyday 'problems of living' (Szasz, 1974a). This critique has softened within recent decades as the antipsychiatry movement has given way to critical psychiatry (Double, 2006a; Double, 2006b; Double, 2009; Heaton, 2006; Ingleby, 2006). However, this subsequent movement retains the assertion that mental illness and recovery should be understood on a more complex, hermeneutic basis, and not just in terms of a viewpoint dominated by a reductionist, positivist biomedicine (Bracken and Thomas, 2001; Bracken et al., 2012; Jaspers, 1997 [1913]; Nettleton, 2013; Stanghellini, 2013). This corresponds to one of the arguments I have been developing in relation to recovery that it should be understood as a person-centred meaning-making activity related to the individual's interpretation of their approach to mental health and care. The critical psychiatry movement, and the related school of postpsychiatry, have promoted this notion that the inherent complexity of mental illness and recovery is rooted in the personal and subjective dimension within which mental illness is interpreted. This postpsychiatric perspective is summarised by Bracken and colleagues (2012):

"Good psychiatry involves active engagement with the complex nature of mental health problems, a healthy scepticism for biological reductionism, tolerance for the tangled nature of relationships and meanings and the ability to negotiate these issues in a way that empowers service users and their carers."

(Bracken et al., 2012, p.433) 
A more individualised, hermeneutic approach to mental illness and recovery has the potential to generate greater complexity in the social world, described here as a "tangled nature of relationships and meanings" (Bracken et al., 2012, p.433). In mental health services characterised by postpsychiatry, there is a greater need to negotiate complex, shifting service interfaces and to develop new approaches to tackling mental illness where service users have much more empowerment and responsibility in conducting this activity.

Poststructuralism, which has been the context of discourse theory in this chapter, is closely linked with postmodernism, the philosophical underpinning of postpsychiatry (Cilliers, 1998; Craib, 1992; Delanty, 2005; Lyotard, 1984). Indeed, poststructuralism may be viewed as the appropriate theoretical position on language and discourse within the broader field of postmodernism. Postmodernism itself is difficult to define, although it can be understood as a cultural movement situated in art, architecture and literary theory (Belsey, 2002; Cilliers, 1998; Craib, 1992). Regarded as the cultural condition of contemporary society, postmodernity is characterised by fragmentation and disorder (Craib, 1992; Fuat Firat and Schultz, 1997; Lyotard, 1984; Thompson and Troester, 2002). The analysis and theory which has been developed in this thesis points towards a postpsychiatric or postmodern view of fragmented and complex mental health service systems in Wales. At the same time, I have argued that recovery is the key paradigm for contemporary Welsh services, within which competing versions are generated. This accords with the literature identifying recovery as the dominant paradigm for contemporary mental health services in general (Bracken et al., 2012; Braslow, 2013; Morrow, 2013; Woods et al., 2019). There can therefore be seen to be a close relationship between the conditions of postpsychiatry, or postmodernity, and the paradigm of recovery in mental health. Both are related underlying cultural conditions in which complexity, fragmentation and discontinuity in services and care will be encouraged. Because of this close connection, I propose that recovery can be seen as the principal paradigm for mental health services which are situated in the sociological and cultural conditions of postpsychiatry. This means that the recovery paradigm has the decidedly postpsychiatric implications of service fragmentation. The service fragmentation which this implies is potentially problematic since fragmentation may engender care discontinuities, whilst continuous and coordinated care is a cornerstone of good mental health care delivery (Burns et al., 2009; Schultz and McDonald, 2014; Sweeney et al., 2012; Weaver et al., 2017). To an extent, this postpsychiatric connection 
between recovery and care discontinuity is counter-intuitive as one might expect activities and approaches which promote recovery should improve care continuity. However, the implication of my argument here, based on the evidence of this thesis, is that the relationship between recovery and care continuity is fraught, and that recovery appropriation has the potential to generate care discontinuities, as well as having the potential to facilitate better care and care continuities. However, at the present time there is little quantitative evidence to clarify the relationship between recovery approaches and care continuity dimensions. This thesis then provides initial, exploratory evidence for what I argue is a circuitous relationship between recovery and care continuity, an understanding of which could be developed further by research building on my findings and conclusions (see section 8.4.4).

It may be commented that the recovery paradigm, occupying a postpsychiatric cultural context, represents an attempt to move beyond a modernist framework for mental healthcare towards a new sphere in which service users are emancipated and empowered (Bracken and Thomas, 2001; Bracken et al., 2012; Middleton, 2007). This may involve progress in resolving older problems of institutionalisation, psychopharmaceuticals, positivist medicalisation and authoritarian psychiatry (Braslow, 2013; Edgley, 2012; Gilburt et al., 2014; Moncrieff, 2006; 2009). ${ }^{26}$ However, one of the overriding arguments of this thesis has been that the novel cultural conditions of recovery may also lead to new problems and issues. Progress has arguably been achieved by engaging with the hermeneutic nature of mental health problems, where there may be greater consideration of complex contexts and social determinants of mental illness (Bracken and Thomas, 2001; WHO, 2014). However, the issue is now that this progression, though more true to the lived experiences and complex hermeneutic context of service users, has generated a complexity and fragmentation in services that is potentially counterproductive, especially when this manifests in the form of discontinuities of care. Partly, this is testified to in the various experiences of care discontinuities and impediments to collaboration which have featured in findings. Gilburt and colleagues (2014) describe this state of affairs succinctly:

\footnotetext{
${ }^{26}$ This statement should to an extent be qualified by critiques concerning whether deinstitutionalisation has really meant deinstitutionalisation or if there has merely been a shift of institutional care from hospitals to a constellation of revolving-door dwellings and ghettos such as prisons, third sector day centres, and board and care homes (Braslow, 2013; Mind, 2008).
} 
"The development of services focused on specific groups of individuals or needs has created a plethora of complex pathways for individuals to navigate in order to access services."

(Gilburt et al., 2014, p.13)

In other words, the cultural shift, most recently under the banner of recovery, towards emancipation of service users and a prioritisation of person-centred, individualised needs and perspectives, has simultaneously formed the conditions for escalating service system complexity. Fragmentation of services is one of the potential results of this, which would be in keeping with the cultural characteristics of postmodernity, or its surrogate within mental health of postpsychiatry. Thus, new difficulties have surfaced surrounding issues of how to successfully navigate and negotiate shifting service interfaces, how to tackle barriers to producing coordinated care, and the difficulties and impediments facing good collaboration between service sectors. Many of these difficulties have been reflected in experiences and analysis of talk about difficulties participants have faced in negotiating systems interfaces and working together.

Arguably, an emancipatory project for mental health hinging on recovery has achieved certain goals whilst new difficulties engendered by this paradigmatic shift have not been adequately addressed. A central issue appears to be that the advancement of personalised recovery approaches has led to a problem often associated with emancipatory paradigms, which is the difficulty of how autonomous individuals can live and co-operate with one another without this leading to conflict and disorder (Heywood, 2007; Tucker and Swartz, 1926). A further, related problem, which I will deal with in the next section, lies in the predominant neoliberalist forces behind colonisation of the recovery concept in the form of top-down, policy programmes seeking to implement recovery-based services. As part of the process of laying the groundwork for tackling these issues, I now introduce a final theoretical layer of Habermasian philosophy.

\subsection{Systemic neoliberalist recovery versions versus lifeworld recovery versions}

Consideration of Habermas' philosophy contrasting strategic and communicative action, or system versus lifeworld (Habermas, 1984a; 1984b), allows me to incorporate a further perspective from critical sociological theory into this ongoing discussion (Crossley, 2005; Craib, 1992; Kincheloe and McClaren, 1994; Porter, 1998). This perspective has the potential 
to expand upon the idea of colonisation of the recovery concept, which has been one of the major findings of this thesis, and to be a key driver for competing versions. I shall now outline his social theory of colonisation of the lifeworld by firstly describing Habermas' distinction between system and lifeworld.

The central idea underlying the distinction between system and lifeworld is that a fundamental division has occurred in modern society between a systemic sphere and the domain of the lifeworld (Crossley, 2005). The concept of the lifeworld is developed from Alfred Schutz (Schutz and Luckmann, 1974) and Gadamer (1989 [1975]; Grondin, 2003), and can be defined as the context-forming horizon of social action and consciousness, the site of symbolic or communicative interaction which occurs between individuals on the basis of mutual, prereflective understandings, norms and values (Crossley, 2005; Pusey, 2002):

"In phenomenological terms the lifeworld comprises that vast stock of taken-for-granted definitions and understandings of the world that give coherence and direction to our everyday actions and interactions."

(Pusey, 2002, p.58)

The appropriate form of analysis for this domain of symbolic interaction is therefore hermeneutic. Indeed, Habermas employs Gadamer's (1989 [1975]) hermeneutic principle of a 'fusion of horizons' to describe the mutual understanding which is achieved between individuals as they carry out communicative interaction at the level of the lifeworld (Pusey, 2002).

In contrast with the domain of the lifeworld is the systemic sphere, which has arisen due to an 'uncoupling' of certain sectors of the social world from the lifeworld, primarily the economy and state (Pusey, 2002). These areas of modern society can no longer be grasped at the hermeneutic level of symbolic interaction within the lifeworld. They have become so complex that they can no longer be comprehended at the level of everyday understanding (Crossley, 2003; McCarthy, 1984; Porter, 1998). Functional rather than hermeneutic analysis is therefore appropriate for the systemic sphere (Crossley, 2005). 
The notion of the colonisation of the lifeworld can now be introduced in terms of this fundamental ontological dichotomy within Habermas' thought. Colonisation of the lifeworld is based on the idea that the systemic sphere, having been uncoupled from the lifeworld, then threatens to re-enter and dominate the domain of the lifeworld, invading or colonising it "in the same manner as colonial overlords, displacing and intruding into areas of the lifeworld that were formerly 'free' of them" (Layder, 2005, p.173). In his introduction to his translation of Habermas' 'Theory of Communicative Action', McCarthy (1984) describes this process of colonisation of the lifeworld in terms of the capitalist economy as follows:

"In this way, the increasingly complex economic-political system penetrates ever deeper into the symbolic reproduction of the lifeworld, drawing ever-new spheres of communicatively structured interaction into the vortex of capitalist growth" (McCarthy, 1984, p.XXXViii)

This process of colonisation is 'pathological' (Crossley, 2005; Layder, 2005; Pusey, 2002) because the invasion of the lifeworld undermines the possibility of symbolic and communicative action. The systemic sphere of the market precisely is functional in its mode of comprehension, rather than hermeneutic, and it is this functionalist mode of meaning which is being introduced forcibly into the domain of the lifeworld so that it becomes distorted:

"The internal systemic logic of capitalism can be rendered in social-theoretical terms by the formula that the functional necessities of systemically integrated domains of action shall be met, if need be, even at the cost of technicizing the lifeworld."

(Habermas, 1984b, p.345)

System elements become inscribed in the domains of everyday life so that the lifeworld becomes increasingly divorced from its inherent meanings and normative values. Colonisation of the lifeworld therefore gives rise to "systematically distorted communication" (Habermas, 1984b, p.388) which undermines its meaningfulness and authenticity (Crossley, 2005).

The message of Habermas' version of critical social theory can be seen as a continuation of the Frankfurt school's message that the emancipatory potential of reason has been eroded by the progressive colonisation of reason by the economy and the state (Chouliaraki and Fairclough, 1999). Habermas' message is therefore an emancipatory one, compelling people to defend the 
sphere of the lifeworld against pathological and distortive encroachments from the systemic sphere. Michael Pusey (2002) states this state of affairs as follows:

"Basically Habermas invites us to look at our own modern condition as a kind of tug-of-war between the lifeworld and the system. Communicative rationality can only arise in the lifeworld as an achievement of communicative reason that might, eventually, lead to more rational structures .... Every painful step in this direction involves a linguistification of reified ${ }^{27}$ (naturelike) system structures that have to be re-appropriated through communicative action into the lifeworld. Given the unyielding organizational principle of capitalist society, the lifeworld is, from the other direction, constantly subjugated to mediatized colonization by the economy and the state (money and market, power and bureaucracy, respectively)."

(Pusey, 2002, p.107)

A major instance of the systemic sphere attempting to colonise the lifeworld identified by Habermas is the economic system of capitalism (Crossley, 2003; Habermas, 1984a; Habermas, 1984b; McCarthy, 1984). This is a highly appropriate theoretical application within this discussion about recovery experiences and service complexity. Recalling the summary of colonised recovery versions listed in section 7.2, the first two major types appropriation can be seen as instances of neoliberalist hegemony and colonisation, in the light of theory presented in this and previous sections. Focusing on the first of these, colonisation by a neoliberal policy agenda of work-as-recovery, a number of participants $(n=4)$ constructed recovery as being characterised by a return to work (see concept 'Productive and occupying work' under category 'Recovery approaches and constructions' in Figure 7). I analysed Alun's talk constructing this recovery version in section 5.2.3. Another instance of this in participants' talk is found with Patricia, a female service user in her forties who suffers from depression. When I asked her what her idea of recovery was, she stated emphatically that this was "Getting back to work" (line 589). In discussing this, she describes at one point the sense of pressure she feels she is under to return to work:

789. Patricia: You know I want to get

790. back to work, the government says they want me off

\footnotetext{
${ }^{27}$ Reification may be defined as "the apprehension of the products of human activity as if they were something other than human products - such as facts of nature" (Berger and Luckman, 1991, p.106). Here, entities produced in the communicative sphere have been reified by system elements, so that they have become dislocated from the lifeworld. In this case, "the dialectic between man, the producer, and his products is lost to consciousness. The reified world is, by definition, a dehumanised world" (Berger and Luckman, 1991, p.106).
} 
791. benefits and back to work. I want to be off benefits

792. because they do my box in. You know, the nagging, the

793. performing - you've got to fill out reams and reams of

794. forms, which stress me out to the point of no return. I

795. would rather go to work and clock in and clock out. But I

796. don't know what to do with the symptoms that I have. I

797. don't know how to deal with them. What do I do?

[Patricia, Service user, Phase 1, Participant 2, lines 789-797]

Her talk refers to the bureaucratic pressure of "nagging", (line 792) and the stress of having to "fill out reams and reams of forms" (lines 793 to 794). Indeed, her statement that the government wants her off benefits and back to work (lines 790 to 791) indicates this outside pressure as a key factor behind her version of recovery-as-work. Her alienation from this agenda is expressed in her dramatisation (Goffman, 1959) of work-seeking as an activity she has to "perform" (line 793) in order to satisfy the requirements of authorities. This is not to say that her desire to work is not to an extent a genuine and valid one, but rather that a great deal of the impetus for returning to work comes from an external source. Her understanding of recovery-as-work could also be said to accord with a very narrow interpretation, or even a misinterpretation, of the recovery model (Ramanuj et al., 2015; Recovery in the Bin, 2019). That Patricia has this narrow interpretation may be due a greater variety of recovery approaches not having been explained to her. This could be seen as another way in which the systemic sphere has invaded Patricia's lifeworld and social environment, since it has squeezed out the potential opportunity for more authentic recovery interpretations to be discussed or understood. In this way, a neoliberalist agenda supporting work-as-recovery can be seen to be responsible for a colonisation and systemic distortion of Patricia's way of understanding or communicating with others about potential recovery versions. This has limited her own unencumbered potential to engage in the meaning-making process of recovery construction.

Further support for the notion that work-as-recovery is a particularly hegemonic or colonised version may be found in the literature. Finding meaningful work does feature as an element within some theories and approaches for recovery. For instance, the Recovery Star includes work as one of its ten areas of life development (McKeith and Burns, 2010). The measurement scale, 'ReQoL', includes 'daily activity' as one of its key components which could include vocational occupation (Connell et al., 2012). However, in their review of $n=97$ papers, Leamy 
and colleagues (2011) find only one publication which promotes work as a major element of recovery, by Provencher and colleagues (2002). Finding meaningful work therefore occupies a limited place within the broad body of literature on recovery. Against this there is the burgeoning literature critiquing a neoliberal colonisation of recovery where the concept is interpreted in a very narrow way as getting people out of a state of dependency on benefits and services, and back into employment (Becker et al., 2010; Mind, 2008; Ramanuj et al., 2015). The literature asserts that this narrow recovery version is influenced by the introduction of external policy agendas governed by neoliberal principles where the central goal is to reduce dependency on services and welfare (Braslow, 2014; Mind, 2008; Morrow, 2013; Ramanuj et al., 2015; Recovery in the Bin, 2019). This has been described by Friedli and Stearn (2015) as 'psychocompulsion' referring to ways in which mental health services are engineered to reduce the number of benefit claimants, and lower the level of dependency on health services.

In addition to this, the second recovery version listed in section 7.2 is indicative of how an emphasis on self-sufficiency and responsibility may be the result of colonisation by a neoliberal policy agenda underscored by these pursuits. A number of commentators have observed that there is a strong neoliberalist ideological theme of self-sufficiency and self-orientation running through the value system that underpins the concept of recovery (Braslow, 2013; Copeland, 2000; Davidson, 2005; Edgley et al., 2012; Morrow, 2013; Pilgrim and McCranie, 2013; Woods et al., 2019). Recovery in this sense is underpinned by an ethos and expectation of selfmanagement and increased client responsibility in the context of neoliberalist cuts to healthcare funding, and welfare benefits (Morrow, 2013; Pilgrim and McCranie, 2013; Thomas, 2016). The Mental Health (Wales) Measure 2010 may regarded as one such instance of policy because of its emphasis on self-sufficiency within the context of primary care (Lester and Gask 2006; Pilgrim and McCranie, 2013; Ramanuj et al., 2015). Indeed, its dependence upon prudent healthcare principles can be seen as a neoliberalist influence. The Measure can therefore be firmly situated within the neoliberal consensus of certain policy-makers on recovery, and can be seen as an instance of this kind of colonised neoliberalist recovery version (Department of Health and Human Sciences, 2003; DoH, 2009; SAMHSA, 2004). Arguably, the paucity of this narrow, neoliberalist recovery version based on self-management after discharge to primary care is manifested in the limitations on healthcare access, care coordination and care continuity identified in analysis of Sheryl and Bernadette's talk in section 6.4. 
A degree of controversy surrounds whether recovery is intrinsically neoliberalist, especially when one considers that recovery was born out of an era when neoliberalism was the dominant ideology (Gamble, 1994; Palley, 2005). Braslow (2013) views recovery as inherently neoliberalist, being originally characterised by Reaganite neoliberalism in the United States, where the movement originated in the 1980s:

"Recovery as a guiding principle nests neatly within this broader context of neoliberalism and state policies of the past 25 years.... Reagan's theory of poverty is identical to the recovery theory of illness and chronicity except that instead of the public welfare system, the public mental health care system is to blame."

(Braslow, 2013, pp.800-801)

Braslow (2013) goes on to argue that embedded within the recovery vision are the ideological values of neoliberalism, which are concerned with increasing individual responsibility and simultaneously cutting back dependency on welfare. Such versions are predominantly promoted in the form of policy, of which I consider the Mental Health (Wales) Measure 2010 to be a local example. Braslow (2013) notes that "Neoliberalism has become hegemonic as a mode of discourse" (Harvey, 2005, p.3, cited in Braslow, 2013) and asserts not so much that the recovery concept has been hegemonized or colonised by neoliberalism but that recovery is itself the form which neoliberalist hegemony takes within the mental health field.

In parallel with this view, Edgley (2012) points out that the neoliberalist cultural context was vital for the conception of recovery, and that this is a key factor behind the ease with which it is appropriated in policy-frameworks:

"It is in the political context of the growth of a neo-liberal consensus that the Recovery paradigm has developed, to the point which it has become enshrined in mainstream mental health policy frameworks.... The Recovery paradigm has achieved this status because it appears to chime with the new consensus, particularly in relation to the responsibility of each individual for their own (mental) health and well-being."

(Edgley, 2012, p.129)

Despite this close relationship, Edgley (2012) does here draw a point of distinction between recovery and neoliberalism, in contrast to Braslow (2013). Other commentators assert a more clear-cut distinction between recovery and neoliberalism, going so far as to distinguish between neoliberal and non-neoliberal recovery versions (Deegan, 1998; Harper and McKeown, 2018; 
Recovery in the Bin, 2019; Repper and Perkins, 2003; Rose, 2014; Woods et al., 2019). Woods and colleagues (2019) note that the lack of consensus over the definition of recovery is rooted in a fundamental dichotomy between recovery versions that are either top-down policy directives or bottom-up survivor-led programmes of action (see also Recovery in the Bin, 2019). According to Woods and associates (2019), recovery of the latter type originated as a radical idea of the user / survivor movements of the 1970s and 1980s, at the basis of protest against the abuses of institutionalisation and psychopharmaceuticals (Braslow, 2013; Edgley, 2012; Moncrieff, 2006; 2009). More recently, this survivor-led critique has added the notion that the radical idea of recovery has been co-opted by mainstream policy-makers in order to promote a neoliberalist agenda culminating in top-down policy initiatives (Harper and McKeown, 2018; Recovery in the Bin, 2019; Rose, 2014; Woods et al., 2019). According to this critique, neoliberalism is not an intrinsic value system within recovery, since an alternative, bottom-up ideological source for recovery is rooted in user / survivor movements and their emancipatory program.

The third recovery type listed in section 7.2 is representative of this survivor-led critique, being characterised by an emancipatory programme of self-management, particularly in analysis of Dylan's talk in Chapter five and Ian's talk in this chapter. This is also related to the concept, 'Empowerment and self-management' formulated in conceptual analysis (see concept map under the category 'Recovery approaches and constructions' in Figure 7). In the analysis I produced in section 5.2.2 of Dylan's talk, I contrasted this major recovery version, which I understood as an emancipatory critique of services rooted in service users' lived experience, against a more colonised, neoliberal self-management version of recovery promoted by the Mental Health (Wales) Measure 2010. This contrast, reflecting the bottom-up and top-down dichotomy identified by Woods and associates (2019), can be seen to cohere with the dichotomy provided by Habermas of system versus lifeworld. On the one hand, there can be seen to be colonised, neoliberalist policy-versions of recovery, such as the Mental Health (Wales) Measure 2010. On the other hand, there are recovery versions rooted in the lived experience or lifeworld of services users, and a related emancipatory project seeking to prioritise this over psychiatric-medical and neoliberalist dominance. 
Early literature on recovery supports this original idea of recovery grounded in the lived experience of the service user. Patricia Deegan (1988) is keen to emphasise this foundation in her own account of her experience of psychiatric illness:

"Recovery refers to the lived or real life experience of persons as they accept and overcome the challenge of a disability."

(Deegan, 1988, p.55)

Other commentators emphasise the gulf between lived experience and the medicalised approaches of psychiatric professionals (Middleton, 2007; Pilgrim and McCranie, 2013; Repper and Perkins, 2003). Repper and Perkins (2003) argue that the underlying value of recovery should be that the starting point for tackling mental illness is "the voice of first-hand experience" (p.14) not a focus on symptoms and deficits. In section 7.2 above, Tamsen talked about this same focus in her nascent primary care recovery version concerning "living as full a life as you can despite having whatever condition you have" (lines 383 to 384). Anna, a third sector day centre senior support worker I interviewed in the second phase of data collection, describes this view of recovery in her talk about a focus on the person rather than on illness or deficits. This ethos underlies her third sector organisation and its view of attendees with mental health issues:

29. Anna: They don't have to be

30. about their mental illness. It's just about them as people.

31. So I think when they're coming from - whichever - the

32. primary or the secondary - when they're with that

33. professional body - it's all about the illness, it's all

34. about the depression, anxiety, the schizophrenia, the

35. medication - it's about that.

36. But when they're with us it's

37. about being themselves

38. and that's hugely beneficial to them.

[Anna, Senior third sector support worker, Phase 2, Participant 2, lines 29-38]

Anna describes the emphasis of her organisation is on helping service users to "be themselves" (line 37), which is "hugely beneficial to them" (line 38). This is one way of describing the attainment of self-actualisation which Middleton (2007) alludes to in the following statement: 
"The term [recovery] refers to the recovery of a state of self-actualisation, autonomy and relative existential stability, grounded in and reflecting lived experience, rather than a symptoms rating scale."

(Middleton, 2007, p.41)

Here, Middleton (2007), in her discussion paper on critical psychiatry and its relation to the recovery paradigm, succinctly encapsulates the radical, emancipatory idea of recovery expressed by Anna, focused not on directly resolving people's mental health illness but on "being themselves" (line 37). Recovery, according to Middleton (2007), can be understood as moving into this state of self-actualisation or existential authenticity rooted in the lived experience of the service user, in contrast to superimposed policy-versions of recovery, such as neoliberal versions, or the biopsychiatric colonisations, to which Anna refers. Morrow (2013) comments that these top-down versions are closely related since "biomedicalism and neoliberalism are co-constitutive and enhance and support each other ideologically" (p.332). ${ }^{28}$

The dichotomy in this section identified between recovery versions in the form of top-down versions on the one hand and emancipatory versions of recovery rooted in the lived experience of services users therefore has clear parallels with the Habermasian dichotomy of system versus lifeworld, provided above. The key implication I want to make here is that an application of Habermasian theory provides key insight into this dichotomy at the heart of the recovery paradigm, identified by Woods and associates (2019). Originally, it could be said, recovery inhabited the sphere of the lifeworld as a radical emancipatory idea generated from the bottomup by service users and their protest movements. The concept has however to an extent been colonised by an alternative form of liberal ideology in the form of neoliberalism, generating a variety top-down policy programmes. ${ }^{29}$ These initiatives have increasingly disrupted and

\footnotetext{
${ }^{28}$ Biopsychiatric colonisations of recovery (Barker, 2003; Mind, 2008; Morrow, 2013; Szasz, 1974a; 1974b) are also an important form of hegemonization, but are not the main focus in this thesis which focuses on neoliberal, policy-based colonisations.

${ }^{29}$ A potential contradiction may be noted between recovery colonisation which is systemic, identified in this section, and self-oriented recovery versions, identified in section 7.6, which are also colonised but on a much smaller scale, at the ground-level of the practice of service users and workers (Lipsky, 1980). One might object that you can't have colonisation or related processes of hegemony operating in both directions, from the top-down and the bottom-up! However, I would argue that this picture of multi-directional construction and colonisation of recovery versions is tenable and entirely consistent with the view provided by Laclau and Mouffe (1985) of the order of discourse as characterised by ubiquitous hegemony and perpetual discursive struggle. Dominant colonisations, such as neoliberalism, represent dominant ideologies achieving greater hegemony. Against this there are smaller-scale colonisations achieving a lower level of hegemony or fixing of the meaning of the recovery concept, within their micro-social domains. If any of these smaller-scale colonisations became dominant on a
} 
constricted the inherent meaning-making activity of recovery and the normative values this precipitates, as systematic distortion of the communicative sphere has occurred. Deegan (1999) describes this state of affairs as follows:

"Once recovery become systematised, you've got it wrong. Once it is reduced to a set of principles it is wrong. It is a unique and individualised process."

(Deegan, 1999, cited in Repper and Perkins, 2003, p.48)

The attempt to produce a top-down, definitive version of recovery in a neoliberalist form only heightens the underlying principle that recovery cannot be captured in such a way. Since recovery was originally grounded in the lived experience of service users, the hermeneutic realm of the lifeworld, its systemisation fails to capture and ultimately threatens its inherent meaningfulness as a person-centred meaning-making activity. The implications of theory applied here are that this process of constructing recovery versions may be curbed or even expunged by systemic colonisation in the form of policy implementations.

The current state of affairs in Welsh mental health services is relevant to this understanding, because, uniquely amongst devolved administrations in the UK, there has been an implementation of a top-down, colonised, neoliberalist policy-based recovery version in the form of legislation. The implications of the above application of Habermasian theory is that this kind of service engineering will potentially have counterproductive effects for delivering authentically recovery-oriented services, since it is distortive of the underlying meaning of recovery rooted in the lifeworld. As I shall argue in the next section, this also has potential to generate increased service systems complexity. ${ }^{30}$

more macro-scale, they would become systemic since, according to Habermas, in so becoming they are uncoupled from the lifeworld (Chouliaraki and Fairclough, 1999; Pusey, 2002).

${ }^{30}$ It might be objected at this point that the idea that recovery has an authentic meaning rooted in the lifeworld of service users contradicts with the poststructuralist view of recovery colonisation and hegemonization I have been advocating in this chapter. This view centres on the idea that the meaning of recovery is not fixed, which leads to the potential for it to have an unlimited variety of meanings. Partly, this stems from a tension which exists between the different theoretical positions of poststructuralism, on the one hand, where meaning is unfixed and there are no limits to its variability, and the critical theory of Habermas, on the other hand, where meaning is grounded in and constrained by "intersubjective dialogue that establishes identity" (Holub, 1991, p.119). However, I do not think that this tension between theoretical perspectives on meaning constitutes a contradiction. Locating an 


\subsection{Systemic recovery as a key factor for complexity}

In the previous section, I have employed Habermasian theory of system versus lifeworld to provide a theoretical basis for neoliberalist colonisation of the recovery concept. This is informative because it provides a clear theoretical basis to distinguish between two primary types of recovery version. The first of these is rooted in the inherent meanings and normative values grounded in the lifeworld of service users, and an emancipatory project of liberation from structures of power within psychiatry, medicine and neoliberalist forces (Morrow, 2013; Pilgrim and McCranie, 2013). The second of these is a neoliberal colonisation of recovery, which has co-opted the approach by adapting it to neoliberalist agendas of healthcare spending and welfare cutbacks (Mind, 2008; Morrow, 2013; Ramanuj et al., 2015). Whilst I have already argued that the first type may contribute towards escalating complexity, the second type, based on systemic colonisation, may also escalate complexity. The idea in Habermasian theory of the systemic sphere uncoupled from the lifeworld (Chouliaraki and Fairclough, 1999) and governed by strategic rationality is associated with implications of systemic complexity. Inherent within the concept of the system is the idea that it is far too complex for human understanding, which is why it has become uncoupled from the lifeworld. The system refers to those areas of life that have become so complex and specialised that they have become divorced from the hermeneutic site of symbolic interaction within the lifeworld. These elements then reenter the lifeworld as colonising forces (Crossley, 2003). The central idea is that late modernity has seen an overloading of the lifeworld by system elements that have a distorting and complexity-generating effect on social practice or discourse (Layder, 2005). This is one way of describing the potential for systematic distortion of recovery to generate complexity. This occurs for instance with the formation of colonised, policy-based, neoliberalist recovery

\footnotetext{
anchor-point for the meaning of recovery in the lifeworld of service users does not conflict with the poststructuralist principle that meaning is unfixable, because, unlike in the case of systemic colonisations, the recognition of such an anchor is not prescriptive about what the meaning of recovery should be. Rather, this understanding provided by Habermas provides a point of navigation for the meaning-making process of constructing recovery versions to occur which is rooted in the consensus and intersubjectivity of the lifeworld. In this way, combining poststructuralist and Habermasian perspectives on discourse about recovery means that the meaning-making process sees différance as a necessary complement to the search for consensus in the communicative sphere of the lifeworld (Chouliaraki and Fairclough, 1999). The principles of differrance and lifeworld as grounds of meaning can therefore work with one another rather than being contradictory.
} 
versions such as the Mental Health (Wales) Measure, whose escalating impact upon complexity has been shown in this and previous chapters, and particularly in Figures 14 and 15.

An additional understanding of how complexity is introduced into the service system is advanced by making a theoretical link between the concepts of colonisation and hegemony. It should be clear from the above discussion that there are distinct parallels between the idea of colonisation of the lifeworld provided by Habermas (1984b) and the concept of hegemony within Laclau and Mouffe's (1985) discourse theory. Indeed, I regard the theory of colonisation of the recovery concept and hegemonization of the floating signifier to be different theoretical perspectives on the same discursive phenomena surrounding recovery. Colonisation describes how recovery may be invaded and co-opted by external agendas and ideologies, such neoliberalism. Hegemony, in Laclau and Mouffe's (1985) theory, describes how the meaning of recovery is fixed by different discourses as part of the ongoing discursive struggle within the linguistic system. The two concepts therefore describe, from different theoretical perspectives, how recovery may be invaded and co-opted by external agendas and ideologies, though with Laclau and Mouffe's (1985) understanding of hegemony, this co-option is applied within a discursive framework. The connecting of these two theoretical perspectives allows this discursive framework to be applied to colonisation. Since points of hegemony of floating signifiers are points of concentrated complexity generation (see section 7.5), this will also be true of points of systemic colonisation. This may provide one explanation for why imposition of the systemic sphere upon the lifeworld is inherently complicating, because colonisation is also hegemonic. Specifically, this complicating effect is the result of the functionally interpreted systemic realm being imposed upon the hermeneutically understood domain of the lifeworld. Systemic colonisation is therefore an inherently complicating and fragmentary factor for the domain of the lifeworld.

Applying this theory to this study, top-down versions of recovery, in attempting to impose or hegemonize a dominant and final meaning of recovery onto the hermeneutic sphere of the lifeworld do not achieve some sort of final fixing of the meaning of recovery, since this contradicts the continued capacity of the hermeneutic sphere to generate new meaning. Instead, this imposition has a counter-productive effect of generating fragmentation and systemic complexity, phenomena which have been seen in a variety of ways in preceding analysis. I 
have argued in section 7.6 that a proliferation of self-oriented, bottom-up recovery versions may lead to escalating service complexity and fragmentation, and here that the domination of a top-down recovery version may also lead to escalating complexity. Therefore, I conclude that the pre-eminence of either top-down or bottom-up recovery-types, which would represent a polarisation of recovery-based services towards either side of this dichotomy, has the potential to generate escalating complexity and fragmentation in recovery-oriented services.

\subsection{Conclusion}

In this chapter I have argued that my application of theory linking discourse and complexity and the Habermasian notion of colonisation of the lifeworld provides a cogent and cohesive explanation for study findings. My key claims are that recovery should be understood as a person-centred meaning-making activity and that the proliferation of recovery versions this generates is closely allied with escalating service system complexity and fragmentation. Misaligned expectations of transforming services have a similar effect. In addition to this, I have highlighted the detrimental effect that neoliberalist, policy-based colonisation of recovery may have on the capacity for authentic recovery versions to be generated, which are rooted in the lifeworld of service users. This colonisation of the lifeworld may also have a further impact escalating service system complexity and fragmentation.

Building on the poststructuralist underpinning for discourse theory I introduced in sections 3.6.4 and 3.7.1.2, I provided an explanation for why misaligned expectations of services are a complex systems effect, since these will be points of concentration of meaning-making activity as individuals seek to make sense of transforming services. I also took up Laclau and Mouffe's (1985) notion of the floating signifier, applied to the polyvalent recovery concept in section 5.4.1. Since floating signifiers are subject to hegemonic attempts to fix their meaning, colonised recovery versions were seen as points of concentration of meaning-making activity, and therefore of complexity generation. Indeed, recovery versions can be seen as specific ways in which individuals or groups seek to make sense of and fix the meaning of transforming services, and their recovery trajectories within them. More fundamentally, since recovery is a floating signifier, there will be a tendency for recovery versions to be self-oriented, as individuals appropriate the meaning of the concept according to their own "deeply personal" 
(Anthony, 1993, p.527) interpretations. Since hermeneutic and self-oriented versions of recovery will exhibit great diversity, this will act as a catalyst for escalating service system complexity and fragmentation.

This led to my presentation of an emerging, postpsychiatric picture of recovery-oriented Welsh mental health services which are complex, disconnected and fragmented. I saw diverse, selforiented recovery versions as factors for escalating complexity which I viewed as an instance of the difficulty of how emancipated individuals can co-operate with one another without this leading to conflict and disorder.

Finally, I introduced the Habermasian social theory of system versus lifeworld (1984a; 1984b). This was used to broaden the notion of medicalised, psychiatric colonisation to an idea of neoliberalist colonisation of the recovery concept in the form of policy programmes such as the Mental Health (Wales) Measure 2010. I argued that this form of neoliberalist colonisation acts as another key catalyst for service complexity, as well as being distortive of the radical idea of recovery originally rooted in the lifeworld of service users and survivors. I argued that a dichotomy exists as an underlying structure for competing versions of recovery, between a systemic, neoliberalist recovery type and an emancipatory recovery type rooted in the lifeworld of the service user.

On either side of this dichotomy, both top-down, systemic recovery versions and bottom-up self-oriented recovery versions were seen as catalysts for service complexity. Therefore, a preeminence of or polarisation of services towards either top-down or bottom-up recovery versions may stimulate escalating complexity and fragmentation. Complexity and fragmentation engendered in either of these ways has deleterious implications for care continuity, coordination and collaboration. In the concluding chapter which follows, I apply the theory and argument I have built in this chapter as the basis for more concrete proposals which I argue may have the potential to resolve these issues and difficulties facing services in Wales. 


\section{Chapter 8: Study conclusion and implications}

\subsection{Introduction}

In this final chapter, I first outline study limitations, which I follow by describing a theoretical basis for tackling the key issues of service fragmentation and escalating complexity, which have been identified in this thesis, based on the Habermasian notions of 'Public sphere' and 'Ideal speech situation' (Habermas 1970a; 1970b; 1991). This then forms the basis of recommendations for practice, education and policy-implementation and research. These recommendations are intended to assist in preserving the radical idea of recovery from systemic distortion by policy-based neoliberalist colonisations. I advocate that delimiting the colonisation of top-down, policy-based recovery versions in this way leads to the potential for a balance to be struck between top-down and bottom-up recovery versions. Since complexity may be engendered by the pre-eminence of either top-down or bottom-up recovery-types, I argue that offsetting them against one another is a key way to limit complexity generation which would otherwise escalate in recovery-oriented services. Following on from this, I outline the original contribution of this study to understanding recovery-oriented mental health services in Wales. Finally, I provide a concluding statement about recovery-oriented services in view of the key messages of this study.

\subsection{Study limitations}

The most obvious limitation of this research study stems from the various critiques and questions of epistemic value which may be levelled at qualitative research inquiry (Bishop, 2007; Ritchie et al., 2013). Qualitative research is considered to be impressionistic and subjective, with too great a reliance on the researcher's potentially unsystematic views about what is significant and important (Bryman, 2016; Ritchie et al., 2013). This is potentially problematic with regard to analysis and theoretical formation in this thesis. One of the ways I have tried to counter this was to carry out a rigorous approach by providing a systematic context for discourse analysis in the form of a comprehensive conceptual analysis. Another issue is that I may also have been influenced by interpersonal relationships that I struck up with certain participants during the course of interviews. In section 4.3.2.2, I described how I was aware of this tendency towards partiality in relation to Katy's report of perceived difficulties negotiating 
service boundaries. Although, through self-reflection I was able to counter the potential for objectivity to be undermined, I may not have been aware of this same tendency in other cases. Any such omissions of reflexivity may have undermined the epistemological validity of this inquiry. Having said this, I hope that such cases would be a rarity due to the care and attention I paid to reflexivity throughout the course of the project. My attention to reflexivity, rigour and transparency of method means that my interpretative position has predominantly been a valid contributor to the epistemological value of this study, and not a detraction from it.

The location of this study was a relatively small region within Wales, and a broader study over a larger geographical area with a larger sample size might have provided stronger grounds for generalisation. Having said that, I believe that sample size was appropriate for in-depth qualitative analysis (Adler and Adler, 2012; Seale, 2011). The efficacy of purposive maximum variation sampling to ensure heterogeneity of the sample may have been limited by some initial difficulties I had with recruiting willing participants from various third sector venues. In addition to this, it should be said that there is a distinct possibility that the project will have attracted a greater number of people who were critical of services and primary mental healthcare delivery, than those who had more positive views. Interestingly, the predominant mode of discourse for interviews was that of a complaint.

Additionally, the general critique of generalisation for qualitative research may be applied to this study. It is suggested that the scope of findings of qualitative research is restricted (Bryman, 2016). This is potentially problematic since claims and findings I have made can be considered to be quite extensive, as I have sought to provide commentary and analysis upon the entirety of Welsh mental health services. In defence of my approach of generalisation to develop key findings I would say two things. Firstly, I have been at pains to articulate an understanding of the nature of theoretical generalisation I have adopted in this study. Accordingly, I have not sought to generalise directly to make claims about broader settings and populations. Rather, I have made generalisations to theory. I consider that the "cogency of theoretical reasoning" (Mitchell, 1983, p.207) is the basis for the validity of my findings, which have concerned broad theoretical inferences about the distribution of recovery versions throughout discourse, and the impact that this will have on service complexity and care continuity. Secondly, it should be clearly stated that such theoretical generalisation should not be considered to achieve the same 
nature of empirical validity that would occur with quantitative research. Rather, the theorising and theory-building I have undertaken in this thesis to create a cogent explanation for findings should be understood as a form of speculative, though epistemologically valid, proposal about mental health services, and the underpinnings of participant experiences. Qualitative research conceived in this way is truly exploratory (Creswell, 2013; Ritchie et al., 2013), providing reconnaissance at the frontiers of human knowledge, and directions for future research to develop and establish findings with greater precision and certainty.

Finally, it is appropriate to deal with a key criticism of Habermasian theory and its application in this thesis, which might otherwise be considered a key theoretical limitation for this work. The objection from the traditional Marxist camp is that Habermas has reduced politics to communication. As Ian Craib writes, "It sometimes seems that if we could just manage to understand each other better, then everything would be alright. Once again the life and death struggle that makes up much political action is avoided in his theory" (Craib, 1984, p.212). However, I would counter by asserting that issues of communication and discourse are proper grounds for political action, which is inherent in the post-Marxist position I have adopted for this thesis, which grafts poststructuralism onto Marxism in order to free emancipatory politics from an essentialist grounding in economic materialism (Epstein, 1996; Laclau and Mouffe, 1985). In view of this shift inherent in the inauguration of radical democracy, it is in the sphere of communicative action that emancipatory concerns are truly located. This thesis has shown how issues of meaning appropriation are highly relevant to the nature and configuration of recovery-oriented services. Issues of escalating complexity and neoliberal colonisation of the recovery concept have been shown though discourse analysis to operate in and around the realm of human communication. Therefore, the communicative sphere is the appropriate and very real mode of operation for emancipatory struggle related to recovery, the success or failure of which can have life-determining consequences for service users. Defending and nurturing healthy meaning-making contexts for recovery really is an ideological struggle of life or death! In the next section, I introduce Habermasian notions of 'Public sphere' and 'Ideal speech situation' which may be understood to provide underlying principles for nurturing and defending meaning-making contexts within the communicative sphere. 


\subsection{Theoretical solutions: Ideal speech situations and the public sphere}

Habermasian social theory offers two related solutions to the problem of systematically distorted communication: the 'Ideal speech situation' and the 'Public sphere' (Crossley, 2005; Habermas, 1991; Pusey, 2002). In this section I will outline these concepts as an underpinning for recommendations in the next section.

The ideal speech situation was formulated in Habermas' early work (Habermas 1970a; 1970b) and would contribute to the formation of his later theory of the public sphere (Habermas, 1991). The ideal speech situation refers to the "the notion and possibility of a form of discourse composed of the exchange of reasons and steered by the force of the better argument alone" (Crossley, 2005, p.140). This therefore is the free function of communicative action in the sphere of the lifeworld and intersubjective communication between individuals, unencumbered by systemic or colonising forces which might systemically distort communication (Habermas 1970a). This involves curtailing or limiting processes of systemic colonisation. In terms of recovery, this implies that systemically colonised recovery versions should be limited and not allowed to dominate services so that healthier conditions are cultivated for development of recovery versions from the bottom-up. This could be considered to be an idealistic sentiment, but I shall develop this abstract line of thinking into more concrete applications in the next section on study implications and recommendations.

Habermas (1991) later developed this theory about the ideal speech situation into a broader notion of the public sphere. The public sphere denotes a space, real or virtual, in which individuals who might otherwise pursue private, self-interested agendas come together to discuss issues of common concern (Crossley 2005; 2003). The public sphere is therefore a social context of one form or another in which conditions are engineered or encouraged for ideal speech situations to occur. The famous coffee houses and salons which sprung up in key urban centres during the eighteenth centuries are an historical example of the public sphere invoked by Habermas (Crossley, 2003; 2005). Indeed, Habermas sees these venues as prototypes of an effective, modern public sphere (Crossley, 2003). Once again, this concept is expressive of conditions in which the communicative potential of intersubjectivity rooted in 
the lifeworld is emancipated or defended from the hegemony and colonisation of the systemic sphere (Habermas, 2010 [1964]).

However, this defence is not easy to achieve according to Habermas since processes of colonisation are powerful and hegemonic. A vital factor in reclaiming public spheres and ideal speech situations for the lifeworld is the activity of new social movements. New social movements are social movements of protest such as the student movement, as well as environmentalist, feminist, pacifist and animal rights movements (Crossley 2005). In the field of mental health, new social movements may be represented by the user / survivor groups which originally promoted the recovery concept in its radical form. ${ }^{31}$

Having outlined these theoretical areas, I now move onto suggest practical and concrete implementations on the basis of this theory for practice, education, policy and research.

\subsection{Study implications and recommendations}

\subsubsection{Practical recommendations}

The recommendations in this section relate to practical measures which can be undertaken by various groups within local mental health services, across different voluntary and professional sectors, to move toward resolving the issues which have been raised in previous discussion. I will first continue the idea in the previous section that new social movements have a vital role to play in establishing and defending a public sphere for proper consideration and construction of recovery approaches and versions, as well as for preserving recovery concepts.

Habermasian theory provides grounds for seeing user / survivor groups as a major force in reappropriating reified system structures which have encroached upon the recovery concept, such as neoliberal, austerity-based policy forms (Pusey, 2002; Thomas, 2016). This is a crucial point because some factions amongst mental health protest groups have rejected the concept of recovery as either intrinsically suspect (Braslow, 2013; https://recoveryinthebin.org/), or a

\footnotetext{
${ }^{31}$ As was noted in Footnote 24, these new social movements play a key role in promoting the cultural politics of post-Marxism as expounded by Laclau and Mouffe (1985). This represents another parallel between Habermasian theory (1984a; 1984b) and the discourse theory of Laclau and Mouffe (1985).
} 
radical idea that has become so hopelessly colonised that it is now a lost cause (Mind, 2008; Woods et al., 2019). The alternative line for the survivor-led critique of recovery is that this radical idea, rooted in service users' lived experiences, is still intact although it has been considerably assailed by the co-option of policy-makers either implicitly or explicitly pursuing a neoliberalist agenda (Rose, 2014; Woods et al., 2019). According to this view, recovery can and does exist as a legitimate entity so long as it contains an element un-colonised or hegemonized by neoliberalism. The evidence and theoretical analysis of this thesis has contributed the idea that this position is most accurate, since analysis and argument has led to the conclusion that diverse, potentially emancipatory versions of recovery are constructed within the hermeneutic dimension of service users' lived experiences. This points towards the necessity of reclaiming the radical idea from neoliberalist encroachment. In the context of this activity, I would argue that the activism of user / survivor protest groups "harbours the most potential for a real paradigm shift in mental health, that holds a place for recovery as a radical idea" (Morrow, 2013, p.333). This paradigm shift would entail a restoration and reclamation of the original radical idea of recovery rooted in the lifeworld and intersubjectivity of service users.

Social movements within the field of mental health, will have a key role to play in carrying out this task, and consideration of this leads to my initial recommendations. User / survivor groups such as the 'National Survivor User Network' (www.nsun.org.uk), as well as UK-wide and national third sector organisations such as Mind and West Wales Action for Mental Health (WWAMH), have an interest in campaigning on key issues that affect and define people with mental health issues, including recovery (see for instance, WWAMH's 'Recovery Wall' - A digital media venue in which recovery and well-being are promoted http://www.wwamh.org.uk/recoverywall). This thesis provides a theoretical and evidential basis for these groups and their campaigns to focus on defending and promoting legitimate recovery values, rooted in the lived experiences of services users. Campaigning should occur on this grounds, and on the basis of further research which might build on the findings of this thesis (see section 8.4.4), rather than on the basis of a more radical idea of rejecting recovery concepts and approaches outright. 
The second area of practical recommendation I provide concerns the kind of public sphere situations which could be encouraged in the local context, supported by the activism of campaigning groups. These situations would provide positive conditions for the communicative action within the intersubjective level of the lifeworld to generate recovery versions. Peer support groups are one concrete instance of public sphere which may be a fruitful venue for construction of recovery versions. In these groups and venues, life-experiences of difficulties, stigma and recovery may be pooled in the context of reciprocal relationships, in order to promote each other's recovery-journeys (Davidson et al., 2012; Flores, 2001; Flores, 2004; O'Keeffe et al., 2018). Peer support groups supporting mental health recovery may be facilitated within services or the third sector. 'Recovery colleges' are one particular type of support group which could be promoted in local services in order to instantiate recoverypromoting public spheres. Recovery colleges, proposed by Perkins and associates (2012), emphasise recovery-focused peer support and co-production, and may be part of a shift towards recovery-oriented services (Harper and McKeown, 2018; Slade et al., 2014). Initiating and developing Recovery colleges in Wales might therefore be an endeavour which would be worth undertaking in order to promote positive conditions for recovery.

In addition to this, the promotion and nurturing of public spheres would enable an intersubjective pooling of recovery constructions. This would perhaps tackle to some extent the issue identified in section 7.6 of diverse and competing self-oriented recovery generation, which escalates complexity and discontinuity in mental health services. Some degree of harmonisation or cohesion between competing recovery versions could be achieved in the context of ideal speech situations engendered within public sphere venues. This cohesion is greatly preferable to any singular, super-imposed biopsychiatric or policy-based recoveryversion (Morrow, 2013), aimed at achieving greater cohesion of recovery versions. As I have argued, this form of systemic colonisation, encroaches upon and distorts the inherent meanings and normative values of recovery grounded in the lifeworld of service users, (see section 7.9), as well as being anathema to a healthy diversity of recovery versions. As I have also argued in section 7.10, the imposition of the systemic sphere onto that of the lifeworld is also generative of complexity which I have related to service fragmentation. 
Another potential area for encouragement of public sphere venues for recovery is the development of online forums and blogs in which discussion and sharing of lived experiences could occur. These programmes might have the potential to bridge barriers which exist between service sectors which, as this investigation has shown, can lead to barriers to collaboration. For instance, these venues might provide an arena for discussion and sharing of ideas between GPs and third sector workers, in which interpretations and ideas of recovery could be shared and conveyed. This might begin to remedy the situation observed in Chapter five that none of the GP participants could begin to talk about recovery approaches without my providing prior explanation. Interestingly, as soon as I did this, a productive discussion between each GP participant and the researcher ensued, which involved construction of nascent, primary care recovery versions. This kind of activity, which might be seen as an instance of the ideal speech situation, could continue to occur to a much greater extent in the kind of venues I have outlined here.

Additionally, these activities and venues might allow for greater harmony to be nurtured and less oppositionality to rise up between different recovery versions, such as occurred between nascent primary care recovery versions and those of third sector workers like Tony and Hannah (see sections 5.3.1 and 5.4.1). Truth and reconciliation approaches might provide a further, and more immediate conceptual underpinning for this kind of activity, aimed at reducing opposition and barriers between service sectors which have grown up over time due to negative experiences, misunderstanding and a lack of opportunities for communicative engagement (Spandler and McKeown, 2017).

\subsubsection{Implications for education}

The findings of this study may have significant implications for new education and training. One of the most obvious deficiencies in education and training about recovery noted in this study was the lack of knowledge about the concept amongst GP participants. This might lead to the recommendation that training for GPs in mental health or even theories of recovery should be mandatory. That this is not currently the case in the UK is both incongruous and concerning given the commitment to parity of esteem between mental and physical health and care (Health and Social Care Act, 2012; WHO, 2008). The Mental Health (Wales) Measure 
can be seen as specifically implementing this pledge in a devolved administrative context through enhancement of primary care mental health services under Part 1 of the policy. It is difficult to see how this commitment can be properly carried out given that it is not a necessity that GPs have any training or education in mental health and recovery. At the same time, given the power of General Practitioners as a professional body (Baggott, 2007; North and Peckham, 2001), it is difficult to see how policy makers could necessitate this kind of training, although such an action would not be impossible. One remedial strategy might be setting up the kind of public sphere venues which have been described in the previous section. In these contexts, a healthy culture of recovery comprehension might proliferate throughout the community of GPs in a way which is natural and organic, rather than being imposed in a mandatory way by legislators. This approach would be a more diplomatic approach to tackling the limitations of education and training for primary mental health care. It would also be more true to the recommendations of Habermasian social theory where meanings of concepts such as recovery should properly be grounded in an intersubjective community of the lifeworld, rather than being imposed from an authoritative source. Alternatively, if this approach were to prove too weak to produce effective change on its own, a more activist approach might be a suitable, complementary undertaking for user / survivor groups to challenge GPs to engage in such groups and to provide appropriate care for mental ill health along the lines of physical ill health.

Initiating and developing Recovery colleges (Harper and McKeown, 2018; Perkins et al., 2012) in Wales could also have psycho-educative potential for service users. This might create the potential for a greater variety of recovery approaches to be explained to individuals like Patricia and Alun, and broaden recovery interpretations. Slade and colleagues (2014) describe 'Recovery colleges' as recovery education programmes for recovery and reintegration of people with psychiatric disabilities. Pioneered at Boston University in 1984, these have been introduced in the UK, with robust supporting evidence for their efficacy (Boyle and Harris, 2009; Foster et al., 2007). Such colleges could be introduced in Wales with the right level of commitment and funding, with a beneficial effect upon recovery-oriented services. 


\subsubsection{Implications for policy}

All of the above initiatives for education and practice would require greater levels of funding and resources which could be sourced at a policy-level. This could prove problematic given current fiscal constraints. However, in view of the theoretical argumentation which I have presented highlighting the adverse effects of top-down neoliberalist policy-implementations of recovery, existing financial resources might be better directed to these initiatives than to the various costs of developing, publishing and promoting new policies. In this sense, it might be said that less is more, with regard to top-down policy initiatives. Policy-makers would benefit from the recognition, supported by this thesis, that producing new, transformative policy programmes seeking to implement recovery-based services is not the best way to nurture and encourage healthy development of mental health services already situated within the recovery paradigm, and might actually distort this culture, leading to escalating complexity and fragmented services. Alternatively, these pre-existing cultural conditions should be carefully nurtured and cultivated in such a way that they grow steadily and organically from the bottomup. This would be more true to the original radical idea of recovery rooted in the lifeworld of the service user, and a better basis for policy-makers to develop service strategy. Recalling the quotation from Deegan in Chapter seven, "Once recovery become systematised, you've got it wrong" (Deegan, 1999, cited in Repper and Perkins, 2003, p.48). On similar lines, Derek Draper, a political lobbyist and member of a roundtable discussion orchestrated by Mind comments:

"Once a concept is taken up officially, it is a good bet that it will be defined and used in a way that won't reflect the understanding and values of people who framed it and need it. Sadly when bureaucratic organisations go for a concept they can spoil it."

(Mind, 2008, p.13)

In view of this line of thinking, Welsh policy makers might be advised to consider a complete change of strategy in seeking to encourage and nurture contemporary recovery-oriented mental health services, rather than systematically planning and re-engineering services from the topdown. Instead of attempting to capture recovery predominantly through policy, which only systemically distorts the concept and generates greater service complexity and fragmentation, they could seek to engineer conditions within which a healthy balance of diverse and cohesive recovery versions will be allowed to flourish. Investment in bottom-up initiatives such as those 
described in the previous two sections would be one way to achieve this. Senker and Green (2016), discussing recovery approaches for substance abuse and alcohol disorders, reflect this sentiment:

"Commissioners and practitioners should seek to support this level of creativity and autonomy rather than being prescriptive about the recovery approach adopted within services."

(Senker and Green, 2016, p.26)

In relation to this, the Mental Health (Wales) Measure 2010 can be considered to be prescriptive about recovery since it pre-defines recovery in terms of an holistic, CTP approach, a narrow or even invalid understanding of recovery as self-management under primary care and reduced dependency on services. My argument in this thesis has been that this sort of policy approach creates more problems than it solves.

However, I argue on the basis of theory presented above, that more limited (and less costly) policy-implementation might have a delimiting role to play in encouraging the right cultural conditions for recovery-oriented services. One of the key conclusions of analysis in Chapter seven was that complexity and associated fragmentation is generated in two contrasting ways. Firstly, it is generated by a multiplicity of diverse and competing, self-oriented interpretations of recovery from the bottom-up. Secondly complexity is inherent in systemic, neoliberal, colonised policy-versions from the top-down. Potentially, these opposing factors for complexity could be arranged in such a way that they offset one another, limiting their capacity to generate adverse complexity and fragmentation. Top-down neoliberalist recovery versions would have a legitimate role to play in providing a delimitation of bottom-up recovery versions through colonisation. This acceptance of a certain degree of systematisation reflects Habermas' recognition that this is irreversible and not inherently negative, and that the viability of modern society depends on it: "If societies had to constantly reach consensus over everything through argumentation, they could not function" (Chouliaraki and Fairclough, 1999, p.85).

However, it would be important to curtail the extent of this neoliberal, policy delivery of recovery so that it does not squeeze out the potential for bottom-up recovery generation. This squeezing out of recovery versions can be seen in the limited definition of work-as-recovery 
provided by Patricia and Alun. It is also inherent in the somewhat minimalistic idea of recovery as merely being discharged to primary care with withdrawal of previous support (Ramanuj et al., 2015). Major care discontinuities related to this were identified in analysis of Sheryl and Bernadette's talk in section 6.4 and the concept map presented in Figure 13.

I propose therefore that a balance should be struck between top-down and bottom-up recovery versions in order to avoid a polarisation of services to either side of this dichotomy. This balance would create conditions for optimal levels of recovery diversity whilst at the same time limiting potentially damaging complexity and fragmentation, and the sort of misaligned expectations due to conflicting powers and interests exhibited by Alex and Katy in Chapter four. In this way, policy-makers could set the right parameters for cultural and communicative conditions within which to encourage the ongoing process of hermeneutic recoveryconstruction and associated expectations. In this vein, Morrow (2013) advocates recovery as a beneficial paradigm by balancing both its transformative potential for the individual and its ability to provide a framework for mental health system transformation. Pilgrim and McCranie (2013) also allude to a balancing of neoliberal and emancipatory agendas. Reflecting on Kim Hopper's $(2007$; 2008) 'working misunderstanding' of recovery, they describe how emancipatory and neoliberal purposes may be combined:

“In other words, Hopper's 'working misunderstanding' of recovery might conveniently suit both purposes. Maybe the withdrawal of State involvement suits libertarians of right and left. Libertarians of the right want to cut costs and those of the left want to respect diversity of personal expression and of 'lifestyles'. The freedom to be who you are and to live a life worth living on your own terms then becomes part of a new moral regime of recovery. But with freedom comes responsibility and the latter is now that of the patient, not their service providers, if paternalism is abandoned in favour of a flexible negotiated order of mutual decision-making."

(Pilgrim and McCranie, 2013, p.72)

Pilgrim and McCranie (2013) describe therefore a way in which a 'negotiated order' may be found which harmonises the tension found between either side of the dichotomy. In this way, two politically proposed means for resolving the difficulty of how emancipated individuals can avoid conflict and disorder can be combined. The first of these, within neoliberalist ideology, is the 'invisible hand' of the market mechanism (Edgley et al., 2012; Smith, 2001 [1776]). The other means, according to the Habermasian notion of the ideal speech situation, "emphasises 
human rationality, and suggests that when conflicts or disagreements develop they can be resolved by reasoned discussion" (Heywood, 2007, pp.193-194). My suggestion here is that, in order to find the best way forward for recovery-oriented services, activities of policy strategizing and service engineering should focus on balancing these twin organising principles, achieving a right relationship between system and lifeworld. This could be considered to be a form of political pluralism (Berlin, 1969; Heywood, 2007), where two sources of impetus for recovery rooted either in the system or the lifeworld are balanced against one another to achieve an optimum state.

This strategy might not prove to be too costly, since the onus is not on policy planning to deliver solutions in rational and extensive legislative form. Rather, policy design is about forming the right conditions for mental health services and patients to construct versions of recovery. In providing this delimiting approach, boundaries are set to inhibit escalating complexity due to unbridled recovery construction. This role of delimiting escalating recovery versions could also be played by top-down, psychiatric colonisations of recovery, since biomedicalism and neoliberalism are co-constitutive and enhance and support each other ideologically (Morrow, 2013)..$^{32}$

A more constrained approach to neoliberalist recovery implementation would have a remedial effect with regard to tendencies towards psychocompulsion (Friedli and Stearn, 2015; Thomas, 2016), since this would delimit and bridle agendas to reduce benefit claimants and the level of dependency on health services which are motivated by purely neoliberalist ideologies. Instead, greater room would be created for more emancipatory recovery versions to flourish. This would bridle the powerful tendency for recovery to be absorbed and hegemonized wholly into patterns of neoliberalist policy (Edgley et al., 2012). Of course, in order to do this, there would need to be some degree of commitment to an agenda which surpasses merely cutting costs and / or passing public services completely over to neoliberalist forces (Wriston, 1992). There would

\footnotetext{
${ }^{32}$ In this vein, a similar strategy could also be employed to balance bottom-up recovery version generation against biopsychiatric colonisations of recovery. Here, the excesses of self-oriented recovery generation, at the peer group level for instance, would be offset by a certain degree of psychiatric colonisation, perhaps based on evidencebased psychotherapeutic or medical interventions. At the same time, a healthy balance would be achieved between these converse forces, so that recovery rooted in the lived experience of the service user does not become dominated and squeezed out by psychiatric colonisation.
} 
also need to be a more substantive commitment to policy-making than mere 'virtue-signalling' (Bartholomew, 2015) in the form of repeated publishing of policies, with a lack of proper consideration of the effects of implementation and concrete on-the-ground results.

\subsubsection{Implications for research}

As I have already outlined in section 8.2, the findings of this qualitative inquiry are not conclusive. More robust, empirical grounding for findings developed here would need to be developed by subsequent quantitative research (Creswell and Plano Clarke, 2011; Seale, 1999). For instance, further quantitative investigation might be undertaken to investigate the relationship between recovery implementations and care continuity. This research has implied that this relationship may often not be a positive one, since a proliferation of competing recovery versions has the potential to engender complexity and fragmentation at a service-wide level. Further research could examine whether specific dimensions of care continuity and specific kinds of recovery version may have a positive or inverse relationship in different service contexts.

One way that further research could be carried out would be to develop a Patient Reported Outcome Measure or PROM (Black, 2013). This quantitative research project could be based on themes and issues for care continuity and recovery based on this qualitative study (FDA, 2009; Johnson et al., 2010; Johnson et al., 2011). Scales developed could be used to compare care continuity and recovery experiences and to investigate a possible relationship between these areas. Potentially the pre-existing scale 'ReQoL (Keetharuth et al., 2018) could be used to appraise recovery versions and experiences, adapting it to the Welsh service context by adding to it a 'bolt-on' PROM based on this study's findings. This project would be aided by the perspectivist paradigm of the new subjective medicine underpinning this study which is appropriate for PROM development. This research could also potentially be broadened to examine the impact of recovery-based policies on services related to phenomena such as care continuity. This might provide further support for the findings and recommendations of this thesis. 
Finally, based on the understanding which has been developed in this thesis, I would not recommend further research to add to the already extensive evidence base attempting to clarify the recovery concept. A key implication of this piece of research is that the recovery concept does not and should not have a precisely fixed meaning. This concords with the advice of Senker and Green (2016):

"Practitioners and services need to value the individual interpretations of recovery rather than being prescriptive around what it 'should' look like. - it shouldn't be a singular concept."

(Senker and Green, 2016, p.16)

Instead, the testimony of this thesis has been that good recovery-oriented services and healthcare are promoted by creating the best possible conditions and parameters for recovery versions and meanings to be generated, which are in some way anchored in the lived experience and lifeworld of people with mental health issues.

\subsection{Original contribution of this study}

Originally, I considered that new research should justifiably undertaken about recovery and care continuity experiences of service users in Wales, in view of service transformation which was occurring in Welsh services, the impact of devolved policy such as the Mental Health (Wales) Measure, and a shift to primary-led care under the auspices of recovery-orientation. This consideration was enhanced by recognition of the gap in knowledge concerning care continuity and recovery experiences of service users at the primary secondary interface. This thesis has successfully provided new knowledge in all of these areas. Specifically, I offer new knowledge related to the nature of recovery-oriented services in Wales, the relationship this has with care continuity and care coordination experiences across service interfaces, and how these manifest in first-hand accounts of the day to day realities of negotiating shifting service structures. I have shown how recovery versions are manifest in discourse in the form of competing versions in contemporary mental health services. Although research is available examining recovery through discourse analysis, conversation analysis or narrative accounts (see for instance Crowe and Luty, 2005; Femdal, 2018; Harper and Speed, 2012; Rudge and Morse, 2001; Woods et al., 2019), this research is unique in identifying the competing nature of recovery discourses as produced by participants in their everyday experiences of mental 
health services. The application of the poststructuralist discourse theory of Laclau and Mouffe (1985) and Cilliers (1998) to relate the competing nature of recovery versions to service complexity and fragmentation also adds to existing knowledge.

My research is also original in the use of an integrative approach of thematic discourse analysis over a large data set to achieve this finding. My approach combining concept maps with discourse analysis as a way of fulfilling the phases of Braun and Clarke's (2006) thematic analysis is an original contribution to a rigorous, qualitative methodological approach. This builds on the work of Singer and Hunter (1999) and Braun and Clarke (2006) in clarifying the approach of thematic discourse analysis.

This study also offers for the first time new evidence on Welsh mental health services, via the use of discourse analysis of participants' talk about healthcare experiences. The picture I generate of Welsh services as postpsychiatric is a novel and valuable application of the postpsychiatric understanding of mental health services to the local context. My analysis shows how this service culture is characterised by complexity, fragmentation and a predominance of care discontinuities, which is detected in the discourse of participants as they seek to negotiate and make sense of Welsh services. My application of the concept of 'floating signifier' to how recovery is understood and positioned by participants represents a further original contribution of this thesis. The link provided in this thesis between a pre-eminence of either top-down or bottom-up recovery versions with service complexity and care discontinuities is also a novel contribution. This leads to my conclusion that attempting to introduce recovery in a haphazard way, or by choosing to introduce it primarily for the purposes of policy imperatives that may not align with service users expectations of services, may not be beneficial and have counterproductive effects on healthcare systems. I have provided new theoretical clarification of precise mechanisms whereby counterproductive complexity and fragmentation may be produced through competing recovery versions constructed in discourse and prescriptively imposed through policy.

In addition to this, I have developed in a novel way the idea of recovery colonisation (Szasz, 1974a; 1974b) by incorporating Habermasian theory of the colonisation of the lifeworld. I 
connected Habermasian colonisation with hegemony in the sense provided by Laclau and Mouffe (1985) in a way which is theoretically novel. This enabled me to provide a detailed explanation, rooted in poststructuralist discourse theory, for why increased recoveryorientation in services can influence escalating complexity. This also enabled me to clarify concerns already raised by other writers and groups (Edgley, 2012; Morrow, 2013; Perkins and Repper, 2018; Recovery in the Bin, 2019; Rose, 2014; Thomas, 2016) about neoliberalist cooption of recovery approaches by policy-makers.

This has formed the basis for me to be able to make recommendations for practice, education and policy in Wales which have a firm theoretical underpinning. One suggestion for overall policy strategy which may be considered to be original is the pluralistic idea (Berlin, 1969; Heywood, 2007) of offsetting top-down and bottom-up recovery versions in order to achieve balance in services rather than a polarisation which leads to escalating complexity. Although this suggestion is implicit in the discussions of some writers (Heywood, 2007; Pilgrim and McCranie, 2013; Senker and Green, 2016), I believe my original contribution is to provide a clarification of this notion, both in terms of the precision of my theoretical argument and its empirical support which I provide in this study.

Finally, a key contribution of this thesis has been to affirm and preserve the concept of recovery itself. This is potentially a controversial conclusion to arrive at given the critique and campaign to repudiate recovery altogether resting in some quarters. In relation to this I have provided guidance for how the recovery concept may be preserved in services in general, in the light of the vulnerability of this delicate concept to distortion and absorption within disparate agendas, particularly neoliberalism. I shall provide further clarification of this insight in my concluding statement (section 8.6).

\subsection{Concluding statement}

This thesis has investigated service users' and practitioners' experiences of recovery and care continuity to reveal a picture of complex and fragmented mental health services characterised by discontinuities of care. Much of this state of affairs stems from the difficulties involved with recovery-oriented services, particularly when top-down recovery programs are implemented. 
Recovery is a delicate and fragile concept whose meaning should not be fixed other than to affirm and defend its rootedness in the lived experiences of service users. It should also be grounded in their prerogative to provide the final interpretation of their mental illness and their recovery from it. If poorly managed, increased recovery-orientation will not lead to an overall improvement in continuities of care, as might be expected. Rather it may have counterproductive effects such as escalating service complexity and fragmentation, greater levels of care discontinuities, and the hegemony of a narrow, colonised, neoliberalist interpretation of recovery. These effects have the potential to leave service users bereft of the treatment and support that they need to recover. The banner of recovery is not therefore a panacea for all the challenges of delivering effective mental health services. Recovery must be implemented with care, giving room for the paradigm to develop organically from within the hermeneutic and intersubjective dimension of service users' lives. Otherwise, if the ill-effects of poor implementation are left unchecked, scepticism will overtake positive attitudes towards recovery, leading to a repudiation of the concept, and society will lose this vital and fundamental approach for tackling mental illness. 


\section{References}

Abercrombie, N. and Turner, B. S. (1978). The dominant ideology thesis. British Journal of Sociology, 149-170.

Adair, C. E., McDougall, G. M., Beckie, A., Joyce, A., Mitton, C., Wild, C. T., Gordon, A. \& Costigan, N. (2003). History and measurement of continuity of care in mental health services and evidence of its role in outcomes. Psychiatric Services, 54, 1351-6.

Adair, C. E., McDougall, G. M., Mitton, C. R., Joyce, A. S., Wild, T. C., Gordon, A., Costigan, N., Kowalsky, L., Pasmeny, G. \& Beckie, A. (2005). Continuity of care and health outcomes among persons with severe mental illness. Psychiatric Services, 56(9), 1061-1069. doi: http://dx.doi.org/10.1176/appi.ps.56.9.1061

Adler, P. A. and Adler, P. (2012). Contribution to S. E. Baker and R. Edwards (eds.), How many qualitative interviews is enough? Expert voices and early career reflections on sampling and cases in qualitative research. National Centre for Research Methods Review Paper, pp.8-11, viewed 08 August 2019, http://eprints.ncrm.ac.uk/2273/

Alcock, P. (2016). 'What is social policy?' in P. Alcock, T. Haux, M. May \& S. Wright (eds.), The student's companion to social policy (5th ed.) (pp.7-13). London: Wiley-Blackwell.

Allen, D. (2009). From boundary concept to boundary object: the practice and politics of care pathway development. Social Science \& Medicine, 69(3), 354-361.

Althusser, L. (1971). 'Ideology and ideological state apparatuses' in L. Althusser (ed.), Lenin and philosophy and other essays. London: New Left Review.

Amering, M. and Schmolke, M. (2009). Recovery in mental health: reshaping scientific and clinical responsibilities. John Wiley \& Sons.

Andrews, T. (2012). What is social constructionism. Grounded Theory Review, 11(1), 39-46.

Antaki, C., and Widdicombe, S. (eds.) (2008). Identities in talk. London: SAGE.

Antaki, C., Billig, M., Edwards, D. \& Potter, J. (2003). Discourse analysis means doing analysis: a critique of six analytic shortcomings. Discourse Analysis Online, 1.

Anthony, W. A. (1993). Recovery from mental illness: the guiding vision of the mental health service system in the 1990s. Psychosocial Rehabilitation Journal, 16(4), 11.

APA: American Psychiatric Association. (2000). Diagnostic and statistical manual of mental disorders: text revision (DSM-4 TRß). Washington DC: American Psychiatric Association Press.

APA: American Psychiatric Association. (2013). Diagnostic and statistical manual of mental disorders (DSM-5®). Washington DC: American Psychiatric Association Press.

Archer, M. S. (1995). Realist social theory: the morphogenetic approach. Cambridge: Cambridge University Press. 
Archer, M. S. (2003). Structure, Agency and the Internal Conversation. Cambridge: Cambridge University Press.

Arksey, H. and O’Malley, L. (2005). Scoping studies: towards a methodological framework. International Journal of Social Research Methodology, 8, 19-32.

Asadi-Lari, M., Tamburini, M. \& Gray, D. (2004). Patients' needs, satisfaction, and health related quality of life: Towards a comprehensive model. Health And Quality Of Life Outcomes, 2, 32.

Atkinson, P., Coffey, A. \& Delamont, S. (2003). Key themes in qualitative research: Continuities and changes. Oxford: Altamira Press.

Austin, J. L. (1975). How to do things with words (2nd ed.). J.O. Urmson and M. Sbisn (Eds) Oxford, Clarendon Press.

Aveyard, H. (2007). Doing a Literature Review. Maidenhead: McGraw-Hill.

Aylward, M., Phillips, C. \& Howson, H. (2013). Simply Prudent Healthcare - achieving better care and value for money in Wales - discussion paper. Cardiff: The Bevan Commission.

Ayres, L., Kavanaugh, K. \& Knafl, K. A. (2003). Within-case and across-case approaches to qualitative data analysis. Qualitative Health Research, 13(6), 871-883.

Bachrach, L. (1981). Continuity of care for chronic mental patients: a conceptual analysis. The American Journal of Psychiatry, 138(11), 1449-1457.

Bachrach L. (1993). 'Continuity of care: a context for case management' in M. Harris and H. Bergman (eds.), Case management for mentally ill patients: theory and practice. Amsterdam: Harwood Academic Publishers/Gordon.

Baggott, R. (2007). Understanding health policy. Bristol: Policy Press.

Baggott, R. (2016). 'Healthcare' in P. Alcock, T. Haux, M. May \& S. Wright (eds.), The student's companion to social policy (5th ed.) (pp.351-357). London: Wiley-Blackwell.

Bakhtin, M. M. (1981). The dialogic imagination: four essays by M.M. Bakhtin, M. Holquist (ed.), trans. C. Emerson and M. Holquist. Austin: University of Texas Press.

Bakhtin, M. M. (2010). Speech genres and other late essays. Austin: University of Texas Press.

Balanchandra, K., Sharma, V., Dozois, D. \& Bhayana, B. (2005). How bipolar disorders are managed in family practice: self-assessment survey. Canadian Family Physician, 51(4), 534-535.

Barker, N. and Lamble, S. (2009). From social security to individual responsibility: Sanctions, conditionality and punitiveness in the Welfare Reform Bill 2009 (Part One) Current developments. Journal of Social Welfare \& Family Law, 31(3), 321-332.

Barker, P. (2003). The Tidal Model: Psychiatric colonization, recovery and the paradigm shift in mental health care. International Journal of Mental Health Nursing, 12(2), 96-102.

Barnett-Page, E. and Thomas, J. (2009). Methods for the synthesis of qualitative research: a critical review. BMC Medical Research Methodology, 9, 59. 
Barrett, M. (1991). 'Ideology, politics, hegemony: from Gramsci to Laclau and Mouffe' in M. Barrett (ed.), The politics of truth: from Marx to Foucault. Cambridge: Polity Press.

Barriball, K. L. (1994). Collecting data using a semi-structured interview a discussion paper. Journal of Advanced Nursing-Institutional Subscription, 19(2), 328-335.

Barry, A.-M. and Yuill, C. (2016). Understanding the sociology of health (4th ed.). Los Angeles: SAGE.

Bartholomew, J. (2015). 'The awful rise of 'virtue signalling', The Spectator, 18 April, https://www.spectator.co.uk/2015/04/hating-the-daily-mail-is-a-substitute-for-doing-good/

Baruch, G. (1981). Moral tales: parents' stories of encounters with the health professions. Sociology of Health \& Illness, 3(3), 275-295.

Bateson, G., Jackson, D. D., Haley, J. \& Weakland, J. (1956). Toward a theory of schizophrenia. Behavioral science, 1(4), 251-264.

Beauchamp, T. and Childress, J. (2013). Principles of Biomedical Ethics. New York: Open University Press.

Beck, U. (1996). World risk society as cosmopolitan society? Ecological questions in a framework of manufactured uncertainties. Theory, Culture \& Society, 13(4), 1-32.

Becker, E., Hayllay, O. \& Wood, M. (2010) Pathways to Work: programme engagement and work patterns. London: Department for Work and Pensions.

Becker, M., Cunningham, C.E., Christensen, B.K., Furimsky, I., Rimas, H., Wilson, F., Jeffs, L., Madsen, V., Bieling, P., Chen, Y. and Mielko, S. (2019). Investigating service features to sustain engagement in early intervention mental health services. Early Intervention in Psychiatry, 13(2), 241-250.

Beck-Sander, A. (1998). Is insight into psychosis meaningful? Journal of Mental Health, 7(1): 25-34.

Belling, R., Whittock, M., McLaren, S., Burns, T., Catty, J., Jones, I. R., Rose, D. and Wykes, T. (2011). Achieving continuity of care: facilitators and barriers in community mental health teams. Implementation Science, 6(1), 23. doi: http://dx.doi.org/10.1186/1748-5908-6-23

Belsey, C. (2002). Poststructuralism: A very short introduction. Oxford: Oxford University Press.

Benwell, B. and Stokoe, E. (2006). Discourse and identity. Edinburgh: Edinburgh University Press.

Berger, P. L. and Luckmann, T. (1991). The social construction of reality: A treatise in the sociology of knowledge. London: Penguin.

Berlin, I. (1969). 'Two concepts of liberty' in I. Berlin, Four essays on liberty (pp.121-54). Oxford: Clarendon Press.

Bernstein, R. J. (1976). The restructuring of social and political theory. Oxford: Blackwell.

Bhaskar, R. (1978). A realist theory of science. Hassocks: Harvester Press.

Bhaskar, R. (1998). The possibility of naturalism a philosophical critique of the contemporary human sciences (3rd ed.). New York: Routledge. 
Billig, M., Condor, S., Edwards, D., Gane, M., Middleton, D. \& Radley, A. (1988). Ideological dilemmas: A social psychology of everyday thinking. London: SAGE.

Bird, C. M. (2005). How I stopped dreading and learned to love transcription. Qualitative Inquiry, 11, 226-248.

Biringer, E., Hartveit, M., Sundfør, B., Ruud, T. \& Borg, M. (2017). Continuity of care as experienced by mental health service users - a qualitative study. BMC Health Services Research, 17(1), 763. doi: http://dx.doi.org/10.1186/s12913-017-2719-9

Bishop, R. (2007). The philosophy of the social sciences : an introduction. London: Continuum.

Black, N. (2013). Patient reported outcome measures could help transform healthcare. British Medical Journal, 346. doi: http://dx.doi.org/10.1136/bmj.f167

Blane, D. (2014). Psychological therapies in primary care: a progress report. British Journal of General Practice, 64, 556-557.

Blumer, H. (1986). Symbolic interactionism: Perspective and method. Berkeley: University of California Press.

BMA: British Medical Association. (2003). New GMS contract 2003 - Investing in general practice. London: British Medical Association.

BMA: British Medical Association. (2018). Focus on the Welsh GP Contract 2017/18. London: British Medical Association.

Bonney, S. and Stickley, T. (2008). Recovery and mental health: a review of the British literature. Journal of Psychiatric and Mental Health Nursing, 15(2), 140-153.

Bowen, G. A. (2008). Naturalistic inquiry and the saturation concept: a research note. Qualitative Research, 8, 137-152.

Bowen, M. (2019). Stigma: a linguistic analysis of personality disorder in the UK popular press, 20082017. Journal of Psychiatric and Mental Health Nursing, 00:1-10.

Bowen, M. and Lovell, A. (2013). Representations of mental health disorders in print media. British Journal of Mental Health Nursing, 2(4), 198-202.

Bowlby, J. (2012). A secure base. London: Routledge.

Bowling, A. (2005). Measuring Health: A Review of Quality of Life Measurement Scales. Maidenhead: McGraw-Hill.

Boyatzis, R. E. (1998). Transforming qualitative information: Thematic analysis and code development. Thousand Oaks, CA: SAGE.

Boyle D. and Harris M. (2009). The challenge of co-production. London: New Economics Foundation.

Bracken, P. and Thomas, P. (2001). Postpsychiatry: a new direction for mental health. British Medical Journal, 322(7288), 724. 
Bracken, P., Thomas, P., Timimi, S., Asen, E., Behr, G., Beuster, C., Bhunnoo, S., Browne, I., Chhina, N., Double, D. \& Downer, S. (2012). Psychiatry beyond the current paradigm. The British Journal of Psychiatry, 201(6), 430-434.

Braslow, J. T. (2013). The manufacture of recovery. Annual review of clinical psychology, 9, 781-809. doi: http://dx.doi.org/10.1146/annurev-clinpsy-050212-185642

Braun, V. and Clarke, V. (2006). Using thematic analysis in psychology. Qualitative Research in Psychology, 3(2), 77-101. doi: http://dx.doi.org/10.1191/1478088706qp063oa

Braun, V., Clarke, V. \& Terry, G. (2014). Thematic analysis. Qualitative Research in Clinical Health Psychology, 24, 95-114.

Brooker, C., Ricketts, T., Bennett, S. \& Lemme, F. (2007). Admission decisions following contact with an emergency mental health assessment and intervention service. Journal of Clinical Nursing, 16(7), 1313-1322.

Brown, P. (1995). Naming and framing: The social construction of diagnosis and illness. Journal of Health and Social Behavior, 34-52.

Bryman, A. (2016). Social research methods (5th ed.). Oxford: Oxford University Press.

BSA: British Sociological Association. (2002). Statement of Ethical Practice for the British Sociological Association. Durham: British Sociological Association.

Bullock, A. and Trombley, S. (eds.) (1999). The new Fontana dictionary of modern thought. ( $3^{\text {rd }}$ ed.). London: HarperCollins.

Burns, T., Catty, J., Clement, S., Harvey, K., Rees Jones, I., McLaren, S., Rose, D., White, S. \& Wykes, T. (2007). Experiences of continuity of care and health and social outcomes: the ECHO study. London: NCCSDO.

Burns, T., Catty, J., Harvey, K., White, S., Jones, I. R., McLaren, S., Wykes, T. \& Group, E. (2013). Continuity of care for carers of people with severe mental illness: results of a longitudinal study. The International Journal of Social Psychiatry, 59, 663-70.

Burns, T., Catty, J., White, S., Clement, S., Ellis, G., Jones, I. R., Lissouba, P., McLaren, S., Rose, D. $\&$ Wykes, T. (2009). Continuity of care in mental health: understanding and measuring a complex phenomenon. Psychological Medicine, 39(2), 313-323. doi:10.1017/S0033291708003747

Burr, V. (2003). Social constructionism (2nd ed.). London: Routledge.

Byrne, D. (1998). Complexity theory and the social sciences: An introduction. London: Routledge.

Campbell, D. (2017). 'NHS bosses warn of mental health crisis with long waits for treatment', The Guardian, 7 July, https://www.theguardian.com/society/2017/jul/07/nhs-bosses-warn-ofmental-health-crisis-with-long-waits-for-treatment

CASP UK (2013). Critical Appraisal Skills Programme: Making Sense of the Evidence, viewed 15 August 2016, https://casp-uk.net/ 
Catty, J., White, S., Clement, S., Cowan, N., Geyer, C., Harvey, K., Jones, I. R., McLaren, S., Poole, Z., Rose, D. \& Wykes, T. (2013). Continuity of care for people with psychotic illness: Its relationship to clinical and social functioning. International Journal of Social Psychiatry, 59(1), 5-17. doi: http://dx.doi.org/10.1177/0020764011421440

Chapman, J. (2004). Systems failure: Why governments must learn to think differently (2nd ed.). London: Demos.

Charlton, R. (2005). Implications of the new GP contract. Clinical Medicine, 5(1), 50-54.

Chen, M. and Lawrie, S. (2017). Newspaper depictions of mental and physical health. BJPsych Bulletin, 41(6), 308-313. doi: http://dx.doi.org/10.1192/pb.bp.116.054775

Chouliaraki, L. and Fairclough, N. (1999). Discourse in late modernity: Rethinking critical discourse analysis: Edinburgh University Press.

Cilliers, P. (1998). Complexity and postmodernism. London: Routledge.

Clarke, L. (2003). Challenging ideas in psychiatric nursing. London: Routledge.

Coffey, A. \& Atkinson, P. (1996). Making sense of qualitative data : complimentary research strategies. London: Thousand Oaks.

Coffey, M., Cohen, R., Faulkner, A., Hannigan, B., Simpson, A. \& Barlow, S. (2017a). Ordinary risks and accepted fictions: how contrasting and competing priorities work in risk assessment and mental health care planning. Health Expectations 20(3), 471-483. doi: http://dx.doi.org/10.1111/hex.12474

Coffey, M., Hannigan, B. \& Simpson, A. (2017b). Care planning and coordination: Imperfect solutions in a complex world. Journal of Psychiatric and Mental Health Nursing, 24(6), 333-334.

Coid, J. (1994). Failure in community care: psychiatry's dilemma. British Medical Journal, 308(6932), 805.

Connell, J., Brazier, J., O’Cathain, A., Lloyd-Jones, M. \& Paisley, S. (2012). Quality of life of people with mental health problems: a synthesis of qualitative research. Health and Quality of Life Outcomes, 10(1), 138.

Connell, J., O'Cathain, A. \& Brazier, J. (2014). Measuring quality of life in mental health: Are we asking the right questions? Social Science \& Medicine, 120, 12-20.

Cooper, D. (1999). Existentialism : a reconstruction. Oxford: Blackwell.

Copeland, M. E. (2000). WRAP: Wellness recovery action plan. Peach Press.

Corbin, J. M. and Strauss, A. (2008). Basics of qualitative research: Techniques and procedures for developing grounded theory. London: SAGE.

Court, C. (1994). Clunis inquiry cites “catalogue of failure". British Medical Journal, 308, 613.

Craib, I. (1984). Modern social theory: from Parsons to Habermas ( $1^{\text {st }}$ ed.). Hemel Hempstead: Wheatsheaf Books. 
Craib, I. (1992). Modern social theory: from Parsons to Habermas (2 ${ }^{\text {nd }}$ ed.). Edinburgh: Pearson Education.

Crawford, M. J., de Jonge, E., Freeman, G. K. \& Weaver, T. (2004). Providing continuity of care for people with severe mental illness- a narrative review. Social Psychiatry and Psychiatric Epidemiology, 39(4), 265-272. doi: http://dx.doi.org/10.1007/s00127-004-0732-x

Crawford, M., Robotham, D., Thana, L., Patterson, S., Weaver, T., Barber, R. \& Wykes, T. (2011). Selecting outcome measures in mental health: The views of service users. Journal of Mental Health, 20(4), 336-346.

Creswell, J. (2013). Research Design: Qualitative, Quantitative and Mixed Methods Approaches. London: SAGE.

Creswell, J. W. and Plano Clark, V. L. (2011). Designing and conducting mixed methods research. (2nd ed.). London: SAGE.

Croce, B. (1914). Historical Materialism and the Economics of Karl Marx, trans. C.M. Meredith, with an Introduction by A.D. Lindsay. London: Allen \& Unwin.

Crossley, N. (2003). Even newer social movements? Anti-corporate protests, capitalist crises and the remoralization of society. The Interdisciplinary Journal of Organization, 10(2), 287-305.

Crossley, N. (2005). Key concepts in critical social theory. London: London : SAGE.

Crotty, M. (1998). The foundations of social research : meaning and perspective in the research process. London: SAGE.

Crowe, M. and Luty, S. (2005). Recovery from depression: a discourse analysis of interpersonal psychotherapy. Nursing Inquiry, 12(1), 43-50.

Currid, T. J., Turner, A., Bellefontaine, N. \& Spada, M. M. (2012). Mental health issues in primary care: implementing policies in practice. British Journal of Community Nursing, 17(1), 21-26.

Dale, R. (1989). The state and education policy. Milton Keynes: Open University.

Danermark, B., Ekstron, M., Jakobsen, L. \& Karlsson, J. (2001). Explaining society: Critical realism in the social sciences. London: Routledge.

Davidson, L. (2005). Recovery, self management and the expert patient - Changing the culture of mental health from a UK perspective. Journal of Mental Health, 14(1), 25-35.

Davidson, L., Bellamy, C., Guy, K. \& Miller, R. (2012). Peer support among persons with severe mental illnesses: a review of evidence and experience. World Psychiatry, 11(2), 123-128.

Davidson, L., O'connell, M. J., Tondora, J., Lawless, M. \& Evans, A. C. (2005). Recovery in serious mental illness: A new wine or just a new bottle? Professional Psychology: Research and Practice, 36(5), 480.

Davies, B. and Harré, R. (1990). Positioning: The discursive production of selves. Journal for the Theory of Social Behaviour, 20(1), 43-63. 
Davis, K., Drey, N., and Gould, D. (2009). What are scoping studies? A review of the nursing literature. International Journal of Nursing Studies, 46, 1386-1400.

De Fina, A. D. (2012). 'Discourse and identity' in T. A. Van Dijk (ed.), Discourse studies: a multidisciplinary introduction (pp.263-282). London: SAGE.

Deegan, P. E. (1988). Recovery: The lived experience of rehabilitation. Psychosocial Rehabilitation Journal, 11(4), 11.

Deegan, P. E. (1993). Recovering our sense of value after being labeled: Mentally ill. Journal of Psychosocial Nursing and Mental Health Services, 31(4), 7-9.

Deegan, P.E. (1999). Recovery: an alien concept. In Proceedings of the Strangefish Conference. Chamberlin Hotel, Birmingham UK.

Delanty, G. (2005). Social science: philosophical and methodological foundations (2nd ed.). Maidenhead: Open University Press.

Delanty, G. and Strydom, P. (eds.) (2003). Philosophies of social science: The classic and contemporary readings. Maidenhead: McGraw-Hill.

Denzin, N. K. (2016). The qualitative manifesto: A call to arms. London: Routledge.

Denzin, N. K. and Giardina, M. D. (2008). Qualitative inquiry and the politics of evidence. Left Coast Press.

Denzin, N. K. and Lincoln, Y. S. (1994). 'Introduction: Entering the field of qualitative research' in N. K. Denzin and Y. S. Lincoln (eds.), Handbook of qualitative research (4th ed.). London: SAGE.

Denzin, N. K. and Lincoln, Y. S. (2017). 'Introduction: The discipline and practice of qualitative research' in N. K. Denzin and Y. S. Lincoln (eds.), The Sage handbook of qualitative research (5th ed.) (pp.1-26). Thousand Oaks, CA: SAGE.

Department of Health and Human Sciences. (2003). Achieving the promise: Transforming mental healthcare in America, President's new freedom commission on mental health. Rockville, MD: Department of Health and Human Sciences.

Department of Health Social Services and Public Safety (2007). Mental health service framework: Project initiation document. Belfast: Department of Health, Social Services and Public Safety.

Derrida, J. (1976). Of Grammatology. Baltimore: Johns Hopkins University Press.

Derrida, J. (1978). Writing and Difference. Chicago: University of Chicago Press.

Dey, I. (1999). Grounding grounded theory: Guidelines for Qualitative Inquiry. London: Academic Press.

Dickerson, F. B. (2006). Commentary: disquieting aspects of the recovery paradigm. Psychiatric Services, 57(5), 647-647.

Dilthey, W. (1976). Selected writings of Wilhelm Dilthey, trans. H.P. Rickman (ed.), with an Introduction by H.P. Rickman. Cambridge : Cambridge University Press. 
Dingwall, R. (1997). 'Accounts, interviews and observations' in M. Gale and R. Dingwall (eds.), Context and method in qualitative research. London: SAGE.

Docherty, J. D. (1997). Barriers to the diagnosis of depression in primary care. Journal of Clinical Psychology, 58, 5-10.

DoH: Department of Health. (1999). National Service Framework for Mental Health: Modern Standards and Service Models. London: Department of Health.

DoH: Department of Health. (2001a). The expert patient: a new approach to chronic disease management for the 21st century. London: Department of Health.

DoH: Department of Health. (2001b). The mental health policy implementation guide. London: Department of Health.

DoH: Department of Health. (2009) New Horizons. London: Department of Health.

Double, D. B. (2006a). 'Critical psychiatry: Challenging the biomedical dominance of psychiatry' in D. B. Double (ed.), Critical psychiatry: the limits of madness (pp.3-15). New York: Palgrave Macmillan.

Double, D. B. (2006b). 'Critical psychiatry and conflict: renewing mental health practice' in D. B. Double (ed.), Critical psychiatry: the limits of madness (pp.209-226). New York: Palgrave Macmillan.

Double, D. B. (2009). Critical psychiatry seeks to avoid the polarisation engendered by anti-psychiatry. Psychiatric Bulletin, 33(10), 395-397.

Drew, P. and Holt, E. (1988). Complainable matters: The use of idiomatic expressions in making complaints. Social Problems, 35(4), 398-417.

Durbin, A., Durbin, J., Hensel, J. M. \& Deber, R. (2016). Barriers and enablers to integrating mental health into primary care: a policy analysis. The Journal of Behavioral Health Services \& Research, 43(1), 127-139.

Durbin, J., Goering, P., Streiner, D. L. \& Pink, G. (2004). Continuity of care: Validation of a new selfreport measure for individuals using mental health services. Journal of Behavioral Health Services \& Research, 31(3), 279-296.

Durkheim, É. (1982 [1938]). The rules of sociological method and selected texts on sociology and its method, S. Lukes (ed.), trans. W.D. Halls, with an Introduction by Steven Lukes. New York: The Free Press.

Edgley, A., Stickley, T., Wright, N. \& Repper, J. (2012). The politics of recovery in mental health: A left libertarian policy analysis. Social Theory \& Health, 10(2), 121-140.

Edley, N. (2001). 'Analysing masculinity: interpretative repertoires, ideological dilemmas and subject positions' in M. Wetherell, S. Taylor and S. Yates (eds.), Discourse as data: a guide for analysis (pp.189-228). London: SAGE, in association with the Open University. 
Edwards, D. (1994). Script formulations: An analysis of event descriptions in conversation. Journal of Language and Social Psychology, 13(3), 211-247.

Edwards, D. (2005). Moaning, whinging and laughing: The subjective side of complaints. Discourse Studies, 7(1), 5-29.

Edwards, D. and Potter, J. (1992). Discursive psychology. London: SAGE.

Edwards, D. and Potter, J. (2001). 'Discursive psychology' in A. W. McHoul and M. Rapley (eds.), How to analyse talk in institutional settings: a casebook of methods. London: Continuum.

England, E., Nash, V. \& Hawthorne, K. (2017). GP training in mental health needs urgent reform. British Medical Journal, 356:j1311. doi: http://dx.doi.org/10.1136/bmj.j1311

Epstein, B. (1996). 'Radical Democracy and Cultural Politics: What about Class? What about Political Power?' in D. Trend (ed.), Radical democracy: identity, citizenship, and the state. London: Routledge.

Epstein, M. (2011). 'Introduction to the Philosophy of Science' in C. Seale (ed.), Researching society and culture (pp.7-28). London: SAGE.

Erteschik-Shir, N. (2007). Information structure: the syntax-discourse interface. Oxford: Oxford University Press.

ESRC: Economic \& Social Research Council. (2015). ESRC Framework for Research Ethics Updated January 2015. Swindon: ESRC.

Etherington, K. (2004). Becoming a reflexive researcher using our selves in research. London: Jessica Kingsley Publishers.

Evans, D. (2002). Database searches for qualitative research. Journal of the Medical Library Association, 90, 77-84.

Fairclough, N. (1992a). Discourse and social change. Cambridge: Polity Press.

Fairclough, N. (1992b). Discourse and text: Linguistic and intertextual analysis within discourse analysis. Discourse \& Society, 3(2), 193-217.

Fairclough, N. (1995). Media Discourse. London: Edward Arnold.

Fairclough, N. (1998). Political discourse in the media: an analytical framework' in A. Bell and P. Garrett (eds.), Approaches to media discourse. Oxford: Blackwell.

Fairclough, N. (2003). Analysing discourse: Textual analysis for social research. London: Routledge.

Fairclough, N. (2013). Critical discourse analysis: The critical study of language. London: Routledge.

FDA: Food and Drug Administration (2009). Guidance for industry: patient-reported outcome measures: use in medical product development to support labeling claims. Federal Register, 74(235), 65132-65133. 
Femdal, I. (2018). The right place? users and professionals' constructions of the place's influence on personal recovery in community mental health services. International Journal of Mental Health Systems, 12(1), 26. doi: http://dx.doi.org/10.1186/s13033-018-0209-z

Fine, M. (1992). Disruptive voices: The possibilities of feminist research. Michigan: University of Michigan Press.

Finlay, L. (2002). "Outing" the researcher: the provenance, process, and practice of reflexivity. Qualitative Health Research, 12(4), 531.

Fleury, M.-J. and Grenier, G. (2011). 'Primary mental healthcare and integrated services' in L. L'Abate (ed.), Mental illnesses - evaluation, treatments and implications. Rijeka, Croatie: InTech.

Flores, P. J. (2001). Addiction as an attachment disorder: Implications for group therapy. International Journal of Group Psychotherapy, 51(1: Special issue), 63-81.

Flores, P. J. (2004). Addiction as an attachment disorder. Maryland: Jason Aronson.

Forrest, E. (2004). Clinical management special: mental health. Right on time. The Health Service Journal, 114(5899), 26-27.

Forrester J. (1968). Principles of Systems. Cambridge: Pegasus Communications.

Foster, G., Taylor, S. J., Eldridge, S., Ramsay, J. \& Griffiths, C. J. (2007). Self-management education programmes by lay leaders for people with chronic conditions. Cochrane Database of Systematic Reviews, (4).

Foucault, M. (1972). The Archaeology of Knowledge.London: Routledge.

Foucault, M. (1973). The Birth of the Clinic: An Archaeology of Medical Perception: New York, NY: Vintage Books.

Freeman, G., Shepperd, S., Robinson, I., Ehrich, K., Richards, S. \& Pittman, P. (2000). Continuity of care: Report of a scoping exercise for the SDO programme of NHS R\&D. London: National Coordinating Centre for Service Delivery and Organisation.

Freeman, G., Weaver, T., Low, J. \& de Jonge, E. (2002). Promoting continuity of care for people with severe mental illness. London: National Co-ordinating Centre for Service Delivery and Organisation.

Friedli, L. and Parsonage, M. (2009). Promoting mental health and preventing mental illness: the economic case for investment in Wales. Cardiff: All Wales Mental Health Promotion Network.

Friedli, L. and Stearn, R. (2015). Positive affect as coercive strategy: conditionality, activation and the role of psychology in UK government workfare programmes. Medical Humanities, 41(1), 40-47.

Fuat Firat, A. and Shultz, C. J. (1997). From segmentation to fragmentation: markets and marketing strategy in the postmodern era. European Journal of Marketing, 31(3/4), 183-207.

Gadamer, H. G. (1989 [1975]). Truth and Method (2nd ed.), trans. W. Glen-Doepel, trans. Revised J. Weinsheimer and D. G. Marshall. London: Sheed and Ward. 
Gamble, A. (1994). The free economy and the strong state: The politics of Thatcherism (2nd ed.). London: Macmillan Press.

Gask, L., Rogers, A., Oliver, D., May, C. \& Roland, M. (2003). Qualitative study of patients' perceptions of the quality of care for depression in general practice. British Journal of General Practice, 53(489), 278-283.

Gask, L., Sibbald, B. \& Creed, F. (1997). Evaluating models of working at the interface between mental health services and primary care. British Journal of Psychiatry, 170(1), 6-11. doi: http://dx.doi.org/10.1192/bjp.170.1.6

Gee, J. P. (2004). An introduction to discourse analysis: Theory and method. London: Routledge.

Gee, J. P. (2008). Social linguistics and literacies: Ideology in discourses. London: Routledge.

Gee, J. P. (2010). How to do discourse analysis: A toolkit. London: Routledge.

Gergen, K. J. (1994). Toward transformation in social knowledge (2nd ed.). Thousand Oaks, CA: SAGE.

Giddens, A. (1984). 'Social science as a double hermeneutic' in G. Delanty and P. Strydom (eds.), Philosophies of social science (pp.400-405). Maidenhead: McGrawHill.

Gilburt, H. (2013). Promoting recovery-oriented practice in mental health services: A quasiexperimental mixed-methods study. BMC Psychiatry, 13(1), 167.

Gilburt, H. (2015). Mental Health Under Pressure - Kings Fund. London: The King's Fund.

Gilburt, H., Peck, E., Ashton, B., Edwards, N. \& Naylor, C. (2014) Service transformation: Lessons from mental health. London: The Kings Fund.

Glasby, J. and Tew, J. (2015). Mental health policy and practice (3rd ed.). London: Palgrave Macmillan.

Glaser, B. G. and Strauss, A. L. (1967). The Discovery of Grounded Theory: Strategies for Qualitative Research. London: Aldine Transaction.

Gofal. (2013). Snapshot 2: People's experiences of primary mental health care support services in Wales. Bridgend: Gofal.

Gofal. (2014). Snapshot 3: People's experiences of primary mental health care support services in Wales. Bridgend: Gofal.

Gofal. (2015). Snapshot 4: People's experiences of primary mental health services in Wales - Three years on. Bridgend: Gofal.

Goffman, E. (1955). On face work. Psychiatry, 18: 213-31.

Goffman, E. (1959). The presentation of self in everyday life. New York: Doubleday.

Goffman, E. (1971). Relations in public. Harmondsworth: Penguin.

Gordon, J. S. (1990). Holistic medicine and mental health practice: Toward a new synthesis. American Journal of Orthopsychiatry, 60(3), 357-371. 
Gramsci, A. (1971). Selections from the Prison Notebooks of Antonio Gramsci, trans. Q. Hoare and G.N. Smith (eds.). London: Lawrence \& Wishart.

Grant, M. J. and Booth, A. (2009). A typology of reviews: an analysis of 14 review types and associated methodologies. Health Information \& Libraries Journal, 26(2), 91-108.

Green, J. and Thorogood, N. (1998). Analysing health policy: A sociological approach. London: Longman.

Greenhalgh, T. and Peacock, R. (2005). Effectiveness and efficiency of search methods in systematic reviews of complex evidence: audit of primary sources. British Medical Journal, 315, 672-675.

Greenhalgh, T., Plsek, P., Wilson, T., Fraser, S. \& Holt, T. (2010). Response to 'The appropriation of complexity theory in health care'. Journal of Health Services Research \& Policy, 15(2), 115-117.

Grondin, J. (2003). The philosophy of Gadamer, trans. K. Plant. Chesham: Acumen.

Guba, E. G. and Lincoln, Y. S. (1994). 'Competing paradigms in qualitative research' in N. K. Denzin and Y. S. Lincoln (eds.), Handbook of qualitative research (pp.163-194). London: SAGE.

Gubrium, J. F. and Holstein, J. A. (2004). 'The active interview' in D. Silverman (ed.), Qualitative research (2nd ed.) (pp.140-161). London: SAGE.

Gulliford, M., Naithani, S. \& Morgan, M. (2006). What is 'continuity of care'? Journal of Health Service Research \& Policy, 11, 248-250.

Habermas, J. (1970a). On systematically distorted communication. Inquiry, 13(1-4), 205-218.

Habermas, J. (1970b). Towards a theory of communicative competence. Inquiry, 13(1-4), 360-375.

Habermas, J. (1984a). The theory of communicative action: Vol. 1. Reason and the rationalization of society, trans. T. McCarthy. Boston: Beacon Press.

Habermas, J. (1984b). The theory of communicative action: Vol. 2. Lifeworld and system: a critique of functionalist reason, trans. T. McCarthy. Boston: Beacon Press.

Habermas, J. (1991). The structural transformation of the public sphere: An inquiry into a category of bourgeois society. Cambridge: MIT press.

Habermas, J. (2010 [1964]). 'The public sphere: an encyclopedia article' in M. G. Durham and D. M. Kellner (eds.), Media and cultural studies: key works (rev. ed.). Oxford: Blackwell.

Hacking, I. (2003). 'What is social construction? The teenage pregnancy example' in G. Delanty and P. Strydom (eds.), Philosophies of social science: the classic and contemporary readings (pp.421-427). Maidenhead: McGraw-Hill.

Hafal (2012). Hafal - CTP and recovery promotional leaflet, viewed 10 July 2018, http://www.hafal.org/pdf/publications/My\%20recovery.pdf

Haggerty, J. L., Reid, R. J., Freeman, G. K., Starfield, B. H., Adair, C. E. \& McKendry, R. (2003). Continuity of care: a multidisciplinary review. British Medical Journal, 327(7425), 1219-1221. 
Håland, E., Røsstad, T. \& Osmundsen, T. C. (2015). Care pathways as boundary objects between primary and secondary care: Experiences from Norwegian home care services. Health, 19(6), 635-651. doi: http://dx.doi.org/10.1177/1363459314567789

Hall, A., Wren, M. \& Kirby, S. (2013). Care planning in mental health: Promoting recovery $\left(2^{\text {nd }} e d.\right)$. Chichester, West Sussex: John Wiley \& Sons.

Halliday, M. A. K. and Hasan, R. (2014). Cohesion in english. London: Routledge.

Hammersley, M. (2008). 'Assessing the radical critique of interviews' in M. Hammersley (ed.), Questioning qualitative inquiry: critical essays (pp.89-100). Los Angeles, California: SAGE.

Hannigan, B. (2013). Connections and consequences in complex systems: insights from a case study of the emergence and local impact of crisis resolution and home treatment services. Social Science and Medicine, 93, 212-219.

Hannigan, B. and Allen, D. (2013). Complex caring trajectories in community mental health: contingencies, divisions of labor and care coordination. Community Mental Health Journal, 49(4), 380-388. doi: http://dx.doi.org/10.1016/S0277-9536(02)00375-1

Hannigan, B. and Coffey, M. (2011). Where the wicked problems are: the case of mental health. Health Policy, 101(3), 220-227.

Hannigan, B., Simpson, A., Coffey, M., Barlow, S. \& Jones, A. (2018). Care coordination as imagined, care coordination as done: findings from a cross-national mental health systems study. International Journal of Integrated Care, 18(3).

Harper, D. and Speed, E. (2012). Uncovering recovery: The resistible rise of recovery and resilience. Studies in Social Justice, 6(1), 9-n/a.

Harper, L. and McKeown, M. (2018). Why make the effort? Exploring recovery college engagement. Mental Health and Social Inclusion, 22(1), 27-33.

Harrington, A. (2005). Modern social theory: an introduction. Oxford: Oxford University Press.

Harvey, D. (2005). A brief history of neoliberalism. Oxford: Oxford University Press.

Health and Social Care Act. (2012). London: Department of Health.

Heaney, P. (2016). 'Mental health patients wait 'years' for treatment', BBC News, 14 April, https://www.bbc.co.uk/news/uk-wales-36038905

Heaton, J. M. (2006). 'From anti-psychiatry to critical psychiatry' in D. B. Double (ed.), Critical Psychiatry: the limits of madness (pp.41-60). New York: Palgrave Macmillan.

Heaton, J., Corden, A. \& Parker, G. (2012). Continuity of care: a critical interpretive synthesis of how the concept was elaborated by a national research programme. International Journal of Integrated Care, 12: e12. 
Heidegger, M., Macquarrie, J. \& Robinson, E. S. (1967). Being and Time, trans. J. Macquarrie and E. Robinson. Oxford: Blackwell Publishers.

Hewitt, J. (2007). Ethical components of researcher-researched relationships in qualitative interviewing. Qualitative Health Research, 17(8), 1149-1159.

Heywood, A. (2007). Political ideologies and introduction (4th ed.). Basingstoke: Palgrave Macmillan.

HLWIKI International: The University of British Colombia. (2016). Scoping reviews, viewed 29 August 2016, http://hlwiki.slais.ubc.ca/index.php/Scoping_reviews

HM Government. (2011). No health without mental health: a cross-government mental health outcomes strategy for people of all ages. Supporting document: the economic case for improving efficiency and quality in mental health. London: DoH.

https://assets.publishing.service.gov.uk/government/uploads/system/uploads/attachment_data/fi le/215808/dh_123993.pdf

Hodge, B. and Kress, G. R. (1988). Social semiotics. Ithaca, N.Y.: Cornell University Press.

Holstein, J. A. and Gubrium, J. F. (2004). 'The active interview' in D. Silverman (ed.), Qualitative research (2nd ed.) (pp.140-161). London: SAGE.

Holub, R. C. (1991). Jurgen Habermas: Critic in the public sphere. London: Routledge.

Hooker, C. (2011). 'Introduction to philosophy of complex systems: A: Part A: Towards a framework for complex systems' in C. Hooker (ed.), Vol. 10: Philosophy of complex systems (pp.3-90). North-Holland. Boston: Elsevier/Academic.

Hopper, K. (2007). Rethinking social recovery in schizophrenia: what a capabilities approach might offer. Social Science \& Medicine, 65(5), 868-879.

Hopper, K. (2008). A confusion of tongues, a reform on hold. Chronic illness, 4(4), 307-308.

Hopton, J. (2006). The Future of Critical Psychiatry. Critical Social Policy, 26(1), 57-73. doi: http://dx.doi.org/10.1177/0261018306059776

Houston, S. (2001). Beyond social constructionism: Critical realism and social work. British Journal of Social Work, 31(6), 845-861.

Humphries, R. and Curry, N. (2011). Integrating health and social care: where next? London: The King's Fund.

Hyman, S. E. (2010). The diagnosis of mental disorders: the problem of reification. Annual Review of Clinical Psychology, 6, 155-179.

Illich, I. (1993). 'The epidemics of modern medicine' in N. Black, D. Boswell, A. Gray, S. Murphy \& J. Popay (eds.), Health and disease: a reader. Milton Keynes: Open University Press.

Ingleby, D. (2006). 'Transcultural mental health care: The challenge to positivist psychiatry' in D. B. Double (ed.), Critical psychiatry: the limits of madness (pp.61-78). New York: Palgrave Macmillan. 
Jabareen Y. (2009). Building a conceptual framework: philosophy, definitions, and procedure. International Journal of Qualitative Methods, 8, 49-62.

Jacobson, N. and Greenley, D. (2001). What is recovery? A conceptual model and explication. Psychiatric Services, 52(4), 482-485.

Jaspers K. (1997 [1913]). General Psychopathology, trans. J. Hoenig and M. W. Hamilton. Baltimore, MD: Johns Hopkins University.

Jefferson, G. (1984). 'On the organization of laughter in talk about troubles' in J. M. Atkinson and J. Heritage (eds.), Structures of social action: studies in conversation analysis (pp.346-369). Cambridge: Cambridge University Press.

Jensen, N. K., Johansen, K. S., Kastrup, M., Krasnik, A. \& Norredam, M. (2014). Patient experienced continuity of care in the psychiatric healthcare system-a study including immigrants, refugees and ethnic danes. International Journal of Environmental Research and Public Health, 11(9), 9739-9759. doi: http://dx.doi.org/10.3390/ijerph110909739

Jingree, T. and Finlay, W. (2013). Expressions of dissatisfaction and complaint by people with learning disabilities: A discourse analytic study. British Journal of Social Psychology, 52(2), 255-272.

Johnson, C., Aaronson, N., Blazeby, J. M., Bottomley, A., Fayers, P., Koller, M., Kulis, D., Ramage, J., Sprangers, M., Velikova, G. \& Young, T. (2011). EORTC quality of life group: Guidelines for developing questionnaire modules ( $4^{\text {th }}$ ed.). Brussels: EORTC.

Johnson, C., Fitzsimmons, D., Gilbert, J., Arrarras, J., Hammerlid, E., Bredart, A., Ozmen, M., Dilektasli, E., Coolbrandt, A., Kenis, C., Yound, T., Chow, E., Ramachandran, V., Howse, F., George, S., O’Connor, S. \& Yadegarfar, G. (2010). Development of the European organisation for research and treatment of cancer quality of life questionnaire module for older people with cancer: The EORTC QLQ-ELD15. European Journal of Cancer, 46, 2242-2252.

Johnson, S., Prosser, D., Bindman, J. \& Szmukler, G. (1997). Continuity of care for the severely mentally ill: concepts and measures. Social Psychiatry and Psychiatric Epidemiology, 32(3), 137-142.

Jørgensen, M. W. and Phillips, L. J. (2002). Discourse analysis as theory and method. London: SAGE. Joyce, A. S., Adair, C. E., Wild, T. C., McDougall, G. M., Gordon, A., Costigan, N. \& Pasmeny, G. (2010). Continuity of care: validation of a self-report measure to assess client perceptions of mental health service delivery. Community Mental Health Journal, 46(2), 192-208. doi: http://dx.doi.org/10.1007/s10597-009-9215-6

Joyce, A. S., Wild, T. C., Adair, C. E., McDougall, G. M., Gordon, A., Costigan, N., Beckie, A., Kowalsky, L., Pasmeny, G. \& Barnes, F. (2004). Continuity of care in mental health services: toward clarifying the construct. The Canadian Journal of Psychiatry, 49(8), 539-550. 
Kant, I. (1998 [1781]). The critique of pure reason, trans. P. Guyer and A. W. Wood (eds.). Cambridge: Cambridge University Press.

Keetharuth, A. D., Brazier, J., Connell, J., Bjorner, J. B., Carlton, J., Buck, E. T., Ricketts, T., McKendrick, K., Browne, J., Croudace, T. \& Barkham, M. (2018). Recovering Quality of Life (ReQoL): a new generic self-reported outcome measure for use with people experiencing mental health difficulties. The British Journal of Psychiatry, 212(1), 42-49.

Kenny, A. (2006). The rise of modern philosophy. Oxford: Clarendon Press.

Kincheloe, J. L. and McLaren, P. L. (1994). 'Rethinking critical theory and qualitative research' in N. K. Denzin and Y. S. Lincoln (eds.), Handbook of qualitative research (pp.138-157). London: SAGE.

Kirk, J. and Miller, M. L. (1986). Reliability and validity in qualitative research. Newbury Park, CA: SAGE.

Kisby, B. (2010). The Big Society: power to the people? The Political Quarterly, 81(4), 484-491.

Kitzinger, C. (2004). 'Feminist approaches' in T. J. Servoss, W. Government, J. F. Gubrium, \& D. Silverman (eds.), Qualitative research practice (pp.125-140). London: SAGE.

Kristeva, J. (1986). 'Word, dialogue and novel' in T. Moi (ed.), The Kristeva reader (pp.34-61). Oxford: Basil Blackwell.

Kuhn, T. S. (2012). The structure of scientific revolutions (4th. ed.). London: The University of Chicago Press.

Laclau, E. and Mouffe, C. (1985). Hegemony and socialist strategy. London: Verso.

Lakoff, G. (1973). Hedges: A study in meaning criteria and the logic of fuzzy concepts. Journal of Philosophical Logic, 2(4), 458-508.

Lal, S. (2010). Prescribing recovery as the new mantra for mental health: Does one prescription serve all? Canadian Journal of Occupational Therapy, 77(2), 82-89.

Lapadat, J. C. and Lindsay, A. C. (1999). Transcription in research and practice: from standardization of technique to interpretive positionings. Qualitative Inquiry, 5, 64-86.

Layder, D. (2005). Modern social theory: Key debates and new directions. London: Routledge.

Leamy, M., Bird, V., Le Boutillier, C., Williams, J. \& Slade, M. (2011). Conceptual framework for personal recovery in mental health: systematic review and narrative synthesis. The British Journal of Psychiatry, 199(6), 445-452.

LeComte, M. D. and Goetz, J. P. (1982). Problems of reliability and validity in ethnographic research. Review of Educational Research, 52, 31-60.

Lester, H. (2006). Current issues in providing primary medical care to people with serious mental illness. The International Journal of Psychiatry in Medicine, 36(1), 1-12. 
Lester, H. and Gask, L. (2006). Delivering medical care for patients with serious mental illness or promoting a collaborative model of recovery? British Journal of Psychiatry, 188, 401-402.

Lester, H., Glasby, J. \& Tylee, A. (2004). Integrated primary mental health care: threat or opportunity in the new NHS? British Journal of General Practice, 54(501), 285-291.

Lester, H. E., Tritter, J. Q. \& Sorohan, H. (2005). Patients' and health professionals' views on primary care for people with serious mental illness: focus group study. British Medical Journal, $330(7500), 1122$.

Lincoln, Y. S. and Guba, G. E. (1985). Naturalistic inquiry. Beverley Hills, CA: SAGE.

Lindquist, R., Tracy, M. F. \& Snyder, M. (2018). Complementary and alternative therapies in nursing. New York: Springer Publishing Company.

Lipsky, M. (1980). Street-Level Bureaucracy: Dilemmas of the Individual in Public Service. New York: Russell Sage Foundation.

Locke, T. (2004). Critical discourse analysis. New York: Continuum.

Lorig, K. R., Mazonson, P. D. \& Holman, H. R. (1993). Evidence suggesting that health education for self-management in patients with chronic arthritis has sustained health benefits while reducing health care costs. Arthritis \& Rheumatism: Official Journal of the American College of Rheumatology, 36(4), 439-446.

Lucas, P. J., Arai, L., Baird, L. C. \& Roberts, H. M. (2007). Worked examples of alternative methods for the synthesis of qualitative and quantitative research in systematic reviews. BMC Medical Research Methodology, 7, 4.

Luhmann, N. (1985). A Sociological Theory of Law. London: Routledge and Kegan Paul.

Luhmann, N. (1995). Social systems. Stanford: Stanford University Press.

Luhmann, N. (1996). On the scientific context of the concept of communication. Social Science Information, 35(2), 257-267.

Luhmann, N. (2012a). Theory of Society, Volume 1. Stanford: Stanford University Press.

Luhmann, N. (2012b). Theory of Society, Volume 2. Stanford: Stanford University Press.

Lyman, S. M. and Scott, M. B. (1970). A sociology of the absurd. New York: Appleton-Century-Crofts.

Lynch, M. (2000). Against reflexivity as an academic virtue and source of privileged knowledge.

Theory, Culture and Society, 17, 26-54.

Lyotard, J. F. (1984). The Postmodern Condition: A Report on Knowledge. Manchester: Manchester University Press.

Lyotard, J. F. (1991). Phenomenology. Albany: State University of New York Press.

MacGregor, S. (2005). 'The welfare state and neoliberalism' in A. Saad-Filho and D. Johnston (eds.), Neoliberalism: a critical reader (pp.142-148). London: Pluto Press. 
Manicas, P. T. (2006). A realist philosophy of social science explanation and understanding. Cambridge: Cambridge University Press.

Maturana, H. R. and Varela, F. J. (1980). Autopoiesis and cognition: the realization of the living. London: D. Reidel.

Maund, D. (2012). Conserving the left: the 'left conservative' critique of postmodernism and postMarxism - an intellectual encounter. Doctoral thesis, University of Western Australia. https://research-repository.uwa.edu.au/files/3221339/Maund_Dolores_2012.pdf

May, T. and Perry, B. (2013). 'Reflexivity and the Practice of Qualitative Research' in U. Flick (ed.), The SAGE handbook of qualitative data analysis (pp.109-122). London: SAGE.

Mays, N., Roberts, E. \& Popay, J. (2001). 'Synthesising research evidence' in N. Fulop, P. Allen, A. Clarke and N. Black (eds.), Studying the organisation and delivery of health services: research methods (pp.188-220). London: Routledge.

McCarthy, T. (1984). 'Translator's introduction' in J. Habermas, The theory of communicative action: Vol. 1. Reason and the rationalization of society (pp.vii-XXXiX). Boston: Beacon Press.

McClelland, K., and Thomas J. (eds.). (2006). Purpose, meaning, and action: control systems theories in sociology. New York: Palgrave Macmillan.

McCranie, A. (2010). 'Recovery in mental illness: the roots, meanings, and implementations of a 'new'services movement' in D. Pilgrim, A. Rogers \& B. A. Pescosolido, The SAGE handbook of mental health and illness (pp.471-489). London: SAGE.

McGuire-Snieckus, R., McCabe, R., Catty, J., Hansson, L. \& Priebe, S. (2007). A new scale to assess the therapeutic relationship in community mental health care: STAR. Psychological Medicine, 37(1), 85-95.

McKeith, J. and Burns, S. (2010). The Recovery Star: User Guide (2 ${ }^{\text {nd }}$ ed.). London: Mental Health Providers Forum. www.mhpf.org.uk

McKeown, M., Wright, K. \& Mercer, D. (2017). Care planning: a neoliberal three card trick. Journal of Psychiatric and Mental Health Nursing, 24(6), 451-460.

McLeod, J. (2001). Qualitative research in counselling and psychotherapy. London: SAGE.

McNicoll A. (2013). 'We are fire-fighting': the mental health funding shortage that's hitting frontline care', Community Care, 12 December, https://www.communitycare.co.uk/2013/12/12/firefighting-mental-health-funding-shortagethats-hitting-frontline-care/

McNicoll, A. (2015). 'Mental health trust funding down 8\% from 2010 despite coalition's drive for parity of esteem', Community care, 20 March, https://www.communitycare.co.uk/2015/03/20/mental-health-trust-funding-8-since-2010despite-coalitions-drive-parity-esteem/ 
Mead, G. H. (1934). Mind, Self and Society. Chicago, IL: Chicago University Press.

Mental Capacity Act. (2005). London: Department of Health.

Merleau-Ponty, M. (2013 [1962]). Phenomenology of perception. London: Routledge.

Middleton, H. (2007). Critical Psychiatry. Mental Health Review Journal, 12(2), 40-44. doi: http://dx.doi.org/10.1108/13619322200700021

Miller, R. L. and Brewer, J. D. (eds.) (2003). The A-Z of social research: a dictionary of key social science research concepts. London: SAGE.

Mind. (2008). Life and times of a supermodel. The recovery paradigm for mental health. MindThink report 3, viewed 12 September 2018, https://www.bl.uk/britishlibrary/ /media/bl/global/socialwelfare/pdfs/non-secure/1/i/f/life-and-times-of-a-supermodel-the-recovery-paradigm-formental-health-001.pdf

Mishler, E. G. (2005). Patient stories, narratives of resistance and the ethics of humane care: A la recherche du temps perdu. Health: An Interdisciplinary Journal for the Social Study of Health, Illness and Medicine, 9(4), 431-452. doi: http://dx.doi.org/10.1177/1363459305056412

Mitchell, J. C. (1983). Case and situation analysis. The Sociological Review, 31(2), 187-211.

Mokkink, L. B., Terwee, C. B., Patrick, D. L., Alonso, J., Stratford, P. W., Knol, D. L., Bouter, L. M. \& de Vet, H. C. (2012). COSMIN checklist manual. Amsterdam: University Medical Center.

Moncrieff, J. (2006). 'The politics of psychiatric drug treatment' in D. B. Double (ed.), Critical psychiatry: The limits of madness (pp.115-132). New York: Palgrave Macmillan.

Moncrieff, J. (2009). The myth of the chemical cure: a critique of psychiatric drug treatment (rev. ed.). Basingstoke: Palgrave Macmillan.

Montori, V., Wilczynski, N., Morgan, D. \& Haynes, R. (2004). Optimal search strategies for retrieving systematic reviews from Medline: an analytic surney. British Medical Journal, 330, 7482.

Morrow, M. (2013). 'Recovery: Progressive paradigm or neoliberal smokescreen?' in B. A. LeFrancois, R. Menzies \& G. Reaume (eds.), Mad matters: a critical reader in Canadian mad studies (pp.323-333). Toronto: Canadian Scholars' Press.

Mulhall, S. (1996). Routledge philosophy guidebook to Heidegger and Being and Time. London: Routledge.

Nettleton, S. (2013). The Sociology of Health and Illness (3rd ed.). Cambridge: Polity Press.

NHS and Community Care Act. (1990). London: Department of Health.

NHS Wales. (2018). The quality of care and treatment planning assurance review of adult mental health \& learning disability services national report, viewed 7 August 2019, http://www.wales.nhs.uk/sitesplus/863/opendoc/335464

NIMHE. (2005). NIMHE guiding statement on recovery, viewed 14 July 2018, http://studymore.org.uk/nimherec.pdf 
North, N. and Peckham, S. (2001). Analysing structural interests in primary care groups. Social Policy \& Administration, 35(4), 426-440.

Noy, C. (2008). Sampling knowledge: the hermeneutics of snowball sampling in qualitative research. International Journal of Social Research Methodology, 11, 327-44.

O'Connor, S. (1992). Network theory - a systematic method for literature review. Nurse Education Today, 12, 44-50.

O’Keeffe, D., Sheridan, A., Kelly, A., Doyle, R., Madigan, K., Lawlor, E. \& Clarke, M. (2018). 'Recovery'in the real world: service user experiences of mental health service use and recommendations for change 20 years on from a first episode psychosis. Administration and Policy in Mental Health and Mental Health Services Research, 45(4), 635-648.

OECD: Organization for Economic Cooperation and Development. (2018). Health at a glance: Europe 2018. Paris: Organisation for Economic Cooperation and Development Publishing.

Orbuch, T. L. (1997). People's accounts count: The sociology of accounts. Annual Review of Sociology, 23(1), 455-478.

ORS: Opinion Research Services. (2014). Scoping Study Report - Research to Support the Duty to Review the Implementation of the Mental Health (Wales) Measure 2010. Welsh Government: Cardiff.

Osterberg, D. (2000). Luhman's general sociology. Acta Sociologica, 43, 15-25.

Palley, T.I. (2005). 'From Keynesianism to neoliberalism: shifting paradigms in economics' in A. SaadFilho and D. Johnston (eds.), Neoliberalism: a critical reader (pp.20-29). London: Pluto Press.

Panella, M., Van Zelm, R., Sermeus, W. \& Vanhaecht, K. (2012). Care pathways for the organization of patients' care. Bulletin: Economics, Organisation and Informatics in Healthcare, 28(2), 111122 .

Parsons, T. (1951a). The Social System. Glencoe, IL: Free Press.

Parsons, T. (1951b). Illness and the role of the physician: A sociological perspective. American Journal of Orthopsychiatry, 21(3), 452-460.

Parsons, T. (1961). An Outline of the Social System. University of Puerto Rico, Department of Social Sciences.

Patterson, E. F. (1998) The philosophy and physics of holistic health care: spiritual healing as a workable interpretation. Journal of Advanced Nursing 27, 287-93.

Perkins, R. and Repper, J. (2018). Thinking about recovery and well-being in a social context. Mental Health and Social Inclusion, 22(4), 161-166.

Perkins, R., Repper, J., Rinaldi, M. \& Brown, H. (2012). Recovery Colleges. London: Centre for Mental Health.

Phillips, D. C. (1977). Holistic thought in social science. London: Macmillan. 
Pickel, A. (2011). 'Systems Theory' in I. C. Jarvie and J. Zamora-Bonilla (eds.), The SAGE handbook of the philosophy of social sciences (pp.240-251). London: SAGE.

Pilgrim, D. (2008). Recovery and current mental health policy. Chronic Illness, 4(4), 295-304.

Pilgrim, D. and McCranie, A. (2013). Recovery and mental health: A critical sociological account. New York: Palgrave Macmillan.

Plsek, P. E. and Greenhalgh, T. (2001). The challenge of complexity in health care. British Medical Journal, 323(7313), 625.

Polit, D. F. and Beck, C. T. (2010). Generalization in quantitative and qualitative research: Myths and strategies. International Journal of Nursing Studies, 47(11), 1451-1458.

Pomerantz, A. (1984). 'Agreeing and disagreeing with assessments: Some featurres of preferred/dsipreferred turn shapes' in J. Atkinson and J. Heritage (eds.), Structures of social action (pp.57-101). Cambridge: Cambbridge University Press.

Pomerantz, A. (1986). Extreme case formulations: A way of legitimizing claims. Human Studies, 9(23), 219-229.

Poremski, D., Harris, D.W., Kahan, D., Pauly, D., Leszcz, M., O'Campo, P., Wasylenki, D. \& Stergiopoulos, V. (2016). Improving continuity of care for frequent users of emergency departments: service user and provider perspectives. General Hospital Psychiatry, 40, 55-59. doi: http://dx.doi.org/10.1016/j.genhosppsych.2016.01.004

Porter, S. (1998). Social theory and nursing practice. London: Macmillan Press.

Potter, J. (1996). 'Discourse analysis and constructionist approaches: theoretical background' in J. T. E. Richardson (ed), Handbook of qualitative research methods for psychology and the social sciences (pp.125-140). Leicester: British Psychological Society.

Potter, J. (1997). 'Discourse analysis as a way of analysing naturally occurring talk' in D. Silverman (ed.), Qualitative research: theory, method and practice (pp.200-222). London: SAGE.

Potter, J. (2003). 'Discourse analysis and discursive psychology' in H. Cooper (ed.), APA handbook of research methods in psychology: Vol. 2. Quantitative, qualitative, neuropsychological and biological. Washington: American Psychological Association Press.

Potter, J. and Wetherell, M. (1987). Discourse and social psychology: Beyond attitudes and behaviour. London: SAGE.

Pressman, J. and Wildavsky, A. (1973). Implementation. Berkeley, CA: University of California Press. Protevi, J. (2005). Edinburgh dictionary of continental philosophy. Edinburgh: Edinburgh University Press.

Provencher, H., Gregg, R., Mead, S. \& Mueser, K. (2002). The role of work in the recovery of persons with psychiatric disabilities, Psychiatric Rehabilitation Journal, 26(2), 132-144. 
Puntis, S., Rugkasa, J., Forrest, A., Mitchell, A. \& Burns, T. (2014). Associations between continuity of care and patient outcomes in mental health care: a systematic review. Psychiatric Services, 66(4), 354-363. doi: http://dx.doi.org/10.1176/appi.ps.201400178

Pusey, M. (2002). Jürgen Habermas. London: Routledge.

Putnam, R. D. (1995). Tuning in, tuning out: the strange disappearance of social capital in America. Political Science and Politics 28, 664-83.

Ramanuj, P. P., Carvalho, C. F., Harland, R., Garety, P. A., Craig, T. K. \& Byrne, N. (2015). Acute mental health service use by patients with severe mental illness after discharge to primary care in South London. Journal of Mental Health, 24(4), 208-213.

Rapley, T. (2004). 'Interviews' in J. Searle, G. Gobo, J. F. Gubrium, \& D. Silverman (eds.), Qualitative research practice (pp.15-33). London: SAGE.

RCPsych: The Royal College of Psychiatrists. (2014). Post-legislative Scrutiny of the Mental Health (Wales) Measure 2010. Cardiff: Welsh Government.

RCPsych: The Royal College of Psychiatrists. (2018). CR207: College Report: Safe patients and highquality services. Job descriptions for consultant psychiatrists. London: Royal College of Psychiatrists.

Recovery in the Bin. (2019). Neorecovery: a survivor led conceptualisation And critique. In Proceedings of the International Mental Health Nursing Research Conference. RCN Headquarters, London.

Repper, J. and Perkins, R. (2003). Social inclusion and recovery: a model for mental health practice. London: Elsevier Limited.

Rhodes, P. and Giles, S. J. (2014). 'Risky Business': a critical analysis of the role of crisis resolution and home treatment teams. Journal of Mental Health, 23, 130-4.

Richards, H. M. and Schwartz, L. J. (2002). Ethics of qualitative research: are there special issues for health services research? Family Practice 19, 135-139.

Richter, R. R. and Austin, T. M. (2012). Using MeSH (Medical Subject Headings) to enhance PubMed search strategies for evidence-based practice in physical therapy. Physical Therapy, 92(1), 124132.

Riessman, C. K. (1993). Narrative analysis. Thousand Oaks, CA: SAGE.

Riessman, C. K. (2008). Narrative methods for the human sciences. London: SAGE.

Ritchie, J. and Spencer, L. (1994). 'Qualitative data analysis for applied policy research' in A. Bryman and R. Burgess (eds.), Analysing qualitative data (pp.173-194). London: Routledge.

Ritchie, J. H., Dick, D. \& Lingham, R. (1994). The report of the inquiry into the care and treatment of Christopher Clunis. London: HM Stationery Office. 
Ritchie, J., Lewis, J., Nicholls, C. M. \& Ormston, R. (2013). Qualitative research practice: A guide for social science students and researchers. London: SAGE.

Rittel, H. and Webber, M. (1973). Dilemmas in a general theory of planning. Policy Sciences, 4(2), 155-169. doi: http://dx.doi.org/10.1007/BF01405730

Rivas, C. (2011). 'Coding and analysing qualitative data' in C. Seale (ed.), Researching society and culture (pp.366-392). London: SAGE.

Robertson, R. (2016). Six ways in which NHS financial pressures can affect patient care. London: The King's Fund.

Rogers, A. and Pilgrim, D. (2014). A sociology of mental health and illness (5th ed.). Maidenhead: Open University Press.

Rogers, E. M. (2003). Diffusion of Innovations (5th ed.). New York: Free Press.

Rorty, R. (1980). Philosophy and the mirror of nature. Oxford: Blackwell.

Rorty, R. (1982). Consequences of pragmatism: Essays, 1972-1980. Minnesota: University of Minnesota Press.

Rorty, R. (1985). 'Solidarity or Objectivity' in J. Rajchman and C. West (eds.), Post-analytic philosophy (pp.3-19). New York: Colombia University Press.

Rose, D. (2013). 'Genre in the Sydney school' in J. P. Gee and M. Handford (eds.), The Routledge handbook of discourse analysis (pp.209-225). London: Routledge.

Rose, D. (2014). The mainstreaming of recovery. Journal of Mental Health, 23(5), 217-218. doi: https://doi.org/10.3109/09638237.2014.928406

Rose, D., Sweeney, A., Leese, M., Clement, S., Jones, I.R., Burns, T., Catty, J. \& Wykes, T. (2009). Developing a user-generated measure of continuity of care: brief report. Acta Psychiatrica Scandinavica, 119(4), 320-324. doi: http://dx.doi.org/10.1111/j.1600-0447.2008.01296.x

Rose, D., Thornicroft, G. \& Slade, M. (2006). Who decides what evidence is? Developing a multiple perspectives paradigm in mental health. Acta Psychiatrica Scandinavica, 113, 109-114.

Rudge, T. and Morse, K. (2001). Re-awakenings? A discourse analysis of the recovery from schizophrenia after medication change. The Australian and New Zealand Journal of Mental Health Nursing, 10(2), 66-76.

Rundell, M. and Fox, G. (2010). Macmillan collocations dictionary. London: Macmillan Education.

Ryan, G. W. and Bernard, H. R. (2000). 'Data management and analysis methods' in N. K. Denzin and Y. S. Lincoln (eds.), Handbook of qualitative research (2nd ed.) (pp.769-802). London: SAGE.

SAMHSA: National Consensus Conference on Mental Health Recovery \& Mental Health Systems Transformation. (2004). National Consensus Statement on Mental Health Recovery. Rockville, MD: Substance Abuse and Mental Health Services Administration, U.S. Department of Health and Human Services. 
Saussure, F. (1974). Course in General Linguistics. London: Fontana.

Schrijvers, G., van Hoorn, A. \& Huiskes, N. (2012). The care pathway: concepts and theories: an introduction. International Journal of Integrated Care, 12 (Special Edition Integrated Care Pathways).

Schultz, E. M. and McDonald, K. M. (2014). What is care coordination? International Journal of Care Coordination, 17(1-2), 5-25.

Schutz, A. and Luckmann, T. (1974). The structures of the life-world, trans. R. M. Zaner and H. T. Engelhardt Jr. London: Heinemann.

Schwandt, T. (2000). 'Three epistemological stances for qualitative inquiry: interpretivism, hermeneutics and social constructionism' in N. K. Denzin and Y. S. Lincoln (eds.), Handbook of qualitative research (pp.189-213). London: SAGE.

Schwandt, T. (2006). Opposition redirected. International Journal of Qualitative Studies in Education, 19(6), 803-810. doi: http://dx.doi.org/10.1080/09518390600979323

Scott, M. B. and Lyman, S. M. (1968). Accounts. American Sociological Review, 3(1), 46-62.

Scottish Executive (2006). Delivering for mental health. Edinburgh: Scottish Executive.

Scottish Government. (2001). Adults with Incapacity (Scotland) Act (2000) - Code of Practice for Practitioners Authorised to Carry Out Medical Treatment or Research Under Part 5 of the Act. Edinburgh: Scottish Government.

Scruton, R. (1994). Modern philosophy: a survey. London: Sinclair-Stevenson.

Seale, C. (1999). The quality of qualitative research. Oxford: Blackwell.

Seale, C. (2011). 'Validity, reliability and the quality of research' in C. Seale (ed.), Researching society and culture (pp.528-543). London: SAGE.

Searle, J. R. (1979). Expression and Meaning. Cambridge: Cambridge University Press.

Segre, S. (2014). Contemporary sociological thinkers and theories. Farnham: Ashgate Publishing.

Senker, S. and Green, G. (2016). Understanding recovery: the perspective of substance misusing offenders. Drugs and Alcohol Today, 16(1), 16-28. doi: http://dx.doi.org/10.1108/DAT-07-2015$\underline{0032}$

Shepherd, G., Boardman, J., Rinaldi, M. \& Roberts, G. (2014). Supporting recovery in mental health services: quality and outcomes. London: Implementing Recovery Through Organisational Change, viewed 12 February 2019, http://www.slamrecoverycollege.co.uk/uploads/2/6/5/2/26525995/imroc_08 supporting_recove ry in mental_health_services.pdf

Silverman, D. (2011). 'Research and theory' in C. Seale (ed.), Researching society and culture (pp.2944). London: SAGE.

Silverman, D. (2014). Interpreting qualitative data. London: SAGE. 
Simpson, A. (2005). Community psychiatric nurses and the care co-ordinator role: squeezed to provide 'limited nursing'. Journal of Advanced Nursing, 52(6), 689-699.

Simpson, A., Hannigan, B., Coffey., Jones, A., Barlow, S., Cohen, R., Vseteckova, J., Faulkner, A. \& Haddad, M. (2015). Study protocol: cross-national comparative case study of recovery-focused mental health care planning and coordination (COCAPP). BMC Psychiatry, 15, 145.

Simpson, A., Hannigan, B., Coffey, M., Barlow, S., Cohen, R., Jones, A., Všetečková, J., Faulkner, A., Thornton, A. \& Cartwright, M. (2016a). Recovery-focused care planning and coordination in England and Wales: a cross-national mixed methods comparative case study. BMC Psychiatry, 16(1), 147.

Simpson, A., Hannigan, B., Coffey, M., Jones, A., Barlow, S., Cohen, R., Všetečková, J. \& Faulkner, A. (2016b). Cross-national comparative mixed-methods case study of recovery-focused mental health care planning and co-ordination: Collaborative Care Planning Project (COCAPP). Health Services and Delivery Research, 4(5). doi: http://dx.doi.org/10.3310/hsdr04050

Simpson, A., Miller, C. \& Bowers, L. (2003). Case management models and the care programme approach: how to make the CPA effective and credible. Journal of Psychiatric and Mental Health Nursing, 10(4), 472-483.

Simpson, A., Miller, C. \& Bowers, L. (2003). The history of the Care Programme Approach in England: Where did it go wrong? Journal of Mental Health, 12(5), 489-504.

Singer, D. and Hunter, M. (1999). The experience of premature menopause: a thematic discourse analysis. Journal of Reproductive and Infant Psychology, 17, 63-81.

Slade, M. (2009). Personal recovery and mental illness: A guide for mental health professionals. Cambridge: Cambridge University Press.

Slade, M., Amering, M., Farkas, M., Hamilton, B., O'Hagan, M., Panther, G., Perkins, R., Shepherd, G., Tse, S. \& Whitley, R. (2014). Uses and abuses of recovery: implementing recovery-oriented practices in mental health systems. World Psychiatry, 13(1), 12-20.

Smaling, A. (2003). Inductive, analogical, and communicative generalization. International Journal of Qualitative Methods, 2(1), 52-67.

Smith, Adam. (2001 [1776]). An inquiry into the nature and causes of the wealth of nations. London: Electric Book.

Smith-Merry, J., Freeman, R. \& Sturdy, S. (2011). Implementing recovery: an analysis of the key technologies in Scotland. International Journal of Mental Health Systems, 5(1), 11.

Spandler, H. and McKeown, M. (2017). Exploring the case for truth and reconciliation in mental health services. Mental Health Review Journal, 22(2), 83-94.

Spradley, J. P. (1979). The ethnographic interview. New York: Holt, Rinehart and Winston. 
Stanghellini, G., Bolton, D. \& Fulford, W. K. (2013). Person-centered psychopathology of schizophrenia: building on Karl Jaspers' understanding of patient's attitude toward his illness. Schizophrenia Bulletin, 39(2), 287-294. doi: http://dx.doi.org/10.1093/schbul/sbs154

Strauss, A. L. (1985). Social organization of medical work. London, IL: University of Chicago Press.

Strauss, A. L. (1987). Qualitative analysis for social scientists. Cambridge: Cambridge University Press.

Strauss, A. L. and Corbin, J. (1990). Basics of qualitative research. Thousand Oaks, CA: SAGE.

Streiner, D. L., Norman, G. R. \& Cairney, J. (2015). Health measurement scales: a practical guide to their development and use ( $5^{\text {th }}$ ed.). Oxford: Oxford University Press.

Sullivan, M. (2003). The new subjective medicine: taking the patient's point of view on health care and health. Social Science \& Medicine, 56, 1595-1604.

Sweeney, A., Davies, J., McLaren, S., Whittock, M., Lemma, F., Belling, R., Clement, S., Burns, T., Catty, J., Jones, I.R. \& Rose, D. (2016). Defining continuity of care from the perspectives of mental health service users and professionals: an exploratory, comparative study. Health Expectations, 19(4), 973-987.

Sweeney, A., Rose, D., Clement, S., Jichi, F., Jones, I. R., Burns, T., Catty, J., Mclaren, S. \& Wykes, T. (2012). Understanding service user-defined continuity of care and its relationship to health and social measures: a cross-sectional study. BMC Health Services Research, 12(1), 145.

Szasz, T. S. (1974a). The myth of mental illness : foundations of a theory of personal conduct (rev. ed.). London : Harper \& Row.

Szasz, T. S. (1974b). Ideology and insanity. Harmondsworth: Penguin Books.

Taylor, C. (1993). 'Engaged Agency and Background in Heidegger' in C. Guignon (ed.), The Cambridge companion to Heidegger (pp.317-336). Cambridge: Cambridge University Press.

Terry, J. (2018). Talking about mental health nursing: a qualitative analysis of nurses' and service users' accounts. Doctoral thesis, Swansea University. http://cronfa.swan.ac.uk/Record/cronfa43230

Tesch, R. (1990). Qualitative research: Analysis types and software tools. London: Falmer.

Tew, J., Ramon, S., Slade, M., Bird, V., Melton, J. \& Le Boutillier, C. (2011). Social Factors and Recovery from Mental Health Difficulties: A Review of the Evidence. British Journal of Social Work 42(3), 443-460.

Thielke, S., Vannoy, S. \& Unutzer, J. (2007). Integrating mental health and primary care. Primary Care, 34(3), 571-592. doi: http://dx.doi.org/10.1016/j.pop.2007.05.007

Thomas, B. H., Ciliska, D., Dobbins, M. \& Micucci, S. (2004). A process for systematically reviewing the literature: providing the research evidence for public health nursing interventions. 
Worldviews Evidence Based Nursing, 1(3), 176-184. doi: http://dx.doi.org/10.1111/j.1524475X.2004.04006.X

Thomas, P. (2016). Psycho politics, neoliberal governmentality and austerity. Self \& Society, 44(4), 382-393.

Thomas, P. and Morris, D. (2016). Integrating primary mental health care and mental health promotion. British Journal of General Practice, 60-61.

Thompson, C. J. and Troester, M. (2002). Consumer value systems in the age of postmodern fragmentation: The case of the natural health microculture. Journal of Consumer Research, 28(4), 550-571.

Tiemans, B., Ormel, J. \& Simon, G. (1996). Occurrence recognition and outcome of psychological disorders in primary care. Psychological Medicine, 153, 636-644.

Tomita, A. and Herman, D. B. (2015). The role of a critical time intervention on the experience of continuity of care among persons with severe mental illness after hospital discharge. The Journal of Nervous and Mental Disease, 203(1), 65-70.

Tracy, S. J. (2010). Qualitative quality: Eight a"big-tent" criteria for excellent qualitative research. Qualitative Inquiry, 16(10), 837-851.

Tricco, A. C., Lillie, E., Zarin, W., O'Brien, K. K., Colquhoun, H., Levac, D., Moher, D., Peters, M. D., Horsley, T., Weeks, L. \& Hempel, S. (2018). PRISMA extension for scoping reviews (PRISMA$\mathrm{ScR}$ ): checklist and explanation. Annals of Internal Medicine, 169(7), 467-473.

Tucker, B. R. and Swartz, C. L. (1926). Individual Liberty. New York: Vanguard Press.

Uijen, A. A., Heinst, C., Schellevis, F., Bosch, W., Laar, F., Terwee, C. \& Schers, H. (2012). Measurement of properties of questionnaires measuring continuity of care: A systematic review. PLoS ONE 7(7), e42256.

Uijen, A. A., Schers, H. J., Schene, A. H., Schellevis, F. G., Lucassen, P. \& van den Bosch, W. J. H. M. (2014). Experienced continuity of care in patients at risk for depression in primary care. The European Journal of General Practice, 20(3), 161-166. doi: http://dx.doi.org/10.3109/13814788.2013.828201

Van Dijk, C. E., Korevaar, J. C., Koopmans, B., de Jong, J. D. \& de Bakker, D. H. (2014). The primary-secondary care interface: Does provision of more services in primary care reduce referrals to medical specialists? Health Policy, 118(1), 48-55. doi:

http://dx.doi.org/10.1016/j.healthpol.2014.04.001

Van Leeuwen, T. and Wodak, R. (1999). Legitimizing immigration control: a discourse historical analysis. Discourse Studies 1(1): 83-118.

Ventegodt S., Kandel I., Ervin D. A. \& Merrick J. (2016). 'Concepts of Holistic Care' in I. L. Rubin, J. Merrick, D. E. Greydanus \& D. R. Patel (eds.), Health care for people with intellectual and 
developmental disabilities across the lifespan (pp.1935-1941). New York: Springer Publishing Company.

Von Foerster, H. (1981). Observing systems. Seaside, CA: Intersystems Publications.

Von Foerster, H. and Zopf, G. (eds.) (1962). Principles of self-organisation. New York: Pergamon.

Walby, S. (2007). Complexity Theory, Systems Theory, and Multiple Intersecting Social Inequalities. Philosophy of the Social Sciences, 37(4), 449. doi: http://dx.doi.org/10.1177/0048393107307663

Walker, M. T. (2006). The social construction of mental illness and its implications for the recovery model. International Journal of Psychosocial Rehabilitation, 10(1), 71-87.

WaMH in PC: Wales Mental Health in Primary Care Network. (2015). Experiences of Delivering Primary Mental Health Care. (2015). Cardiff: Royal College of General Practitioners.

Ware, N. C., Dickey, B., Tugenberg, T. \& McHorney, C. A. (2003). CONNECT: a measure of continuity of care in mental health services. Mental Health Services Research, 5(4), 209-221.

Weaver, N., Coffey, M. \& Hewitt, J. (2017). Concepts, models and measurement of continuity of care in mental health services: A systematic appraisal of the literature. Journal of Psychiatric and Mental Health Nursing, 24(6), 431-450.

Welsh Government (2005). Raising the standard: the revised adult mental health national service framework and an action plan for Wales. Cardiff: Welsh Assembly Government.

Welsh Government. (2010a). Explanatory memorandum incorporating the regulatory impact assessment, explanatory notes and delegated powers memorandum. Cardiff: Welsh Government.

Welsh Government. (2010b). Mental Health (Wales) Measure 2010. Cardiff: Welsh Government.

Welsh Government. (2010c). Mental Health (Wales) Measure 2010 - Explanatory Notes. Cardiff: Welsh Government.

Welsh Government. (2011a). Implementing the Mental Health (Wales) Measure 2010: Guidance for local health boards and local authorities. Cardiff: Welsh Government.

Welsh Government. (2011b). National Service Model for Local Primary Mental Health Support Services. Cardiff: Welsh Government.

Welsh Government (2012a). Code of practice to Parts 2 and 3 of the Mental Health (Wales) Measure 2010. Cardiff: Welsh Government.

Welsh Government (2012b). Together for Mental Health: a strategy for mental health and wellbeing in Wales. Cardiff: Welsh Government.

Welsh Government. (2013). The duty to review inception report: post-legislative assessment of the Mental Health (Wales) Measure 2010. Cardiff: Welsh Government.

Welsh Government. (2014a). Continuing NHS healthcare The National Framework for Implementation in Wales. Cardiff: Welsh Government. 
Welsh Government. (2014b). Performance data: Mental Health (Wales) Measure 2010. Cardiff: Welsh Government.

Welsh Government. (2014c). The duty to review interim report: post-legislative assessment of the Mental Health (Wales) Measure 2010. Cardiff: Welsh Government.

Welsh Government. (2015). The duty to review final report: post-legislative assessment of the Mental Health (Wales) Measure 2010. Cardiff: Welsh Government.

Welsh Government. (2016). Research to support the duty to review the implementation of the Mental Health (Wales) Measure 2010 - Part 2: Coordination of and Care and Treatment Planning for secondary mental health users. Cardiff: Welsh Government.

Wernick, A. (1991). Promotional Culture. London: SAGE.

Whitaker, E. M. and Atkinson, P. (2019). Authenticity and the interview: a positive response to a radical critique. Qualitative Research, 1-16. doi: http://dx.doi.org/10.1177/1468794118816885

WHO: World Health Organisation. (1948). Official Records of the World Health Organisation, no.2. Geneva: World Health Organisation.

WHO: World Health Organisation. (1999). The world health report: making a difference. Geneva: World Health Organisation.

WHO: World Health Organization. (2001a). Declaration of Helsinki. Bulletin of the World Health Organisation. Helsinki: World Health Organisation.

WHO: World Health Organisation. (2001b). World health report 2001: mental health, new understanding, new hope. Geneva: World Health Organisation. Available at https://www.who.int/whr/2001/media_centre/press_release/en/

WHO. (2008). Integrating mental health into primary care: a global perspective. Helsinki: World Health Organization.

WHO: World Health Organisation. (2012). Depression: a global crisis. Geneva: World Health Organization. Available at https://www.who.int/mental_health/management/depression/wfmh paper_depression_wmhd 2 012.pdf

WHO: World Health Organisation. (2014). Social determinants of mental health. Helsinki: World Health Organisation. Available at https://www.who.int/iris/bitstream/10665/112828/1/9789241506809 eng.pdf?ua=1

WHO: World Health Organization. (2017). ICD-11 Beta Draft. Mental, behavioural or neurodevelopmental disorders. Helsinki: World Health Organisation. Available at https://icd.who.int/en/

Widdicombe, S. (2008). 'Identity as an analysts' and a participants' resourse' in C. Antaki and S. Widdicombe (eds.), Identities in talk (pp.191-206). London: SAGE. 
Wiener, N. (1948). Cybernetics: or control and communication in the animal and the machine. Cambridge, Massachusetts: MIT Press.

Wiering, B., de Boer, D. \& Delnoij, D. (2017). Patient involvement in the development of patientreported outcome measures: a scoping review. Health Expectations, 20(1), 11-23.

Wiggins, S. (2017). Discursive psychology: theory, method and applications. London: SAGE.

Wilkinson, E. (2015). UK NHS staff: stressed, exhausted, burnt out. Lancet, 385(9971), 841-843.

Willig, C. (1999). 'Beyond appearances: a critical realist approach to social constructionist work' in D. Nightingale and J. Cromby (eds.), Social constructionist psychology: a critical analysis of theory and practice (pp.37-51). Buckingham: Open University Press.

Willig, C. (2013). Introducing qualitative research in psychology. Maidenhead: McGraw-Hill.

Wilson, T., Holt, T. \& Greenhalgh, T. (2001). Complexity science: complexity and clinical care. British Medical Journal, 323, 685-688.

Winch, P. (1990). The idea of a social science : and its relation to philosophy (2nd ed.). London: Routledge.

Wood, L. A. and Kroger, R. O. (2000). Doing discourse analysis: Methods for studying action in talk and text. London: SAGE.

Woods, A., Hart, A. \& Spandler, H. (2019). The recovery narrative: politics and possibilities of a genre. Culture, Medicine, and Psychiatry, 1-27.

Wright, M. J., Harmon, K. D., Bowman, J. A., Lewin, T. J. \& Carr, V. J. (2005). Caring for depressed patients in rural communities: General Practitioners' attitudes, needs and relationships with mental health services. Australian Journal of Rural Health 13(1), 21-27.

Wriston, W. B. (1992). The twilight of sovereignty. New York: Macmillan Press. 


\section{Appendices}

\section{Appendix 1: Interview schedule - Phase 1 - Service users}

Introduce yourself and explain the nature of the study:

"Hi. My name is Nick. Thank you for meeting with me today.

You kindly agreed to take part in this research study and I am here today to ask you a few questions about your experiences of care and treatment through the GP practice. The interview should take approximately 45 minutes. There are no right or wrong answers. I just want to know what it's like getting mental healthcare from your GP practice, and how this has affected you. Feel free to talk as much as you want about whatever you think is important about the way your care is delivered under the GP, and how this has impacted your health and wellbeing. Please be aware that I have a duty of care to you as a nurse, so if you should become upset, I may have to stop the interview, at least for a break. If I am concerned about your safety or the safety of someone you know, I may have to let a WWAMH member of staff know."

Check with the person that they are still okay to give their informed consent to be interviewed. Remind them that their name will not be used and that they will not be identified in any way. Remind them that they may stop at any time.

Check digital recorder and microphone are working and sound levels are adequate.

"Before we start, do you have any questions you would like to ask?"

"I am just going to read out the code number for you in this study so that your name can be left out of it and the interview will remain anonymous."

Read our Participant Number Code and Date.

*Please note that prompts below are an aid memoir to help the interviewer in guiding the interview. The prompts are not necessarily questions which will be specifically asked.*

Tell me about what your care was like when you were under the care of the Community Mental Health Team.

Prompts/Probes: Were you ever in hospital? If so, tell me about what your care was like when you were in hospital? What did you think about your care then? Tell me about your care through your Care Coordinator. Did you feel you were well supported by your Care Coordinator? What kind of support was most helpful? What was most unhelpful? When you were discharged from CMHT, how were you told about changes in your care? Tell me about how well this was organised. Is it easy to change aspects of your care?

\section{What kind of support do you now get from your GP practice instead?}

Prompts/Probes: What were your initial expectations when your care was transferred to the GP practice? What has your care been like through your GP practice? What did you find helpful about your care? Less helpful? Have you ever had to change GPs or GP practices? Was it easy to do so? How often do you use services at your GP 
practice? Is this often enough? Can you tell me about an experience where you have had difficulty getting an appointment? Have you ever felt you have had to travel farther than you would like for an appointment? Tell me about this experience. Have you ever felt like you have been 'passed from pillar to post'? What happened then? Have you ever had an experience where you have had to keep repeating your history when meeting a new professional? What happened then?

\section{How easy is it for you to get extra support through your GP?}

Prompts/Probes: Support such as brief CBT, other therapy services like Emotional Coping Skills, information about day centres, drug support agencies etc. Can you tell me about this? Tell me about a time when it was difficult to get extra help. Do you feel your GP practice works well with other services? In what ways? Have you ever had difficulty accessing a service you need? Tell me about this experience. Are you given options other than receiving treatment through medication being prescribed? Like what? If not, what would you like to be offered? What would happen if you needed a referral back to mental health services? Would you know what to do? Did mental health services tell you how you can access them again if needed? Have there been times when you needed help urgently since you were transferred back to the GP practice? Can you tell me about what happened?

There is a lot of talk about Recovery in mental health care nowadays. What does the term Recovery mean to you?

\{Here is a definition that is commonly used: Recovery is a way of living a satisfying, hopeful and contributing life even with the limitations caused by illness. It involves finding new meaning and purpose in life as one grows beyond the effects of mental illness.\}

Prompts/Probes: Do you feel that you are being helped to achieve and maintain your Recovery through your GP practice? Do you feel that you are being helped to develop a personal Recovery plan? Do you feel you are in charge of your treatment and care? Do you feel you are as independent as you would like to be? Do you ever think you are treated differently by people because of your mental illness? How does your GP practice help with this? Are family and friends supportive?

\section{How would you describe your quality of life?}

Prompts/Probes: Tell me about your typical day. What sort of leisure activities do you do? Do you feel that you are occupied each day? Over-occupied? How often do you encounter stress? Are you happy about the level of anxiety there is in your life? Do you do any paid or voluntary work? Tell me about these activities. Tell me about the best day you have had recently. Tell me about your current health. Your physical health. Your mental health. How do you get help when you have problems with your health? Tell me about this.

Having reflected on your experiences of care, is there something else you would like to add that we have not covered?

\section{Is there anything you would like to ask me?}




\section{Appendix 2: Interview schedule - Phase 2 - Service professionals}

What do people tell you about their experiences of care from their GP since being discharged from secondary care?

Prompts / Probes: Tell me about members who have had a positive or negative experience of care under the GP. How does this compare to the care they had with the CMHT or hospital? In what ways does pressure on services limit the availability of good care through the GP?

According to members, how understanding or prepared to listen to mental health concerns are GPs? What kind of confidence do members have in the level of expertise which GPs have for mental health issues as opposed to physical health issues? Do you get the impression that the GP provides a kind of talking therapy or medication treatment? Why is this the case?

What kind of sustained, continuous care do you see people getting through the GP surgery for their mental health?

Prompts / Probes: In what way does this care succeed or fail to meet people's needs, in your opinion? Do people complain about having too frequent a change of GP, or gaps in care? Do people complain about having to repeat their service history to different professionals in primary care?

How well organised is the care for some of your members who have complex needs? In what ways do members describe care through the GP as being flexible and responsive to their needs? How happy are members with the information they receive from the GP, about side-effects of medication, for instance? Other kinds of information? How much freedom do members have in avoiding services they don't want? What ways can they achieve this? How helpful do you think the Care and Treatment Plan is for promoting members' health and recovery? How helpful a tool do you think it is in the context of primary care and treatment? Can you elaborate on that?

\section{How easy or difficult is it for the GP to access secondary care on behalf of members under their care?}

Prompts / Probes: How easy is it for your members to get the treatment they need from different services and professionals over a period of time? Do you feel that there are people who have fallen into a 'gap' between services? Can you describe this?

Part 3 of the Mental Health (Wales) Measure 2010 allows people who have been discharged from secondary care to refer themselves back again within three years. In what ways is it easy or difficult for members to access secondary care in this way? What kinds of difficulties might they face?

In an emergency or crisis situation, how easy or difficult is it for someone to quickly access specialist care? Why is this? Have any of your members deteriorated because they were on a waiting list for a long time? How did this impact their health and recovery? 


\section{What kind of support do your members need in going to the GP if they become unwell?}

Prompts / Probes: How easy is it for people to make and get to an appointment with their GP concerning their mental health? How easy is it for the service user to take the initiative in contacting the GP in order to access care? How much of a role do you find members' families and/or friends have with their ongoing care and treatment? How necessary is this involvement for persons to maintain contact with services? What kind of differences do you see in the healthcare and recovery for people who do have family support compared to those who don't?

How much do you feel the third sector is filling in the gap created by a lack of care provision from primary and secondary services?

Prompts / Probes: In what ways? How important a role do you think the third sector / your organisation has in providing mental health support in Wales? In what ways? Are third sector services over-stretched? Are there ways in which this limits the support you would like to provide?

In what ways does primary care work in partnership with the third sector? In what ways does it not?

\section{Are there ways in which care provided under the GP benefits members' recovery and quality of life?}

\{What is your understanding of the recovery model? Here is a definition that is commonly used: Recovery is a way of living a satisfying, hopeful and contributing life even with the limitations caused by illness. It involves finding new meaning and purpose in life as one grows beyond the effects of mental illness.\}

Prompts / Probes: How does the care members receive from the GP aid their recovery? In what ways are people placed in charge of their health and care under the GP? What kind of choice do members have regarding which GP they see?

How does care under the GP help people to be more hopeful and increase levels of self-esteem?

How does the GP encourage members to undertake vocational or recreational activities?

How does GP support encourage members to connect with and build relations with other people?

What kinds of issues do members have with anxiety, depression or other mental health issues. Can you tell me more about this?

What kind of quality of life do members have who are solely under the care of the GP. How does this compare to members who are under the CMHT? What are the important features of quality of life for members?

\section{After reflecting on these issues, is there something else you would like to add?}

\section{Is there anything you would like to ask me?}




\section{Appendix 3: Participant information sheet}

Study title: Experiences of care continuity and recovery for people at the interface of primary and secondary mental health care in Wales

Principal Investigator: Nick Weaver (RMN)

Project Supervisors: $\quad$ Dr. Michael Coffey; Dr. Jeanette Hewitt; Prof. Deb Fitzsimmons.

$\underline{\text { Introduction }}$

I would like to invite you to take part in my $\mathrm{PhD}$ research study. Before you decide whether or not to participate, I would like you to understand why the research is being done and what it would involve for you. Talk to others if you wish including WWAMH staff members who have been informed about the study.

What is the purpose of the research?

This is a study about what your experiences have been of mental health care if you have had your care transferred from the Community Mental Health Team or psychiatric hospital to your GP practice in the past three years. In particular, I would like to know about how continuous your care has been, and what effect this has had on your quality of life and your personal health.

If you decide to take part, I would like to interview you about your care and treatment, and how this has affected your health and quality of life. The interviews will be carried out in a loose structure so that you will be given the freedom to express and describe exactly what you think and what you have experienced. The thinking behind this study is very much about giving people who are receiving care an opportunity to express their views in an open way, in a safe environment. You will be given the freedom to talk about whatever you want to in relation to your health and care. The information you share will be kept confidential.

Who is organising and funding this study?

This study is a Swansea University research project funded by the RCBC (Research Capacity Building Collaboration - an organisation which supports nurse and health care research) and Health and Care Research Wales. The main research will be done by the principal investigator, Nick Weaver, under the supervision of Dr. Michael Coffey, Dr. Jeanette Hewitt and Prof. Deb Fitzsimmons.

Why have I been invited?

I am inviting people who are receiving treatment through their GP practice and who have also been discharged from a Community Mental Health Team or psychiatric hospital within the last three years to participate in the study. This will allow us to find out more about your experiences of care and how this has impacted your health and quality of life.

Do I have to take part?

Participation in the study is entirely voluntary. If you do decide to take part then you are free to withdraw at any time, without giving a reason. This will not affect the care you receive. 
What will I have to do if I take part?

You will be invited to be interviewed by the principal investigator about your views of the treatment you have been receiving through your GP practice. You will also be interviewed about the impact this has had on your health, wellbeing and quality of life. It is estimated this interview will take about 45 minutes to complete.

Where will the interviews be held?

The interviews may be held in a meeting room at the WWAMH Carmarthen Offices (The Mount, 18 Queen Street, Carmarthen SA31 1JT). A WWAMH staff member will be available on premises as well as the principal investigator if you have any further questions or if you should need further support. Tea and coffee facilities will be provided. Alternatively, if you find it more convenient, or you have mobility problems, the principal researcher is happy to come to your home to interview you.

Are there any risks or inconveniences?

I do not think there are any risks involved in this study. You may find the interview deals with sensitive issues or experiences, if you wish to raise them in the course of the interview. You are reminded that your participation is entirely optional and you are free to withdraw from the study at any time. There will be a WWAMH staff member available on premises should you need any support because of any sensitive issues discussed during the interview.

What benefits are there for me?

I cannot promise the study will help you but the information I get from this study may help improve the support of people with mental illness.

Who has reviewed this research?

All research carried out through Swansea University is looked at by a group of people, called the College of Health and Human Sciences Research Ethics Committee, to protect your interests. This study has been reviewed and given favourable opinion by this committee.

\section{What if there is a problem?}

If you have concerns about any aspect of this study, or would like further information, you should ask to speak to the principal investigator, Nick Weaver, who will seek to answer your questions. You can contact this person by telephone on 07502523288 or by emailing him at656890@swansea.ac.uk.

If you remain unhappy and wish to complain formally, you can do this through the University complaints procedure. This procedure can be initiated by contacting Dr Michael Coffey (email: m.j.coffey@swansea.ac.uk).

How will you keep information about me confidential?

The information you give us and that I collect about you through the interviews will be treated in confidence. I will do this in several ways:

1. Any personal information that can identify you will only be seen by the principal investigator. 
2. All information collected about you during the course of the research will be kept strictly confidential. I will remove any personal information from documents that could potentially identify you.

3. Any personal information will be stored safely and securely on password-protected computers or in locked cabinets in secure University premises. These will only be accessible by the principal investigator.

4. Digital recordings of interviews will not include your name because a code number will be used.

5. At no time will you be identified in reports, publications or presentations. Names and other identifiable information will be replaced with codenames to ensure that all data remains confidential.

6. Data collected for this research study will be stored securely for up to 5 years and then destroyed.

Finally, anything you tell us will be treated in confidence unless I am concerned about your safety or the safety of someone else, or if I become aware of professional malpractice. In this case I would have to discuss my concerns with you and support staff in WWAMH.

What will happen if I don't want to continue with the study?

You may withdraw from the study at any time.

Will WWAMH staff know about the research?

I will only report what has been discussed in interview if I am concerned for your safety or the safety of others. I will only discuss a concern with an appropriate WWAMH staff member. Your GP will not be informed about your involvement in this study.

\section{What will happen to the results of the study?}

The results of the study will be published as research papers in professional journals. The principal investigator may also write articles in healthcare magazines and service user publications and present results at conferences. Information may be placed on the WWAMH website or linked to by this website. Results will also be made available to the Welsh Government and policy makers. The results and details of this study will also be published in a $\mathrm{PhD}$ thesis authored by the principal investigator. You will not be identified in any publication, report or presentation.

\section{What do I do now?}

If you have any question or concerns, please contact Nick Weaver (contact details are on the back page of this booklet). You can keep this information booklet for future reference. If you are undecided, you may wish to discuss whether to take part in the study with a WWAMH staff member, a family member or friend.

If you have fully understood the information and would like to take part in the study, please contact the principal investigator, Nick Weaver, either by email (656890@swansea.ac.uk) or by telephone (07502 523 288). You can then arrange a convenient time and place for the interview. Please also sign and date the consent form which is provided with this participant information sheet. When you attend the interview, bring the completed consent form with you and give it to the principal investigator. 
Do not give the consent form to a WWAMH staff member as this will breach your anonymity.

To be eligible to participate, you must have been discharged within the last three years from a Community Mental Health Team or psychiatric hospital where you were treated for a mental health issue. You must also be between 18 and 65 years of age.

Please note that I can only interview 20 people at the most. For this reason I will only interview the first 20 people who come forward. If you want to be interviewed but 20 people have already agreed to be interviewed, you will be placed on a reserve list.

You have a minimum of 14 days to think about whether you want to be involved in this study.

Once the principal investigator has received your completed consent form, he will arrange for you to participate in an interview with you at a suitable time and in a suitable place, either at the WWAMH Carmarthen Offices or in your home.

If you do not wish to take part in the study, you do not need to do anything else.

Thank you very much for your time and interest.

Nick Weaver (RMN) - Principal Investigator

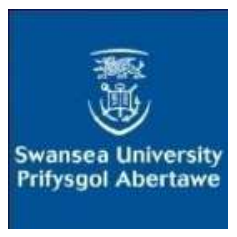

For more information about this research project, or to take part in the study, please speak to a WWAMH member of staff or contact:

Nick Weaver

Principal Investigator, Swansea University

Tel: 07502523288

Email:656890@swansea.ac.uk

If you remain unhappy and wish to complain formally, you can do this through the University complaints procedure (contact Dr. Michael Coffey; email: m.j.coffey@swansea.ac.uk). 


\section{Appendix 4: Consent form}

\section{Study Title: Experiences of care continuity and recovery for people at the interface}

\section{of primary and secondary mental health care in Wales}

Please Initial Box

1. I confirm that I have read and understood the participant information sheet for the above study and have had the opportunity

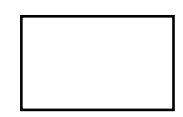
to ask questions.

2. I understand that my participation is voluntary and that I am free to withdraw at any time, without giving any reason, without my

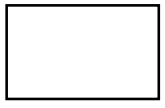
support and treatment through West Wales Action for Mental Health or my legal rights being affected.

3. I understand that my participation in an interview will be recorded on digital audio recording device and give permission for this data

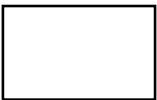
to be securely stored and used to inform the research process of the above study.

4. I agree to take part in the above study.

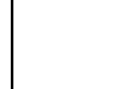

Name of Patient:

Date:

Signature:

Investigator:

Date:

Signature: 


\section{Appendix 5: Recruitment Poster}

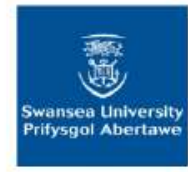

\section{"robcuales}

sing

(O)

\section{Research Participants Needed}

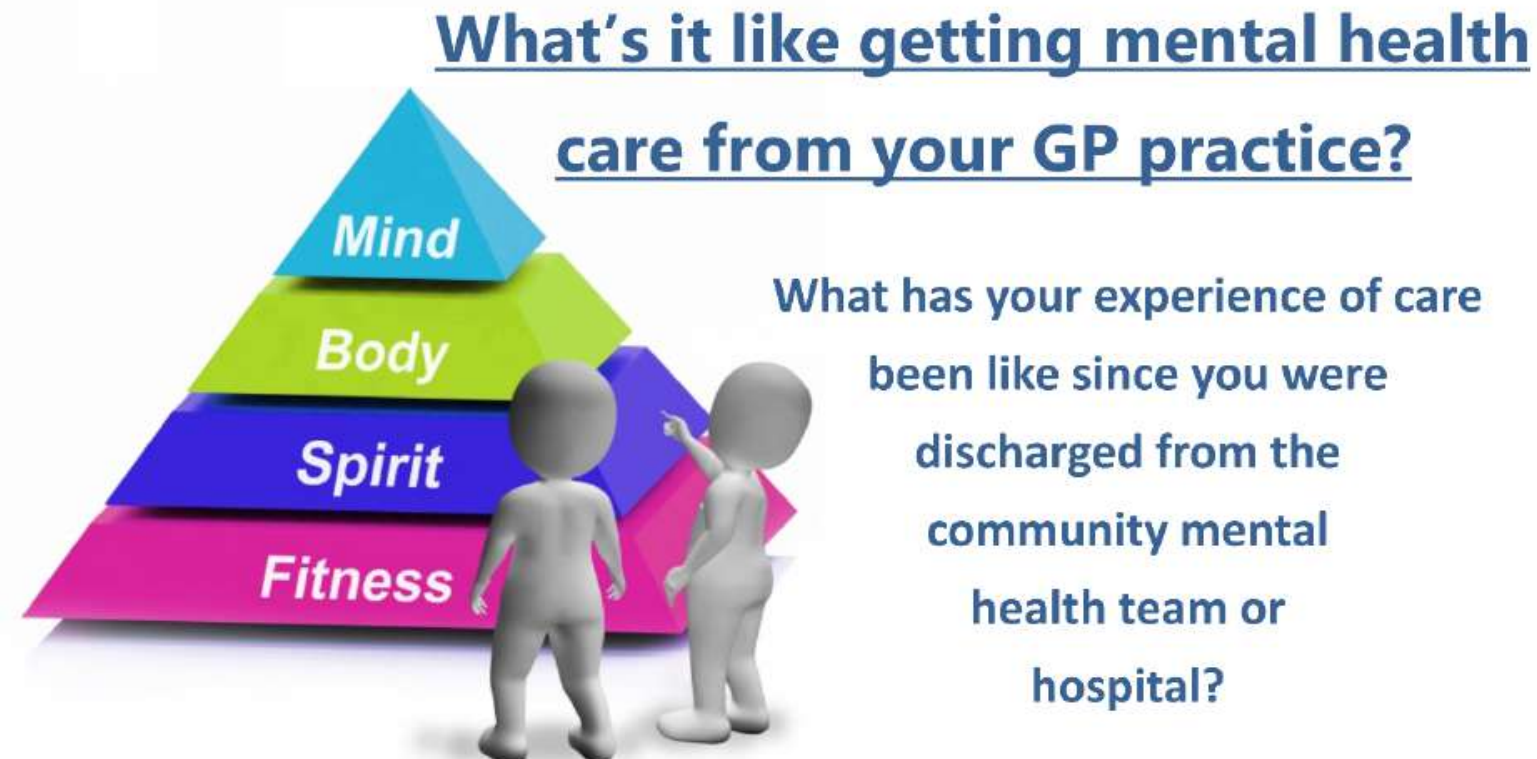

\section{Volunteers are needed to take part in some interviews in which you can be frank and honest}

Who? You must be between 18 and 65 . You must have been under the care of the GP only for at least six months in the last three years but also have spent some time under the community mental health team or hospital where you were treated for your mental health.

Where? Interviews will be held in WWAMH Carmarthen Offices or on associated charity premises. Alternatively, you can be interviewed in your home if this is more convenient for you.

How long will it take? 45 minutes approximately.

How will my data be used? Your data will be anonymised. Information gained will help create a picture of how well care is being provided through GP practices.

\section{Interested?}

Ask a staff member for a leaflet or a participant information booklet.

Or contact the main researcher, Nick Weaver:

email: 656890@swansea.ac.uk tel: $\quad 07502523288$

Please note: A maximum of 20 people will be interviewed. If you want to be interviewed but 20 people have already agreed to be interviewed, you will be placed on a reserve list. 


\section{Appendix 6: Recruitment leaflet}

Since the leaflet is in brochure format, it is not possible to place an exact copy of it in this thesis. However, all text used in the leaflet is provided below.

\section{$\underline{\text { Research participants needed }}$}

\section{What's it like getting mental health care from your GP practice?}

There is an opportunity for you to take part in a research study that looks at mental health care from the GP practice. If you have been discharged within the last three years from a Community Mental Health Team or psychiatric hospital where you were treated for a mental health issue, you can help with this research study.

This research project is about finding out what your experiences have been like whilst your care has changed over to the GP practice. What has your experience been like been like since you were discharged from the Community Mental Health Team or hospital? What has been the impact on your health and quality of life? Have you been supported in developing a healthy recovery?

If you decide to take part, you will have the opportunity to take part in an interview. During these interviews you will be asked about your care and treatment, and how this has affected your health and quality of life. You will have an opportunity to be frank and honest during these interviews. The idea is to create a safe environment in which you can express your views freely.

$\underline{\text { If you are interested }}$

Please ask a WWAMH staff member for an information booklet which will provide you with further details.

You can also contact Nick Weaver, who will be conducting the research:

Email:656890@swansea.ac.uk

Tel.: 07502523288

Who? - You must be between 18 and 65. You must have been discharged within the last three years from the Community Mental Health Team or psychiatric hospital where you were treated for a mental health issue.

Where? - Interviews will be held in WWAMH Carmarthen Offices (The Mount, 18 Queen Street, Carmarthen SA31 1JT). Alternatively, you can be interviewed in your home if this is more convenient for you.

How long will it take?- 45 minutes approximately.

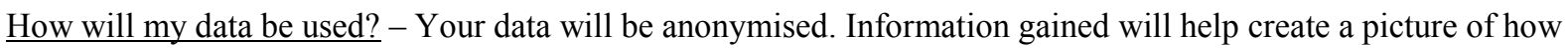
well care is being provided through GP practices.

Please note: Interviews will be for the first 20 people who come forward. Anyone interested after this will be placed on a reserve list. 


\section{Appendix 7: Participant characteristics}

Table 4 - Phase 1 participant characteristics

\begin{tabular}{|c|c|c|c|c|c|}
\hline Pseudonym & $\begin{array}{l}\text { Code } \\
\text { number }\end{array}$ & Age-range & Gender & $\begin{array}{l}\text { Involvement } \\
\text { with third sector } \\
\text { day centre? }\end{array}$ & $\underline{\text { Reported SMI }}$ \\
\hline Alun & 01 & $50-60$ & $\mathrm{M}$ & Yes & Bipolar \\
\hline Patricia & 02 & $40-50$ & $F$ & Yes & Depression \\
\hline Bernadette & 03 & $40-50$ & $\mathrm{~F}$ & Yes & Bipolar \\
\hline Katy & 04 & $30-40$ & $\mathrm{~F}$ & Yes & $\begin{array}{l}\text { Depression, } \\
\text { anxiety, anorexia }\end{array}$ \\
\hline Doris & 05 & $40-50$ & $\mathrm{~F}$ & Yes & $\begin{array}{l}\text { Depression and } \\
\text { anxiety }\end{array}$ \\
\hline Tricia & 06 & $30-40$ & $F$ & Yes & Anxiety \\
\hline Susan & 07 & $40-50$ & $\mathrm{~F}$ & Yes & $\begin{array}{l}\text { Depression and } \\
\text { anxiety }\end{array}$ \\
\hline James & 08 & $50-60$ & $\mathrm{M}$ & Yes & Anxiety \\
\hline Matthew & 09 & $30-40$ & $\mathrm{M}$ & Yes & $\begin{array}{l}\text { Epilepsy and } \\
\text { related psychosis }\end{array}$ \\
\hline Mavis & 10 & $50-60$ & $F$ & Yes & Depression \\
\hline Thomas & 11 & $50-60$ & $\mathrm{M}$ & Yes & $\begin{array}{l}\text { Depression and } \\
\text { anxiety }\end{array}$ \\
\hline Angela & 12 & $40-50$ & $\mathrm{~F}$ & Yes & Bipolar \\
\hline Marina & 13 & $40-50$ & F & Yes & PTSD \\
\hline Alexandra & 14 & $40-50$ & $\mathrm{~F}$ & Yes & $\begin{array}{l}\text { Bipolar and } \\
\text { anxiety }\end{array}$ \\
\hline Sandra & 15 & $40-50$ & $\mathrm{~F}$ & Yes & Bipolar \\
\hline Ian & 16 & $30-40$ & $\mathrm{M}$ & None reported & PTSD \\
\hline
\end{tabular}


Table 5 - Phase 2 participant characteristics

\begin{tabular}{|c|c|c|c|c|c|}
\hline Pseudonym & $\begin{array}{l}\text { Code } \\
\text { number }\end{array}$ & Age-range & $\underline{\text { Gender }}$ & $\begin{array}{l}\text { Organisation } \\
\text { type }\end{array}$ & Profession \\
\hline Beth & 01 & $50-60$ & $\mathrm{~F}$ & $\begin{array}{l}\text { Third sector day } \\
\text { centre }\end{array}$ & $\begin{array}{l}\text { Third sector } \\
\text { manager }\end{array}$ \\
\hline Anna & 02 & $40-50$ & $\mathrm{~F}$ & $\begin{array}{l}\text { Third sector day } \\
\text { centre }\end{array}$ & $\begin{array}{l}\text { Senior third sector } \\
\text { support worker }\end{array}$ \\
\hline Roberta & 03 & $30-40$ & $\mathrm{~F}$ & $\begin{array}{l}\text { Third sector } \\
\text { outreach }\end{array}$ & $\begin{array}{l}\text { Third sector } \\
\text { manager }\end{array}$ \\
\hline Dylan & 04 & $50-60$ & $\mathrm{M}$ & Freelance & $\begin{array}{l}\text { Senior third sector } \\
\text { support worker }\end{array}$ \\
\hline Maria & 05 & $50-60$ & $\mathrm{~F}$ & $\begin{array}{l}\text { Third sector day } \\
\text { centre }\end{array}$ & $\begin{array}{l}\text { Third sector } \\
\text { manager }\end{array}$ \\
\hline Kaitlin & 06 & $20-30$ & $\mathrm{~F}$ & $\begin{array}{l}\text { Third sector day } \\
\text { centre }\end{array}$ & $\begin{array}{l}\text { Junior third sector } \\
\text { support worker }\end{array}$ \\
\hline Tony & 07 & $30-40$ & $\mathrm{M}$ & $\begin{array}{l}\text { Third sector day } \\
\text { centre }\end{array}$ & $\begin{array}{l}\text { Third sector } \\
\text { manager }\end{array}$ \\
\hline Tom & 08 & $30-40$ & $\mathrm{M}$ & $\begin{array}{l}\text { Third sector day } \\
\text { centre }\end{array}$ & $\begin{array}{l}\text { Senior third sector } \\
\text { support worker }\end{array}$ \\
\hline Hannah & 09 & $30-40$ & $\mathrm{~F}$ & $\begin{array}{l}\text { Third sector day } \\
\text { centre }\end{array}$ & $\begin{array}{l}\text { Junior third sector } \\
\text { support worker }\end{array}$ \\
\hline Luke & 10 & $30-40$ & $\mathrm{M}$ & $\begin{array}{l}\text { Third sector day } \\
\text { centre }\end{array}$ & $\begin{array}{l}\text { Third sector } \\
\text { manager }\end{array}$ \\
\hline Sheryl & 11 & $50-60$ & $\mathrm{~F}$ & $\begin{array}{l}\text { Third sector day } \\
\text { centre }\end{array}$ & $\begin{array}{l}\text { Third sector area } \\
\text { manager }\end{array}$ \\
\hline Lee & 12 & $20-30$ & $\mathrm{M}$ & $\begin{array}{l}\text { Third sector day } \\
\text { centre }\end{array}$ & $\begin{array}{l}\text { Junior third sector } \\
\text { support worker }\end{array}$ \\
\hline Robert & 13 & $30-40$ & $\mathrm{M}$ & General Practice & $\begin{array}{l}\text { General } \\
\text { Practitioner }\end{array}$ \\
\hline Tamsen & 14 & $40-50$ & $\mathrm{~F}$ & General Practice & $\begin{array}{l}\text { General } \\
\text { Practitioner }\end{array}$ \\
\hline Alex & 15 & $30-40$ & $\mathrm{M}$ & General Practice & $\begin{array}{l}\text { General } \\
\text { Practitioner }\end{array}$ \\
\hline Olivia & 16 & $40-50$ & $\mathrm{~F}$ & $\begin{array}{l}\text { Third sector day } \\
\text { centre }\end{array}$ & $\begin{array}{l}\text { Third sector } \\
\text { manager }\end{array}$ \\
\hline
\end{tabular}




\section{Appendix 8: Transcribing conventions and symbols}

\begin{tabular}{|c|c|c|}
\hline[ & $\begin{array}{l}\text { Researcher: quite a [ while } \\
\text { Participant: [ yeah.... }\end{array}$ & $\begin{array}{l}\text { Left brackets indicate the point at which a current } \\
\text { speaker's talk is overlapped by another's talk. }\end{array}$ \\
\hline$==$ & $\begin{array}{l}\underline{\mathrm{R}}: \text { that I'm aware of }= \\
\underline{\mathrm{P}}:=\text { Yes. Would you } \\
\text { confirm that? }\end{array}$ & $\begin{array}{l}\text { Equal signs, one at the end of a line and one at } \\
\text { the beginning, indicate no gap between the two } \\
\text { lines. }\end{array}$ \\
\hline$(.4)$ & Yes (.2) yeah & $\begin{array}{l}\text { Numbers in parentheses indicate elapsed time in } \\
\text { silence in tenths of a second. }\end{array}$ \\
\hline (.) & To get (.) treatment & $\begin{array}{l}\text { A dot in parentheses indicates a tiny gap, } \\
\text { probably no more than one-tenth of a second. }\end{array}$ \\
\hline ( ) & $\begin{array}{l}\text { Future risks and ( ) and life } \\
\text { ( ) }\end{array}$ & $\begin{array}{l}\text { Empty parentheses indicate the transcriber's } \\
\text { inability to hear what was said. }\end{array}$ \\
\hline (word) & $\begin{array}{l}\text { Would you see (there) } \\
\text { anything positive? }\end{array}$ & Parenthesised words are possible hearings. \\
\hline$(())$ & Confirm that ((continues)) & $\begin{array}{l}\text { Double parentheses contain author's descriptions } \\
\text { rather than transcriptions. }\end{array}$ \\
\hline$<<$ word $>>$ & $<<$ CMHT $>>$ & $\begin{array}{l}\text { Place and people names are omitted and } \\
\text { substituted with generic labels to preserve } \\
\text { anonymity e.g. }<<\mathrm{CMHT}>>\text {. }\end{array}$ \\
\hline
\end{tabular}

(see Silverman, 2014, p.449) 


\section{Appendix 9: Inductive search terms and Boolean search strings}

\section{MeSH terms and related search string}

As well as using key terms and Boolean search strings in the various subject areas, I made use of MeSH (Medical Subject Headings) terms to enhance and focus my searches to be within the field of mental healthcare and services (Richter and Austin, 2012). These were combined with Boolean search strings for the various subject domains reviewed in Chapter two. I developed a set of MeSH terms applicable to the ProQuest portal which can be found at https://search-proquest-com.openathensproxy.swan.ac.uk/) I used these MeSH terms to delimit my inductive searches to the field of mental healthcare and mental health services. After thorough examination of the 'MeSH 2018 Thesaurus' and tree-structure of subject headings within the ProQuest bibliographic database search engine, the following set of terms were employed:

"Mental health care"

"Mental disorders"

"Mental health services" NOT "Counseling"

"Mental health"

"Community psychiatry"

"Forensic psychiatry"

Note that for the MeSH term "Mental health services" I excluded the sub-tree under this heading for "Counseling" using the Boolean operator 'NOT', which after examination I did not consider to be a subject area directly relevant to my study. I also used a command-line approach for entering Boolean string searches into the ProQuest site which was the most accurate and error-free way for me to perform my inductive searches. The resultant Boolean string for MeSH terms, which I used in combination with key term strings for each of the subject areas, was as follows:

mainsubject.EXACT("Mental health care") OR mesh.EXACT.EXPLODE("Mental disorders") OR (mesh.EXACT.EXPLODE("Mental health services") NOT mesh.EXACT.EXPLODE("Counseling")) OR mesh.EXACT.EXPLODE("Mental health") OR mesh.EXACT.EXPLODE("Community psychiatry") OR mesh.EXACT.EXPLODE("Forensic psychiatry")

Note the use of the 'EXPLODE' term which was necessary for my searches to automatically explore the tree-structure of subject headings subordinate to the MeSH terms' positions in the entire database. 


\section{Search terms and search strings for care continuity}

The following search terms and Boolean search string were used to perform an inductive search of literature within the care continuity subject domain:

\{care continuity; continuity of care; care coordination; case management; care planning\}

From these terms, I generated the following Boolean string which was combined with the MeSH term string above, using the Boolean operator 'AND', to produce the complete search sting applied to bibliographic databases:

TI("care contin*") OR TI("continuity of care") OR TI("care coordin*") OR TI("care co-ordin*") OR TI("case manage*") OR TI("care plan*") OR AB("care contin*") OR AB("continuity of care") OR $\mathrm{AB}($ "care coordin*") OR AB("care co-ordin*") OR AB("case manage*") OR AB("care plan*")

A brief explanation of syntax is necessary at this point. 'TI' stands for title, meaning that strings immediately after it within brackets will be searched for in publication titles. 'AB' stands for abstract, meaning that strings immediately after it within brackets will be searched for in the publication abstract. All key terms for the subject domain were therefore applied within this command line Boolean search string to be searched for within titles and abstracts of potential articles.

\section{Search terms and search strings for recovery}

The following search terms were used for the recovery subject domain. These were generated from initial textbook literature on recovery which I read during the course of conducting this project (Amering and Schmolke, 2009; Pigrim and McCranie, 2013; Repper and Perkins, 2003; Slade, 2009). \{recovery; empowerment; recovery star; WRAP; wellness and recovery action plan; wellness recovery action plan; tidal model; social inclusion; personal journey; critique of services\}

From these terms, I generated the following Boolean string:

TI(recovery) OR TI(empower*) OR TI("recovery star") OR TI(WRAP) OR TI("wellness and recovery action plan") OR TI("wellness recovery action plan") OR TI("tidal model") OR TI("social inclusion") OR TI("personal journey") OR TI("critique of services") OR AB(recovery) OR AB(empower*) OR $\mathrm{AB}$ ("recovery star") OR AB(WRAP) OR AB("wellness and recovery action plan") OR AB("wellness recovery action plan") OR $\mathrm{AB}$ ("tidal model") $\mathrm{OR} \mathrm{AB}$ ("social inclusion") $\mathrm{OR} \mathrm{AB}$ ("personal journey") OR AB("critique of services") 
Because there were so many articles on recovery and because I was interested in issues and difficulties surrounding clarification, versions and appropriations of the concept (Barker, 2003; Mind, 2008; Pilgrim, 2008). I added a further search string to refine my search according to this line of inquiry. The string was based on the following terms:

\{concept; model; theory; colonisation; tokenisation; co-opt\}

The following string was generated out of these terms:

TI(concept*) OR TI(model*) OR TI(theor*) OR TI(colonis*) OR TI(tokenis*) OR TI(co-opt*) OR $\mathrm{AB}$ (concept*) OR AB(model*) OR AB(theor*) OR AB(colonis*) OR AB(tokenis*) OR AB(co-opt*)

It should be noted that for all my searches within the ProQuest database I searched only the following, relevant subsidiary databases: the Education database; Health and medical collection database; Healthcare administration database; Nursing and allied health database; Public health database; Social science database; and the Sociology database. 
Appendix 10: Figure 16: Example of individual participant concept map: Tricia, whose talk is analysed in section 6.3.1

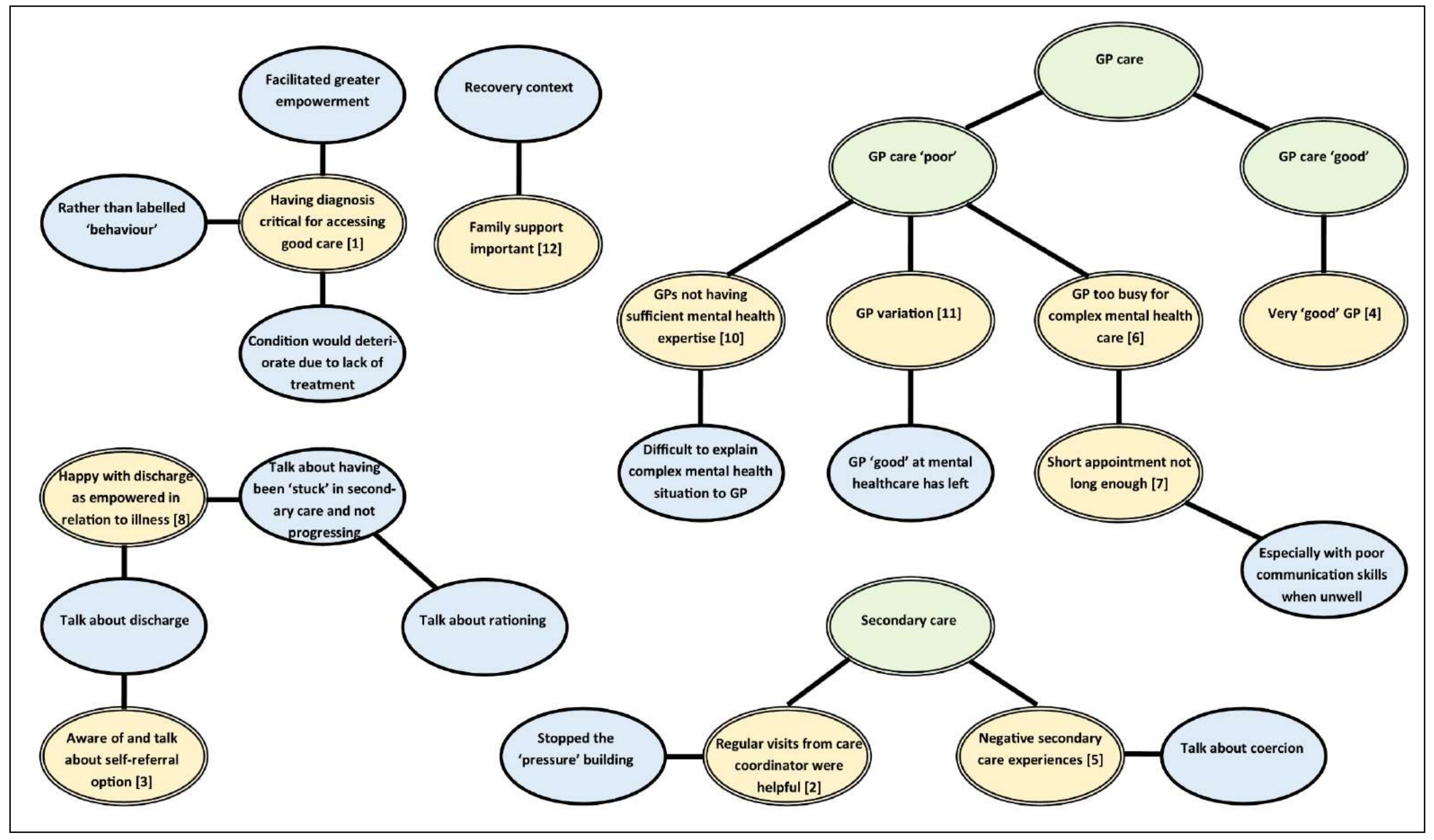


Appendix 11: Table 6: Summary of findings of studies included in care continuity review

\begin{tabular}{|c|c|c|c|c|c|c|c|c|}
\hline Article & $\begin{array}{l}\text { Study } \\
\text { Group } \\
\text { / Scale }\end{array}$ & Target Population & Sample Size & Data Collection & Data Analysis & $\begin{array}{l}\text { Findings / Domains / Components of Care } \\
\text { Continuity }\end{array}$ & Study Limitations & $\begin{array}{l}\text { Quality } \\
\text { Appraisal } \\
\text { Rating (CASP } \\
\text { and COSMIN) }\end{array}$ \\
\hline $\begin{array}{l}\text { Belling et } \\
\text { al. (2011) }\end{array}$ & $\begin{array}{l}\text { ECHO } \\
\text { Group }\end{array}$ & $\begin{array}{l}\text { Random sample of } \\
\text { health and social care } \\
\text { professionals. }\end{array}$ & $n=113$ & $\begin{array}{l}\text { Structured questionnaire } \\
\text { Semi-structured interviews }\end{array}$ & $\begin{array}{l}\text { Data systematically coded, } \\
\text { categorised and analysed } \\
\text { using framework analysis. }\end{array}$ & 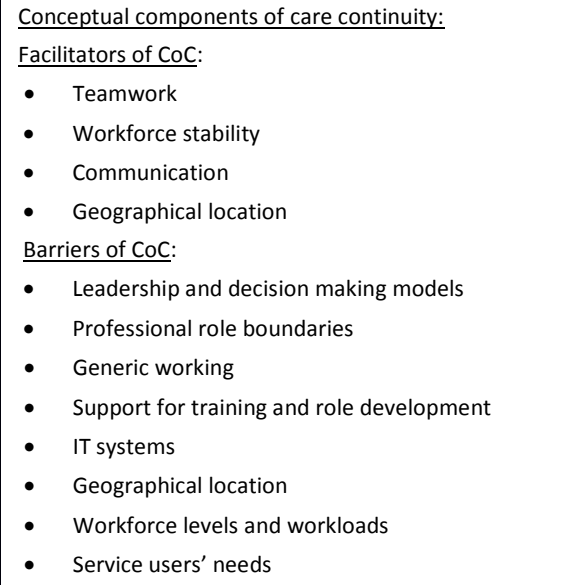 & $\begin{array}{l}\text { Generalisation constrained by limited geographical } \\
\text { location of study. }\end{array}$ & $\begin{array}{l}\text { Weak - } 1 \\
\text { Moderate - } 6 \\
\text { Strong - } 4 \\
\text { Overall - Strong }\end{array}$ \\
\hline $\begin{array}{l}\text { Burns et al. } \\
\text { (2009) }\end{array}$ & $\begin{array}{l}\text { ECHO } \\
\text { Group }\end{array}$ & $\begin{array}{l}\text { People with long-term } \\
\text { psychotic disorders } \\
\text { sampled from the } \\
\text { caseloads of seven } \\
\text { community mental } \\
\text { health teams (CMHTs). }\end{array}$ & $n=180$ & $\begin{array}{l}\text { Case notes, demographic and } \\
\text { illness data and interviews } \\
\text { involving various instruments: } \\
\text { - Camberwell Assessment of } \\
\text { Need (CAN) } \\
\text { - CONTINU-UM } \\
\text { - Scale to Assess Therapeutic } \\
\text { Relationships in } \\
\text { Community Mental Health } \\
\text { Care (STAR) } \\
\text { - Data on contact with } \\
\text { services, number of } \\
\text { professional seen and } \\
\text { information flow from } \\
\text { CMHT records }\end{array}$ & $\begin{array}{l}\text { Factor analysis employed to } \\
\text { operationalise domains of } \\
\text { the Freeman model in } \\
\text { relation to components of } \\
\text { data collection. }\end{array}$ & 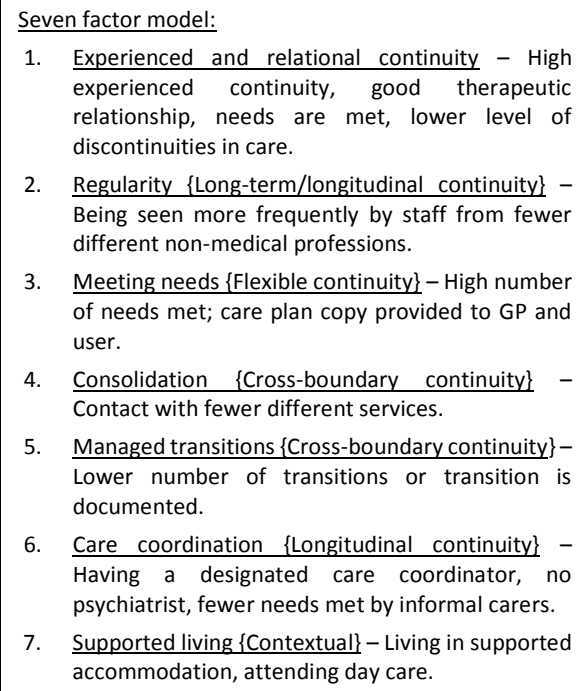 & $\begin{array}{l}\text { - Psychometrically valid confirmation of Freeman } \\
\text { model through factor analysis. } \\
\text { - More than half of eligible service users who met } \\
\text { inclusion criteria declined to participate ( } n=318 \text { ). It } \\
\text { is possible that those who declined may be less } \\
\text { engaged with or favourably disposed to services. } \\
\text { - Weak correlations in factor analysis not removed. } \\
\text { - Data collection limited by quality of case notes and } \\
\text { information provided on file by individual CMHTs. } \\
\text { - Choice of component input for each domain may be } \\
\text { a matter of interpretation. }\end{array}$ & $\begin{array}{l}\text { Weak - } 1 \\
\text { Moderate - } 7 \\
\text { Strong }-5 \\
\text { Overall - Strong }\end{array}$ \\
\hline
\end{tabular}




\section{Appendix 11: Table 6: Summary of findings of studies included in care continuity review}

\begin{tabular}{|c|c|c|c|c|c|c|c|c|}
\hline Article & $\begin{array}{l}\text { Study } \\
\text { Group } \\
\text { / Scale }\end{array}$ & Target Population & Sample Size & Data Collection & Data Analysis & $\begin{array}{l}\text { Findings / Domains / Components of Care } \\
\text { Continuity }\end{array}$ & Study Limitations & $\begin{array}{l}\text { Quality } \\
\text { Appraisal } \\
\text { Rating (CASP } \\
\text { and COSMIN) }\end{array}$ \\
\hline $\begin{array}{l}\text { Catty et al. } \\
\text { (2011) }\end{array}$ & $\begin{array}{l}\text { ECHO } \\
\text { Group }\end{array}$ & $\begin{array}{l}\text { Service users under the } \\
\text { CMHT with psychotic } \\
\text { disorders from seven } \\
\text { CMHTs. }\end{array}$ & $n=180$ & $\begin{array}{l}\text { - Interviews conducted at } \\
\text { baseline and one and two } \\
\text { year follow-up. } \\
\text { - Data collected on patterns } \\
\text { of contact with services, } \\
\text { breaks in care, hospital } \\
\text { stays and psychiatric } \\
\text { symptoms. } \\
\text { - Global Assessment of } \\
\text { Functioning (GAF), } \\
\text { Camberwell Assessment of } \\
\text { Need (CAN), CONTINU-UM, } \\
\text { Manchester Short } \\
\text { Assessment of Quality of } \\
\text { Life (MANSA). }\end{array}$ & $\begin{array}{l}\text { - Analysis 1: Assessment } \\
\text { of associations between } \\
\text { variables as explanatory } \\
\text { variables and continuity } \\
\text { factor scores (univariate } \\
\text { and multifactorial } \\
\text { analysis). } \\
\text { - Analysis 2: Assessment } \\
\text { of associations between } \\
\text { continuity factors and } \\
\text { change in clinical and } \\
\text { social functioning (linear } \\
\text { regression, ANOVA). } \\
\text { - Analysis 3: Exploration } \\
\text { of impact of CoC factors } \\
\text { on clinical and social } \\
\text { outcomes. }\end{array}$ & $\begin{array}{l}\text { Conceptual components of care continuity: } \\
\text { - Having a designated care coordinator valuable for } \\
\text { improving CoC. } \\
\text { - Better quality of life is associated with greater } \\
\text { experienced and relational continuity } \\
\text { - Relationship between CoC factors and clinical, } \\
\text { social and functional outcomes is not uni- } \\
\text { directional but a dynamic process. }\end{array}$ & $\begin{array}{l}\text { - Ethical approval not specified. } \\
\text { - Changes in clinical and social functioning scores not } \\
\text { of a great magnitude limiting the degree to which } \\
\text { causal links may be inferred. } \\
\text { - No differential relations provided between CoC and } \\
\text { narrower diagnostic groups such as bipolar disorder. } \\
\text { - Positive relationship between outcomes and } \\
\text { experienced and relational CoC may have been } \\
\text { inflated by absence of drop-outs. } \\
\text { - Non-participants may have been less favourably } \\
\text { disposed towards services and would have scored } \\
\text { lower for experienced and relational CoC. } \\
\text { - Drop outs likely to be younger. } \\
\text { - Potential response bias. }\end{array}$ & $\begin{array}{l}\text { Weak - } 2 \\
\text { Moderate - } 10 \\
\text { Strong - } 2 \\
\text { Overall - Weak }\end{array}$ \\
\hline $\begin{array}{l}\text { Crawford } \\
\text { et al. } \\
\text { (2004) }\end{array}$ & $\begin{array}{l}\text { ECHO } \\
\text { Group }\end{array}$ & $\begin{array}{l}\text { Research articles and } \\
\text { grey literature from The } \\
\text { Cochrane Library, } \\
\text { Medline, Embase, } \\
\text { PsycINFO, AHMED, } \\
\text { CINAHL, HMIC, HELMIS, } \\
\text { Web of Science and } \\
\text { SIGLE. }\end{array}$ & $\begin{array}{l}\text { Literature } \\
\text { review; } \\
n=60\end{array}$ & Narrative review & Narrative review & $\begin{array}{l}\text { Various factors and themes: } \\
\text { 1. Sustained contact with services } \\
\text { 2. Breaks in service delivery } \\
\text { 3. Seeing the same staff member } \\
\text { 4. Coordination of health and social care } \\
\text { professionals and services } \\
\text { 5. Experienced continuity of care } \\
\text { Some evidence of a loose association between CoC and } \\
\text { outcomes. }\end{array}$ & $\begin{array}{l}\text { - Quality appraisal approach for literature under } \\
\text { review not specified. } \\
\text { - Articles reviewed were a mix of observational, } \\
\text { experimental and qualitative research meaning that } \\
\text { meta-analysis of data is not possible. Narrative } \\
\text { review conducted instead. } \\
\text { - Difficulties encountered identifying papers for } \\
\text { review. } \\
\text { - Limited resources meant that papers not published in } \\
\text { English were not included. }\end{array}$ & $\begin{array}{l}\text { Weak - } 1 \\
\text { Moderate - } 6 \\
\text { Strong - } 0 \\
\text { Overall - Moderate }\end{array}$ \\
\hline $\begin{array}{l}\text { Jones et al. } \\
\text { (2009) }\end{array}$ & $\begin{array}{l}\text { ECHO } \\
\text { Group }\end{array}$ & $\begin{array}{l}\text { Service users diagnosed } \\
\text { with long-term } \\
\text { psychotic disorders and } \\
\text { non-psychotic } \\
\text { disorders and their } \\
\text { carers. }\end{array}$ & $\begin{array}{l}31 \text { service users } \\
(n=31) \\
14 \text { carers } \\
(n=14)\end{array}$ & $\begin{array}{l}\text { Qualitative interviews framed } \\
\text { around service user's illness } \\
\text { career. }\end{array}$ & $\begin{array}{l}\text { Thematic analysis. 'Patient } \\
\text { career' concept used to } \\
\text { frame patient accounts of } \\
\text { experiences of mental } \\
\text { health care system. }\end{array}$ & $\begin{array}{l}\text { Five conceptual themes: } \\
\text { - } \frac{\text { Relational continuity or discontinuity }}{\text { changes of staff. }} \\
\text { - Repeated } \\
\frac{\text { Depersonalised transitions }}{\text { discharge, between teams due to a change of }} \\
\text { residence or team restructuring. } \\
\text { - Invisibility and crisis - Invisibility of user in run up to } \\
\text { crisis point. } \\
\text { Communication gaps - Discontinuities in } \\
\text { communication between services. } \\
\text { Social vulnerability - Complexity of service user's } \\
\text { needs and uncertainties surrounding their illness } \\
\text { and daily lives leads to social vulnerability. }\end{array}$ & $\begin{array}{l}\text { - Limitations to research study methodology not } \\
\text { discussed. } \\
\text { - Limited discussion on impact and further research. }\end{array}$ & $\begin{array}{l}\text { Weak - } 0 \\
\text { Moderate - } 8 \\
\text { Strong - } 3 \\
\text { Overall - Moderate }\end{array}$ \\
\hline
\end{tabular}


Appendix 11: Table 6: Summary of findings of studies included in care continuity review

\begin{tabular}{|c|c|c|c|c|c|c|c|c|}
\hline Article & $\begin{array}{l}\text { Study } \\
\text { Group } \\
\text { / Scale }\end{array}$ & Target Population & Sample Size & Data Collection & Data Analysis & $\begin{array}{l}\text { Findings / Domains / Components of Care } \\
\text { Continuity }\end{array}$ & - Study Limitations & $\begin{array}{l}\text { Quality } \\
\text { Appraisal } \\
\text { Rating (CASP } \\
\text { and COSMIN) }\end{array}$ \\
\hline $\begin{array}{l}\text { Rose et al. } \\
\text { (2009) }\end{array}$ & $\begin{array}{l}\text { CONTIN } \\
\text { U-UM }\end{array}$ & $\begin{array}{l}\text { Participants have a } \\
\text { diagnosis of psychosis } \\
\text { and have been in touch } \\
\text { with services for at } \\
\text { least } 2 \text { years. }\end{array}$ & $\begin{array}{l}\text { Phase 1: Focus } \\
\text { groups: } \\
n=32 ; \\
\text { Expert panels: } \\
n=10 ; \\
\text { Feasibility } \\
\text { study: } \\
n=37 \\
\text { Phase 2: N/A } \\
\text { Phase 3: } \\
n=176\end{array}$ & $\begin{array}{l}\text { Phase 1: Focus groups; two } \\
\text { expert panels; feasibility study. } \\
\text { Phase 2: Test-retest reliability } \\
\text { and validity. } \\
\text { Phase 3: Field trial. }\end{array}$ & $\begin{array}{l}\text { Phase 1: Not specified } \\
\text { Phase 2: Test-retest } \\
\text { reliability and validity } \\
\text { Phase 3: Not specified }\end{array}$ & $\begin{array}{l}\text { CONTINU-UM scale for measuring CoC emphasises the } \\
\text { service user perspective and produces the following } \\
\text { domains: } \\
\text { - } \text { Experienced continuity } \\
\text { - Accessing, choice and range of services } \\
\text { - Waiting for services } \\
\text { - Out of hours and crisis support } \\
\text { - Hospital discharge process } \\
\text { - Staff changes } \\
\text { - Informational CoC } \\
\text { - Individual progress } \\
\text { - Day centres } \\
\text { - Care planning } \\
\text { - Staff communication } \\
\text { - Peer support } \\
\text { - Avoiding services }\end{array}$ & $\begin{array}{l}\text { - CONTINU-UM developed and validated for a narrow } \\
\text { patient group, people with differential diagnoses not } \\
\text { included in study. } \\
\text { - Lack of input into the study from less well-engaged } \\
\text { service users } \\
\text { - Data analysis for Phase } 1 \text { and } 3 \text { not specified. } \\
\text { - Moderate rating given for methodological qualities of } \\
\text { psychometric validation according to COSMIN } \\
\text { checklist. }\end{array}$ & $\begin{array}{l}\text { Weak - } 0 \\
\text { Moderate - } 10 \\
\text { Strong }-3 \\
\text { Psychometric field - } \\
\text { Moderate } \\
\text { Overall - Moderate }\end{array}$ \\
\hline $\begin{array}{l}\text { Sweeney } \\
\text { et al. } \\
\text { (2012) }\end{array}$ & $\begin{array}{l}\text { CONTIN } \\
\text { U-UM }\end{array}$ & $\begin{array}{l}\text { Service users recruited } \\
\text { from seven CMHTs in } \\
\text { two South London NHS } \\
\text { Trusts with a diagnosis } \\
\text { of psychosis and in } \\
\text { contact with services } \\
\text { for at least two years, } \\
\text { on the CMHT caseload } \\
\text { for at least six months } \\
\text { and on enhanced care } \\
\text { programme approach. }\end{array}$ & $n=167$ & $\begin{array}{l}\text { Five focus groups. } \\
\text { CONTINU-UM. } \\
\text { Two expert panels of service } \\
\text { users. }\end{array}$ & $\begin{array}{l}\text { Thematic analysis used to } \\
\text { analyse data from focus } \\
\text { groups. } \\
\text { Domains with CONTINU-UM } \\
\text { explored through using } \\
\text { factor analysis. } \\
\text { Relationships between } \\
\text { CONTINU-UM scores and } \\
\text { health/social measures } \\
\text { explored through linear } \\
\text { regression and examination } \\
\text { of quartile results. }\end{array}$ & 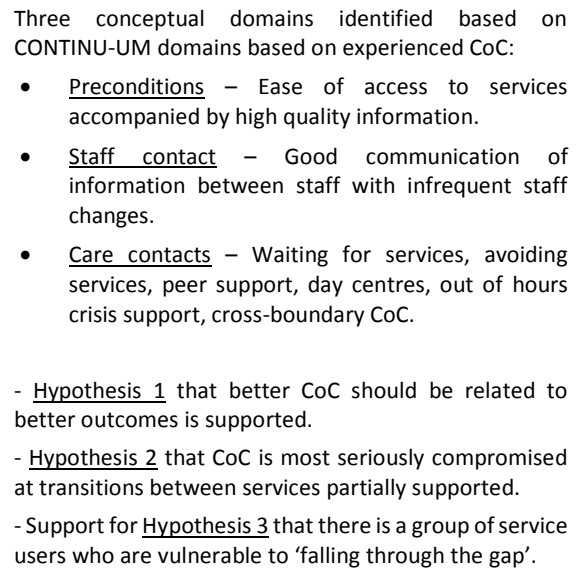 & $\begin{array}{l}\text { - Only } 36 \% \text { of eligible CNHT service users participated. } \\
\text { - Less well engaged service users not focused upon by } \\
\text { study data collection. } \\
\text { - Cross-sectional study may only be the basis for } \\
\text { inferring associations not causality. } \\
\text { - Outcomes chosen are not necessarily those } \\
\text { prioritised and valued by service users. }\end{array}$ & $\begin{array}{l}\text { Weak - } 1 \\
\text { Moderate - } 8 \\
\text { Strong - } 3 \\
\text { Overall - Moderate }\end{array}$ \\
\hline
\end{tabular}




\section{Appendix 11: Table 6: Summary of findings of studies included in care continuity review}

\begin{tabular}{|c|c|c|c|c|c|c|c|c|}
\hline Article & $\begin{array}{l}\text { Study } \\
\text { Group } \\
\text { / Scale }\end{array}$ & Target Population & Sample Size & Data Collection & Data Analysis & $\begin{array}{l}\text { Findings / Domains / Components of Care } \\
\text { Continuity }\end{array}$ & - Study Limitations & $\begin{array}{l}\text { Quality } \\
\text { Appraisal } \\
\text { Rating (CASP } \\
\text { and COSMIN) }\end{array}$ \\
\hline $\begin{array}{l}\text { Sweeney } \\
\text { et al. } \\
(2016)\end{array}$ & $\begin{array}{l}\text { CONTIN } \\
\text { U-UM }\end{array}$ & $\begin{array}{l}\text { - Service } \begin{array}{r}\text { users } \\
\text { diagnosed }\end{array} \text { with } \\
\text { psychosis recruited } \\
\text { from local CMHTs, } \\
\text { service user groups and } \\
\text { day centres. } \\
\text { - Professionals } \\
\text { recruited from 19 } \\
\text { CMHTs and associated } \\
\text { acute units. }\end{array}$ & $\begin{array}{l}\text { - Focus groups: } \\
n=32 \\
\text { - Professional } \\
\text { questionnaire } \\
\text { survey: } n=184\end{array}$ & $\begin{array}{l}\text { Five focus groups. } \\
\text { Professionals' questionnaire } \\
\text { survey. }\end{array}$ & $\begin{array}{l}\text { Thematic analysis used to } \\
\text { analyse data from focus } \\
\text { groups and also for question } \\
\text { asking to define Coc in } \\
\text { professionals' questionnaire } \\
\text { survey. } \\
\text { Conceptual mapping and } \\
\text { narrative comparison of } \\
\text { service user and professional } \\
\text { defined CoC models. }\end{array}$ & 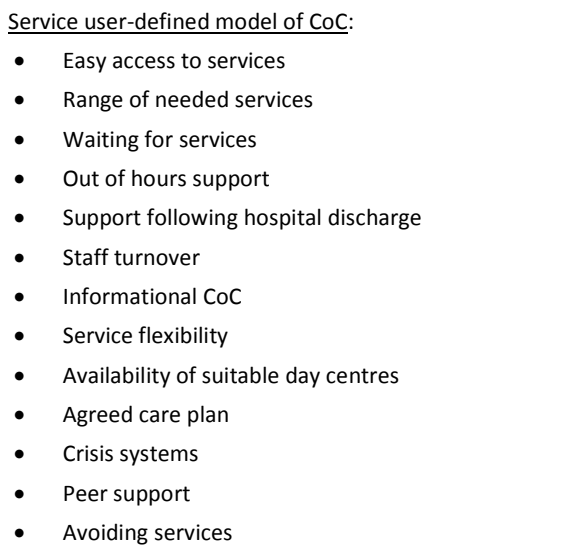 & $\begin{array}{l}\text { - Multiple methodologies used for qualitative analysis. } \\
\text { - Some bias and sampling issues present. }\end{array}$ & $\begin{array}{l}\text { Weak - } 0 \\
\text { Moderate - } 6 \\
\text { Strong - } 6 \\
\text { Overall - Strong }\end{array}$ \\
\hline $\begin{array}{l}\text { Adair et al. } \\
\text { (2003) }\end{array}$ & $\begin{array}{l}\text { ACSS- } \\
\mathrm{MH}\end{array}$ & $\begin{array}{l}\text { Articles from Medline, } \\
\text { Health-STAR, CINAHL } \\
\text { and PsycINFO. }\end{array}$ & $\begin{array}{l}\text { Literature } \\
\text { review } \\
n=305\end{array}$ & $\begin{array}{l}\text { Definitions and concepts } \\
\text { extracted by trained content } \\
\text { analysts. }\end{array}$ & Not specified & $\begin{array}{l}\text { - CoC concept considered crucial for the } \\
\text { management of people with serious mental illness } \\
\text { for more than } 40 \text { years. } \\
\text { - Consensus exists that COC is a multi-dimensional } \\
\text { concept and this should be reflected in } \\
\text { development of new measures with good } \\
\text { statistical and psychometric properties. } \\
\text { - Weak association found between CoC and } \\
\text { outcomes. }\end{array}$ & $\begin{array}{l}\text { - Analysis approach not specified. } \\
\text { - Quality appraisal for articles under review not } \\
\text { specified. }\end{array}$ & $\begin{array}{l}\text { Weak - } 1 \\
\text { Moderate - } 4 \\
\text { Strong - } 2 \\
\text { Overall - Moderate }\end{array}$ \\
\hline $\begin{array}{l}\text { Adair et al. } \\
\text { (2005) }\end{array}$ & $\begin{array}{l}\text { ACSS- } \\
\mathrm{MH}\end{array}$ & $\begin{array}{l}\text { Service users with a } \\
\text { confirmed diagnosis of } \\
\text { severe mental illness of } \\
\text { at least } 24 \text { months' } \\
\text { duration from } 70 \\
\text { directly funded } \\
\text { inpatient, outpatient, } \\
\text { emergency } \\
\text { departments and } \\
\text { CMHTs in three regions } \\
\text { in Alberta, Canada. }\end{array}$ & $\begin{array}{l}n=411 \\
\text { (endpoint } \\
\text { information } \\
\text { attained) }\end{array}$ & $\begin{array}{l}\text { CoC measured using the ACSS- } \\
\text { MH. Participants were } \\
\text { contacted by telephone at two } \\
\text { to three month intervals for } \\
\text { measurement of service use } \\
\text { events, housing services and } \\
\text { medications received. } \\
\text { Outcomes measured for } \\
\text { symptoms severity using the } \\
\text { Brief Psychiatric Rating Scale } \\
\text { (BPRS), the Multinomah } \\
\text { Community Ability Scale } \\
\text { (MCAS) for community } \\
\text { functioning, the Service } \\
\text { Satisfaction Scale-10, the } \\
\text { Wisconsin QoL Inventory } \\
\text { (WQLI) and the EQ-5D. }\end{array}$ & $\begin{array}{l}\text { Multi-linear regression } \\
\text { models for associations } \\
\text { between patient and } \\
\text { observer-rated continuity } \\
\text { and EQ-5D index scores. }\end{array}$ & $\begin{array}{l}\text { Factor structure is described as follows in a study using } \\
\text { the ACSS-MH scale: } \\
\text { - System fragmentation (perceived discontinuity } \\
\text { across services) } \\
\text { - Relationship base } \\
\text { - Responsiveness of treatment } \\
\text { Pattern of positive association between CoC and } \\
\text { outcomes - consistent association observed between } \\
\text { patient and observer rated CoC measure and health } \\
\text { outcomes over a } 17 \text { month period. }\end{array}$ & $\begin{array}{l}\text { - Confounding factors not clearly indicated. } \\
\text { - Small section on impact and further research. } \\
\text { - Minimal attrition } \\
\text { - Magnitude of difference in CoC scores related to } \\
\text { outcomes is not large. } \\
\text { - Bias may be present towards less seriously ill and } \\
\text { more compliant service users due to gatekeeper } \\
\text { influence. This may lead to an overly optimistic } \\
\text { appraisal of continuity. } \\
\text { - Moderate rating given for methodological qualities of } \\
\text { psychometric validation according to COSMIN } \\
\text { checklist. }\end{array}$ & $\begin{array}{l}\text { Weak - } 2 \\
\text { Moderate - } 6 \\
\text { Strong - } 4 \\
\text { Psychometric field - } \\
\text { Moderate } \\
\text { Overall - Weak }\end{array}$ \\
\hline
\end{tabular}




\section{Appendix 11: Table 6: Summary of findings of studies included in care continuity review}

\begin{tabular}{|c|c|c|c|c|c|c|c|c|}
\hline Article & $\begin{array}{l}\text { Study } \\
\text { Group } \\
\text { / Scale }\end{array}$ & Target Population & Sample Size & Data Collection & Data Analysis & $\begin{array}{l}\text { Findings / Domains / Components of Care } \\
\text { Continuity }\end{array}$ & Study Limitations & $\begin{array}{l}\text { Quality } \\
\text { Appraisal } \\
\text { Rating (CASP } \\
\text { and COSMIN) }\end{array}$ \\
\hline $\begin{array}{l}\text { Durbin et } \\
\text { al. (2004) }\end{array}$ & $\begin{array}{l}\text { ACSS- } \\
\mathrm{MH}\end{array}$ & $\begin{array}{l}\text { Sample drawn from } 77 \\
\text { programmes in } 3 \\
\text { regions in Alberta, } \\
\text { Canada. Individuals } \\
\text { with moderate to } \\
\text { severe mental illness. }\end{array}$ & $n=215$ & $\begin{array}{l}\text { - Service user survey using } \\
\text { ACSS-MH and collecting } \\
\text { additional information on } \\
\text { current service use and } \\
\text { perceived unmet need. } \\
\text { - Staff assessment package } \\
\text { using Colorado Client } \\
\text { Assessment Record (CCAR) } \\
\text { standardised measure of } \\
\text { client functioning and } \\
\text { service utilisation profile } \\
\text { for report of client's } \\
\text { current use and estimated } \\
\text { need. }\end{array}$ & 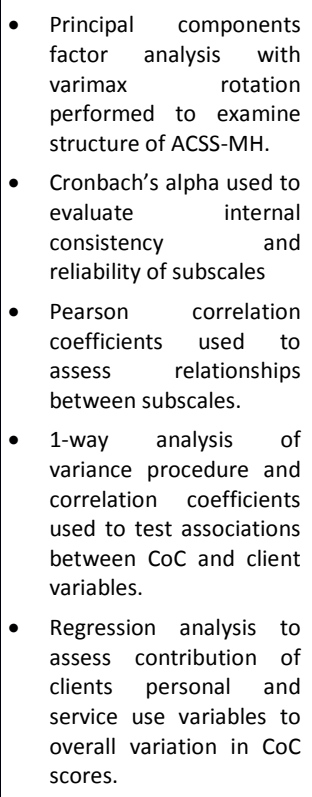 & $\begin{array}{l}\text { Three subscales of CoC identified within the ACSS-MH } \\
\text { CoC scale through psychometric analysis: } \\
\text { 1. System Access - user experience of the care system } \\
\text { and challenges involved with accessing the right } \\
\text { care. } \\
\text { 2. Interpersonal Aspects of Care-the extent to which } \\
\text { users perceive providers to be respectful, } \\
\text { collaborative and responsive. } \\
\text { 3. Care Team Function - well functioning team } \\
\text { delivering care in a timely and coherent way to the } \\
\text { client; shared care plan. } \\
\text { Informational continuity is excluded from this definition } \\
\text { of CoC on the basis that the client may not be in a } \\
\text { position to judge how well information transfer is } \\
\text { occuring between professionals. Consequently the } \\
\text { impact upon experienced continuity will be low. }\end{array}$ & $\begin{array}{l}\text { - Ethical approval not specified. } \\
\text { - Strong rating given for methodological qualities of } \\
\text { psychometric validation according to COSMIN } \\
\text { checklist. } \\
\text { - ACSS-MH performance across the severity continuum } \\
\text { is not assessed: only a small number of participants } \\
\text { have severe and complex conditions; clients with less } \\
\text { severe mental illness not included. }\end{array}$ & $\begin{array}{l}\text { Weak - } 1 \\
\text { Moderate - } 10 \\
\text { Strong - } 2 \\
\text { Psychometric field - } \\
\text { Strong } \\
\text { Overall - Moderate }\end{array}$ \\
\hline $\begin{array}{l}\text { Jensen et } \\
\text { al. (2014) }\end{array}$ & $\begin{array}{l}\text { ACSS- } \\
\mathrm{MH}\end{array}$ & $\begin{array}{l}\text { Participants receiving } \\
\text { treatment in } \\
\text { community psychiatric } \\
\text { centre and in a stable } \\
\text { condition in an urban } \\
\text { area in the vicinity of } \\
\text { Copenhagen. }\end{array}$ & $n=15$ & $\begin{array}{l}\text { Semi-structured interviews } \\
\text { focusing on illness narratives. }\end{array}$ & Content analysis & $\begin{array}{l}\text { The following domains of the CoC concept identified by } \\
\text { Joyce and associates (2004) are supported by qualitative } \\
\text { evidence: } \\
\text { - Accessibility of services } \\
\text { - Individualised care } \\
\text { - Relationship base } \\
\text { Flexibility and responsiveness of service delivery; } \\
\text { transfer of information between services. Discharge } \\
\text { planning. }\end{array}$ & $\begin{array}{l}\text { - Introductory literature review not based on most } \\
\text { recent literature } \\
\text { - Impact and further research not specified. } \\
\text { - Use of interpreters entails risk of miscommunication } \\
\text { or mistranslation. }\end{array}$ & $\begin{array}{l}\text { Weak - } 2 \\
\text { Moderate - } 4 \\
\text { Strong - } 3 \\
\text { Overall - Weak }\end{array}$ \\
\hline
\end{tabular}




\section{Appendix 11: Table 6: Summary of findings of studies included in care continuity review}

\begin{tabular}{|c|c|c|c|c|c|c|c|c|}
\hline Article & $\begin{array}{l}\text { Study } \\
\text { Group } \\
\text { / Scale }\end{array}$ & Target Population & Sample Size & Data Collection & Data Analysis & $\begin{array}{l}\text { Findings / Domains / Components of Care } \\
\text { Continuity }\end{array}$ & - Study Limitations & $\begin{array}{l}\text { Quality } \\
\text { Appraisal } \\
\text { Rating (CASP } \\
\text { and COSMIN) }\end{array}$ \\
\hline $\begin{array}{l}\text { Joyce et al. } \\
\text { (2004) }\end{array}$ & $\begin{array}{l}\text { ACSS- } \\
\mathrm{MH}\end{array}$ & $\begin{array}{l}\text { Articles from Medline, } \\
\text { PsycINFO, CINAHL and } \\
\text { HealthSTAR. } \\
\text { Participants with } \\
\text { severe and persistent } \\
\text { mental illness in the } \\
\text { Alberta region. }\end{array}$ & $n=36$ & $\begin{array}{lrr}\text { Literature } & \text { review } \\
\text { supplemented by } & \text { semi- } \\
\text { structured interviews } & \text { with } \\
\text { patients. } & & \end{array}$ & $\begin{array}{l}\text { Themes extracted from } \\
\text { literature examined by two } \\
\text { research teams; Team A and } \\
\text { Team B constructing a } \\
\text { hierarchical model and a list } \\
\text { of themes. These analyses } \\
\text { were then merged to create } \\
\text { a final domain model. }\end{array}$ & $\begin{array}{l}\text { Four domains identified within the ACSS-MH scale: } \\
\text { 1. Service delivery: Structural links, comprehensive } \\
\text { services, good information and communication } \\
\text { between services and providers, transition } \\
\text { management. } \\
\text { 2. Accessibility of services: referral timeline and } \\
\text { appointment location. } \\
\text { 3. Relationship base: relational / therapeutic } \\
\text { continuity. } \\
\text { 4. Individualised care: How well care is adapted to the } \\
\text { individual. }\end{array}$ & $\begin{array}{l}\text { - Quality appraisal for literature review part of study } \\
\text { not specified. } \\
\text { - Overreliance on consensus judgments between } \\
\text { teams may have led to the introduction of biases in } \\
\text { model formation. } \\
\text { - Sample size limited. }\end{array}$ & $\begin{array}{l}\text { Weak - } 1 \\
\text { Moderate - } 7 \\
\text { Strong }-4 \\
\text { Overall - Strong }\end{array}$ \\
\hline $\begin{array}{l}\text { Joyce et al. } \\
\text { (2010) }\end{array}$ & $\begin{array}{l}\text { ACSS- } \\
\mathrm{MH}\end{array}$ & $\begin{array}{l}\text { Adults with severe and } \\
\text { persistent mental } \\
\text { illness from } 70 \\
\text { inpatient, outpatient, } \\
\text { emergency and } \\
\text { community treatment } \\
\text { programmes in the } \\
\text { Alberta region. Both } \\
\text { rural and urban areas. }\end{array}$ & $\begin{array}{l}n=441 \\
\text { Subsample: } \\
n=171\end{array}$ & $\begin{array}{l}\text { - } \text { ACSS-MH scale } \\
\text { - } \text { Colorado Client } \\
\text { Assessment Record (CCAR) } \\
\text { - } \text { EQ-5D } \\
\text { - } \text { Wisconsin Quality of Life } \\
\text { Index } \\
\text { - } \text { MCAS } \\
\text { - } \text { BPRS } \\
\text { - Shortened version of the } \\
\text { Service Satisfaction Scale- } \\
30\end{array}$ & 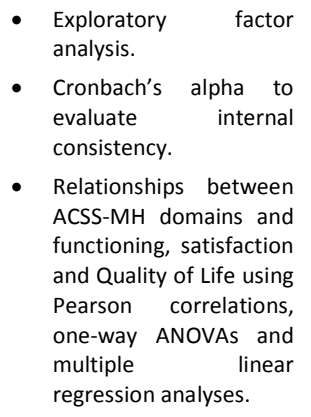 & $\begin{array}{l}\text { Three dimensions identified by factor analysis of ACSS- } \\
\text { MH scale: } \\
\text { 1. Individualised care - Perceived attentiveness to } \\
\text { individual needs, change in illness or life } \\
\text { circumstances. Quality of therapeutic relationship. } \\
\text { 2. Responsive system - Perception of a coherent } \\
\text { service system with good communication and } \\
\text { informational CoC. } \\
\text { 3. Responsive caregiver - Perceived flexibility and } \\
\text { responsiveness of primary provider. }\end{array}$ & $\begin{array}{l}\text { - Ethical approval not specified. } \\
\text { - Good distribution between urban and urban-rural } \\
\text { areas in Alberta. Representing } 70 \text { mental health } \\
\text { services. } \\
\text { - Multiple check and review stages. Blind techniques } \\
\text { used. } \\
\text { - Better functioning individuals may be under- } \\
\text { represented. } \\
\text { - ACSS-MH performance across the severity continuum } \\
\text { is not assessed. }\end{array}$ & $\begin{array}{l}\text { Weak - } 1 \\
\text { Moderate - } 5 \\
\text { Strong - } 7 \\
\text { Psychometric field - } \\
\text { Strong } \\
\text { Overall - Strong }\end{array}$ \\
\hline $\begin{array}{l}\text { Biringer et } \\
\text { al. (2017) }\end{array}$ & $\begin{array}{l}\text { Not } \\
\text { grouped }\end{array}$ & $\begin{array}{l}\text { Service users with SMI } \\
\text { at a community mental } \\
\text { health centre in } \\
\text { Norway. }\end{array}$ & $\begin{array}{l}n=10 \\
\text { participants } \\
\text { initially } \\
\text { interviewed. } \\
n=8 \\
\text { participants } \\
\text { interviewed at } \\
\text { follow-up } 27 \text { to } \\
30 \text { months } \\
\text { later. }\end{array}$ & $\begin{array}{l}\text { Semi-structured interviews } \\
\text { about experiences of } \\
\text { continuity of care across } \\
\text { services. } \\
n=8 \text { participants re- } \\
\text { interviewed two years later. }\end{array}$ & $\begin{array}{l}\text { Qualitative, hermeneutic- } \\
\text { phenomenological approach } \\
\text { to data analysis, which is } \\
\text { suited for in-depth } \\
\text { exploration of personal } \\
\text { experiences } \\
\text { understanding. }\end{array}$ & $\begin{array}{l}\text { Five themes representing experiences of continuity of } \\
\text { care were developed: } \\
\text { 1. } \\
\text { Relationship - Feeling safe in ongoing personal } \\
\text { relationships with minimal breaks in relationship. } \\
\text { 2. Timeliness - Getting help when needed and not } \\
\text { experiencing frustrating waiting times. } \\
\text { 3. Mutuality - Professionals and service users taking } \\
\text { initiatives together rather than a one-sided } \\
\text { struggle. } \\
\text { 4. Choice - Having an array of support options to } \\
\text { choose from. } \\
\text { 5. Knowledge - Feeling safe because one is informed } \\
\text { about what is going to happen rather than feeling } \\
\text { confused and insecure due to lack of knowledge. }\end{array}$ & $\begin{array}{l}\text { - Potential bias inherent in mental health expertise of } \\
\text { all researchers. } \\
\text { - Longitudinal aspect of study not fully determined. }\end{array}$ & $\begin{array}{l}\text { Weak }-1 \\
\text { Moderate }-2 \\
\text { Strong }-7 \\
\text { Overall - Strong }\end{array}$ \\
\hline
\end{tabular}




\section{Appendix 11: Table 6: Summary of findings of studies included in care continuity review}

\begin{tabular}{|c|c|c|c|c|c|c|c|c|}
\hline Article & $\begin{array}{l}\text { Study } \\
\text { Group } \\
\text { / Scale }\end{array}$ & Target Population & Sample Size & Data Collection & Data Analysis & $\begin{array}{l}\text { Findings / Domains / Components of Care } \\
\text { Continuity }\end{array}$ & Study Limitations & $\begin{array}{l}\text { Quality } \\
\text { Appraisal } \\
\text { Rating (CASP } \\
\text { and COSMIN) }\end{array}$ \\
\hline $\begin{array}{l}\text { Freeman } \\
\text { et al. } \\
\text { (2002) }\end{array}$ & $\begin{array}{l}\text { Not } \\
\text { grouped }\end{array}$ & $\begin{array}{l}\text { Field study participants } \\
\text { from home treatment } \\
\text { service for adults } \\
\text { suffering acute mental } \\
\text { health crisis, service for } \\
\text { women in mental } \\
\text { health crisis, and } \\
\text { Beacon site bridging } \\
\text { gap between primary } \\
\text { and secondary mental } \\
\text { health care. } \\
\text { Delphi study panel } \\
\text { were experts from } \\
\text { stakeholder groups. }\end{array}$ & $\begin{array}{l}\text { Literature } \\
\text { Review: } \\
n=91 \\
\text { Sample size not } \\
\text { provided for } \\
\text { field studies and } \\
\text { Delphi study }\end{array}$ & $\begin{array}{l}\text { Literature review: Medline, } \\
\text { Embase, PsycINFO, AHMED, } \\
\text { CINAHL, HMIC, HELMIS, Web of } \\
\text { Science, SIGLE. } \\
\text { Four field studies. } \\
\text { Delphi study - two } \\
\text { questionnaires. }\end{array}$ & $\begin{array}{l}\text { Literature review: five } \\
\text { questions on Coc. } \\
\text { Field studies involved } \\
\text { various case studies. } \\
\text { Delphis study aims to achieve } \\
\text { a consensus view on issues } \\
\text { from a group of experts. } \\
\text { Achieved by qualitative and } \\
\text { quantitative analysis of } \\
\text { panel answers to questions } \\
\text { on CoC from two } \\
\text { questionnaires. }\end{array}$ & 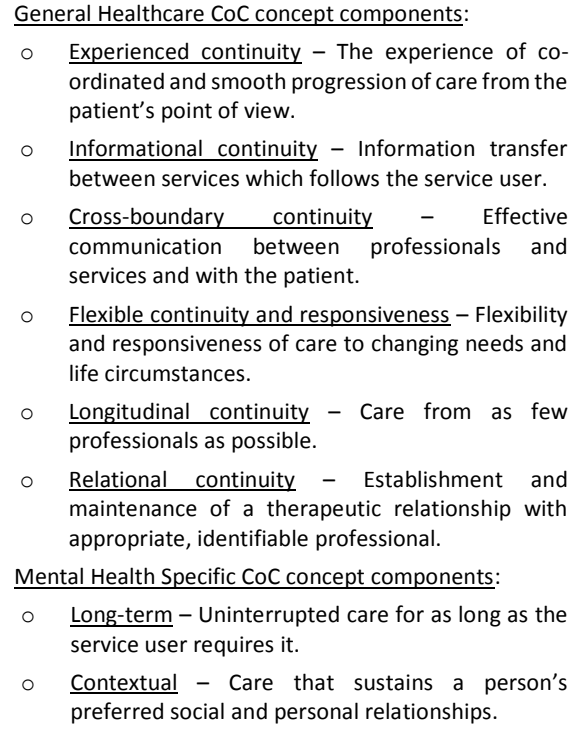 & $\begin{array}{l}\text { - Low response in Round } 2 \text { (using second } \\
\text { questionnaire) of Delphi study. } \\
\text { - Ethical approval not specified in this report. } \\
\text { - Quality appraisal not specified for literature review. }\end{array}$ & $\begin{array}{l}\text { Weak }-0 \\
\text { Moderate }-8 \\
\text { Strong }-5 \\
\text { Overall - Strong }\end{array}$ \\
\hline $\begin{array}{l}\text { Johnson et } \\
\text { al. (1997) }\end{array}$ & $\begin{array}{l}\text { Not } \\
\text { grouped }\end{array}$ & $\begin{array}{lr}\text { Literature } & \text { review } \\
\text { articles from } & \text { Medline } \\
\text { and PsychLit. }\end{array}$ & $\begin{array}{l}\text { Literature } \\
\text { review } \\
n=9\end{array}$ & Not specified. & Not specified. & $\begin{array}{l}\text { Two key dimensions of the CoC concept are identified - } \\
\text { Longitudinal CoC and Cross-boundary CoC. }\end{array}$ & $\begin{array}{l}\text { - Data extraction and analysis process not specified. } \\
\text { - Limited number of databases searched. } \\
\text { - Quality appraisal not specified. }\end{array}$ & $\begin{array}{l}\text { Weak }-1 \\
\text { Moderate }-6 \\
\text { Strong }-1 \\
\text { Overall - Moderate }\end{array}$ \\
\hline $\begin{array}{l}\text { Poremski } \\
\text { et al. } \\
\text { (2016) }\end{array}$ & $\begin{array}{l}\text { Not } \\
\text { grouped }\end{array}$ & $\begin{array}{l}\text { Service users and } \\
\text { service providers } \\
\text { involved in Coordinated } \\
\text { Access to Care from } \\
\text { Hospital Emergency } \\
\text { Departments (CATCH- } \\
\text { ED). }\end{array}$ & $n=13$ & Semi-structured interviews & $\begin{array}{l}\text { Thematic analysis restricted } \\
\text { to CoC dimensions given by } \\
\text { Haggerty and associates } \\
\text { (2003) and Bachrach (1981). }\end{array}$ & $\begin{array}{l}\text { Facilitators and barriers for experienced CoC concept } \\
\text { given by Haggerty and associates (2003) and Bachrach } \\
\text { (1981) confirmed by analysis: } \\
\text { Facilitators: } \\
\text { - Coordinated service navigation; facilitation of } \\
\text { services by care coordinator, especially community } \\
\text { based ones. } \\
\text { - Seamless transitions, for instance in discharge } \\
\text { planning. } \\
\text { - Therapeutic alliance. } \\
\text { Barriers: } \\
\text { - Difficulty engaging with services - either new or } \\
\text { sustained. } \\
\text { - Short service duration. }\end{array}$ & $\begin{array}{l}\text { - Study based on multidimensional CoC definition } \\
\text { given by Haggerty and associates (2003) and } \\
\text { Bachrach (1981). These are seminal though } \\
\text { potentially dated definitions of CoC within the } \\
\text { literature. } \\
\text { - No section on impact and further research. } \\
\text { - Quantitative outcomes and qualitative analysis not } \\
\text { matched. } \\
\text { - Thematic analysis was restricted to CoC dimensions } \\
\text { given by Haggerty and associates (2003) and } \\
\text { Bachrach (1981). }\end{array}$ & $\begin{array}{l}\text { Weak }-1 \\
\text { Moderate }-3 \\
\text { Strong }-5 \\
\text { Overall - Strong }\end{array}$ \\
\hline
\end{tabular}


Appendix 11: Table 6: Summary of findings of studies included in care continuity review

\begin{tabular}{|c|c|c|c|c|c|c|c|c|}
\hline Article & $\begin{array}{l}\text { Study } \\
\text { Group } \\
\text { / Scale }\end{array}$ & Target Population & Sample Size & Data Collection & Data Analysis & $\begin{array}{l}\text { Findings / Domains / Components of Care } \\
\text { Continuity }\end{array}$ & Study Limitations & $\begin{array}{l}\text { Quality } \\
\text { Appraisal } \\
\text { Rating (CASP } \\
\text { and COSMIN) }\end{array}$ \\
\hline $\begin{array}{l}\text { Puntis et } \\
\text { al. (2014) }\end{array}$ & $\begin{array}{l}\text { Not } \\
\text { grouped }\end{array}$ & $\begin{array}{l}\text { Literature review } \\
\text { articles from Medline } \\
\text { and PsychINFO. }\end{array}$ & $\begin{array}{l}\text { Literature } \\
\text { review } \\
n=18\end{array}$ & Not specified. & Not specified. & $\begin{array}{l}18 \text { studies show little consistency in the way the care } \\
\text { continuity concept is measured. } \\
\text { Little consistency in the way outcomes are measured. } \\
\text { Mixed association between COC and risk of } \\
\text { rehospitalisation. } \\
\text { Mixed association between COC and service satisfaction } \\
\text { - Adair and associates (2005) does find a relationship. } \\
\text { Consistency of evidence of association between } \\
\text { functioning and COC. } \\
\text { Review identifies emerging consensus about important } \\
\text { outcomes for measuring effects of COC in terms of } \\
\text { hospital readmission, symptom severity, social } \\
\text { functioning and service satisfaction. }\end{array}$ & $\begin{array}{l}\text { - Search strategy was fairly broad so it is possible } \\
\text { relevant articles were missed. } \\
\text { - Meta-analysis not possible due to different study } \\
\text { types under review. } \\
\text { - No interrater check at full-text eligibility stage. }\end{array}$ & $\begin{array}{l}\text { Weak }-0 \\
\text { Moderate }-6 \\
\text { Strong }-1 \\
\text { Overall - Moderate }\end{array}$ \\
\hline $\begin{array}{l}\text { Tomita and } \\
\text { Herman } \\
\text { (2015) }\end{array}$ & $\begin{array}{l}\text { Not } \\
\text { grouped }\end{array}$ & $\begin{array}{l}\text { Participants recruited } \\
\text { from transitional } \\
\text { residencies located on } \\
\text { the grounds of two } \\
\text { state-operated } \\
\text { psychiatric hospitals in } \\
\text { the New York City area. }\end{array}$ & $n=150$ & $\begin{array}{l}\text { Participants' status followed } \\
\text { over an } 18 \text { month period after } \\
\text { discharge. } \\
\text { Critical Time Intervention (CTI) } \\
\text { implemented for three process } \\
\text { outcomes: } \\
\text { - Perceived access to care } \\
\text { - Stability of patient-service } \\
\text { provider relationship } \\
\text { - Severity of instability of } \\
\text { patient-service provider } \\
\text { relationship }\end{array}$ & $\begin{array}{l}\text { Perceived access to care } \\
\text { analysed by comparison of } \\
\text { median group ratings at } \\
\text { nine-month and 18-month } \\
\text { follow-up. } \\
\text { Service provider stability } \\
\text { assessed using chi-square } \\
\text { test for comparison between } \\
\text { groups. } \\
\text { Severity of instability of } \\
\text { patient-service provider } \\
\text { relationship assessed } \\
\text { through non-parametric } \\
\text { quintile regression model } \\
\text { with bootstrap methods. } \\
\text { Correlation of 9-month Coc } \\
\text { measures raves with } \\
\text { homelessness and } \\
\text { psychiatric re-hospitalisation } \\
\text { outcomes. }\end{array}$ & $\begin{array}{l}\text { Assignment to CTI associated with more CoC over } \\
\text { several domains: CTI associated with higher perceived } \\
\text { access to care; lengths of working relationships with } \\
\text { psychiatrist and case manager significantly higher for } \\
\text { those assigned to CTI; quintile regression models } \\
\text { indicated those assigned to CTI had more favourable } \\
\text { physician transition rating. } \\
\text { Several nine-month CoC measures correlated with lower } \\
\text { risk of homelessness and re-hospitalisation. }\end{array}$ & $\begin{array}{l}\text { - Ethical approval not specified. } \\
\text { - Small sample size. } \\
\text { - Caregiver's perspective on CoC not included in study. }\end{array}$ & $\begin{array}{l}\text { Weak }-1 \\
\text { Moderate }-8 \\
\text { Strong }-2 \\
\text { Overall - Moderate }\end{array}$ \\
\hline
\end{tabular}




\section{Appendix 11: Table 6: Summary of findings of studies included in care continuity review}

\begin{tabular}{|c|c|c|c|c|c|c|c|c|}
\hline Article & $\begin{array}{l}\text { Study } \\
\text { Group } \\
\text { / Scale }\end{array}$ & Target Population & Sample Size & Data Collection & Data Analysis & $\begin{array}{l}\text { Findings / Domains / Components of Care } \\
\text { Continuity }\end{array}$ & Study Limitations & $\begin{array}{l}\text { Quality } \\
\text { Appraisal } \\
\text { Rating (CASP } \\
\text { and COSMIN) }\end{array}$ \\
\hline $\begin{array}{l}\text { Ware et al. } \\
\text { (2003) }\end{array}$ & $\begin{array}{l}\text { Not } \\
\text { grouped }\end{array}$ & $\begin{array}{l}\text { Adults diagnosed with } \\
\text { serious mental illness } \\
\text { using public mental } \\
\text { health services in and } \\
\text { around Boston, USA. }\end{array}$ & $\begin{array}{l}\text { Pilot test: } \\
n=41 \\
\text { Field test: } \\
n=400\end{array}$ & $\begin{array}{l}\text { Ethnographic data collected } \\
\text { through observation and } \\
\text { topically based open-ended } \\
\text { interviewing. } \\
\text { CONNECT was then } \\
\text { administered in the context of } \\
\text { cognitive interviews during } \\
\text { which there was examination } \\
\text { of meaning and relevance of } \\
\text { questionnaire items. }\end{array}$ & $\begin{array}{l}\text { Comprehensive } \\
\text { psychometric testing of } \\
\text { CONNECT through field test } \\
\text { for internal consistency, } \\
\text { scale reliability, convergent } \\
\text { reliability, test-retest } \\
\text { reliability and known-groups } \\
\text { validity. }\end{array}$ & $\begin{array}{l}\text { Five Domains for experienced continuity: } \\
\text { 1. Knowledge - practitioners' knowledge of their } \\
\text { clients. Quality of therapeutic relationship. } \\
\text { 2. Flexibility - creating flexibility - easy appointment } \\
\text { changes; practitioner goes out of his/her way to } \\
\text { help the user. } \\
\text { 3. Availability - practitioner availability. } \\
\text { 4. Care Coordination - practitioner coordination - is } \\
\text { mental health treatment well coordinated? } \\
\text { 5. Transitions - smoothing transitions: } \\
\text { o Discharge plan: Emergency services } \\
\text { transition, transition from one physician / } \\
\text { case manager/ therapist to another, housing } \\
\text { transition. } \\
\text { o Primary care - good contact and coordination } \\
\text { with primary care physician. } \\
\text { Two key dimensions identified - Longitudinal CoC and } \\
\text { Cross-sectional or Cross-boundary CoC. Good cross- } \\
\text { boundary CoC aids longitudinal CoC. } \\
\text { Weak relationship between CoC and health outcomes. }\end{array}$ & $\begin{array}{l}\text { - Ethical approval not specified. } \\
\text { - Sample bias may have occurred due to the sample } \\
\text { being volunteers. } \\
\text { - Field test only conducted in Boston area - May limit } \\
\text { generalisability of findings. } \\
\text { - There is room for improvements in psychometric } \\
\text { testing - a second round of psychometric testing } \\
\text { could incorporate additional measures of } \\
\text { psychological wellbeing. }\end{array}$ & $\begin{array}{l}\text { Weak }-1 \\
\text { Moderate }-10 \\
\text { Strong - } 2 \\
\text { Psychometric field - } \\
\text { Moderate } \\
\text { Overall - Moderate }\end{array}$ \\
\hline $\begin{array}{l}\text { Uijen et al. } \\
\text { (2014) }\end{array}$ & $\begin{array}{l}\text { Not } \\
\text { grouped }\end{array}$ & $\begin{array}{l}\text { Primary care patients at } \\
\text { risk for depression. } \\
\text { Patients with heart } \\
\text { failure. } \\
\text { Patients between } 18 \\
\text { and } 70 \text { years of age in } \\
23 \text { General Practices in } \\
\text { two regions in the } \\
\text { Netherlands. }\end{array}$ & $\begin{array}{l}n=264 \\
n=327\end{array}$ & $\begin{array}{l}\text { Patients' experienced } \\
\text { continuity of care measured } \\
\text { using a questionnaire including } \\
12 \text { items. }\end{array}$ & $\begin{array}{l}\text { Chi-square testing used to } \\
\text { compare the personal } \\
\text { continuity score between } \\
\text { the two patient groups. P- } \\
\text { value }<0.05 \\
\text { Multivariable analysis using } \\
\text { a general linear model to } \\
\text { compare total scores of } \\
\text { team and cross-boundary } \\
\text { continuity between the two } \\
\text { study groups. }\end{array}$ & $\begin{array}{l}\text { Conceptual components of care continuity: } \\
\text { - Personal continuity-number of care providers patients } \\
\text { contacted in General Practice. } \\
\text { - Team continuity in General Practice - the extent to } \\
\text { which care providers in General Practice had knowledge } \\
\text { of the patient and communicate / cooperate with each } \\
\text { other. } \\
\text { - Cross-boundary continuity - the extent to which GPs } \\
\text { and care providers outside the General Practice } \\
\text { communicate and cooperate with each other. } \\
\text { Most patients experienced a high level of collaboration } \\
\text { between care providers in General Practice, } 23 \% \\
\text { experienced a low level of collaboration between care } \\
\text { settings. } \\
\text { Patients at risk for depression experienced slightly } \\
\text { higher team continuity. }\end{array}$ & $\begin{array}{l}\text { - Impact and further research not specified. } \\
\text { - Depression and heart failure groups may not be } \\
\text { completely distinct. } \\
\text { - Demographic differences between two groups. } \\
\text { - Difference in recruitment strategy for two groups. } \\
\text { - Possible recall bias. }\end{array}$ & $\begin{array}{l}\text { Weak - } 1 \\
\text { Moderate - } 9 \\
\text { Strong - } 0 \\
\text { Overall - Moderate }\end{array}$ \\
\hline
\end{tabular}

